\title{
Electronic Poster Only
}

\author{
EP0-002 Adrenals and HPA Axis \\ Combined 17 alpha-hydroxylase/17, 20-lyase \\ deficiency in a Kuwaiti patient caused by a \\ homozygous point mutation in the CYP17A1 \\ gene \\ Hessa M Al Kandari'; Noriyuki Katsumata²; Sateesh K Kutty; \\ Alaa E Elshafey ${ }^{4}$ \\ ${ }^{1}$ Farwaniya Hospital, Pediatric, Farwania, Kuwait; ${ }^{2}$ National Research \\ Institute for Child Health and Development, Department of Molecular

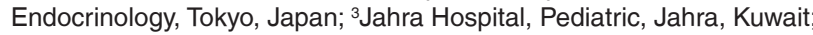 \\ ${ }^{4}$ Kuwait Medical Genetic Centre, Molecular genetic, Kuwait, Kuwait
}

Background: Combined 17 alpha-hydroxylase/17,20-lyase deficiency is a rare form of congenital adrenal hyperplasia characterized by hypokalemia, hypertension and sexual infantilism. It is considered a disorder of sexual development (DSD) which causes lack of virilization in 46, XY individuals. The disease is inherited in an autosomal recessive pattern and is caused by mutations in the gene encoding cytochrome P450c17 (CYP17A1).

Objective and hypotheses:We analyzed the CYP17A1 gene in a Kuwaiti girl with 17 alpha-hydroxylase/17, 20-lyase deficiency.

Methods: A 4-year-old (46, XY) female child born to consanguineous parents, presented with irregular heartbeats, hyper pigmentation, failure to thrive and developmental delay. Laboratory data showed hypokalemia, metabolic alkalosis with low plasma levels of cortisol, rennin and aldosterone and markedly elevated levels of 11-deoxycorticosterone and corticosterone. Serum levels of dehydroepiandrosterone (DHEA), androstenedione and testosterone were undetectable and did not show any response to ACTH stimulation. Results: Sequencing analysis of CYP17A1 gene revealed a homozygous mutation, p.A82D, changing codon 82 (GCC) encoding alanine to GAC encoding asparagic acid in the patient, while the parents were heterozygous for the same mutation.

Conclusions: This is the first report from Kuwait with a homozygous mutation in CYP17A1 causing combined loss of 17 alpha-hydroxylase/17, 20-lyase enzyme deficiency. Carmelo Salpietro

University of Messina, Department of Pediatrics, Messina, Italy

Background: It has been reported, in literature, that girls born small for gestational age (SGA) and obese girls present more frequently precocious adrenarche (PA) with respect to normal weight girls and those born appropriate for gestational age (AGA).

Objective: To compare, retrospectively, girls with isolated PA with respect to gestational age, body mass index (BMI), DHEAS, Delta4 androstenedione, insulin and basal 17OHP levels and after a standard ACTH test. Subjects and Methods: Forty-one patients (11SGA) with PA(chronological age $<8.0$ yrs) were evaluated for weight, height, bone age and BMI . Androgens and insulin concentrations were assessed for all children by commercial Kits. Statistical analysis was performed and data were expressed as median (minimum and maximum range).

Results: BMI-SDS (1.7 - range 0.1-2; and 1.5 -range 0.1- 2.5) and bone age/ chronological age ratio (1.3 vs 1.2) were superimposable in SGA and AGA girls. Respectively $27 \%$ of SGA and $30 \%$ of AGA were obese (BMI $>2$ SDS). Basal 17OHP serum levels (0.9-range $0.4-1.4$ vs 0.7 range $0.2-1.1 \mathrm{ng} / \mathrm{ml}$ ) and Delta $4(1.7$,range $0.8-2.6$ vs 1.6 , range $0.2-2.5 \mathrm{ng} / \mathrm{ml})$ were similar in both groups.

We found a statistical difference in basal DHEAS (292, range 189-410 vs 206.5 , range $76-426 \mu \mathrm{g} / \mathrm{dl} ; \mathrm{p}<0.005)$ and insulin levels $(5.7$, range 3.8-9.3 vs2.9, range 1.2-5.6 $\mu \mathrm{U} / \mathrm{ml} ; \mathrm{p}<0.001)$, and in stimulated 17OHP levels $(5.2$, range $2.4-7.8$ vs 3.9 , range $1.7-6,7 \mathrm{ng} / \mathrm{ml} ; \mathrm{p}<0.05$ ).

Conclusions: Overweight/obesity, present in our subjects, could be considered the most important factor for premature adrenarche. Insulin, DHEAS and ACTH stimulated 17OHP levels are significantly different in SGA vs AGA girls with isolated PA. Weight at birth and rapid infancy weight gain may be considered as risk factors for precocious increased levels of adrenal steroids in SGA girls.

\section{EPO-003 Adrenals and HPA Axis \\ Adrenocortical carcinoma as a cause of precocious puberty in one-year-old girl - case presentation \\ Hanna Borysewicz-Sanczyk ${ }^{1}$; Artur Bossowski'; Beata Sawicka'; Edyta Pietrewicz'; Janusz Pomaski'; Agnieszka Rudzka-Kocjan; \\ Elzbieta Moszczynska²; Maria Szarras-Czapnik² \\ ${ }^{1}$ Children's Teaching Hospital, Department of Paediatrics, \\ Endocrinology, Diabetology with the Cardiology Division, Bialystok, \\ Poland; ${ }^{2}$ Children's Memorial Health Institute, Department of \\ Endocrinology and Diabetology, Warsaw, Poland}

Background: Adrenal tumor is a rare disease characterizing by two morbidity peaks - smaller in children up to 10 years old and higher in adults between 40 and 50 years old. In about $50 \%$ of cases adrenal tumors are hormonally active presenting symptoms of Cushing's syndrome, virilization, feminization and occasionally hyperaldosteronism. In most cases tumor reaches considerable dimension at the moment of manifestation of first symptoms.

Objective and hypotheses: We present one-year and two-month-old girl which entered the Department of Paediatrics, Endocrinology, Diabetology with the Cardiology Division Medical University of Bialystok due to pubic hair.

Method: Additional symptom during physical examination was overgrown clitoris.

Results: Bone age agreed with metrical age. Hormonal analysis revealed 10 times higher concentration of testosterone $(784 \mathrm{mg} / \mathrm{ml})$ with normal DHEA-S concentration. Cortisol profile, ACTH and 17-OHP concentration were normal while alpha-fetoprotein concentration $(19,8 \mathrm{ng} / \mathrm{ml})$ and androstendion $(1795 \mathrm{ng} / \mathrm{ml})$ were elevated. Urine steroid profile revealed intense androgen's metabolites excretion characteristic for virilizing adrenal cortex tumor as well as slightly elevated of cortisol metabolites excretion, without hypercortisolemia. Ultrasonography of abdomen revealed hypoechogenic, round area of $25 \times 20 \mathrm{~mm}$ size in right adrenal. The area got stronger from 48 to $90 \mathrm{j}$. H. during second phase of computer tomography. Generative organ in ultrasonography was normal. In order to subsequent therapy the patient was directed to Children's Memorial Health Institute, Department of Endocrinology and 
Diabetology, Warsaw Poland and the right adrenal was surgically removed. Histopathological examination revealed adrenocortical carcinoma. The patient was qualified to antineoplastic therapy.

Conclusions: Adrenocortical carcinoma may be rare cause of precocious puberty.

\section{EPO-004 Adrenals and HPA Axis}

\section{Adrenocortical insufficiency in a child due to topical application of corticosteroids}

\section{Marina Krstevska-Konstantinova ${ }^{1}$; Nevenka Slaveska²;} Aleksandra Jancevska' ${ }^{1}$ Zoran Gucev ${ }^{1}$; Nina Caca ${ }^{3}$; Agron Starova $^{3}$ ${ }^{1}$ Pediatric Clinic, Endocrinology and genetics, Skopje, Macedonia, Fyrom; ${ }^{2}$ Pediatric Clinic, Metabolism, Skopje, Macedonia, Fyrom; ${ }^{3}$ Clinic of Dermatology, Dermatology, Skopje, Macedonia, Fyrom

Background: Application of topical steroids due to a dermatologic condition for a long time period suppresses the hypotalamic-pituitary-adrenal (HPA) axis. The patients may develop Cushing syndrome or adrenocortical insufficiency.

Objective and hypotheses: A four year old boy presented at our departements with simptoms of Cushing's disease and erythrodermia psoriatica. Previously he was treated for 1,5 years with glucocorticosteroid creams prescribed for his skin condition by his local dermatologist. His father has also psoriasis vulgaris. The boy's height and weight were at the 50 th percentile at the growth curve. His skin was thin, coarse, and with generalised erythema.

Methods: Serum AST, ALT, lipids, glycemia, morning cortisol and ACTH levels were measured. A low dose ACTH stimulation test was carreid out. Hydrocortisone was started for the prevention of glucocorticoid withdrawal syndrome and dose was gradually decreased.

Results: Cortisol levels were very low in this child below 1,0 ug/dl and after two weeks of treatment increased to $2,8 \mathrm{ug} / \mathrm{dl}$. It had normal values after one month. The ACTH stimulation test showed suppression of the H-P-A axis. His skin codition was treated by clinical dermatologists.

Conclusions: The side-effects of topical steroids are dangerous. Collaboration must exist between the physicians of different branches to avoid such coditions.

\section{EP0-005 Adrenals and HPA Axis}

\section{Triple A syndrome in 2 patients with ENT signs as first manifestations}

Isabelle Oliver Petit'; Marie Bournez' ; Eric Bieth'² Sophie Caula';

Maithé Tauber

${ }^{1}$ Hopital des enfants, Unité d'endocrinologie, Toulouse, France; ${ }^{2}$ Hopital

Purpan, Génétique médicale, Toulouse, France; ${ }^{3} \mathrm{H}$ opital des enfants,

Endocrinologie pédiatrique, Toulouse, France

Background: Triple A syndrome is a rare autosomal disorder characterized by adrenal insufficiency, alacrimia, achalasia, and impairment of nervous system. ENT manifestations have never been reported

Objective: To describe two patients with ENT signs as first manifestation referring to hospital

Cases report: 3 year old boy born from non-consanguineous Mediterranean parents was referred to our clinic for hypoglycemia and acute adrenal insufficiency. The patient and his 6 year old brother had a history of atypical chronic nasal and sinus scabby obstruction, with pyocyanic infection. Nor etiology nor efficient treatments were proposed. The identification of alacrimia and dysmorphic face raised the suspicion of triple A syndrome which was confirmed by the presence of homozygous c. $1331+1 \mathrm{G}>$ A mutation in AAAS gene in both children. Complementary investigations identified achalasia of the cardia and soft peripheral motor neuropathy in the 2 brothers and no mental retardation. Partial unilateral soft palate palsy was identified in the older patient but not in the younger one. Adrenal insufficiency was confirmed as well as in the older brother and glucocorticoid therapy was initiated for both. With gastric anti-secreting therapy and local nasal corticosteroid, nasal obstruction dramatically resolved.

Conclusion: These unusual ENT manifestations have not yet been reported in Triple A to our knowledge. We hypothesize that alacrimia with dry mucous membrane, achalasia with gastro esophageal reflux and soft palate dysfunction participated in its pathophysiology.

\section{EP0-006 Adrenals and HPA Axis \\ Cyp21A2 and Cyp11B1: first report of a digenic inheritance in CAH}

Soara Menabò; Angelica Marsigli; Lilia Baldazzi; Annalisa Nicoletti; Piero Pirazzoli; Antonio Balsamo

Azienda Ospedaliera S. Orsola-Malpighi; University of Bologna, Gynecology, Obstetric and Pediatrics, Bologna, Italy

Background: Congenital adrenal hyperplasia is caused mostly by 21 -hydroxylase deficiency $(>90 \%)$ and 11ß-hydroxylase deficiency $(5-8 \%)$. Both enzymes are required for cortisol synthesis and the non classical (NC) phenotype of both deficiencies is characterized by hyper-androgenic manifestations in childhood/adolescence.

Objective and hypotheses: To complete the characterization of a NC case in which the identified CYP21A2 mutations do not mach completely the phenotype by analysis of the CYP11B1 gene.

Methods: Case: female that present: precocious puberty at 5,5 yrs; at 6,7 yrs advanced bone age (10 yrs), hypertrophic clitoris and the following hormonal data: 17-OHP 8200/14600 ng/dl, ACTH 66 pg/ml; T $0.98 \mathrm{ng} / \mathrm{ml}$; Cortisol 190/170 ng/ml; D4-A 315/377 ng/dl. A therapy with hydrocortisone $(5 \mathrm{mg}$ $\mathrm{x} 3 /$ die) has been started. A first clinical diagnosis of NC-21OHD was made. Genetic analysis of CYP21A2 and CYP11B1 genes was performed as previously described.

Results: The sequencing analysis of CYP21A2 gene revealed the presence of 3 mutations: Q318X (null), A391T (mild) and *13 G>A 3'UTR (very mild) all carried by the maternal allele. Complete sequencing of the paternal CYP21A2 gene do not show any alterations. The MLPA analysis of the family revealed a normal arrangement of the locus, therefore all the 3 maternal mutations lie on a unique gene. In order to identify other possible molecular causes, we performed the CYP11B1 gene analysis even without a complete hormonal profile, as the patient was in therapy. This analysis revealed the presence of 2 mutations: R43Q and A386V, both present on the paternal allele and individually reported as mild.

Conclusions: The patient, with the standard clinical NC-CAH manifestations, resulted to be heterozygous for both the deficiencies. We suggest therefore the possibility of a cumulative effect on the phenotype of the two mutants as previously reported in other conditions for enzymes catalyzing consecutive reactions on the same pathway. This is the first report of a di-genic inheritance in $\mathrm{CAH}$.

\section{EPO-007 Adrenals and HPA Axis}

\section{Blepharophimosis-ptosis-epicanthus inversus syndrome and congenital adrenal hyperplasia in a 3 year-old girl}

Sophie Stoppa-Vaucher'; Manuela Decarli Diserens';

Marie-Claude Addor'; Franziska Phan-Hug'; Nelly Pitteloud' Michael Hauschild ${ }^{1}$

'University of Lausanne, Pediatric Endocrinology and Diabetology Unit, Lausanne, Switzerland; 2University of Lausanne, Genetics Unit,

Lausanne, Switzerland

Background: The Blepharophimosis-Ptosis-Epicanthus Inversus Syndrome (BPES) is a rare autosomal dominant disorder caused by mutations in the FOXL2 gene. The frequency of BPES is around 1 in 50,000. Type I includes the characteristic eyelid-malformations and premature ovarian failure, which is not found in type II. Congenital adrenal hyperplasia (CAH) due toCYP21 deficiency is one of the most common inborn conditions following an autosomal recessive inheritance. The classic form has a frequency of about 1 in 10,000 to 1 in 15,000, whereas the non-classic form is about 1 in 1,000 .

Objective: To provide the first description of concomitant BPES and CAH. Case report: A 3 year-old girl known to have BPES was referred because of a clitoromegaly. Family history revealed non-consanguineous Algerian parents whom father and paternal uncle were also known for BPES. Physical examination revealed an accelerated growth $(+10.4 \mathrm{~cm} / \mathrm{y}(+2.03 \mathrm{SD}))$ and typical signs for BPES. Clitoris measured $20 \times 4 \mathrm{~mm}$ without any other sign of virilization. Blood test found an increased morning $17-\mathrm{OH}-\mathrm{P}(59.8 \mathrm{nmol} / \mathrm{L}$; $\mathrm{N}<3$ ), and electrolytes were within the normal range as well as plasma renin activity. Adrenocorticotropin stimulation-test confirmed the diagnosis of nonclassic CAH (17-OH-P max $141 \mathrm{nmol} / \mathrm{L})$. Genetic testing demonstrated a missense heterozygous mutation (c.650C $>$ G, p.Ser217Cys) in the single exon of FOXL2 gene. Furthermore, an inherited heterozygous compound form with a genetic conversion in the exons 1,2 and 3 and a mutation V281L $(1685 \mathrm{G}>\mathrm{T})$ 
in exon 7 of the CYP21 gene was shown. Hydrocortisone was introduced leading to decreased growth velocity and stabilization of clitoris size.

Conclusions: To our knowledge, this is the first report describing the association of BPES and CAH in a young girl. Due to the high frequency of $\mathrm{CAH}$, this finding is probably incidental. Pubertal development must be followed closely.

\section{EP0-008 Adrenals and HPA Axis \\ Dynamic changes of adrenal axis in different kinds of growth hormone deficiency after growth hormone therapy}

Qian Wang; Guimei Li

Provincial Hospital Affiliated to Shandong University, Pediatric

Department, Jinan, China

Background: There was different influence in hypothalamus-pituitary-adrenal (HPA) axis in isolated growth hormone deficiency (IGHD) and multiple pituitary hormone deficiencies (MPHD). There has been some reports associated with significant changes in the HPA axis after rhGH therapy in adults with GHD. But related researchs about children have not been covered.

Objective and hypotheses: To investigate the dynamic changes of adrenal axis in growth hormone deficiency (GHD) children after recombinant human growth hormone (rhGH) therapy.

Methods: 50 patients were divided into Group IGHD and MPHD. These patients accepted rhGH injection and necessary hormone replacement, then followed up the levels of hormones in 3 monthly interval. Investigate the variance of adrenal axis between two groups.

Results: 60\% MPHD patients were defined as central hypoadrenalism before rhGH therapy, whose levels of cortison (COR) were under the normal range, and hydrocortisol replacement was necessary. There was a significant negative linear regression (hydrocortisone $(\mathrm{mg} / \mathrm{m} 2 / \mathrm{d}$ ) $=14.60-0.10 *$ preCOR (pmol/l)) between the therapy dosage of hydrocortisol and levels of COR before therapy. During rhGH therapy, there was steady decline of COR in Group MPHD, which had been out of normal range. Differing from group MPHD $(\mathrm{P}<0.01)$, there was a similar decrease of COR occurred in group IGHD, but mean levels were still maintained in the lower normal range. No severe adverse effects were discovered after hydrocortisol replacement.
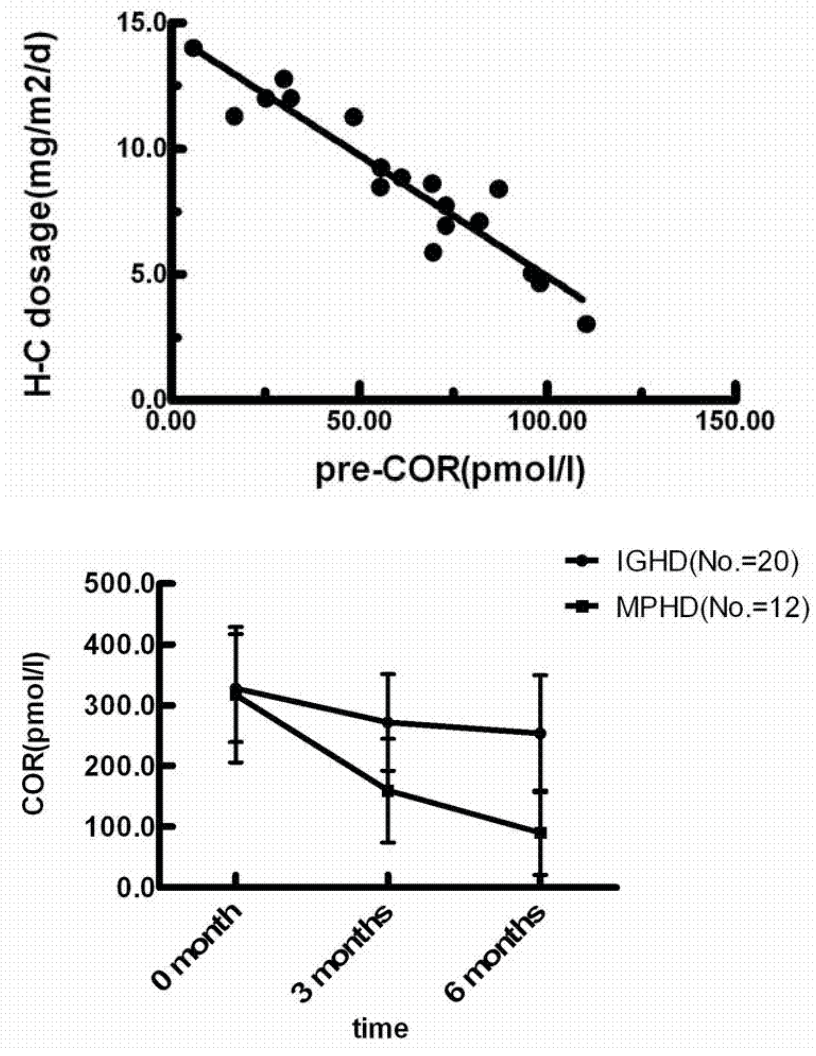

51st Annual Meeting of the ESPE
Conclusions: There are more obvious changes of adrenal axis observed in MPHD than in IGHD,that would be exacerbated during rhGH therapy. For safety and better effect of rhGH therapy, necessary hormone replacement and regular monitoring are necessary to maintain COR in lower limit.

\section{EPO-009 Adrenals and HPA Axis \\ Epidemiological, clinical, therapeutic and outcome patterns of congenital adrenal hyperplasia}

Thouraya Kamoun'; Mongia Hachicha'; Safa Ben Hadj Hamida ${ }^{\dagger}$ Adnène Hakim ${ }^{1}$; Sana Kmiha'; Lamia Sfaihi'; Abdellatif Nouri'; Hajer Aloulou'

${ }^{1} \mathrm{CHU}$ Hedi Chaker, Department of Pediatrics, Sfax, Tunisia; ${ }^{2} \mathrm{CHU}$ Fattouma Bourguiba, Department of Pediatrics Surgery, Monastir, Tunisia

Background: Congenital adrenal hyperplasia (CAH) refers to any of several autosomal recessive diseases resulting from mutations of genes for enzymes mediating the biochemical steps of production of cortisol from cholesterol by the adrenal glands. This is a diagnosis and therapeutic emergency that may cause the death of the patient.

Objective and hypotheses: The aim of this study was to determine the epidemiology, clinical features, treatement and outcomes of CAH.

Methods: This is a retrospective study of 26 cases of CAH diagnosed in our department between 1984 and 2011.

Results: There are 10 boys and 16 girls. Parental consanguinity was found in 21 cases. Similar cases in the family were found in 7 patients. An infant death in siblings was noted in 8 cases. The average age at diagnosis was 9.9 month. Two cases were diagnosed prenatally. Ambiguous genitalia was found in 16 cases. Vomiting with acute dehydration revealed the diagnosis in 4 cases. Precocious puberty was found in 5 cases. A salt wasting syndrome was found in 13 cases. The dosage of 17 hydoxyprogestérone plasma was elevated in 25 cases. Genotype was indentified in 7 cases . The are 21 cases of 21-hydroxylase deficiency, 3 cases of 11-beta hydroxylase deficiency and one case of 3 beta deshyrogénase deficiency. Medical treatment consisted of hydocortisone at a dose of $30 \mathrm{mg} / \mathrm{k} / \mathrm{m}^{2}$ day in 2or 3 doses in all patients, 9 alphafluorohydrocortisone was administered at a dose of 50 micrograms / day in 20 patients and $\mathrm{NaCl}$ in 13 patients. Genitoplasty feminizing was performed in 9 children.

Conclusions: $\mathrm{CAH}$ is a treatable disorder that occurs when the adrenal glands do not function properly. If left untreated, CAH will cause problems with growth and development and can cause life-threatening illness.

\section{EP0-010 Adrenals and HPA Axis}

\section{Adrenarche in children with Prader Willi} syndrome: effects of hydrocortisone treatment on bone maturation

Zeina Ajaltouni; Gwenaelle Diene; Catherine Molinas;

Emmanuelle Mimoun; Isabelle Kieffer; Jean-Pierre Salles Hopital des Enfants, Endocrinology, Toulouse, France

Background: PWS is a rare disease due to abnormalities in genomic imprinting of paternal chromosome 15 (15q11-13). Early diagnosis and multidisciplinary management in addition of growth hormone treatment is essential to these children and decrease morbidity and mortality. A high prevalence of precocious (before $8 \mathrm{yrs}$ in girls and 9 yrs in boys) adrenarche with pubarche (P2) and/or aggressive adrenarche is observed in $14-44 \%$ of these children. High levels of androgens and advanced bone age (BA) may compromise adult height albeit GH treatment.

Hypothesis: Bone maturation is mainly due to the peripheral production of oestrogen particularly from DHEA conversion. The decrease of DHEA by hydrocortisone treatment may be an alternative to block bone maturation in premature pubarche in these patients.

Methods: We present the following of 28 PWS children treated with hydrocortisone $(10 \mathrm{mg} / \mathrm{d})$ because of advanced BA. Among these 28 patients, $67 \%$ have $>6$ months BA advanced, $50 \%$ have $>1 \mathrm{yr}$ BA advanced and $25 \%$ have $>$ 2 yrs BA advanced.

Results: During following, BA advance remained stable and no dramatic maturation was reported. Median DHA level tend to remain stable.

Conclusion: Hydrocortisone treatment could prevent a dramatic bone matu- 
ration and is well tolerated in term of weight gain, effect on glycemic balance. More studies are necessary to conclude on a significant effect of this therapy.

\begin{tabular}{|c|c|c|c|c|c|}
\hline & P2 $N=28$ & $\begin{array}{l}\underset{N=28}{B} \\
\text { Beginning } H C\end{array}$ & $\begin{array}{c}1 \mathrm{yr} \mathrm{HC} \\
\mathrm{N}=23\end{array}$ & $\begin{array}{c}2 \text { yrs HC } \\
N=15\end{array}$ & $\begin{array}{c}\text { Last visit } \\
\text { HC>2yrs N=15 }\end{array}$ \\
\hline Age & $\begin{array}{c}7.9 \\
{[6.4-8.5]}\end{array}$ & $\begin{array}{c}8.4 \\
{[7.5-9.8]}\end{array}$ & $\begin{array}{c}9.4 \\
{[8.5-10.7]}\end{array}$ & $\begin{array}{c}10.5 \\
{[9.8-12.6]}\end{array}$ & $\begin{array}{c}12.5 \\
{[11.5-16.8]}\end{array}$ \\
\hline Weight (SD) & $\begin{array}{c}1.0 \\
{[0.2-2.5]}\end{array}$ & $\begin{array}{c}1.2 \\
{[0.1-2.4]}\end{array}$ & $\begin{array}{c}1.4 \\
{[0.3-2.5]}\end{array}$ & $\begin{array}{c}0.8 \\
{[-0.2-2.4]}\end{array}$ & $\begin{array}{c}1.2 \\
{[0.4-2.4]}\end{array}$ \\
\hline Height (SD) & $\begin{array}{c}-0.2 \\
{[-1.2-0.6]}\end{array}$ & $\begin{array}{c}0 \\
{[-0.6-1.3]}\end{array}$ & $\begin{array}{c}-0.3 \\
{[-0.7-0.5]}\end{array}$ & $\begin{array}{c}-0.3 \\
{[-1.2-0.6]}\end{array}$ & $\begin{array}{c}-0.8 \\
{[-1.6-0.3]}\end{array}$ \\
\hline BMI (Zscore) & $\begin{array}{c}1.8 \\
{[0.9-2.6]}\end{array}$ & $\begin{array}{c}1.6 \\
{[0.8-2.5]}\end{array}$ & $\begin{array}{c}1.9 \\
{[0.8-2.6]}\end{array}$ & $\begin{array}{c}2 \\
{[0.7-2.8]}\end{array}$ & $\stackrel{2}{2}[1.3-3.1]$ \\
\hline Waist/hip ratio & $\begin{array}{c}0.86 \\
{[0.84-0.88]}\end{array}$ & $\begin{array}{c}0.85 \\
{[0.83-0.89]}\end{array}$ & $\begin{array}{c}0.85 \\
{[0.82-0.90]}\end{array}$ & $\begin{array}{c}0.84 \\
{[0.82-0.92]}\end{array}$ & $\begin{array}{c}0.83 \\
{[0.79-0.88]}\end{array}$ \\
\hline Advance BA (yr) & $\begin{array}{c}0.9 \\
{[-0.2-1.8]} \\
n=25\end{array}$ & $\begin{array}{c}1.6 \\
{[0.8-2.2]} \\
n=25\end{array}$ & $\begin{array}{c}1.1 \\
{[0.5-1.7]} \\
n=15\end{array}$ & $\begin{array}{c}0.4 \\
{[-0.1-1.8]} \\
n=8\end{array}$ & $\begin{array}{c}1.2 \\
{[0.4-1.3] n=7}\end{array}$ \\
\hline $\mathrm{GH}$ treatment & $82.1 \%$ & $96.4 \%$ & $100 \%$ & $100 \%$ & $100 \%$ \\
\hline $\begin{array}{l}\text { GH dose } \\
\text { (mg/kg/week) }\end{array}$ & $\begin{array}{c}0.19 \\
{[0.12-0.21]}\end{array}$ & $\begin{array}{c}0.16 \\
{[0.12-0.21]}\end{array}$ & $\begin{array}{c}0.17 \\
{[0.14-0.20]}\end{array}$ & $\begin{array}{c}0.16 \\
{[0.15-0.22]}\end{array}$ & $\begin{array}{c}0.14 \\
{[0.13-0.21]}\end{array}$ \\
\hline $\begin{array}{l}\text { HC dose } \\
(\mathrm{mg} / \mathrm{m} 2)\end{array}$ & & $\begin{array}{c}9.5 \\
{[8.2-10.5]}\end{array}$ & $\begin{array}{c}8.9 \\
{[7.5-9.6]}\end{array}$ & $\begin{array}{c}8.3 \\
{[7.1-8.7]}\end{array}$ & $\begin{array}{c}7 \\
{[6-7.5]}\end{array}$ \\
\hline \multicolumn{2}{|l|}{$\mathrm{HC}$ duration } & & & & $4.2[3.7-4.5]$ \\
\hline
\end{tabular}

\section{EP0-011 Adrenals and HPA Axis}

\section{Is continuous hydrocortisone infusion in a 12 year old boy with Addison's disease more effective than an oral dosing regimen? \\ Abigail Atterbury; Carly Redington; Peter Hindmarsh \\ Great Ormond Street Hospital, Endocrinology, London, United \\ Kingdom}

Background: Addison's disease is an extremely rare disorder of the adrenal glands, with a 14:100,000 percentage of in the UK population. Many patients with primary adrenal insufficiency experience symptoms such as fatigue and poor quality of life in spite of conventional replacement therapy with glucocorticoids and mineralocorticoids (1). It has been suggested in poorly responding adult patients with Addison's disease that continuous hydrocortisone infusion monitoring could be a viable treatment option.

Objective and hypotheses: This poster will present the case of a 12 year old boy with Addison's disease. Initially treated with oral replacement doses, it will discuss his symptoms on this regimen, before discussing the practicalities of initiating pump therapy. Quality of life symptoms as well as cortisol profiles pre and post regime will be detailed.

Results: In this section cortisol levels will be shown pre and post pump regime in both table and graph format. A comparison of symptoms and quality of life issues will also be discussed although this has not been performed using a formal scoring system.

Conclusions: In the case of this 12 year old boy, since commencing his continuous infusion there have been significant improvements in his quality of life. He has returned to full time education and is fully independent in his pump regime, including emergency management. The circadian cortisol rhythms demonstrated in the graphs also show a much more controlled treatment than previously seen, with a lower dose of corticosteroids therefore eliminating some side effects previously seen.

\section{EP0-012 Adrenals and HPA Axis}

\section{Type II aldosterone synthase deficiency (18-hydroxycorticosterone dehydrogenase deficiency) as a cause of failure to thrive}

Carsten Döing'; Gitta Kameda ${ }^{1}$; Stefan Wudy²; Michaela Hartmann²; Sebastian Kummer'; Ertan Mayatepek ${ }^{1}$; Thomas Meissner ${ }^{1}$

${ }^{1}$ Heinrich-Heine-University, Department of General Paediatrics, Düsseldorf, Germany; 2Justus-Liebig-University, Department of General Paediatrics, Giessen, Germany

Background: Defects in mineralocorticoid synthesis are rare causes for congenital adrenal hyperplasia. Manifestations range from mild failure to thrive during childhood to severe neonatal salt wasting crisis..Type II aldosterone synthase deficiency is caused by mutations in the CYP11B2 gene and are characterized by an elevated 18-hydroxycorticosterone concentration and a decreased plasma aldosterone level.

Objective and hypotheses: Despite its rareness, different defects in mineralocorticoid synthesis should be considered as differential diagnosis also in neonates and infants with common symptoms like failure to thrive.

Patient: A male infant with a birth weight of $2950 \mathrm{~g}$ failed to gain weight. He presented acutely to hospital at age 6 months due to an gastroenteritis. His weight was $4,19 \mathrm{~kg}(1,6 \mathrm{~kg}<3$. percentile $)$ and length was $60,5 \mathrm{~cm}(3,5 \mathrm{~cm}$ $<3$.percentile). The newborn screening for inborn errors of metabolism was normal. The parents were unrelated of Turkish origin with an unremarkable family history.

Results: The patient showed persistent hyponatremia along with hyperkalemia. Levels of 17-hydroxyprogesterone and adrenocorticotropic hormone were in the normal range. However, elevated plasma aldosterone in association with highly increased renin levels were suggestive of a defect in mineralocortosteroid synthesis. Chromatographic examination of spontaneouslyvoided urine revealed elevated 18-hydroxycorticosterone levels indicating a 18-hydroxysteroid-dehydrogenase deficiency. Other abnormalities in the glucocorticoid synthesis were not found. Genetic analysis confirmed the 18-hydroxysteroid-dehydrogenase deficiency. Treatment was initiated with $20 \mu \mathrm{g} / \mathrm{kg}$ of fludrocortisone daily. Laboratory parameters normalized within two weeks and the patient showed rapid catch-up growth with weight reaching the 3rd percentile at the age of 11 months.

Conclusions: In patients with failure to thrive and persistent abnormal serum electrolyte levels, a defect in mineralocorticoid synthesis should be considered.

\section{EPO-013 Adrenals and HPA Axis \\ Isolated glucocorticoid deficiency with transient mineralocorticoid deficiency and severe developmental delay}

Abdelhadi M Habeb ${ }^{1}$; Claire R Hughes ${ }^{2}$; Abdallah Al-Harbi'; Amal Al-Johani'; Louise A Metherel/2

${ }^{1}$ Maternity and Children Hospital, Paediatrics, Al-Madinah, Saudi Arabia; ${ }^{2} \mathrm{WHRI}$, Barts and the London school of Medicine, QMUL, Endocrinology, London, United Kingdom

Background: Adrenal insufficiency and neurological features are associated with Triple A syndrome (TAS) and adrenoleukodystrophy (ALD). However neurological sequelae following severe hypoglycaemia secondary to glucocorticoid deficiency (GD) is well described. Intermittent mineralocorticoid deficiency (MD) has been reported with mild StAR mutations or severe loss of function MC2R mutations.

Objective: We report a child with primary adrenal failure and neurological features in whom the final diagnosis is not clear.

Methods: Investigations including appropriate gene sequencing were carried out in an attempt to ascertain the cause of adrenal insufficiency.

Results: A term girl of first degree cousins presented with an adrenal crisis at 18 days. She had hyperpigmentation, nystagmous, microcephaly and normal external female genitalia. Initial investigations revealed $\mathrm{Na} 118 \mathrm{mmol} / \mathrm{l}, \mathrm{K}$ $8.2 \mathrm{mmol} / 1$, glucose $2.7 \mathrm{mmol} / 1$, cortisol $37 \mathrm{nmol} / 1$, ACTH $796 \mathrm{pg} / \mathrm{ml}$ and normal 17-OHP. She improved rapidly with IV fluids and hydrocortisone and was commenced on hydrocortisone and fludrocortisone. Ultrasonagraphy showed normal adrenals and female internal organs with karyotype 46XX. Further evaluation excluded TAS (normal tear production and barium swallow) and ALD (VLCFA normal). MRI showed brain atrophy including optic atrophy. She failed to attend follow-up until aged 14 months. On review she had been unexpectedly off fludrocortisone for the previous 3 months yet had normal electrolytes and renin. MC2R, MRAP and StAR gene sequencing were normal. At 5 yrs she had developmental delay but her electrolytes remained normal on hydrocortisone replacement alone.

Conclusions: This child has isolated GD with transient mild MD that resolved with hydrocortisone treatment without mutation in the known genes responsible for FGD. Her neurological deficit may be secondary to neonatal hypoglycaemia however the microcephaly and nystagmous at birth may indicate a novel association between adrenal insufficiency and neurological disease. 


\section{EPO-014 Adrenals and HPA Axis}

False positive dexamethasone suppression test in a patient treated with carbamazepine

Filiz Tutunculer; Digdem Bezen; Emine Dilek; Asli Durmus

Medical School Of Trakya University, Pediatric Endocrinology, Edirne, Turkey

Introduction: The overnight 1-mg dexamethasone suppression test (DST) is widely used as a screening test for Cushing's syndrome. The test can be falsely positive in several conditions, that affect either cortisol secretion or the metabolism of dexamethasone. Dexamethasone is primarily metabolized by hepatic CYP3A4, an enzyme complex that is responsible for the metabolism of many xenobiotics. It is known that antiepileptic drugs such as diphenylhydantoin, phenobarbital, carbamazepine result in false positive DST.

Case: A 9 years old boy was referred to the clinic for investigation of his progressive weight gain in recent years. He had known history of epilepsy, for which he has been taken carbamazepine. He had no history of steroid use. Physical examination revealed weight of $57.4 \mathrm{~kg}(>97 \mathrm{p},+4.1 \mathrm{SD})$, height of $137,7 \mathrm{~cm}(75 \mathrm{p},+0,8 \mathrm{SD})$, blood pressure $100 / 70 \mathrm{mmHg}$, cushingoid features with facial plethore. Tanner stage was G1P1. Laboratory findings showed that serum electrolytes, lipid profile and oral glucose tolerance test were normal. Because he had clinical signs suggestive of Cushing's syndrome, twice he underwent a 1-mg overnight DST, and each time failed to suppress his morning cortisol level (10,3 mcg/dl and 20,5 mcg/dl recpectively). Baseline adrenocorticotrophic hormone was normal $(24.1 \mathrm{pg} / \mathrm{ml})$. Further investigations showed normal daily variation in cortisol level, with a low midnight cortisol level of $1,6 \mathrm{mcg} / \mathrm{dl}$ and $18.5 \mathrm{mcg} / \mathrm{dl}$ in the morning. His $24-\mathrm{h}$ urinary free cortisol level was within the normal range $(31.3 \mathrm{nmol} / \mathrm{day})$. We suspected that the DST was falsely positive because of induction of CYP3A4 by carbamazepine. Conclusions: In screening Cushing's syndrome, be aware of potential drug interaction with dexamethasone, which may lead to false positive results.

\section{EPO-015 Adrenals and HPA Axis}

\section{Evaluation of patients with premature pubarche}

Emine Dilek; Digdem Bezen; Filiz Tutunculer

Medical School of Trakya University, Pediatric Endocrinology, Edirne, Turkey

Background: Premature pubarche (PP) is the main manifestation of premature adrenarche. Differential diagnosis and follow up of the patients with PP should be done.

Objective: To evaluate the clinical characteristics and laboratory findings of the patients with PP who consulted to the Pediatric Endocrinology from January 2007 to January 2012.

Methods: One hundred fifty four patients were evaluated for weight, height, Tanner stage, body mass index (BMI) Z score, bone age and serum androgen levels.

Results: We analyzed 144 girls $(93,5 \%)$ and 10 boys $(6,5 \%)$. The mean ages at first visit were 7,6 $\pm 1,1$ (range 4,9-10) years in girls and 8,3 $\pm 0,6$ (range 6,99,4 ) years in boys, mean weight was $29,1 \pm 5,9 \mathrm{~kg}$ and $33,4 \pm 6,4 \mathrm{~kg}$, and mean height was $126,6 \pm 11 \mathrm{~cm}$ and $133,6 \pm 5,8 \mathrm{~cm}$, respectively. BMI Z score of the girls was $0,63 \pm 1,21$ and of the boys was $0,89 \pm 0,91 ; 25,3 \%$ of the patients were overweight (36 girls, 3 boys). Fifty patients (32,5\%) had advanced bone age, $100(64,9 \%)$ had bone age compatible with chronologic age and 4 (2,6\%) had delayed bone age. The pubic hair was the most common presenting complaint $(91,5 \%)$ and was followed by axillary hair $(33,7 \%)$ and axillary odor $(4,5 \%)$. The mean time between the onset of symptoms and the admission to hospital was 9,2 $\pm 8,5$ months (range 1-60). On physical examination none of the girls had clitoromegaly. No patient was due to tumor nor exogenous androgen. DHEAS concentrations was $86,6 \pm 60,5 \mathrm{mcg} / \mathrm{dl}$. Standart dose ACTH test was performed in 51 patients and $20(39,2 \%)$ of them had 17-hydroxyprogesterone levels up to $3 \mathrm{ng} / \mathrm{ml}$.

Conclusions: PP was more frequent and earlier aged onset in girls than boys. Overweight could be considered an important factor for PP.

\section{EP0-016 Adrenals and HPA Axis \\ Precociuos pubarche in a girl with a 48,XXXX karyotype}

Grazia Morandi; Evelina Maines; Giulia Rodella; Francesco Doro; Paolo Cavarzere; Elena Monti; Rossella Gaudino; Franco Antoniazzi University of Verona, Department of Life and Reproduction, Verona, Italy

Introduction: Only around 40 cases of $48, \mathrm{XXXX}$ have been reported in the literature so far. Their phenotypes are very heterogeneous, but the number of $\mathrm{X}$ chromosome seems to be fundamental for normal growth, ovarian function and mental development.

Case report: A 8,4 years old girl with a 48,XXXX karyotype came to our Clinic for pubic hair appeared 8 months before. She had an height $>3 \mathrm{SD}$, a weight $>3 \mathrm{SD}$, a prominent frontal eminences, exophthalm, low-set ears and a flat nasal bridge with a shield shaped thorax, joint laxity and valgism of the knee and a mental delay. Her bone age (BA) advanced fastly: from 8 to 9 years and 9 months after less than a year. ACTH test resulted negative, the Gonadotrophin-releasing hormone analogue (GnRHa) test showed basal FSH and LH levels of 13,3 and 1,3 U/1 respectively and stimulated FSH and LH levels of 91,6 and 74,4 U/1. Estradiol was 21,9 pg/L. At the pelvic ultrasound the uterus longitudinal diameter was of $33 \mathrm{~mm}$. RMN was not performed because the patient's parents refusal. For the pubertal response to GnRHa test, the BA acceleration and the mental retardation, we treated her as a central precocious puberty (CPP) with the complete arrest of puberty progression. GnRHa treatment was suspended at 12 years of BA and after 6 we performed a new GnRHa test, which revealed a clear picture of hypergonadotropic hypogonadism (LH 14,8 U/L before and 173,0 U/L after stimulus; FSH 40,7 U/L before and 110,0 U/L after stimulus, with levels of estradiol < 0,20 pg/ml). Conclusion: Patients with more or less X chromosome require a right followup as they can show gonadal dysgenesis, with just a FSH increased alone, later than Turner syndrome. An unsolved question remains: "Have the pubarche and the precocious activation of hypothalamic-pituitary axis initially hidden the hypergonadotropic hypogonadism?"

\section{EPO-017 Adrenals and HPA Axis}

\section{Case report: adrenal insufficiency due to glycerol kinase deficiency}

Cristiane Kopacek' ${ }^{1}$ Carolina Fishinger Souza ${ }^{2}$; Raquel Borges Pinto ${ }^{3}$; Anelise Uhlmann ${ }^{4}$

${ }^{1}$ Hospital da Criança Santo Antonio, Pediatric Endocrinoloy, Porto Alegre, Brazil; ${ }^{2}$ Hospital de Clínicas de Porto Alegre, Genetic, Porto Alegre, Brazil; ${ }^{3} \mathrm{Hospital}$ da Criança Conceição, Pediatric Gastroenterology, Porto Alegre, Brazil; ${ }^{4}$ Hospital da Criança Conceição, Pediatric Nephrology, Porto Alegre, Brazil

Background: Adrenal insufficiency ist most frequently related to congenital adrenal hyperplasia $(\mathrm{CAH})$ in the newborn. However, other conditions may cause primary adrenal insufficiency, rarely including congenital adrenal hypoplasia.

Objective and hypotheses: To report a rare condition associated to adrenal insufficiency in the newborn with adrenal crisis and further diagnosis of glicerol kynase deficiency (GKD), an X-linked recessive disorder.

Methods: A boy, borned with $3150 \mathrm{~g}$ and $50 \mathrm{~cm}$, APGAR score 4/8, first child of non-consanguineous parents. With $40 \mathrm{~h}$ of life, presented hypoglicemia (hemoglucotest $=10$ ) and received endovenous glucose. With 15 days of life was admitted at a emergency care unit, with severe dehydration an hyponatremia. and diagosis of adrenal insufficiency was considered. $17 \mathrm{OH}$ progesteron and androgen levels were normal, excluding CAH. Glucocorticosteroid and mineralocorticosteroid was iniciated with clinical recovering. With 1 year 9 months, due to severe failure to thrive, hypotonia and impaired neurologic development, most investigation was made.

Results: Laboratory test showed AST: 250/295 U/L, ALT: 215/289 U/L. Cholesterol $175 \mathrm{mg} / \mathrm{dl}$ e Triglicerydes: $568 \mathrm{mg} / \mathrm{dl}$. Autoimmune test and biochemical markers of infectious diseases and hepatitis were normal; normal thyroid function, but low levels of IGF-1 (37, for normal levels $>51$ ) and indetectable levels of $25 \mathrm{OH}$ vitamin D (proper treatment was iniciated); ACTH 16,9 (with $0,23 \mathrm{mg} / \mathrm{m} 2$ of Dexamethasone and renin of 0,5 with $1 \mathrm{mg}$ of fludrocortisone. Genetic investigation result in 46 XyYcariotype, negative screening for Adrenoleucodystrophia, Peroxissomal and Wolmann Disease. Urine organic acid analysis revealed presence of glycerol. Sequencialy, labor tests showed CPK levels od 5687/10733 (normal range 38-174). Then, diagnosis of GKD was made.

Horm Res 2012;78(suppl 1) 253 
Conclusions: GKD is an Xp21 contiguous gene syndrome involving the glycerol kinase locus together with the adrenal hypoplasia congenita or Duchenne muscular dystrophy. Despite a rare disease, it maybe consi

\section{EP0-018 Adrenals and HPA Axis}

\section{Tumors of testicular adrenal rests in patients with congenital adrenal hyperplasia}

\section{Laura Ohana M. C. Carvalho; Micheline A. Rayol Souza;} Marília Martins Guimarães; Izabel Calland R. Beserra

Federal University of Rio de Janeiro, Pediatric Endocrinology, Rio de Janeiro, Brazil

Background: The growth of testicular adrenal rests tumors (TART), resulting from hyper stimulation of ACTH and angiotensin II, can be observed in individuals with congenital adrenal hyperplasia $(\mathrm{CAH})$

Objective and hypotheses: To evaluate the presence of TART in male patients with $\mathrm{CAH}$.

Methods: It was done sectional study and review of 23 years medical records of male patients with CAH. One evaluated: age at onset of puberty, Tanner pubertal stage and palpable TART presence on physical examination. Ultrasonography (USG) performed for visualization of TART and testicular lithiasis. Central precocious puberty (CPP) defined by the presence of testicular volume $>4 \mathrm{ml}$ before 9 years of age and adult height when bone age $\geq 16$ years.

Results: Twelve patients mean age of 15.36 years $(5.92-22.75)$ were evaluated, 6 salt-wasting (SW) and 6 simple virilizing (SV).

Eight $(66.7 \%)$ had completed pubertal development (Tanner G5), three $(25.0 \%)$ were G2, and one was G4. Nine $(75.0 \%)$ evolved with PPC after starting treatment and attained average adult height of $157.2 \pm 7.4 \mathrm{~cm}$. In 6 patients $(5 \mathrm{SW})$ TART was seen in USG, from those four $(66.7 \%)$ had been previously palpated on physical examination.

All were Tanner stage G5. In half of patients with TART (2SW and 1VS) was also visualized testicular lithiasis. The mean testicular volume in patients with TART was $17.3 \pm 5.9 \mathrm{~mL}$ and that one without was $17.3 \pm 3.5 \mathrm{~mL}$.

Conclusions: From 12 patients most progressed to CPP after initiation of treatment of $\mathrm{CAH}$ and reached adult height $2 \mathrm{SD}$ below the mean. TART was identified in half of patients, almost all salt-wasting. Lithiasis associated to TART was common.

These results suggest relationship between poor control and TART. The volume of the testis did not help to differentiate patients with those without TART.

\section{EP0-019 Adrenals and HPA Axis \\ Adrenal insufficiency after glucocorticoid treatment withdrawal \\ Jacobo Perez Sanchez; Raquel Corripio Collado; \\ Miriam Gonzalez Moreno; Josefa Rivera Lujan \\ Hospital de Sabadell. Corporació Sanitària Parc Taulí. Institut \\ Universitari Parc Taulí - UAB., Pediatrics, Sabadell, Spain}

Background and objective: Chronic high doses of glucocorticoids (GC) can lead to cortisol deficiency resulting from hypothalamic-pituitary-adrenal suppression despite a carefully tapering. The aim was to evaluate the adrenal suppression and time of recovery in children who received GC therapy.

Methods: We analyzed those children who received GC treatment longer than three weeks from 2008 to 2011 . We evaluated the dosage, type of GC and duration of administration. GC therapy was tapered according each indication recommendations. A short low-dose ACTH test with $1 \mu \mathrm{g}$ was performed at the end of the treatment tapering. Cortisol was measured baseline as well as 30 and 60 minutes after stimulation, and a response $>18 \mathrm{mg} / \mathrm{dl}$ was considered normal. Patients who failed the test were continued on corticoids and the test was repeated periodically every 6 months until recovery.

Results: We evaluated 10 children ( 6 male), aged between 9 months and 11 years, six of them with Cushingoid appearance. The reason for GC therapy included dermatologic problems in three of them, neurologic in three, respiratory in two, and the others cardiac or immunological problems. The median duration of glucocorticoid treatment was 3.4 months (range 21 days - 9 months) and the mean prednisone equivalent dose was $1.22(0.17-12.15)$ $\mathrm{mg} / \mathrm{kg}$.

None of them presented signs or symptoms of adrenal insufficiency. The ACTH test showed insufficient response in all of them. The median recovery of the adrenal function was at 6.9 months (range 5.5 - 14 months).
Conclusions: At usual dosage, we found adrenal suppression in all the children despite careful tapering. It's recommended to be aware of the risk of potential adrenal insufficiency, at least during the first six months after GC therapy.

\section{EPO-020 Autoimmune Endocrine Disease}

\section{Growth and puberty in celiac disease}

Mimouna Bessahraoui; Karim Bouziane-Nedjadi; Sakina Niar;

Malika Naceur; Ghazalia Boudraa; Mahmoud Touhami

Service de Pediatrie "C" CHU Oran, Medicine, Oran, Algeria

Aim: The aim of the present study was to evaluate growth, celiac disease (CD) adult height and age at menarche.

Subjects and methods:

1. Growth was assessed by:

- Longitudinal study, compared with Sempe, Pedron subjects.

- Semi longitudinal case-control study. Controls ( eliac brothers and sisters).

2. Age at menarche was assessed by case-control study. Controls (eliac sisters).

Results:

1. Growth was significantly more delayed in CD patients (103 girls, 92 boys) than in Sempe, Pedron subjects. However, evident catch-up growth was noted between the 18 and 21 years age (103 CD girls and $92 \mathrm{CD}$ boys $\mathrm{CD}$ ).

2. The growth speed was less important during puberty compared to Sempe, Pedron subjects. After 18 age, our CD patients was contuned their growth in time where Sempe, Pedron subjects finished their growth.

3. When $\mathrm{CD}$ was associated with Diabete type 1 , growth was significantly more delayed than in the $\mathrm{CD}$ isolated or diabetes patients isolated.

4. Semi-longitudinal study:

The mean adult height was: $158,42 \pm 6,3 \mathrm{~cm}(269$ women CD) vs $162,17 \pm$ $6,3 \mathrm{~cm}(193$ controls : $<<0,0001)$.

The mean adult height was $170,28 \pm 7,5 \mathrm{~cm}(194 \mathrm{men} \mathrm{CD})$ and $172,53 \pm 6,8$ cm (200 controls: $\mathrm{p}<0.09)$.

5. The mean age of menarche in $174 \mathrm{CD}$ was: $4,56 \pm 1,63$ years vs $13,74 \pm$ 1,36 years in 174 controls $(\mathrm{p}<0,0001)$.

Conclusion: Growth, puberty and adult Height are delayed especially in the $\mathrm{CD}$ girls. Additional factors influencing growth, is the auto immuns diseases associated.

\section{EP0-021 Autoimmune Endocrine Disease \\ Peculiarities of combined autoimmune endocrinopathies in children \\ Olga Zagrebaeva; Anzhalika Solntsava \\ Belarusian State Medical University, Paediatric, Minsk, Belarus}

Background: Autoimmune thyroiditis (AT), which is one of the most common cause of thyroid dysfunction, often occur with diabetes mellitus type 1 (DM1) in children.

Objective and hypotheses: Evaluate anamnesis, clinical and laboratory parameters in children with combined autoimmune endocrinopathies.

Methods: We analyzed retrospectively 232 children with AT and AT with DM1 in the endocrinological department of University hospital (Minsk) over 2002-2011 years. Group $1(\mathrm{G} 1)$ - patients with AT $(\mathrm{n}=211)$, boys(B): $\operatorname{girls}(\mathrm{G})$ $=42: 169$, mean $\pm \mathrm{SD}$ age: $11,5 \pm 2,2 \mathrm{yrs}$; group $2(\mathrm{G} 2)-$ with AT and DM1 $(\mathrm{n}=21), \mathrm{B}: \mathrm{G}=6: 15$, mean $\pm \mathrm{SD}$ age: $11,1 \pm 3,05$ yrs. Ultrasound of thyroid gland, levels of TSH, free thyroxine, Anti-TPO were analyzed. The results were processed using the Statistica 6.1.

Results: $87(41,2 \%)$ patients in G1 had positive family history on the endocrinopathies (thyroid diseases - $61(28,9 \%)$, DM2 - 22 (10,4\%), DM1 - 2 $(0,95 \%)$, other endocrinopathies $-2(0,95 \%)$ cases); in G2 - 8 (38\%) (thyroid diseases - $4(19 \%)$, DM2 - $4(19 \%)$.

The average volume of the thyroid was $138,4 \pm 40,4 \%$ G1 B, G2 B $145,3 \pm$ $39(\mathrm{p}=0,8) ; \mathrm{G} 1 \mathrm{G} 153,4 \pm 64,8 \%, \mathrm{G} 2 \mathrm{G} 171 \pm 49,3 \%(\mathrm{p}=0,2)$ from the age norms. TSH level in G1 B $12 \pm 4,2(0,23-3,4 \mu \mathrm{IU} / \mathrm{L}), \mathrm{G} 2 \mathrm{~B} 5,1 \pm 1,8 \quad(\mathrm{p}=0,9)$; in G1 G $11,5 \pm 1,9, \mathrm{G} 2 \mathrm{G} \mathrm{4,8} \pm 0,9(\mathrm{p}=0,9)$. Free thyroxine level in $\mathrm{G} 1 \mathrm{~B} 17,2$ $\pm 5(10-23,2 \mathrm{pmol} / \mathrm{L}), \mathrm{G} 2 \mathrm{~B} 16,8 \pm 2,5(\mathrm{p}=0,6)$; in G1 G $14,7 \pm 4,2, \mathrm{G} 2 \mathrm{G} 16,4$ $\pm 2,6(\mathrm{p}=0,8)$. Anti-TPO level in G1 B $507 \pm 84,8(<30 \mathrm{U} / \mathrm{ml})$, G2 B 593,3 \pm $273,5(\mathrm{p}=0,01)$; in G1 G 575,5 $\pm 55, \mathrm{G} 2 \mathrm{G} 436,3 \pm 97(\mathrm{p}=0,4)$.

In G1 $97(45,9 \%)$ patients had subclinical hypothyroidism, $74(35,1 \%)$ - euthyroid, $40(19 \%)$ - primary hypothyroidism. In G2 11 (52,4\%) - subclinical hypothyroidism, $10(47,6 \%)$ - euthyroid. 
Conclusions: AT was manifested with the development of subclinical hypothyroidism and goiter in all cases. The most pronounced decrease of thyroid function was in boys and girls in group 1 (G1-64,9\%, G2-52,4\%). The maximum thyroid volume was in group with AT and DM1 in boys and girls.

\section{EPO-022 Autoimmune Endocrine Disease \\ TYPE II polyglandular autoimmune syndrome (PASII)}

Miquel Gussinye; Maria Angeles Albisu; Diego Yeste; Maria Clemente; Ariadna Campos Martorell; Antonio Caarrascosa

Vall Hebron, Paediatric Endocrinology, Barcelona, Spain

Background: PASII is rare in children. It is a polygenic disease with autosomal dominant inheritance. The organ most frequently affected is thyroid gland( $75 \%)$, followed by pancreas $(60 \%)$ and adrenal gland( $40 \%)$. The first condition to appear is Type I Diabetes Mellitus (DMI). The relevance of the case lies in the age of onset, the short interval of time between Graves Disease(GD) and Addison Disease(AD) (4 months) and the order of occurrence of autoimmune diseases (AID).

Case report: A 6 year old boy is referred for weight loss, bilateral exophthalmos, goiter and tachycardia. The blood analysis reveals TSH $<0.004 \mathrm{mU} / \mathrm{L}$, FT4 $3.93 \mathrm{ng} / \mathrm{dL}$, TSI $20.6 \mathrm{IU} / \mathrm{L}$ Ac.anti-TPO1495IU/mL. The thyroid ultrasound is compatible with GD. He presents an allergic reaction to the antithyroid drugs (methimazole and propylthiouracil) and radioablation is performed. One month after the ablation, treatment with levothyroxine is started. $\mathrm{He}$ is admitted for asthenia and vomiting. Severe hyponatremia $(123 \mathrm{mmol} / \mathrm{L})$ and sodium loss in urine is detected as well as TSH $65.9 \mathrm{mU} / \mathrm{L}$, FT4 $8.16 \mathrm{pmol}$ / L. A differential diagnosis between SIADH and salt wasting syndrome was made as a result of hypothyroidism. After increasing the dose of levothyroxine and administering sodium, a level of $132 \mathrm{mmol} / \mathrm{L}$ of natremia is reached. A week later, the clinical symtoms reappear, but this time, thyroid hormone levels are normal and with serum sodium of $130 \mathrm{mmol} / \mathrm{L}$. The physical examination revealed generalised hyperpigmentation. $\mathrm{AD}$ is suspected and confirmed by finding levels of cortisol $<0.2 \mathrm{mcg} / \mathrm{dL}$ and ACTH $>1250 \mathrm{pg} / \mathrm{mL}$. Treatment is initiated with fludrocortisone and hydrocortisone. PAS II is suspected. After a study of antibodies is made, specific $\mathrm{Ab}$ anti adrenal gland and anti-pancreas result positive. To rule out DMI, interstitial glucose is monitored, showing normal values.

Conclusions: PASII has to be suspect if there are two AID. The study of autoantibodies is necessary to provide future involvement of other organs.

\section{EPO-023 Autoimmune Endocrine Disease \\ Bartonella henselae infection associated with autoimmune thyroiditis: a case report \\ Rosa Maria Chiuri; Ferrina Matronola; Concetta Di Giulio; \\ Francesco Chiarelli; Annalisa Blasetti \\ University of Chieti, Department of Pediatrics, Chieti, Italy}

Background: Bartonella henselae was discovered a quarter of a century ago as the causative agent of cat-scratch disease. Most recently Bartonella has been found to be responsible for a broad range of clinical syndromes (prolonged fever, hepatosplenic disease, encephalopathies, ocular disease) and associated with autoimmune conditions, particularly, haemolytic anemia, thrombocytopenic purpura, systemic juvenile rheumatoid arthritis, systemic lupus erythematous, glomerulonephritis, Guillain-Barrè syndrome.

Objective and hypotheses: This is the first report of autoimmune thyroiditis related to Bertonella henselae infection.

Population and/or methods: We describe an 11-year-old boy who presented with goiter and weight loss. Laboratory tests showed an increase in thyroid hormones with TSH suppression and significant increase in Anti-thyroglobulin, Anti-thyroperoxidase and Anti-TSH receptor antibodies titers. At the time of admission a $2 \times 1 \mathrm{~cm}$ mildly tender right supraclavicular lymph node was noted in association with an erythematous papule at the same side of the neck. Serological data showed a high titer of Bartonella henselae IgG (1:100).

Results: We made a diagnosis of autoimmune hyperthyroidism in association with cat-scratch disease. Therefore therapy with tapazole $(10 \mathrm{mg} /$ day $)$ and clarithromycin $(15 \mathrm{mg} / \mathrm{kg} /$ day $)$ was started. We have documented a gradual normalization of thyroid hormones and regression of lymphadenopathy and erythematous lesion of the neck in two weeks.

Conclusions: Different types of infections are implicated in the pathogenesis of autoimmune thyroid diseases through molecular mimicry or other mecha- nisms, despite their role is disputed. We report the first case of autoimmune thyroiditis in a child infected with Bartonella henselae. The immune response to this bacillus infection may be associated with the development of autoimmune disorders. We speculate that autoimmune thyroiditis should be added to the spectrum of clinical syndromes that can be triggered by Bartonella henselae.

\section{EPO-024 Autoimmune Endocrine Disease}

\section{Prevalence and follow-up of thyroid disfunction at type 1 diabetes mellitus (T1DM) onset in pediatric population}

Claudia Balsamo; Alessandra Cassio; Stefano Zucchini;

Mirella Scipione; Giulio Maltoni; Alessandra Rollo; Annalisa Martini;

Diego Rinaldini; Angela Rizzello

S.Orsola Malpighi Hospital - University of Bologna, Pediatric Clinic -

Endocrine Unit, Bologna, Italy

Background: The nonthyroidal illness syndrome (NTIS) is a condition characterized by abnormal thyrotropin and thyroid homone levels and it's frequently reported during severe illness. T1DM onset is an acute stressful event and could affect thyroid function.

Objective and hypotheses: To study thyroid function at T1DM onset and after 1-90 days.

Methods: We examined 149 patients $(72 \mathrm{M}$ and $77 \mathrm{~F}$, mean age $7.7 \pm 4.1 \mathrm{yrs}$ ) diagnosed for T1DM from 2000 to 2011. 20 pts were anti-thyroid autoantibodies positive at T1DM diagnosis. The 129 pts without autoimmunity were subdivided in the following group: 63 had abnormalities in thyroid hormone levels ( 8 pts had hypertireotropinemia, 30 pts had low fT3 levels, 23 pts had both low fT3 and fT4 and 2 pts had suppressed TSH too) and the remaining 66 were normal

Results: Multiple regression analysis identified BE $(R 2=0.38 ; \mathrm{p}<0.0001)$ and fructosamine $(\mathrm{R} 2$ change $=0.12 ; \mathrm{p}<0.0001)$ as major influencing variables on fT3 levels. Table 1 shows parameter at diabetes onset.

\begin{tabular}{lccc} 
& $\begin{array}{c}\text { Normal thyroid function } \\
(\mathbf{7 7} \text { pts) }\end{array}$ & $\begin{array}{c}\text { Pathological thyroid } \\
\text { function }(72 \text { pts })\end{array}$ & $\mathbf{p ~}$ \\
\hline Weight SDS & $-0.06 \pm 0.9$ & $-0.52 \pm 0.9$ & $<0.006$ \\
\hline BMI SDS & $-0.28 \pm 1$ & $-1.02 \pm 1.1$ & $<0.0001$ \\
\hline HbA1c (\%) & $11.14 \pm 2.3$ & $12.5 \pm 2.1$ & $<0.0001$ \\
\hline Fructosamine (ìmol/l) & $559.27 \pm 135.2$ & $680.73 \pm 146.8$ & $<0.0001$ \\
\hline pH & $7.32 \pm 0.1$ & $7.22 \pm 1.3$ & $<0.0001$ \\
\hline Glycemia (mg/dl) & $396.35 \pm 182.9$ & $500 \pm 204.5$ & $<0.001$ \\
\hline BE & $-3.95 \pm 8.4$ & $-12 \pm 10$ & $<0.0001$ \\
\hline Aldosteron (ng/dl) & $271.55 \pm 238$ & $760.3 \pm 751.4$ & $<0.0001$ \\
\hline Cortisol (ig/dl) & $155.76 \pm 84.6$ & $240.3 \pm 133.6$ & $<0.0001$ \\
\hline ACTH $(\mathrm{pg} / \mathrm{ml})$ & $21.04 \pm 15.5$ & $21.8 \pm 45.9$ & $<0.01$ \\
\hline C-peptide (ng/ml) & $0.73 \pm 0.5$ & $0.46 \pm 0.3$ & $<0.0001$
\end{tabular}

In all subjects thyroid levels became normal after 1-90 days.

Conclusions: Thyroid disfunction, in particulary NTIS, is frequent at T1DM diagnosis regardless of autoantibodies. Thyroid function is strictly related to seriousness of metabolic pattern. A delayed diagnosis could compromise not only metabolic balance but also thyroid function.

\section{EPO-025 Autoimmune Endocrine Disease}

\section{A case of incomplete precocious puberty due to a severe and long-lasting primary hypothyroidism}

Rossella Gaudino; Grazia Morandi; Francesco Doro; Giulia Rodella; Evelina Maines; Claudia Piona; Laura Tenero; De Luca Giuseppina; Antoniazzi Franco

University of Verona, Department of Life and Reproduction, Verona, Italy

Background: Van Wyk-Grumbach syndrome (VWGS) is a rare condition, characterized by juvenile acquired hypothyroidism, delayed bone age, and isosexual precocious puberty. It usually appears in girls, who have also cystic ovarian enlargement but also boy can be interested. 
Case report: We describe a boy, 5 years old, coming to our clinic for a marked growth reduction since the age of 3 . He presented dry skin and brittle hair, with a history of constipation, poor reactivity and fatigue in the fine movements. At the visit he had a height $<3$ SDS with a weight at $-1 /$ M SDS and with both testis increased in volume for age $(4 \mathrm{ml}$ the right and $5 \mathrm{ml}$ the left one). The blood sample revealed a clear hypothyroidim: fT3: $0.9 \mathrm{pmol} / \mathrm{L}$ (VN: 3.9-6.8), fT4: 1.19 pmol/L (VN 8-20), TSH: $1493 \mathrm{mU} / \mathrm{L}$ (VN 0.3-5), Ab anti-TPO: $2659 \mathrm{kU} / \mathrm{L}$ (VN 0-16), Ab anti-TG: $404 \mathrm{kU} / \mathrm{L}$ (VN 0-100). A thyroid ultrasound was performed and confirmed a case of Hashimoto's thyroiditis. The bone age was of 2 years according to Greulich e Pyle Atlas. To investigate the macroorchidia we performed a $\mathrm{GnRH}$ analogue test, which resulted negative for a central precocious puberty. A therapy with L-tyroxine was immediately started with a relevant improvement of the clinical condition of the child and the resolution of the testis enlargement in the first six months. Conclusion: Many theories have been proposed to explain the relationship between a primary hypothyroidism and a peripheric precociuos puberty, according to the most recognized one TSH in high concentrations may stimulate FSH receptors in the gonads. It's thus important not to forget thyroid assessment in case of signs of precocious puberty.

\section{EPO-026 Bone, Growth Plate and Mineral Metabolism}

\section{Infantile malignant osteopetrosis: a rare cause} of neonatal hypocalcemia

Ozlem Engiz'; Semra Kara²; Denizhan Bagrü; Georgia Lahr Inci Arikan ${ }^{3}$; Yíldíz Bilge Dallar ${ }^{3}$

${ }^{1}$ Ankara Training and Research Hospital, Pediatric Endocrinology,

Ankara, Turkey; ${ }^{2}$ Ankara Training and Research Hospital, Neonatology,

Ankara, Turkey; ${ }^{3}$ Ankara Training and Research Hospital, Pediatrics,

Ankara, Turkey; ${ }^{4}$ University of UIm, Pediatrics and Adolescent

Medicine, Ulm, Germany

Background: Infantile malignant osteopetrosis (IMO) (OMIM 259700) is a rare inherited bone disease characterised by reduced or dysregulated activity of osteoclasts, resulting in generalised osteosclerosis. The disease usually presents within the first few months of life with anemia, hepatosplenomegaly, frontal bossing, nystagmus, blindness, deafness, and bone fractures. Children with IMO are at risk of developing hypocalcaemia, with attendant tetanic seizures.

Results: We report the case of a baby boy who presented with hypocalcemia on day 1. Oral calcium lactate and calcitriol treatments were started. Skeletal radiographs demonstrated uniformly dense, homogenous and sclerotic bones and a dense base of the skull with typical "space alien" face confirming the diagnosis of IMO. Pancytopenia developed at 2 months of age. Visual evoked potential showed severe bilateral optic nerve damage. Genetic mutation study revealed a mutation in exon 11 of the TCIRG1 gene.

Conclusions: Neonatal hypocalcemia can occur as result of IMO which is easily missed out by clinicians. This causes delay in establishing the diagnosis and starting necessary treatment. Therefore, osteopetrosis should be kept in mind as a rare cause of neonatal hypocalcemia.

\section{EPO-027 Bone, Growth Plate and Mineral Metabolism Congenital hypomagnesaemia a case report Jridi Ines; Barakizou Hager; Barrak Sarra; Gannouni Souha; Bayoudh Fethi \\ Military Hospital, Paediatrics, Tunis, Tunisia}

Background: Congenital hypomagnesaemia is an autosomal recessive disease, although it might be in some cases a sex-linked recessive disease, more prevalent among men than women. It manifests itself, the first weeks of life, by severe and frequent seizures and tetany.

Objective: It's due to the intestinal malabsorption of magnesium resulting from a lack of jejunal transfert.

Methods: Here we report the case of a boy born to second-degree consanguineous parents, by cesarean section after a full term pregnancy and with a good adaptation to extra -uterine life. He presented at the age of 2 months generalized clonic seizures accompanied with hypocalcaemia refractory to calcitherapy. The rest of the record reveals a deep hypomagnesaemia with the rate of $0.44 \mathrm{mmol} / 1$ and without hypermagnesuria.

Results: The evolution was favorable under supplementation with magnesium and calcium but marked by the recurrence of the same symptomatology in case of gastroenteritis or interruption of the treatment. The confirmatory diagnosis of congenital hypomagnesaemia is through an oral or parenteral magnesium load test. The treatment remains a lifetime magnesium supplementation. With hindsight of 8 years 4 months, the child has a correct psychomotor development, proper schooling and a good height and weight growth in a well conducted treatment.

Conclusion: Congenital hypomagnesaemia is rare and must be evocated in case of refractory seizures with hypocalcemia

EP0-028 Bone, Growth Plate and Mineral Metabolism

\section{A heterozygous deletion of at least $128 \mathrm{~kb}$ affecting RUNX2 causes cleidocranial dysplasia in an 11-year old girl and her father G. Gemulla'; C.E. Ott²; N. Di Donato3; A. Hübner}

'Technical University Dresden, University Children's Hospital,

Dresden, Germany; ${ }^{2}$ Charité-Universitätsmedizin Berlin, Institute for

Medical Genetics and Human Genetics, Berlin, Germany; ${ }^{3}$ Technical

University Dresden, Institute of Clinical Genetics, Dresden, Germany

Background: In patients with Cleidocranial Dysplasia (CCD) point mutations of $R U N X 2$ are found in $2 / 3$ of all cases. The frequency of deletions is estimated to be around $10 \%$.

Objective and hypotheses: The patient was referred to the endocrine department because of short stature. Physical examination showed missing acromial ends of both claviculae, gothic palate, micrognathia and abnormal dentition. Until now no permanent teeth did break through. Closure of the anterior fontanel was retarded. Numerous upper airway infections occurred in infancy. An adenotomy was performed. Mental development was normal. Family history revealed similar symptoms in an older sister and the father suggesting autosomal dominant inheritance. Growth hormone deficiency, hypothyroidism, hypercortisolism, hyperprolactinemia, disorders of calcium/phosphate metabolism, liver and kidney dysfunction were excluded. Karyotype was 46,XX. Methods: As clinical assessment was indicative for Cleidocranial Dysplasia, we performed analysis of $R U N X 2$.

Results: Sanger sequencing of all coding exons did not show any point mutations in RUNX2 but quantitatitive PCR detected a heterozygous deletion of at least $128 \mathrm{~kb}$ spanning exons 2 to 8 in the girl and her father. The aberration size was not investigated further as no contiguous gene syndrome was suspected. So far, examination of the older sister has not been possible.

Conclusions: Deletion/duplication analysis should be performed if clinical features suggest CCD and molecular genetic analysis of $R U N X 2$ yields negative results. We report a deletion of at least $128 \mathrm{~kb}$ affecting $R U N X 2$ which leads to Cleidocranial Dysplasia in an 11-year old girl and her father.

\section{EPO-029 Bone, Growth Plate and Mineral Metabolism}

\section{Effects of levothyroxine replacement therapy of congenital hypothyroid children on their bone mineral density}

Shadab Salehpour'; Saeed Tavakkoli'; Somayeh Setavand ${ }^{3}$

${ }^{1}$ Research Institute for Endocrine Sciences, Endocrinology, Tehran, Islamic Republic of Iran; ${ }^{2}$ Pediatric Orthopedics, Milad General Hospital, Tehran, Islamic Republic of Iran; ${ }^{3 P S R C}$, Pediatrics, Tehran, Islamic Republic of Iran

Objective: To determine the effects of long-term with levo-thyroxine replacement therapy on bone mineral density in children with congenital hypothyroidism

Method: 34 children (22 girls and 12 boys) at 5 years old who had been diagnosed as $\mathrm{CH}$ based on the national newborn screening program and had been taking levothyroxine since their first month of life were selected base on the random allocation. The bone mineral density, bone area, bone mineral content and bone mineral apparent density of the femoral neck, lumbar vertebra and whole body were measured by dual energy X-ray absorptiometry and calculated, and compared with those of the age and sex-matched children in the control group.

Results: After correcting the confabulating factors like calcium and vitamin $\mathrm{D}$ intake, there was no significant difference between $\mathrm{CH}$ children and the control group in BMD and BMAD.

Conclusion: Replacement therapy with levothyroxine in congenital hypothyroid children has no influence on their bone mineral density. 


\section{EPO-030 Bone, Growth Plate and Mineral Metabolism}

Familial case of pseudohypoparathyroidism

Natalija Smetanina; Rasa Verkauskiene; Edita Jasinskiene;

Rimante Dobrovolskiene; Indre Petraitiene; Rima Berontiene;

Giedre Mockeviciene

Lithuanian University of Health Sciences, Medical Academy,

Department of Pediatric Endocrinology, Kaunas, Lithuania

Background: Pseudohypoparathyroidism is a heterogeneous group of disorders caused by molecular defects in the GNAS1 gene encoding the alpha subunit of the stimulatory G protein (Gsa).

Objective and hypotheses: The aim of this study is to present a clinical case of familial pseudohypoparathyroidism in 3 individuals of the same family. Methods: Description of the clinical case of familial pseudohypoparathyroidism.

Results: We present a family with 3 children. The oldest boy of $14 y 5 \mathrm{~m}$ age complained of the left eye twitching for the last 4 months, two other sibs (13y $6 \mathrm{~m}$ boy and $11 \mathrm{y} 11 \mathrm{~m}$ girl) had any complaints at the moment of the diagnosis All of them had similar phenotype: plump, round face, short neck, dysplastic teeth enamel, short stature, short hands and feet, normal puberty and positive Chvostek sign. High levels of PTH concentration $(35,2 \mathrm{pmol} / 1,58,5 \mathrm{pmol} / 1$ and $87,2 \mathrm{pmol} / \mathrm{l})$ and low levels of ionized calcium $(0,56 \mathrm{mmol} / 1,0,54 \mathrm{mmol} / \mathrm{l}$ and $0,64 \mathrm{mmol} / \mathrm{l}$ ) were found in the older and younger boys and the girl, respectively. Elevated TSH and low FT4 were found in all 3 children. Calcifications in the basal ganglia were found in the brain CT scan images also in all sibs. All children had learning difficulties at regular school.

The mother's phenotype was similar to children. However, her TSH, FT4 and PTH hormones levels were normal. Father had normal phenotype; his hormone levels were not evaluated.

Conclusions: We described the familial case of pseudohypoparathyroidism in all 3 children in one family. If pseudohypoparathyroidism is found in one child all other children should be also screened for this disorder.

\section{EP0-031 Bone, Growth Plate and Mineral Metabolism \\ A novel missense mutation in GNAS in exon 10 (p.A243V) associated with pseudohypoparathyroidism type Ic Susanne Thiele $^{1}$; Dagmar Struve ${ }^{2}$; Olaf Hiort ${ }^{2}$}

1University of Luebeck, Pediatrics and Adolescent Medicine, Luebeck, Germany; '2University of Luebeck, Pediatrics, Luebeck, Germany

Background and objective: Pseudohypoparathyroidism (PHP) comprises a heterogeneous group of disorders defined by PTH resistance. PHP type Ia (PHPIa) is caused by GNAS mutations leading to deficiency of the álphasubunit of stimulatory G proteins (Gs-álpha) that mediates signal transduction of G protein-coupled receptors via cAMP synthesis. PHP type Ic (PHPIc) and PHPIa share clinical features including Albright hereditary Osteodystrophy; however, in contrast to PHPIa, in-vitro activity of solubilized Gs-álpha protein measured in erythrocyte membranes is not diminished in PHPIc. Some inactivating GNAS mutations have been described in PHPIc, which were all located in the extreme carboxy-terminus of Gs-álpha encoded by exon 13, selectively affecting receptor coupling of the protein. Patient: A 13 year old boy presented with elevated PTH of $174 \mathrm{pg} / \mathrm{ml}$ (15-55), obesity and brachymetacarpia.

Methods and results: Gs-álpha protein activity was $101 \%$, compared to normal (85-115). GNAS sequencing revealed a sporadic missense mutation in exon 10 (c.728C $>$ T, p.A243V). The mutation was introduced into a pcDNA3.1. vector encoding Gs-álpha-wild type and expressed in a Gs-álphaknock out cell line (Gnas E2-/E2-). To investigate receptor-mediated cAMP accumulation, we stimulated the endogenous expressed â2-adrenergic receptor and demonstrated diminished cAMP synthesis by a RIA, compared to the wild type.

Conclusion: In this study, we describe a novel GNAS mutation leading to PHPIc, which is for the first time not located in the extreme carboxyl-terminal part of the protein.

\section{EPO-032 Bone, Growth Plate and Mineral Metabolism \\ Calcium intake during adolescence, is food enough? \\ Shokery Awadalla \\ Hospital san Jose, Pediatric Endocrinology, Bogota, Colombia}

Background: Calcium intake during the growing years of adolescence is one of the main factors determining future bone health.

Objective and hypotheses: the aim of the study was to evaluate The sufficiency of calcium intake from food and the need of calcium supplementation at this age.

Methods: 240 healthy children, who consulted for routine medical evaluation, 130 girls and 110 boys, were included. Food questionnaires to the parents were recorded during one week and the mean of calcium intake from diary and non-dairy products was calculated .Percentage of intake and recommended needs were compared.

Results: Age was $12 \pm 1.2$ years in girls and $14 \pm 0.9$ years in boys and body mass index was between 25 and 75 percentile in both. Pubertal development was Tanner 2-3. No relevant fracture history was observed. Milk, cheese and Yogurt, were the main calcium sources (60\% in girls and $75 \%$ in boys, $\mathrm{p}<$ $0.05)$. Green vegetables, nuts and fish accounted for $40 \%$ in girls and $25 \%$ in boys. 32 girls $(25 \%)$ and 44 boys ( 40 ) had calcium intake near $1300 \mathrm{mg}$ / daily, the rest 98 girls ( 75 ) and 66 boys ( $60 \%$ ) had mean calcium intake $550 \mathrm{mg} / \mathrm{d} \pm 200$ in girls and $750 \mathrm{mg} / \mathrm{d} \pm 250$ in boys $(\mathrm{p}<0.05)$, almost half the recommended daily needs at this age . Carbonated drinks were consumed by $72 \%$ of the group and $30 \%$ drank $1-2$ units daily. Dairy products were the main calcium source in the studied group, especially in boys, less in girls for fear of overweight.

Conclusions: Only $25 \%$ of girls and $40 \%$ of boys have adequate calcium intake, Questionnaire about calcium intake should be a routine in patient visit and supplementation if necessary thus insure healthy bone formation

\section{EPO-033 Bone, Growth Plate and Mineral Metabolism \\ Vitamin D receptor gene - a necessity for diagnostic in calcium and bone metabolism disorders? \\ Luminita Beldean'; Wolfgang Hoeppner ${ }^{2}$ \\ 1"Lucian Blaga" Univeristy from Sibiu, Faculty of Medicine, Sibiu, \\ Romania; ${ }^{2}$ Bioglobe Ltd, Genetics, Hamburg, Germany}

Background: In 1998 Hughes identified two different mutations of VDR in two patients with vitamin D- dependent rickets type II. Giguere and Roussea, (2000), stated that twin studies had shown how genetic factors account for up to $80 \%$ of the variance in bone mineral density. Fang, 2005, studied VDR gene in 937 cases with clinical fractures recorded in a follow up of 7 years. In a number of 16 subjects who had 2 VDR mutations, fracture risk increased to $48 \%$, independent of age, height, weight, bone mineral density.

Objective: To search familial and genetic background of cases with persistent clinical signs/symptoms of hypocalcemia. Hypotheses: looking carefully at family hystory data we can find out if there is evidence of calcium metabolism disorder inheretance.

Methods: Cases were selected from the patients referred to the endocrinology department (2010-2011) by family doctors because of persistent symptoms/ signs of hypocalcemia. Patients aged: from 16 to 50 years. Laboratory tests and imagistic data were collected. Selected cases have no evidence of liver or kidney pathology, no hypoparathyroidism, no diet or sun-exposer deficit. Follow-up treatment with vitamin D.

Results: Group 1: 50 patients - familial evidence of hypocalcemia, clinical signs/symptoms as: numbness, tingling, muscle cramps, fasciculations, irritability, Chwostek sign. In 3 cases patients had both fractures in childhood and osteoporosis before menopausal age. Family background induces the idea of genetic defect. Group 2 (part of group 1): seven subjects accepted to have vitamin D receptor gene test (DNA). There is evidence of defects in codon 1 and intron 8 . Treatment with vitamin D (up to 10.000 units) contributed to the patients' symptoms relief.

Conclusion: Case studies and bibliografical data demonstrate that family history and genetic tests can be good support for further diagnostic/research in calcium and bone metabolism disorders. 


\section{EPO-034 Bone, Growth Plate and Mineral Metabolism}

Idiopathic hypoparathyroidism - an "iceberg" of ca sensing receptor gene defects?

Luminita Beldean; Corina Roman-Filip

"Lucian Blaga" Univeristy from Sibiu, Faculty of Medicine, Sibiu,

Romania

Background: Finegold et al. 1994 presented evidence for linkage of a form of autosomal dominant hypoparathyroidism to a region of 3q13 (Ca sensing receptor gene). In 1996 Baron identified 2 families wirh autosomal dominant hypoparathyroidism with heterozigous mutation the CaSR gene. Tan, 2003, reported a three-generation family with autosomal dominant hypocalcemia (single base transition in exon 7 of CaSR). Whereas all affected individuals exhibited marked hypocalcemia, some cases with untreated hypocalcemia exhibited seizures in infancy, and others were largely asymptomatic from birth into adulthood. Kappor et al, 2008, by genome wide linkage and candidate gene sequencing of a large Indian Family with idipatic generalized epilepsy, mapping the chromosome 3Q12.3-q21, identified a mutation in the CaSR gene.

Objective and hypotheses: To underline the role of family assessment in cases diagnosed with idiopatic hypoparathyroidism. To warn for a careful evaluation of calcium metaboilsm disorders in cases which associate seizure and hypocalcemia.

Methods: Case study; selection of the patients referred to the endocrinology department because of persistent hypocalcemia and hystory of seizure. Clinical findings, personal and familial hystory, laboratory tests were collected from the patients in the study.

Results: 4 patients (1 girl, 3 boys) with hypoparathyroidism, diagnostic age: 5 -12 years old. All cases experienced seizure, 2 of them treated as epilepsy: one girl for 3 years (between 1-3) and one boy for 9 years (between 3-12), 2 boys were diagnosed at the age of 7 when they presented an afebril seizure. Two cases have evidence of inheritance: autosomal dominant ? No genetic tests due to costs.

Conclusions: Cases having calcium regulation disorder happened to be treated as epilepsy. More research is necessary in families whose members experienced seizure, including febrile seizures, because the bibliografical data had shown evidence that $\mathrm{Ca}$ sensing receptor defect is one of the ethyopatogenic factors.

\section{EPO-035 Bone, Growth Plate and Mineral Metabolism}

\section{Prevalence of vitamin D deficiency in children} and adolescents attending multidisciplinary

\section{pediatric clinics}

Ibrahim Al Alwan' ; Motasim Badri'; Fadia Al Buhairan²,

Mohamed Akram ${ }^{3}$; Faris Al Enizi ; Waleed Tamimi; ${ }^{3}$ Raed Kanan ${ }^{3}$

${ }^{1}$ King Saud bin Abdulaziz University for Health Sciences, College of Medicine, Riyadh, Saudi Arabia; ${ }^{2}$ King Abdulaziz Medical City, Pediatrics, Riyadh, Saudi Arabia; ${ }^{3}$ King Saud bin Abdulaziz University for Health Sciences, College of Applied Medical Sciences, Riyadh, Saudi Arabia

Background: Vitamin D deficiency is a major risk for cardiovascular heart disease and is widely common in the Kingdom of Saudi Arabia.

Objective: To estimate the prevalence of vitamin D deficiency among Saudi children and adolescents attending different pediatric specialty clinics.

Methods: Laboratory and clinical data were collected between January to December 2009 for children and adolescents aged 17 years and younger visiting pediatric clinics. Patients with renal or bone diseases or any relevant diseases to vitamin D were excluded. Serum levels of alkaline phosphatase (ALP), albumin, calcium, phosphorus, blood urea (BUN), creatinine 25-hydroxyvitamin D and parathyroid hormone (PTH) were determined were measured.

Results: The study included 586 children and adolescents. Of these 310 were children aged less than or equal 12 years; 147 (47\%) female and 276 adolescents (aged 13-17 years); with 185 (67\%) being female. The prevalence of vitamin D deficiency $(<37 \mathrm{mmol} / \mathrm{L})$ was $37 \%$ and $69 \%$ among children and adolescents respectively. There was a significant difference $(p<0.004)$ in the prevalence of Vitamin D deficiency between female children $(20 \%)$ compared to female adolescents $(50 \%)$, but not among males $(\mathrm{p}=0.4)$. Among the adolescents, there was a significant difference $(\mathrm{p}<0.003)$ in vitamin D deficiency between females (50\%) and males (19\%). Hyperparathyroidism was found in $75 \%$ of all children and. However, only $35 \%$ of adolescents male had hyperparathyroidism.
Conclusions: The prevalence of vitamin D deficiency in Saudi children and adolescents is high, particularly among girls.

\section{EP0-036 Bone, Growth Plate and Mineral Metabolism \\ Sanjad Sakati syndrome (hypoparathyroidism- retardation- dysmorphism syndrome)}

Abeer Atef Al Ashmawy ${ }^{1}$; Sameh Tawfik'; Mona Attia';

Makarem Ibrahim 3 ; Reem Fouad 3 ; Thorya El Mahdi'; Maha Hassan ${ }^{3}$

${ }^{1}$ Cairo University, Pediatrics, Cairo, Egypt; ${ }^{2}$ Maadi Hospital, Pediatrics,

Cairo, Egypt; ${ }^{3}$ Ministry Of Health (NHS), Pediatrics, Cairo, Egypt

Background: Sanjad Sakati Syndrome is an Autosomal Recessive disorder found exclusively in people of Arabian origin. It was first reported from the Kingdom of Saudi Arabia in 1988. In Egypt, no precise data are available.

Objective: To report an Egyptian patient with Sanjad Sakati Syndrome(SSS). Methods: First case report for an Egyptian patient with SSS. A 5.5 year-old boy presented at age of 40 days with generalized convulsions. He was born to a non consanguineous parents after an uneventful pregnancy with birth weight of $1.200 \mathrm{Kgm}$. He was the 2 nd child of the family. He has six male cousins who have mental retardation, bone deformities, convulsios and deafness. The syndrome compromises of congenital hypoparathyroidism (serum calcium $6.2 \mathrm{mg} / \mathrm{dl}(8.5-10.2)$, serum phosphorus $5.1 \mathrm{mg} / \mathrm{dl}$ (4.5-5.5), alkaline phosphatase 442U/L(100-550) and serum parathormone $6.6 \mathrm{mg} / \mathrm{dl}(8.5-10.2)$, growth retardation(height SDS at the age of two years was -3.5$)$ low IQ (80\%), recurrent otitis media, recurrent pathological fracture and dysmorphism(deepset eyes, depresed nasal bridge, high arched palate) The child had recurrent admissions to hospital with convulsions, fever, sepsis and severe bleeding. The skeletal survey was normal; while there was nephrocalcinosis in pelviabdominal ultrasonography dignosed at age of 5years. Supportive treatment in the form of 1 alfa- hydroxy, vitamin D3 $(.1 \mu \mathrm{g} /$ day) and oral calcium $(70 \mathrm{mg} /$ $\mathrm{kg}$ /day) with mild clinical and metabolic improvment. Eight months ago, he was receiving parentral calcium and Teriparatide (parathormone) asocciated with marked clinical and metabolic improvment, IQ and growth. On follow up; the patient had no convulsions with serum calcium $9.2 \mathrm{mg} / \mathrm{dl}$ and phosphate $3.9 \mathrm{mg} / \mathrm{dl}$. Height SDS at age of 5.5 years was -1.7 . DNA analysis is pending to confirm the diagnosis.

Conclusion: This is the first report of Sanjad Sakati syndrome in Egypt. Early Diagnosis will provide proper counselling of the families, proper treatment and prevent associated comorbidities.

\section{EPO-037 Bone, Growth Plate and Mineral Metabolism \\ Bone mineralization and body composition in children born preterm}

Annalisa Calcagno ${ }^{1}$; Annalisa Gallizia'; Flavia Napoli' ; Enrica Bertelli'; Irene Olivieri'; Giorgia Brigati' ; Luca Ramenghi'; Mohamad Maghnie'; Natascia Di lorgi'

IIRCCS G. Gaslini Insitute, Department Of Pediatrics, Genova, Italy; ${ }^{2}$ RCCS G. Gaslini Insitute, Department of Neonatal Intensive Care, Genova, Italy

Background: Data on bone mineral density, fat mass (FM) and insulin resistance (IR) in children born prematurely compared to those born at term are not univocal.

Objective and hypotheses: Aim of our study was to evaluate body composition (bone, fat, lean mass) and IR in preterm children and to analyse their relations with early risk factors.

Methods: We enrolled 30 children ( $8 \mathrm{~F}, 22 \mathrm{M}$; mean age 5,93 $\pm 0,66 \mathrm{yrs}$ ) born at a mean gestational week (GW) of $31,7 \pm 2,4$ in an observational cross-sectional study; mean birth weight (BW) was $1788,3 \pm 436,2 \mathrm{~g}$, 6 neonates were IUGR and none was SGA. Eleven mothers were treated with prophylactic steroids (MS). Children underwent anthropometric, glucose, insulin, HBA1c, bone age and Dual X-ray absorbiometry evaluations: measures of total body (TB) without-head and lumbar (L) BMD (g/cm2, Z-score), BMC (g) and of TB FM and fat free mass (FFM) (kg, \%) were obtained.

Results: Both genders showed normal height and BMI SDSs. All TB and $\mathrm{L}$ parameters were in the normal range compared to normative DXA data; yet, girls had lower TB BMD-Z-score compared to boys $(-0,54 \pm 0,64$ vs $0,30 \pm 0,80, \mathrm{P}<0.05)$. TB parameters were lower in IUGR subjects ( $\mathrm{P}$ 's range $0,05-0,07)$ and in those with MS (P's range $0,08-0,09$ ). There was no difference in bone parameters between breastfed or artificially fed children. Males showed more FFM and FM than females $(\mathrm{P}=0,08)$ and those with MS less 
FFM than the untreated ones. No glycometabolic abnormalities were found FFM was related to all bone parameters in both genders (r's range 0.47-0.96, all $\mathrm{P}$ ' $<<0.05$ ), while BW and GW were associated with TB BMD and BMC ( $r$ 's range $0.63-0.93, \mathrm{P}<0.05$ ) in females but not in males. BW was related to $\% \mathrm{FM}(\mathrm{r}=0.54, \mathrm{P}<0.05)$ and inversely to $\% \mathrm{FFM}(-0.46, \mathrm{P}<0.05)$ in males. Conclusions: Our preliminary data indicate that prematurity is not a risk factor for osteoporosis, cardiovascular diseases or diabetes during the first 5 years of life; early determinants as IUGR and steroid prophylaxis could however play a role.

\section{EPO-038 Bone, Growth Plate and Mineral Metabolism \\ Vitamin D and hyperbilirubinemia in neonate \\ Mehmet Mutlu'; Atilla Cayir $^{2}$; Yasemin Cayir'; Behzat Ozkan³; Yakup Aslan ${ }^{4}$ \\ ${ }^{1}$ Erzurum Bölge Training and Research Hospital, Neonatal Care Unit, Erzurum, Turkey; ${ }^{2}$ Ataturk University, Pediatric Endocrinology, Erzurum, Turkey; ${ }^{3}$ Istanbul Medeniyet University, Pediatric Endocrinology, Istanbul, Turkey; ${ }^{4}$ Karadeniz Technical University, Neonatal Care Unit, Trabzon, Turkey}

Background: Low antioxidant system may contribute to the severity of neonatal hyperbilirubinemia. This study was performed with the aim of establishing whether there is a relationship between plasma vitamin D level and the severity of hyperbilirubinemia in full-term neonates.

Objective and hypotheses: Our aim in this study was to investigate whether there is a correlation between indirect bilirubin and serum vitamin D levels in newborns with jaundice at a level necessitating phototherapy.

Methods: This prospective study was performed by comparing serum vitamin D levels in newborns with a pathological level of hyperbilirubinemia and healthy newborns with a physiological level of hyperbilirubinemia or without jaundice. Newborns with postnatal ages of 3-10 days and gestational ages of 35-40 weeks, with a bilirubin level above the limit for phototherapy were enrolled in the study (Group I). Physiologically healthy newborns with similar demographic characteristics but no jaundice were enrolled as the control group (Group II). This study was performed with 51 newborns, a 30-member study group (Group I) and 21-member control group (Group II). Ethical committee approval was obtained and informed consent forms received from babies' families.

Results: Mean serum $25(\mathrm{OH})$ vitamin D in the study group was $10.7 \pm 4.9 \mathrm{ng} /$ $\mathrm{mL}$. In the control group, mean serum $25(\mathrm{OH})$ vitamin D level was $15.7 \pm 4.9$. A statistically significant different difference was determined between case and control group serum $25 \mathrm{OH}$ vitamin $\mathrm{D}$ levels $(\mathrm{p}=0.01$ ). A significant negative correlation was established between serum vitamin $\mathrm{D}$ and serum parathyroid hormone (PTH) levels in the study group cases (r:-0.3,p:0.03).

Conclusions: Our results indicate that low level of plasma vitamin D is associated with significant hyperbilirubinemia in full-term neonates. Decreased antioxidant capacity in vitamin D deficiency or insufficiency may be compensated for by an increase in bilirubin

\section{EPO-039 Bone, Growth Plate and Mineral Metabolism \\ A case of vitamin D deficiency and heart failure Francesco Doro'; Grazia Morandi'; Elena Monti' ; Paolo Cavarzere'; Michela Fedrizzi'; Lauriola Silvana'; Egidio Barbi'; \\ Alessandra Benettoni3; Rossella Gaudino'; Franco Antoniazzi' \\ ${ }^{1}$ University of Verona, Department of Life and Reproduction, Verona, Italy; ${ }^{2 B}$ urlo Garofalo, Pediatric Clinic, Trieste, Italy; ${ }^{3 B}$ Burlo Garofalo, \\ Cardiology, Trieste, Italy}

Introduction: The association between severe Vitamin D deficiency and heart failure is described in literature, but in adults only.

Objective: This case report observes the effects of vitamin D supplementation in a subject with heart failure and severe rickets.

Case report: We report a case of a one-year old infant, admitted to the paediatric intensive care unit for cardiac insufficiency. The child is of African ethnicity, exclusively breastfed without vitamin D supplementation both for child and for mother. At the physical examination the infant appeared wakeful but reactive, normal length and weight, $\mathrm{AF} 3 \times 3 \mathrm{~cm}$, costochondral swelling (rachitic rosary) at thorax exam, varus deformity of lower limbs, ankles widening (double malleoli sign), frontal bossing and no Harrison's groove. Laboratory tests showed a severe deficiency of Vitamin D $(<10 \mathrm{nmol} / \mathrm{L})$, Hypocalcemia $(1,56 \mathrm{mmol} / \mathrm{L})$, Hypophosphoremia $(0,82 \mathrm{mmol} / \mathrm{L})$ and Hy- perparathyroidism $(65,8 \mathrm{pmol} / \mathrm{L})$. The radiography of the wrist displayed evidence of cupping, fraying, demineralization of the distal radial and ulnar metaphyses and metaphyseal widening. His heart condition has been immediately described as an higher left ventricular volume, mild mitral regurgitation and an ejection rate of $27,77 \%$ thus a severe reduction of global systolic function. A medical therapy with calcium and Vitamin D supplementation was started. After almost two weeks of therapy, as a control, an heart ultrasound was performed: the ventricular volume was nearly normal, no more mitral regurgitation and an ejection rate of $40 \%$. His heart failure was therefore attributed to severe vitamin D deficiency.

Conclusion: Vitamin D deficiency rickets can be life threatening especially with its uncommon presentations such as seizures, titanic crisis, heart failure and heart attack. Thus is important to recognize this condition and treat it with Vitamin D supplementation. In this case is described a great improvement of heart function just with Calcium and Vitamin D supplementation.

\section{EPO-040 Bone, Growth Plate and Mineral Metabolism \\ Novel mutation in GATA3 leads to complete phenotype of HDR syndrome in two familial cases}

Nathalie Burggraeve'; Corinne Magdelaine2; Sylvie Nivot-adamiak'; Marc De Kerdanet'; ; Anne Lienhardt ${ }^{3}$

'University hospital, Paediatric endocrinology unit, Rennes, France;

${ }^{2}$ University hospital, Biochemistry and genetic, Limoges, France;

${ }^{3}$ University hospital, Paediatric endocrinology unit, Limoges, France

Background: HDR syndrome is an autosomal dominant disorder consisting of hypoparathyroidism, sensorineural deafness and renal disease caused by mutation in the GATA3 gene located at chromosome 10p.

Objective and hypotheses: We report the clinical phenotype and molecular analysis of GATA3 in two french familial cases.

Results: Both patients have hypoparathyroidism, renal abnormalities, although they have not the same renal disease. The mother has sensorineural deafness. The daughter had normal hearing evaluations up to there. Recently, at 3 years old, she shows hearing impairment with delayed speech. We have found in both, a novel mutation of GATA3 in exon 6, which leads to a stop codon in the C- terminal tail of the protein ( $\mathrm{p}$ R353X), suppressing DNA binding. This mutation is heterozygous for both. The phenotype of our patients is compared with the phenotype of a Chinese family described in 2006 with a selective mutation in the same region.

Conclusions: These findings indicate a complete phenotype of HDR syndrome in these two patients with a selective mutation in the C-terminal tail of GATA3.

\section{EPO-041 Bone, Growth Plate and Mineral Metabolism Hypochondroplasia with hippocampal dysgenesis and neonatal onset of medial temporal lobe epilepsy \\ Tony Hulse $^{1}$; Jean-Pierre Lin²; Melita Irving ${ }^{3}$}

'Evelina Childrens Hospital, Department of Paediatric Endocrinology, London, United Kingdom; ' ${ }^{2}$ Evelina Childrens Hospital, Department of Paediatric Neurology, London, United Kingdom; ${ }^{3}$ Guys Hospital, Department of Clinical Genetics, London, United Kingdom

Background: Hypochondroplasia is an FGFR3-related type of skeletal dysplasia with variable severity resulting in mild short stature at one end of the spectrum to significant short stature and skeletal anomalies similar to achondroplasia at the other end. It is autosomal dominant disorder with a high new mutation rate and $72 \%$ of cases result from the mutation p.Asn540Lys. A number of other related FGFR3 related disorders have associated brain abnormalities but this has not been a marked feature of hypochondroplasia. Objective and hypotheses: We describe a $21 / 2$ yr old boy seen in the skeletal dysplasia clinic about whom there had been concerns antenatally because of short femur length and who developed seizures at 1 hour of age. EEGs in the neonatal period had revealed abnormalities in the left temporal lobe region. The seizures responded well to anti epilepsy drugs up to 19 months of age. Methods: At assessment at $21 / 2$ yrs he had disproportionate short stature [sitting height -0.5SDs, leg length -4.5SDs] and a skeletal survey showed features consistent with hypochondroplasia.

Results: Molecular analysis confirmed the pAsn540Lys FGFR3 mutation. 
Review of the head MRI undertaken at 3 months revealed bilateral abnormal orientation of the hippocampal heads.

Conclusions: While 4 reported cases of medial temporal lobe epilepsy [MTLE] related to hypochondroplasia have been previously described, it has not been described before in the neonatal period. It is probable that activating mutations in FGFR3 through receptor tyrosine kinase activity may have an effect on hippocampal development in addition to the well recognised effects on skeletal development seen in hypochondroplasia.

\section{EPO-042 Bone, Growth Plate and Mineral Metabolism}

\section{Can we trust PTH measurements?}

Elena Monti; Grazia Morandi; Rossella Gaudino; Francesco Doro;

Paolo Cavarzere; Evelina Maines; Giuseppina De Luca;

Franco Antoniazzi

University of Verona, Department of Life and Reproduction Sciences, Verona, Italy

Background: Primary hyperparathyroidism is a rare endocrine disorder in children. Plasma parathormone (PTH) can be detected by different methods, that vary in sensibility, specificity and reproducibility.

Case report: We report the case of a 3-year-old child with a history of recurrent lower limbs pain, absent during the night. Her physically examination was unremarkable: her height and her weight were 93,2 cm (M/+1 SD) and $13,3 \mathrm{Kg}(+1 \mathrm{SD})$, respectively, and she had no clinical signs of rickets. We assessed her bone mineral status, finding a very high plasma PTH value: $1493 \mathrm{pg} / \mathrm{ml}$ (normal range: 10-60 pg/ml, test LIAISON ${ }^{\circledR}$, N-TACT ${ }^{\circledR}$ PTH) with $25(\mathrm{OH})$ vitamin D level of $28,5 \mathrm{ng} / \mathrm{ml}$ and normal values of calcium, phosphate and ALP; urinary ions resulted within the normal range too. The bone ultrasound at her finger showed normal value of quantitative ultrasound parameters (AD-Sos and BTT z-score). The ultrasound of the neck revealed a solid nodule of 6 to $4 \mathrm{~mm}$ at the level of the left-lower pole of the thyroid, which was interpreted as an hypertrophic parathyroid. So we decided to perform whole body MIBI-scintigraphy, that was normal, and the analysis of the following genes: NHERF1, GNAS, PTHR1, PTH and MEN, all of them resulting negative, while RET gene analysis showed an heterozygous polymorphism in the exon 11, without any clinical relevance. We recommended the patient to take vitamin D supplementation, but we obtained only a partial and temporary decrease in PTH value $(246 \mathrm{pg} / \mathrm{ml})$. Anyway, the subsequent determination of plasma PTH, that was performed by another method (DiaSorin LIAISON 1-84), revealed a normal PTH value (17,6 pg/ml), despite the persistence of the parathyroid nodule.

Conclusions: Since the sensitivity of plasma PTH determination is poor, we recommend to repeat the analysis with different methods, if the results are inconsistent with the other laboratory, instrumental and clinical findings.

\section{EPO-043 Bone, Growth Plate and Mineral Metabolism}

\section{Primary hypoparathyroidism with calcification of basal ganglia: a case report}

Filiz Tutunculer; Emine Dilek; Sebile Kilavuz; Digdem Bezen

Medical School of Trakya University, Pediatric Endocrinology, Edirne, Turkey

Introduction: Primary hypoparathyroidism (PHP) is caused by a group of heterogeneous condition in which hypocalcemia and hyperphosphatemia occurs as a result of deficient parathyroid hormone (PTH) secretion. Although tetany and seizure are the most common symptoms in acute hypocalcemic state, intracranial calcification, subcapsular cataract and abnormal dentition may occur in chronic hypocalcemic condition.

Case: A 16 year-old male was transported to our hospital for the evaluation of his first tonic-clonic seizure and suspection of intracranial bleeding on his brain computerized tomography. His past history was unremarkable for muscle cramps or tetany. On admission, he was $178.5 \mathrm{~cm}$ (75-90 centile), weighed $56.4 \mathrm{~kg}$ (25-50 centile), body temperature was $36.7 \mathrm{C}$ and his blood pressure was $110 / 70 \mathrm{mmHg}$. He was consciousness and meningeal signs were absent. He had no facial dysmorphism, brachydactyly and subcutaneous calcification. Chvostek sign was positive but Trousseau sign was absent. Laboratory studies included: $5.7 \mathrm{mg} / \mathrm{dl}$ calcium (normal range, $8.6-10), 7,9 \mathrm{mg} / \mathrm{dl} \mathrm{phos-}$ phate (normal range, 2,3-4,7) and $3 \mathrm{pg} / \mathrm{ml}$ intact PTH (normal range, 11-65). Serum albumin level was $4,1 \mathrm{gr} / \mathrm{dl}$ (normal range $3,2-4,8$ ). Renal and liver function tests were within normal ranges. Brain computerized tomography demostrated symmetric calcification of basal ganglia. Slit lamp examination was normal. Based on the clinical and laboratory findings, he was diagnosed as PHP and was treated with parenteral calcium followed by oral calcium supplementation and calcitriol. After 1 week, his calcium level was $8,7 \mathrm{mg} / \mathrm{dl}$ and he did not experience another seizure.

Conclusions: We noticed that serum calcium level should be checked in every child who had a seizure because the seizure may be the first symptom caused by hypocalcemia.
EPO-044 Bone, Growth Plate and Mineral Metabolism

\section{A new association: normocalcemic} hyperparathyroidism and ectodermal dysplasia Nihal Hatipoglu'; Selim Kurtoglu' ; Mustafa Mumtaz Mazicioglu², Mehmet Boyraz

${ }^{1}$ Erciyes University, Medical Faculty, Pediatric Endocrinology, Kayseri, Turkey; ${ }^{2}$ Erciyes University, Medical Faculty, Family Medicine,

Kayseri, Turkey; ${ }^{3}$ Sisli Etfal Training and Research Hospital, Pediatric Endocrinology, Istanbul, Turkey

Backround: Normocalcemic hyperparathyroidism (NHP) is typically defined as normal serum calcium levels associated with inappropriately elevated parathyroid hormone (PTH) levels. NHP is an uncommon and especially a rare childhood pathologic finding. Ectodermal dysplasia (ED) is a rare and hereditary disorder that affects ectodermal structures.

Objective and hypotheses: The main clinical oral manifestations of ED include oligodontia.

Method: A 3-year-old girl presented with bowed leg deformity. She had dental anomaly but no rickets findings. Biochemical findings suggested NHP. Her parents were consanguineous. After 4 years old, she presented dental anomalies. She is still normocalcemic after 4 years. Her brothers ( 7 years old) and sisters (3 years old) had the same dental anomalies (Picture 1, 2, 3). They had no other ectodermal dysplasia findings. Results-Finding: Their biochemical findings suggested NHP (parathyroid hormone levels were high, calcium, phosphor, alkaline phosphatase, urine calcium creatinine and vitamin D levels were normal). Thyroid and parathyroid ultrasonography and scintigraphy, renal ultrasonography and bone scan findings were normal.

Conclusion: Both NHP and ED are rare disordors in childhood. It is known that ED is a herediter disorder but NHP isn't. These disorders probably might overlap within the same patients. However, we suggested that this stuation may be a new association.

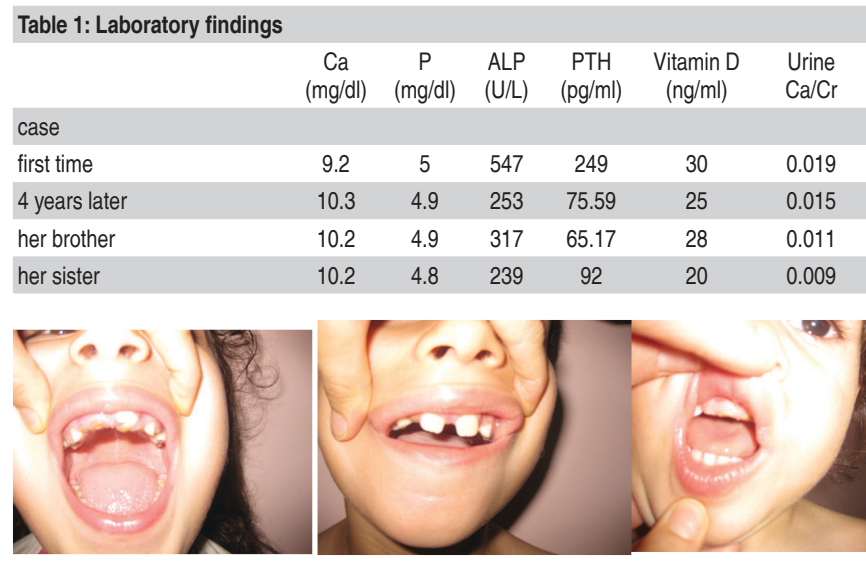

EPO-045 Bone, Growth Plate and Mineral Metabolism

Screening for IGF-1 deficiency in a laboratory data base using data mining methods

Francois Eyskens; Francois Eyskens

University Hospital/Queen Paola Chidlren's Hospital, Paediatrics, Antwerp, Belgium

Background: Defects in single genes in the GH-IGF-I axis can disrupt growth and decrease height velocity to below $4 \mathrm{~cm} /$ year and lead to an abnormal phenotype of short stature. Every child with short stature and growth failure who has low serum IGF-1 concentration with normal or elevated serum growth 
hormone, should be further evaluated for IGF-1 deficiency.

Methods: The immunoassay system used in the UZA Labo Hormonology for measuring serum IGF-1 is a RIA test commercialized by the firm DiaSource. The database of IGF-1 serum concentrations of the laboratory of the UZA was screened or scanned for values below the 0.1 percentile for age, and for gender after the age of 8 years while some girls will enter puberty at this age with concomitant rise in the serum IGF-1 concentration. Three populations of different age groups were studied: $0-4$ years; $4-8$ years; $8-10$ years. Serum IGF-1 concentrations below the cut-off values were further investigated. ICT method used in this study: The patients' database records was scanned (online or offline) against their respective age, gender, and IGF-1 laboratory results. This process was conducted fully automatically without visual inspection of the data. The output of the operation produced a shortlist with the ID's of those patients retained, in accordance with the criteria of the study. The files that needed further investigation were defined as:

- Patients with short stature and growth failure that had no or insufficient diagnostic evaluation in the past;

- Patients under growth hormone therapy, SGA and Turner syndrome excluded, who show inadequate or poor response to $\mathrm{GH}$ treatment as evaluated by the first year hight velocity below $1 \mathrm{SD}$.

Results: 28 cases who presented with persistently low serum IGF-1 were found:

-4 patients under growth hormone therapy who showed inadequate or poor response;

-6 patients with no or insufficient diagnostic evaluation;

-14 cases: different underlying causes: renal failure, syndromes, failure to thrive;

-4 cases: insufficient data were found in the files.

\section{EPO-046 Bone, Growth Plate and Mineral Metabolism}

\section{Characteristics and outcome of 7 patients with} pseudohypoparathyroidism

Lina Velásquez; María Albisu; Diego Yeste; Miguel Gussinye;

Silvia Grau; Laura Losada

Hospital Vall D’ Hebron, Paediatric endocrinology, Barcelona, Spain

Background: Pseudohypoparathyroidism is a heterogeneous disease characterized by resistance to the action of different hormones that activate Gscoupled receptors, mainly PTH. The two main subtypes of pseudohypoparathyroidism, Ia and $\mathrm{Ib}$, result from mutations or imprinting abnormalities in GNAS gene (encoding the Gsa protein). Predominant features of the disease are hypocalcemia and hyperphosphatemia. Subtype Ia is associated with Albright's hereditary osteodystrophy (AHO ) phenotype; in subtype Ib it can be absent or present in a mild form. GNAS mutations occur also in patients with pseudopseudohypoparathyroidism.

Objective: To Describe the characteristics, clinic course and genetic defect of seven patients diagnosed with Pseudohypoparathyroidism between January 1980 and december 2011.

Methods: Retrospective analysis of medical records.

Results: Patients are three men and four women, the mean age at diagnosis was 6.9 years (range: 1.5 to 14.5 ), three are already adults and four are under the age of 9 years. The diagnosis was established by the presence of high PTH levels in all patients with an mean of $420 \mathrm{pg} / \mathrm{ml}$ (range: 86 to 1056 ), associated to hypocalcemia plus hyperphosphatemia in 5 cases. The GNAS gene study was conducted in 4 patients, where inactivating mutations were found. 7 Patients showed AHO features, also, 6 patients showed high TSH levels with negative antithyroid antibodies during their illness. One woman displayed Hypogonadism manifested as secondary amenorrhea. GH stimulation tests showed a deficit in 3 tested patients. Final height of already adult patients are below $-2.5 \mathrm{SD}$, even for the one treated with $\mathrm{GH}$, who reached a final height of $-3.7 \mathrm{SD}$. Two of the studied patients are brothers, their mother shows a confirmed pseudopseudohypoparathyroidism.

Conclusions: PHP should be suspected in patients showing hyperphosphatemia and hypocalcemia; also, in those displaying low height plus peripheral hypothyroidism associated with some feature of AHO despite not having this biochemical alteration.

\section{EP0-047 Diabetes and Insulin}

Health related quality of life in the diabetic patients using the pedQoL diabetes module (Arabic version)

Magdy El-Ziny; Ashraf Elsharkawy; Amany Elhawary; Ahmed Zaky Mansoura University Children Hospital, Endocrinology and Diabetes Unit, Mansoura, Egypt

Background: Adolescents with T1DM as a group display the worst glycemic control compared with other age groups. Health-related quality of life (HRQoL) is typically defined as patients' appraisal of their current level of functioning; psychological, physical, and social health; and satisfaction compared to what they perceive to be ideal. The Pediatric Quality of Life Inventory (PedsQL) is a modular instrument designed to measure health-related quality of life (HRQOL) in children and adolescents aged 2-18 years.

Objective and hypotheses: To evaluate the health related quality of life in the diabetic patients in our locality (Mansoura, Egypt), we used the pedQoL diabetes module produced by Varni et al., 2003.

Methods: 45 patients recruited consequently from the patients following with the out patient clinic of Mansoura university child hospital, endocrinology and diabetes department. At the routine diabetes visit, we conducted a structured, $5-10 \mathrm{~min}$, joint interview (patients and parents) to gather demographic information and assess diabetes management tasks during the preceding month. Each child and a parent (45 patients and their parents) also completed questionnaires by his own which included diabetes related problems (11 items), treatment related problems (11 items) and psychosocial problems (6 items). Results: Total quality of life, physical disease problems, treatment problems, and psychosocial problems were examined. As regard the physical disease problems reported by the patients $4.4 \%$ never reported a problem, $40 \%$ almost never had a problem, $53 \%$ some times had a problem, and $2.2 \%$ often had a problem. As regard the treatment problems reported by the patients $17.8 \%$ never reported a problem, $60 \%$ almost never had a problem, $22.2 \%$ some times had a problem, and none often had a problem.

Conclusions: Most of our patients and their families complain from physical disease problem than treatment problem itself.

\section{EPO-048 Diabetes and Insulin \\ Long-term changes of visual evoked potentials in children with insulin-dependent diabetes mellitus}

Heon-Seok Han'; ; Jaehong Yu

${ }^{1}$ Chungbuk National University Hospital, Pediatrics, Cheongju,

Republic of Korea; ${ }^{2} J o y$ Children Hospital, Pediatrics, Daejeon

Republic of Korea

Background: Early detection of neurologic alteration, either in central or peripheral, is an important task in the follow-up of diabetic patients. While the involvement of peripheral and autonomic nervous system determines a large spectrum of clinical manifestations, CNS involvement is usually clinically silent.

Objective and hypotheses: To investigate the changes of central nerve conduction in children with insulin-dependent diabetes mellitus prospectively using the visual evoked potentials (VEP) and to know how those results were related to clinical risk factors and parameters of peripheral nerve conduction studies (NCS).

Methods: A total of 76 patients (29 males and 47 females) aged 5-26 years (mean 14.4 \pm 4.8 ) with IDDM underwent visual evoked potentials and bilateral NCS of median, ulnar, posterior tibial, peroneal, and sural nerves annually for 5 years. Control group was made up of healthy 52 children.

Results: Out of 76 patients, 25 patients completed annual studies for 5 years. Significant positive correlations were found between the latency of P100 wave in the VEP and the level of serum glycosylated hemoglobin. Latency and amplitude values of P100 waves decreased progressively in children with IDDM as the duration of the disease increased. The values of latency and amplitude were inversely related with the age of patients. The values of latency of P100 were not related with parameters of NCS. However, some of parameters of NCS were weakly related with the amplitudes of 100 .

Conclusions: Poor glycemic control proved to be an important risk factor over 5 years as related to the development of subclinical central neural pathway abnormality. VEP could be considered as a valid noninvasive tool for detecting an early diabetic central conduction abnormality such as retinopathy or optic neuropathy. 


\section{EP0-049 Diabetes and Insulin}

HNF1A mutation in a patient with diabetes, hydronephrosis and renal stone

Ozlem Engiz'; Yildiz Bilge Dallar ${ }^{2}$; Sian Ellard'; Emine Gocer ${ }^{2}$; Emine Potur ${ }^{2}$

${ }^{1}$ Ankara Training and Research Hospital, Pediatric Endocrinology,

Ankara, Turkey; ${ }^{2}$ Ankara Training and Research Hospital, Pediatrics,

Ankara, Turkey; ${ }^{3}$ Exeter University, Molecular Genetics, Exeter, United Kingdom

Background: Heterozygous mutations of glucokinase and hepatocyte nuclear factor-1 alpha (HNF1A) genes are the most common cause of MODY. Developmental anomalies such as renal agenesis, hypoplastic kidney and renal cysts have been reported in patients with HNF1A mutations but hydronephrosis and renal stone with this mutation have not been reported previously.

Results: A 13-year-old male was referred to Ankara Training and Research Hospital with hyperglycemia. His random blood glucose was $489 \mathrm{mg} / \mathrm{dl}$. His urine had traces of glucose with no ketones. He had no history of polyurea, polydipsia, weight loss or bed wetting. He was born at term with a birthweight of $1400 \mathrm{~g}$. His 36-year-old father was diagnosed with type 1 diabetes at the age of 32. He had been on insulin treatment since then. The father's mother was diagnosed with type 2 diabetes at the age of 54 and she had been using glipizid and metformin for the last 4 years. The mother and none of her family had diabetes.

On admission, his HbAlc was 9.0\% (normal 4-6). Fasting blood glucose was $170 \mathrm{mg} / \mathrm{dl}$, fasting insulin $5.92 \mathrm{uIU} / \mathrm{mL}$, fasting C peptide: $0,60 \mathrm{nmol} / \mathrm{L}$, postprandial blood glucose: $266 \mathrm{mg} / \mathrm{dl}$, postprandial insulin: $6,86 \mathrm{uIU} / \mathrm{mL}$, postprandial C peptide: $2.28 \mathrm{nmol} / \mathrm{L}(0,28-2)$. Islet cell autoantibodies (ICA) and glutamic acid decarboxylase autoantibodies (GADA) were negative. Renal ultrasonography demonstrated grade 3 hydronephrosis and renal stone of the right kidney. Direct DNA sequencing of exons 1 to 10 and flanking intronic regions of the HNF1A gene was done at Peninsula Medical School, University of Exeter. The analysis showed that the child was heterozygous for a known missense mutation (c.448A $>\mathrm{G}$ ) in exon 2 of the HNF1A gene (p.Lys150Glu (p.K150E)).

Conclusions: HNF1A mutations should be remembered in patients with monogenic diabetes, hydronephrosis and renal stone as well as renal developmental anomalies.

\section{EPO-050 Diabetes and Insulin}

\section{Assessment of efficiency insulin pump therapy in school-age children with type 1 diabetes} Irina Nikitina; Liliya Ditkovskaya; Julia Skorodok; Julia Demchuk; Anastasia Todieva

Federal State Institution "Federal Center of Heart, Blood and Endocrinology named after V.A. Almazov", Pediatric endocrinology scientific laboratory, Saint-Petersburg, Russian Federation

Background: Insulin pump therapy is at present the best way to imitate the physiological insulin profile. But unequivocal evidence for the benefit of multiple daily injections (MDI), the analogs and continuous subcutaneous insulin infusion (CSII) treatment in children is lacking.

Objective and hypotheses: The aim of the study was to estimate the influence of insulin pump therapy on metabolic control and quality of life of children with type 1 diabetes.

Methods: We examinated 37 patients in average age 12,1+3,5 years with type 1 diabetes twice: in MDI and more than 1 year after translation on CSII. CSII has been compared to MDI in metabolic control and quality of life by protocol PedsQL (Type 1 Diabetes Module, Version 3.2).

Results and Conclusion: In CSII daily variability of glycemia, frequency of ketoacidosis and hypoglycemia was decreased. There was mild decrease of $\mathrm{HbA} 1 \mathrm{c}-$ from $8,8 \pm 1,6 \%$ to $7,8 \pm 1,5 \%(\mathrm{p}>0,05)$. In this study comparing MDI vs. CSII, diabetes treatment satisfaction was higher with CSII. Presence of improvement of self-feeling, reduction of problems with treatment and worry connected with disease were observed with CSII. However, the rates of social communication did not change.

\section{EPO-051 Diabetes and Insulin}

Clinical variability in the use between glulisine vs. aspart/humalog insulin in DM1 children with insulin pump

Ignacio Diez-Lopez; Ainhoa Sarasua; Blazquez Lorente

University Hospital of Alava, Peadiatric Endocrinology Unit, Vitoria, Spain

Introduction: The chemical composition of the different analogues gives them specific pharmacokinetic-dynamic characteristics. The absence of zinc in glulisine, allows it a lower crystallization, a possible advantage in catheter devices. There are no previous studies about this peculiarity in children DM1 patients with insulin pump in our country. From October to December 2011 there is an unexpected event interrupts pharmacist glulisine distribution worldwide. All patients during this time users should go to another fast insulin analogue.

Objective: To evaluate the impact of this event, and therefore the change of insulin in the metabolic control and quality of life of children with CSII.

Methods: DM type 1a children with at least 36 months of evolution from theis debut and 12 months of using pump with insulin glulisine. Study variables $\mathrm{HbA} 1 \mathrm{c}$ (DCA) in fasting blood glucose (FBG), postprandial (PPG), insulin requirements (IR), day use catheter (DC) and overall satisfaction survey (1-10) (SS). Forced to change aspart / humalog and reassessment in January 2012.This is a comparative study. SPSS 17.0., Nonparametric paired samples $\mathrm{n}<30$.

Results: 5 children ( 2 males), mean age 11.5 years [8-13]. With glulisine and before the change; variables: HbA1c (DCA): 7.6\% [6.8-8.2], FBG $127 \mathrm{mg}$ / dl [65-185], PPG $149 \mathrm{mg} / \mathrm{dl}$ [98-195], OR: $0.88 \mathrm{IU} / \mathrm{kg} /$ day [0.72-1.18], DC: 4.3 days [3.5-5] and SS 8.5 points [7-10]. After period without glulisine and aspart / humalog used; variables: HbA1c (DCA): 8.2\% [7.4-9.1] p differences: $0.0295 \%$ CI [0.4 to 0.8], FBG $148 \mathrm{mg} / \mathrm{dL}$ [102-190] differences p: $0.0195 \%$ [42-68], PPG $175 \mathrm{mg} / \mathrm{dL}$ [141-245] p: 0.04 95\% [32-76], IR: 1.02 $\mathrm{IU} / \mathrm{kg} /$ day [0.85-1.31] p differences: $0.0195 \% \mathrm{CI}$ [0.12 to 0.34$]$, DC: 2.6 days [ 2-3.5] p: $0.00195 \%$ CI [1.5-2.5], and SS 6 points [5-7].

Conclusion: Although the study power could be low for $\mathrm{n}$; the results point to the potential clinical and economic advantages that may result from use of glulisine in these patients. We recommend this type of insulin in children DMtipo1 CSII.

\section{EPO-052 Diabetes and Insulin \\ Severe hypercalcaemia secondary to severe, prolonged metabolic acidosis in a patient with DKA}

T Makaya' ; P ArundeP; C Bevan ${ }^{3}$; NP Wright

'Sheffield Children's Hospital, Paediatric Endocrinology And Diabetes, Sheffield, United Kingdom; ${ }^{2}$ Sheffield Children's Hospital, Department of Metabolic Bone Disease, Sheffield, United Kingdom; ${ }^{3}$ Sheffield Children's Hospital, Department of Critical Care, Sheffield, United Kingdom; ${ }^{4}$ Sheffield Children's Hospital, Department of Paediatric Endocrinology, Sheffield, United Kingdom

Background: Children presenting with diabetic ketoacidosis(DKA) as an initial presentation of diabetes mellitus are often unwell, with associated increases in mortality and morbidity. While electrolyte imbalances such as hypokalaemia and hypophosphataemia are well recognised, hypercalcaemia is less well documented.

Objective and hypotheses: Case presentation: A previously healthy 12-year old boy presented to hospital with a history suggestive of new onset diabetes. Initial bloods indicated DKA: pH 6.84, BE -28.9 and plasma glucose $30.4 \mathrm{mmol} / \mathrm{L}$. Clinically he was severely dehydrated (estimated $8 \%$ ). Despite standard management according to national guidelines he developed a reduced GCS, presumed secondary to cerebral oedema, requiring intubation and ventilation.

Methods: Progress: He remained severely acidotic, which was initially secondary to keto- and lactic-acidosis but was then propagated by hyperchloraemia. Over the next few hours he gradually developed acute severe hypercalcaemia, with maximum corrected calcium of $3.75 \mathrm{mmol} / \mathrm{L}$. Possible causes for hypercalcaemia including hyperparathyroidism, malignancy, and thyrotoxicosis were ruled out. He developed mild-moderate renal failure (maximum creatinine $269 \mathrm{mmol} / \mathrm{L}$ ). He was treated cautiously with rehydration as part of a neuro-protective strategy and latterly treated with frusemide infusion and hydrocortisone. Calcium levels and renal function normalised within a week. 
Results:

\begin{tabular}{|c|c|c|c|c|c|c|c|c|c|c|}
\hline & $\begin{array}{c}\text { corrected } \\
\text { calcium } \\
\mathrm{mmol} / \mathrm{L}\end{array}$ & $\begin{array}{c}\mathrm{PO4+} \\
\mathrm{mmol} / \mathrm{L}\end{array}$ & $\underset{\mathrm{Mg} 2+}{\mathrm{mmol} / \mathrm{L}}$ & $\begin{array}{l}\text { PTH } \\
\mathrm{ng} / \mathrm{L}\end{array}$ & $\begin{array}{c}\text { Total } \\
\text { Vitamin D } \\
\text { nmol/L }\end{array}$ & $\begin{array}{l}\text { Urine } \\
\text { cal/creat } \\
\text { ratio }\end{array}$ & $\underset{\mathrm{mmol} / \mathrm{L}}{\mathrm{Na} 2+}$ & $\underset{\mathrm{mmol} / \mathrm{L}}{\mathrm{Cl}}$ & $\mathrm{pH}$ & BE \\
\hline DO & 2.57 & & & & & & & & 6.84 & -28.9 \\
\hline D2 & 3.75 & 0.36 & 1.01 & 8.3 & 22 & 0.17 & 161 & 137 & 7.08 & -21.2 \\
\hline D4 & 3.72 & 0.62 & 0.92 & & & & 167 & 143 & 7.32 & -14.4 \\
\hline D6 & 2.76 & 0.91 & & & & & 148 & 121 & 7.38 & -4.7 \\
\hline D8 & 2.56 & 1.23 & & & & & 142 & 108 & & \\
\hline D10 & 2.46 & 1.56 & & 45.4 & & & & 100 & & \\
\hline
\end{tabular}

Conclusions: Potassium and phosphate disturbances are common in DKA, however significant abnormalities in calcium haemostasis are less common. Severe hypercalcaemia in DKA is likely due to diminished bone formation mediated in part by metabolic acidosis, paired with increased bone resorption due to severe insulin deficiency and metabolic acidosis. We suggest that calcium concentrations are checked routinely in all DKA patients.

\section{EPO-053 Diabetes and Insulin}

\section{Clinical presentation of diabetes mellitus in youngsters living in Cali Colombia from two paediatric endocrinology consults}

Audrey Mary Matallana'; Liliana Mejia de Beldjenna²;

Francisco Javier Bonilla-Escobar'; Maria Lucia Valderrama ${ }^{3}$

${ }^{1}$ Universidad del Valle, Valle, Cali, Colombia; ${ }^{2}$ Clinica Valle del LILI,

Fundación Infantil Club Noel, Universidad Libre, Valle, Cali, Colombia;

${ }^{3}$ Universidad Libre, Valle, Cali, Colombia

Background: Clinical presentation of diabetes mellitus in youngsters living in Cali is not known.

Objectives and hypothesis: To characterize the patients with diabetes mellitus attending in endocrine clinics in Cali Colombia between 1995-2011.

Methods: Data were obtained from patients with diabetes mellitus to evaluate the characteristics and clinical presentation by univaried and multivaried analysis. We obtained contingency tables and we applied a chi 2 and Fisher's test to confirm the hypothesis, $95 \%$ confidence limits and a $\mathrm{p}$ value of $<0.05$ for statistical significance.

Results: We analized 118 patients .The year 2009 and the month May were the highest with $13,6 \%(16)$ cases . No differences between males and females .Family history of diabetes was present in $44.9 \%(53)$. Onset with Ketoacidosis in 41.3(49).We found a lineal correlation between age and glycated hemoglobin of an increase of $0.2 \%$ in $\mathrm{HbA} 1 \mathrm{C}$ for each year of increase in age in $13 \%$ of the patients.

Conclusions: We proposed two hypothesis : 1-At onset of Diabetes Age and glycated hemoglobin correlate, 2-DM onset in Cali has a seasonal association.Further studies are necessary to clarify these hypothesis.

\section{EPO-054 Diabetes and Insulin \\ Clinical characteristics and laboratory findings of children and adolescents with diabetes mellitus \\ Se Young $\mathrm{Kim}^{1}$; Eun Young $\mathrm{Kim}^{2}$; Kyung $\mathrm{Hee} \mathrm{Y}^{3}$ \\ ${ }^{1}$ Bundang Jesaeng General Hospital, DMC, Pediatric Endocrinology, \\ Pediatrics, Seongnam, Republic of Korea; ${ }^{2}$ School of Medicine, \\ Chosun University, Pediatrics, Gwangju, Republic of Korea; \\ ${ }^{3}$ Wonkwang University Hospital Sanbon Medical Center, Pediatrics, \\ Gunpo, Republic of Korea}

Background: Objective and hypotheses: The incidence of childhood type 2 diabetes mellitus (DM) is increasing worldwide in parallel with an increasing prevalence of childhood obesity. We investigated the type of diabetes and the clinical characteristics in the newly diagnosed diabetic children.

Methods: Retrospective analysis of clinical characteristics was done in 125 newly diagnosed diabetic children and adolescents under 18 years of age at University Hospital in Korea from March 2003 to December 2010.

Results: Children diagnosed with type 1 diabetes were 100 out of $125(80 \%)$ and 25 out of $125(20 \%)$ were type 2 diabetes. Mean age of onset was 9.26 \pm 0.99 years and there was no seasonal variation of incidence. $32 \%$ of children with type 1 diabetes presented initially with diabetic ketoacidosis. Mean body mass index (BMI) was $16.8 \pm 3.8 \mathrm{~kg} / \mathrm{m} 2$, mean blood glucose level was $457.6 \pm 212.5 \mathrm{mg} / \mathrm{dL}$ and mean glycated hemoglobin (HbA1c) level was $12.1 \pm 2.28 \%$. Positive result was revealed in $52 \%$ of the subjects with type 1 diabetes for antibodies to glutamic acid decarboxylase (GAD), 3\% for isletcell antibodies (ICA), 25\% for insulin autoantibodies (IAA) and 63\% showed positive results for at least one of these autoantibodies. 25 patients $(20 \%)$ were diagnosed with type 2 diabetes. Mean BMI was $28.3 \pm 8.7 \mathrm{~kg} / \mathrm{m} 2$, mean blood glucose level was $217.7 \pm 105.5 \mathrm{mg} / \mathrm{dL}$ and mean $\mathrm{HbA} \mathrm{cc}$ concentration was $9.0 \pm 2.9 \% .52 \%$ of the subjects diagnosed with type 2 diabetes had a family history of diabetes and $80 \%$ were either overweight or obese.

Conclusions: Although still not as common as type 1 diabetes among children, type 2 diabetes mellitus increasingly has been seen in children. Routine medical screening in obese children and adolescents or ones with other risk factors of type 2 diabetes should be emphasized to make early diagnosis and start management of type 2 diabetes to improve long-term outcomes.

\section{EPO-055 Diabetes and Insulin \\ Is there a gap between self versus professionals' judgment of metabolic control in type 1 diabetic children and adolescents Andreas Bieri; Monika Oser-Meier; Marco Janner; Kathrin E. Pipczynski-Suter; Primus E. Mullis; Christa E. Flück University Children's Hospital, Inselspital, Paediatric Endocrinology, Diabetology \& Metabolism, Bern, Switzerland}

Background: Morbidity and mortality in T1DM depends largely on good metabolic control. Metabolic control is usually assessed by professionals with 3-monthly measurements of HbA1c levels and counseling is delivered accordingly. By contrast, patients' self-judgment of glycemic control depends mainly on daily blood glucose monitoring. Little is known about the correlation between patients' and professionals' judgment of metabolic control in T1DM.

Objective: To assess whether there is a gap between patients' self-judgment and objective assessment (HbA1c) of metabolic control in T1DM children and adolescents and what factors may be associated.

Methods: Cross-sectional, retrospective and prospective cohort study with T1DM patients aged 8 to 18 years $(n=91)$ seen at our outpatient clinic from $04 / 2011$ to $09 / 2011$. We collected patients' data such as HbAlc levels, treatment modality, blood glucose monitoring and socioeconomic status. Patients were evaluated using a questionnaire containing parameters of self-judgment of metabolic control. These data were analyzed and compared with professionals' judgment of metabolic control.

Results: A total of 91 patients $(53 \mathrm{~m}, 38 \mathrm{f})$ were included. $9.9 \%(\mathrm{n}=9)$ were conventionally treated, $64.8 \%(\mathrm{n}=59)$ on functional insulin treatment and $25.3 \%(\mathrm{n}=23)$ treated with an insulin pump. Mean age was 13.2 years (range 8.2-17.8). Mean interval since initial diagnosis was 6.1 years $(1.1-15.8)$. Mean HbA1c level was $8.0 \%$ (6.1-10.9). Self-judgment of metabolic control, remembering $\mathrm{HbA} 1 \mathrm{c}$ level of last control and target level of $\mathrm{HbA} 1 \mathrm{c}$ correlated with actual $\mathrm{HbA} 1 \mathrm{c}$ levels and with diabetes duration. We found no clear correlation with age, blood glucose home monitoring and socioeconomic status. Conclusions: Well controlled T1DM patients seem to have better self-judgment concerning their diabetes control. Poorly controlled patients rather lack proper judgment of their diabetes control. Therefore professionals should pay special attention in teaching patients with less controlled T1DM about good glycemic control.

\section{EP0-056 Diabetes and Insulin}

\section{A rare case of ketoacidosis: case report}

Joana Rebelo; Cíntia Castro Correia; Manuel Fontoura

Centro Hospitalar São João, EPE, Pediatric Endocrinology Unit, Porto, Portugal

Background: Persistent hyperinsulinemic hypoglycemia of infancy (PHHI) is characterized by inappropriate elevated secretion of insulin in the presence of serious hypoglycemia. Diazoxide is frequently used as a treatment and is usually well tolerated, although some rare and serious adverse effects have been reported.

Objective and hypothesis: Presentation of a case report regarding adverse effects resulting from accidental diazoxide overdosage in a patient with PHHI. Method: We present the case of a six month-old infant admitted with seizures in the context of serious non-ketotic hypoglycemia. PHHI was diagnosed 
and he was started on diazoxide therapy $(5,1 \mathrm{mg} / \mathrm{kg} / \mathrm{day})$, with subsequent normalization of glycemia, which he maintained for the next two months. At eight month-old he was readmitted with fever, vomit and hyperglycemia. Ketonuria, compensated ketoacetic acidosis and neutropenia (500/uL) were observed. Over the next $48 \mathrm{~h}$ he developed irritability and congestive heart failure signs; pulmonary hypertension was diagnosed by echocardiography. Results and conclusion: At this point, a more extensive anamnesis revealed the clinical picture's etiology.

\section{EPO-057 Diabetes and Insulin}

\section{KCNJ11 gene mutations in a family with permanent neonatal diabetes}

Farzaneh Abbasi; Saba Sadaf; Ebrahim-Habibi Azadeh;

Sayahpour Forough A; M Amoli Mahsa

Endocrinology and Metabolism Research Center), Tehran University

of Medical Sciences, Tehran, Iran., endocrinology, tehran, Islamic

Republic of Iran

Background: Activating mutations of KCNJ11 which encodes Kir6.2 (beta-cell KATP channel subunit) have been associated with neonatal diabetes (NDM) in different studies. Treatment with sulfonylurea shows successful long term results with increased insulin secretion and improved glycemic control in patients carrying these mutations. Exploring genetic cause of diabetes before the age of 6 months has been proposed as important issue in identification of monogenic forms of diabetes which might be critical in their therapeutic management as a consequence.

Methods: Mutation screening for KCNJ11 gene in 3 members of a family whom previously were diagnosed as type 1 diabetes (T1DM) and were under insulin treatment has been carried out using PCR amplification followed by direct sequencing.

Results: Our results revealed the presence of a heterozygous missense mutations c. $1247 \mathrm{G} / \mathrm{A}(\mathrm{E} 227 \mathrm{~K})$ and several polymorphisms c.635 A/G (K23E), c. $1577 \mathrm{G} / \mathrm{A}$ (V337I), c.1369 C/G and c.1711G/A in all three patients. The effect of E227K mutation was also examined on a homology model of Kir6.2. Patients' treatment has been successfully transferred from insulin injection to oral sulfonylurea with a good glycemic control.

Conclusion: We have found an association between E227K mutation and PNDM in 3 members of a family which resulted in transition of insulin therapy to oral sulfonylurea. This mutation has been previously reported in patient with TNDM. Various phenotypic manifestations might be due to difference in genetic background or environmental factors which requires further investigations.

\section{EPO-058 Diabetes and Insulin}

\section{Diabetes program at schools in Turkey}

Sukru Hatun'; Hulya Gunoz ${ }^{2}$; Nesibe Andiran ${ }^{3}$; Peyami Cinaz

${ }^{1}$ Kocaeli university, school of medicine, Pediatric endocrinology and diabetes, Kocaeli, Turkey; ${ }^{2}$ Istanbul university, school of medicine, Pediatric endocrinology and diabetes, Istanbul, Turkey; ${ }^{3}$ Kecioren training and research hospital, Pediatric endocrinology and diabetes, Ankara, Turkey; ${ }^{4}$ Gazi University, school of medicine, Pediatric endocrinology and diabetes, Ankara, Turkey

Background: There are approximately 20.000 diabetic children in Turkey, who are mostly at school ages and it is possible to solve the problems by means of a comprehensive program. Diabetes Program at School is developed by the Turkish Society of Pediatric Endocrinology and Diabetes in cooperation with the Ministry of National Education and Ministry of Health. The program has three objectives: a)Raising awareness about Type 1 Diabetes by means of teachers, b)Strengthening the diabetic child's care at school ages, c) Generating a healthy nutrition attitude among school children and raising awareness about obesity.

Methods: An awareness poster under the title "Is my child a diabetes patient?" is now on the walls of schools across Turkey. It has reached to approximately 650.000 teachers and 16 million school children at primary and middle schools. Access to a brochure on diabetes in childhood, a guide on diabetic child care at school and a presentation on the training of teachers has been provided on related web pages. Pediatric Endocrinology Clinics across the country began to send a standardized letter on the diabetic child's care at school to diabetic children and their teachers. School children (7.500.619 children) and teachers (583.182 teacher) have jointed the school trainings to be organized within the scope of Diabetes Program at School and two films with a total duration of 24 minutes runed at 24836 schools, where the issues related to Diabetes and obesity among children are explained. For this reason, a training platform is generated on the website www.okuldadiyabet.org to access educational material.

Conclusions: The signs and symptoms of diabetes in children were told the teachers, students and community, strongly. The problems faced by diabetic children in school were pointed out. Permanent education materials about diabetes and obesity were created for the teachers and students.

\section{EPO-059 Diabetes and Insulin \\ Pathophysiology of critical illness hyperglycemia in children and effectiveness of a standardized treatment protocol}

Bulent Hacihamdioglu'; Tanil Kendirli'; Gonul Ocal'; Zeynep Siklar';

Senay Erdeve Savas'; Erdal Ince ${ }^{3}$; Merih Berberoglu'

${ }^{1}$ Ankara University Faculty of Medicine, Departments of Pediatric

Endocrinology, Ankara, Turkey; ${ }^{2}$ Ankara University Faculty of Medicine, Departments of Pediatric Critical Care, Ankara, Turkey; ${ }^{3}$ Ankara

University Faculty of Medicine, Critical Care, Ankara, Turkey

Background: Cause of hyperglycemia in critical ill non-diabetic children is different from adults, multifactorial and not completely understood.

Objective and hypotheses: The objective of the present study is investigate the pathogenesis of critical illness hyperglycemia $(\mathrm{CIH})$ and evaluated result of our insulin standardized treatment protocol in critically ill children.

Methods: Critically ill children with blood glucose (BG) level $>150 \mathrm{mg} / \mathrm{dL}$ $(8.3 \mathrm{mmol} / \mathrm{L})$ were enrolled. Insulin sensitivity and beta cell function on hyperglycemic and euglycemic period were investigated by using BG/insulin, BG/C-peptide and by homeostasis model assessment (HOMA) [beta-cell function $(\mathrm{HOMA}-\% \mathrm{~B}$ ) and insulin sensitivity $(\mathrm{HOMA}-\% \mathrm{~S})]$. Insulin was administered when BG over the $180 \mathrm{mg} / \mathrm{dL}(10 \mathrm{mmol} / \mathrm{L})$.

Results: Totally 232 patients followed to our pediatric intensive care unit (PICU) during this study period and $40(\% 17.2)$ of them diagnosed as $\mathrm{CIH}$. $\mathrm{BG} /$ insulin and BG/C-peptide ratio were significantly higher in the hyperglycemic period. In hyperglycemic period, $67.5 \%$ of the patients had beta cell dysfunction and $35 \%$ of them had insulin resistance according to the HOMA$\mathrm{B}$ and $\mathrm{S}$ scores. beta cell dysfunction significantly higher in hyperglycemic period $(\mathrm{p}<0.001)$

During the study period 192 patients without $\mathrm{CIH}$ and 17 of them (\%8.8) were died. However in CIH group $10(\% 25)$ patients were died $(p=0.006)$. Twenty nine of 40 patients were received insulin therapy and no patient developed hypoglycemia during the insulin therapy.

Conclusions: Beta cell dysfunction may be a major role in pathophysiology of $\mathrm{CIH}$ in children rather than insuline resistance which is seen in adults. Hypoglycemia can be avoided if tight glycemic control is not aimed in $\mathrm{CIH}$ treatment.

\section{EPO-060 Diabetes and Insulin}

\section{Lipoatrophic diabetes - a case report}

Maia Konstantinova'; Radka Savova ${ }^{1}$; Margarita Arshinkova'; Iva Stoeva ${ }^{2}$; Emilia Panteleeva ${ }^{3}$; Sian Ellard ${ }^{4} ; K_{\text {Colclough }}^{4}$

'University Pediatric Hospital, Endocrinology, diabetes and genetics, Sofia, Bulgaria; ${ }^{2}$ University Pediatric Hospital, Sector screening and functional endocrine diagnosticsEndocrinology, diabetes and genetics, Sofia, Bulgaria; ${ }^{3}$ University Pediatric Hospital, Gastroenterology, Sofia, Bulgaria; ${ }^{4}$ Royal Devon and Exeter NHS Hosp., Molecular genetics, London, United Kingdom

Aim: To present a patient with Lipoatrophic diabetes, diagnosed in July 2010. History of the disease: A 17 years old girl born after uneventful pregnancy and weight $3300 \mathrm{~g}$. with normal development, menarche at the age of 13 years. Her mother is obese and has type 2 diabetes, treated with metformin for 10 years. At the age of 4 years lipoatrophy of both buttocks noticed, thought to be caused by Paniculitis Weber-Christian/biopsy/ and therapy with steroids was implemented for 8 years. At the age of 12 years acanthosis nigricans appeared in parallel with the extension of the atrophic changes of the upper and lower extremities.

At the age of 15 years because of secondary amenorrhea further examinations /OGTT/ evidenced diabetes / blood glucose 18.2 and $29.6 \mathrm{mmol} / \mathrm{l}$ with hyperinsulinemia /156.3 mU/l and $187.3 \mathrm{mU} / 1 / 0$ ' and 120' resp. 
Results: The proposed diagnosis of Lipoatrophic diabetes was confirmed: $\mathrm{HbA} 1 \mathrm{c}-10.06 \%$ with negative autoantibodies /anti - GAD 65, IA-2 and IAA/; HLA- DRB $1 * 03, * 14$; DQB $1 * 02 * 05$; elevation of the hepatic enzymes /ASAT 141U/1, ALAT 210U/1, GGT 67U/1/; Hypertriglyceridemia /3.1 $\mathrm{mmol} / \mathrm{l} . /$ total cholesterol $5.31 \mathrm{mmol} / \mathrm{l}$, HDL-chol. $-0.68 \mathrm{mmol} / \mathrm{l} /$. FSH, LH, E2,Testosterone, Prolactin, and 17-OHP and Leptin were found to be in the normal ranges.

Ultrasonography: Hepatic steatosis and polycystic ovaries. Sequencing of exones 1-12 of the LMNA gene failed to detect a change from the normal sequence which cannot exclude the diagnosis since mutations of other genes /e.g. PPARG/ may cause it. Therapy with metformin and diet were started. HbA1c /November 2011/ - 6.6\%, a tendency for improvement of the lipids and hepatic enzymes. The menstrual cycle has been restored since Jan. 2011. The severity of the acanthosis nigricans has not changed. This rare form of diabetes is difficult for treatment, though the initial results are satisfying especially concerning glycemic control. However the lypoatrophic features remain unchanged. Therapy with leptin has been successful in some of the severe forms of lypodystrophy.

\section{EP0-061 Diabetes and Insulin}

\section{Permanent neonatal diabetes due to a novel insulin signal peptide mutation}

Suhaimi Hussain'; Johari Mohd Ali; Kha Chin Long'; Fatimah Harun ${ }^{3}$ ${ }^{1}$ Universiti Sains Malaysia, Department of Paediatrics, School of Medical Scienes, Kubang Kerian, Kelantan, Malaysia; ${ }^{2}$ University of Malaya, Department of Molecular Medicine, Kuala Lumpur, Malaysia; ${ }^{3}$ University of Malaya, Department of Paediatrics, Kuala Lumpur, Malaysia

Background: Monogenic form of diabetes is rare, but the commonest form is maturity-onset diabetes of the young (MODY). Neonatal diabetes is the other form of monogenic diabetes that is usually defined as overt diabetes diagnosed during the first 6 months of life. Permanent Neonatal Diabetes Mellitus (PNDM) due to insulin (INS) gene mutations was first described in 2007. INS mutations can cause a spectrum of clinical conditions such as type $1 \mathrm{~b}$ diabetes, MODY, early onset type 2 diabetes and neonatal diabetes. Some mutations thus far reported cause proinsulin misfolding or its retention in the endoplasmic reticulum (ER), leading to ER-stress induced $\beta$-cells apoptosis. Objective and hypotheses: The proband is a suggestive PNDM case based on these clinical findings. From Day 2 of life, she showed persistently high blood sugar ranging from $15.0-30.0 \mathrm{mmol} / \mathrm{L}$ which requires supplemental insulin (IV) at $0.1 \mathrm{U} / \mathrm{kg} /$ hour. There was no evidence of DKA or other illnesses. The C-peptide value was low $(<165 \mathrm{pmol} / \mathrm{L})$ and GAD antibody was negative. Subcutaneous insulatard was started to replace insulin infusion from Day 10. At her current age of 4 years 2 months, she still requires insulatard at about $0.5-0.6 \mathrm{U} / \mathrm{kg} /$ day. The median HbA1c ranged from 7.4-14.0. KCNJ11 and INS are among the genes implicated in early age diabetes/PNDM, thus mutation screening for these genes were performed to determine the molecular genetic defect in the patient.

Methods: Blood DNA was extracted and KCNJ11 \& INS coding regions were PCR amplified, purified and directly sequenced.

Results: A heterozygous INS p.L13R mutation was found, located within the signal peptide of preproinsulin. This variant is not found in dbSNP. No sequence change in $K C N J 11$ was detected.

Conclusions: The p.L13R missense mutation is a non-conservative change, affecting the highly conserved Leucine residue of preproinsulin signal peptide and this likely caused abnormal insulin trafficking/processing and led to the PNDM condition in the patient.

\section{EPO-062 Diabetes and Insulin}

HLA DQB1 and HLA DQA1 genotypes prevalence in children and adolescens with new-pnset diabetes type 1 in the Lower Silesia region

Teresa Zak' ; Malgorzata Malodobra²; Agnieszka Zubkieicz-Kucharska'; Anna Noczynska'; Tadeusz Dobosz ${ }^{2}$

${ }^{1}$ Medical University, Endocrinology and Diabetology for Children and Adolescents, Wroclaw, Poland; ${ }^{2}$ Medical University, 1. Molecular

Techniques Unit, Department of Forensic Medicine, Wroclaw, Poland

Background: The first degree relatives of patients with diabetes type 1 have increased genetic risk of developing clinical disease. In the population of Polish diabetic subjects the most frequent alleles are HLA- DRB $1 * 04, \mathrm{DRB} 1 * 03$, DQA1 and DQB1. The high-risk genotype may differ among populations.

Aim: The aim of this paper was to analyze prevalence of genetic risk factors in patients with new-onset diabetes in Lower Silesia and to compare the data with Polish population and Caucasian one.

Method: HLA DQB1 and HLA DQA1 genes were analyzed: SNP polymorphism of HLA DR gene locus was assessed using ABI 3130 gene analyzer with GENEscan software. The study involved 58 patients with new-onset diabetes type 1, 34 boys and 24 girls, 11 months to 18 years of age, admitted to the Dept. of Endocrinology and Diabetology for Children and Adolescence, Wroclaw Medical University, from June 2007 to May 2009.

\section{EPO-063 Diabetes and Insulin \\ Glycosylated haemoglobin as an indicator of metabolic regulation in children with diabetes mellitus type 1 on intensive treatment regimens with human insulin and with insulin analogues Diana Stimjanin-Koldzo'; Sabina Delic-Bikic ${ }^{1}$; Mirza Oruc ${ }^{2}$ \\ ${ }^{1}$ Cantonal hospital Zenica, Department of pediatrics, Zenica, Bosnia and Herzegovina; ${ }^{2}$ Faculty of health, Faculty of health, Zenica, Bosnia and Herzegovina}

Objective: This research determines the middle level of the HbA1c and analyses quality and quantity of applied insulin therapy, degree of puberty development, self-control and evaluates correlation between the level of metabolic regulation with every element of the therapy applied.

Methods: Patients are classificated into groups based on the average value of $\mathrm{HbAlc}$ and correlation of metabolic regulation with therapeutic principles. 67 patients have participated in the research, who were controlled during 2010 and 2011. average age $11,00 \pm 0,68$ years.

Results: The average duration of diabetes type 1 was $3,9 \pm 0,4$ years. The largest number of the examinees $(70,14 \%)$ had diabetes for 5 years, the smallest group $(10,44 \%)$ consisted of the examinees who had diabetes over 10 years. The average $\mathrm{HbA} 1 \mathrm{c}$ of the examinees was in range of excellent metabolic control of diabetes $(6,7 \pm 0.44 \%)$.According to the average HbAlc the patients in the research were divided in three groups with $1 \%$ range, and in two groups according to type of insulin in regime of intensified insulin therapy. Excellent metabolic control have achieved 23,8 \% (HbA1c $\leq 7 \%), 53,7 \%$ have achieved satisfying metabolic control (HbA1c 7,1-9\%), and 22,3\% had bad metabolic control (HbAlc $>9 \%)$. The gender didn't have effect unlike the age and puberty development, which was significant on metabolic regulation. The examines who had diabetes less then 5 years have achieved significantly better metabolic regulation. All the examinees were on intensified insulin therapy regime with average number of application of insulin which was $4 \pm 1.65,67 \%$ of the patients have replaced intensified human insulin therapy (average $\mathrm{HbA} 1 \mathrm{c} 8,6 \%$ ) with intensified therapy of insulin analogues (average HbAlc 8,4\%).

Conclusions: With this research we proved that better metabolic regulation of illness is on regime of the intensified insulin analogues therapy. Everyday adjustment of insulin doses is crucial condition for good metabolic regulation. 


\section{EPO-064 Diabetes and Insulin}

Does the increasement of diversity in blood glucose meters cause reliability problems?

Sevgi Akbel; Ebru Ercanli; Gul Yesiltepe Mutlu; Elif Ozsu; Filiz Mine Cizmecioglu; Sukru Hatun

Kocaeli University, School of Medicine, Pediatric Endocrinology and

Diabetes, Kocaeli, Turkey

Background: Within the last years in our country, new blood glucose meters have been begun to be used because of their low prices. However, the reliability of these devices has been debated. In this study, the compliance between the measurements with commonly used blood glucose meters and the venous blood sugar was evaluated.

Methods: Eighty five patients with type 1 diabetes whose mean age 13.0 \pm 3.4 years, mean $\mathrm{HbA1C} 8.2 \pm 1.9 \%$, mean duration of diabetes $4.4 \pm 3.6$ years were analyzed. Two diabetes nurses measured blood glucose with 5 different glucose meters (Accucheck, Glucoleader, Countor TS, IME-DC, Optium XCHEED) in addition to patients' glucose meters, simultaneously. At the same time their venous glucose levels were measured, also. The correlation between venous glucose levels and 6 different glucose meters was analyzed. Total number of errors were calculated and evaluated according to the American Diabetes Association's proposal. In the next stage cases were categorized according to their blood glucose levels $(<100,100-199,200-399,>400 \mathrm{mg} / \mathrm{dl})$ and evaluated according to "Consensus Error Grid" model.

Results: There was a very strong correlation between venous glucose levels and 4 glucose meters ( $\mathrm{r}$ values of patients' glucose meters, Accucheck, Glucoleader, Countor TS, IME-DC were $0.96,0.98,0.97,0.96,0.90$, respectively.. The mean glucose level measured by glucose meters was significantly lower than venous glucose level; the difference was 15.4, 11.4, 18.4, 34.7 and 16.3 for their self glucose meters, 11.4, 18.4, 34.7 and IME-DC, respectively. The $95 \%$ of glucose meter measurements were in the zone A+B, although $20 \%$ error repetition rate was below the $95 \%$ threshold value.

Conclusions: The correlation between venous and capillary glucose measurements was very strong/strong. The measurements of all glucose meters under real conditions were sufficent accurate according to "Consensus Error Grid" analyzes.

\section{EPO-065 Diabetes and Insulin}

\section{Severe hypoglycaemia in children with type 1 diabetes}

Jovana Dimic'; Silvija Sajic ${ }^{2}$; Ana Stankovic²; Vera Zdravkovic ${ }^{2}$

'University of Belgrade, Medical School, Belgrade, Serbia; ${ }^{2}$ University

Children Hospital, Endocrinology, Belgrade, Serbia

Background: Hypoglycemia is the most common acute complication of type 1 diabetes. Severe hypoglycemia $(\mathrm{SH})$ is a condition with altered mental status, coma and/or convulsions and the child is not capable to help himsef. As a consequence, some families have fear of hypoglycemia (FOH) which has the influence on the child's metabolic control.

Objective and hypotheses: To establish the frequency and highlight the importance of severe hypoglycemia in children with type 1 diabetes mellitus.

Methods: We conducted a survey of randomly selected patients in our clinic. We asked them about their diabetes duration, episodes of severe hypoglycemia, current insulin dose and $\mathrm{HbAlc}$ and collected data on their auxology. We divided patients into 2 groups: Group 1 (patients who did not experience severe hypoglycemia) and Group 2 (patients who had severe hypoglycemia). Results: We analyzed data for 31 patients, which represents about $10 \%$ of our clinic population. M/F ratio was $14 / 17$ and mean age of patients was $14,1 \pm 3,6$ years. The average duration of diabetes was $5,4 \pm 3,6$ years, $68 \%$ were in puberty and the average $\mathrm{HbAl} \mathrm{c}$ was $7,9 \pm 1,4 \% .39 \%$ of patients had at least one episode of $\mathrm{SH}$.

\begin{tabular}{lccc} 
Characteristics & Group 1 (19) & Group 2 (12) & $\mathbf{p}$ \\
\hline Age $(\mathrm{y})$ & $12,1 \pm 3,3$ & $16,1 \pm 3,3$ & $<0.01$ \\
\hline Gender (M/F) & $12 / 7$ & $3 / 9$ & $<0.05$ \\
Diabetes duration (y) & $4,1 \pm 2,8$ & $7,5 \pm 3,8$ & $<0.01$ \\
HbA1c (\%) & $7,6 \pm 1,5$ & $8,3 \pm 1,3$. & 0.23 \\
\hline Insulin dose (U/kg/day) & $1,1 \pm 0,3$ & $1,4 \pm 0,7$. & ns
\end{tabular}

The most frequent cause of SH was the exercise (50\%). SH occurred mainly during the night ( $01 \mathrm{~h}-05 \mathrm{~h})$ in $61,5 \%$ of patients. Majority of patients did not get glugagon injection $(75 \%)$.
Conclusions: We conclude that the risk of SH increases with the age and duration of diabetes. Those patients who experienced SH tend to have higher $\mathrm{HbA1c}$ which put them at risk of chronic complications.

\section{EPO-066 Diabetes and Insulin \\ Peculiarity requirements in the care of children less than 5 years with diabetes type 1}

Ahmed Salih Bendeddouche; Naouel Kaouadji; Feth-Allah Bassaid; Farah Ghellai; Yacoub Mansouri; Khadidja Bouriche; Sabrina Chefai Medical University of Tlemcen, Pediatrics, Algeria, Algeria

Introduction: The diabetes of the child is in the majority of the cases, a diabetes of type 1 insulinodependant of auto-immune origin. Its treatment aims at obtaining a glycemic control allowing to warn the arisen of the long-term complications.

Materials and methods: Cross-functional study of children of less than 5 years affected by diabetes type I, followed in the department of General Paediatrics EHS Tlemcen from 1/8/10 till 31/10/11. The study of the general characteristics are: age, sex, geographical origin, socioeconomic conditions, the pathological histories, the circumstances of discovery, the data of the clinical examination and the biological profits; The successful plans of insulin, the follow-up of the glycemic balance, the hygiéno-dietary rules and the regular control has the research for the hypoglycemic accidents and for the complications.

Results: 34 brought together observations.the department received these last 5 years 182 diabetics children among whom 59 diabetics of less than 5 years, over the period of 5 years and 13,77 for 100000 inhabitants in the year 2011, Sex ratio has 0,78 , average age of discovery in 25 months, family history is the circumstances of discoveries of diabetes in $63 \% ; 58 \%$ acidocétose, 29 $\%$ syndrome polyuro-polydyspsique and $4 \%$ by a cétose, $6 \%$ have a delay weight, 18 patients followed the conventional plan with mixture of insulin in 2 injections per day with an average glycemic balance to $6,24 \%$ of $\mathrm{HbA1C}$, 14 patients followed the plan in 4 injections with a glycemic balance of 3,12 $\%$, the same for 2 patients with 3 injections. The hypoglycemic accidents are noted in $59 \%$ of the cases

Conclusion: The diabetes type 1 of the child of less than 5 years became more frequent its coverage is more complex because of peculiarities bound to this age: a fast diminution of the residual insulinosecretion mostly without period of partial forgiveness, a sensibility brought up to the insulin Where from the necessity of a multidisciplinary coverage.

\section{EPO-067 Diabetes and Insulin \\ Report of a second Georgian patient with Wolfram Syndrome}

Irakli Paghava'; Alexi Mindadze²; Ketevan Gogolashvili ${ }^{3}$

"New Pediatric Clinic", Ltd., Pediatric Endocrinology, Tbilisi, Georgia

2"New Pediatric Clinic", Ltd., Pediatric Neurology, Tbilisi, Georgia;

3"New Pediatric Clinic", Ltd., Pediatric Ophtalmology, Tbilisi, Georgia

Background: Wolfram syndrome (WFS) is an autosomal recessive neurodegenerative disorder caracterized primarily by diabetes mellitus (DM), optic atrophy (OA), diabetes insipidus (DI), deafness (D).

Objective and hypotheses: This study reports Georgian WFS patient with remarkable ocular findings.

Methods: The estimated prevalence of WFS varied from 1/100,000 (estimate for the North America) down to 1/770,000 (UK). Georgia's population is about 4,500,000 and only one Georgian case of Wolfram syndrome was published before.

Results: 6.2 years old male was hospitalized with a preliminary diagnosis of DM Type 1 (based on the history of polyuria, polydipsia and weight loss; hyperglycemia and ketoacidosis). Visual acuity was reportedly reduced since infancy and was limited to counting fingers by the time of examination. History of congenital cataract and phacoaspiration OD was recorded. Ophthalmic examination revealed $\mathrm{OA}$ with visual acuity $0.02 \mathrm{OU}$, postoperational aphacia OD with normal lens OS, high-degree myopia OS. A diagnosis of the WFS was established based on the constellation of the juvenile-onset insulindependent DM and OA. Neither DI (no polyuria when euglycemic, urine SG 1015) nor renal tract abnormalities were detected. Severe rotatory nystagmus was the only focal neurological impairment established. Mild cognitive impairment and behavioral deviations were noted (possibly secondary to the lack of specialized training for visual impairment). Insulin requirement within 
2 weeks after the DM onset was $0.18 \mathrm{U} / \mathrm{kg} / 24 \mathrm{hrs}$.

Conclusions: The early (congenital?) development of optic nerve atrophy preceeding DM by at least 6 years was noteworthy. Bilateral congenital cataract was established in 5\% of 59 WFS patients by Chaussenot A. et al. in 2011. However, our WFS patient presented the history of the unilateral cataract, the other lens being intact. We recommend ophthalmic examination at first admission for all patients with insulin-dependent DM.

\section{EPO-068 Diabetes and Insulin}

Quality of life and metabolic control in children and adolescents with type 1 diabetes mellitus Irina Osokina

State Research Institute for Medical Studies of the North,

Endocrinology, Krasnoyarsk, Russian Federation

Background: Quality of Life is considered being an important factor to measure since it often correlates with children's and adolescents' perception of having diabetes, diabetes-related conflicts within the family, depression andglycemic control.To evaluate quality of life (QOL) in children with type 1 diabetes (DM) in relation to metabolic control.

Methods: The cross-sectional study included 56 children with type $1 \mathrm{DM}$ (aged 5-18 years) and their parents. QOL was evaluated by the PedsQL 3.0 Diabetes Module. The children were assigned to one of two groups according to metabolic control. Metabolic control was assessed by a single $\mathrm{HbAlc}$ at the time of the visit.

Results: Children with lower HbAlc (less than 8.0\%) reported that their QOL on several domains was better than that of children with poor metabolic control. By self-report, children with better metabolic control reported that they had statistically significant better QOL scores for the diabetes symptoms, treatment barriers, treatment adherence and worry subscales in comparison to the children with worse metabolic control. Children with lower HbAlc differ significantly in the same diabetes related subscales from children who have poor metabolic control. The only exception is the communication subscale of the child and parents report where there is no difference between the groups. The parents experienced that their children had lower diabetes related Quality of Life compared to the children themselves, $\mathrm{P}<0.05$.

Conclusions: Children with better metabolic control report better QOL.

\section{EPO-069 Diabetes and Insulin}

\section{Comparative assessment of achievement of target levels of therapy in type 1 diabetes using various kinds of insulin}

Alimova Nasiba; Rakhimova Gulnara; Bakhadirov Said

RSSPMC of Endocrinology, Child Endocrinology, Tashkent, Uzbekistan

Study objective: Comparative assessment of achievement of target levels of therapy at type 1 diabetes using various kinds of insulin in out-patient conditions.

Materials and methods: Since 2007, within the the international grant «Life for a child» (LFC), monitoring of children and teenagers with type 1 diabetes is carried out. In 2007, 43 patients from Tashkent and Tashkent area were included into the project "LFC". Of them, 20 children and teenagers with type 1 diabetes receive Lantus and Actrapid within the last 3 years, and 20 children and teenagers receive Protofan and Actrapid.

Study results: The mean age of patients receiving Lantus + Actrapid was $15.2 \pm 1.0$ years, duration of the disease $7.1 \pm 1.0$ years. The mean age of the patients receiving Protofan + Actrapid was $17.1 \pm 0.9$ years, duration of the disease $-9.3 \pm 0.7$ years. Improvement of height-weight indices in both groups was noticed. The comparative analysis of use of various kinds of insulin showed, that in patients receiving insulin Lantus, are better compensated (average Hbalc level was $7.4 \pm 0.2 \%$ ) in comparison with children, receiving usual insulins (average HbAlc level $-8.0 \pm 0.2 \%$ ). HbAlc decreased by 1.5 on the background of the intensified insulin therapy with Protofan, and by 1.6 in patients receiving Lantus. Daily Lantus dose was by $6.7 \%$ less than dose of Protofan. The requirement for insulin in both cases increased by $0.1 \%$. Acute complications of type $1 \mathrm{DM}$ have decreased. Among children and teenagers receiving Protofan, frequency of hypoglycemias was high. Frequency of ketoacidotic conditions decreased in both cases by $5.6 \%$ and $10.7 \%$ accordingly. Conclusions: Thus, use of insulin Lantus showed the advantage against NPH insulin. There was improvement of carbohydrate metabolism indices against smaller number of hypoglycemias in children and teenagers with type $1 \mathrm{DM}$.

\section{EPO-070 Diabetes and Insulin}

Frequency of vaginal candida colonization and relationship between metabolic parameters in patients with type 1 diabetes mellitus Nesibe Akyürek; Beray Selver Ekliodlu; Mehmet Emre Atabek; Sevil Aru Yuca

Necmettin Erbakan University School of Medicine, Div of Pediatric

Endocrinology, Konya, Turkey

Background and objective: Patients with diabetes mellitus (DM) are at risk for vulvovaginal candidasis. The aim of this study was to determine the species-specific prevalence rate and risk of candidiasis in patients with type $1 \mathrm{DM}$.

Methods: Children aged between 8-16 years were included in the study. Clinical and laboratory features of diabetes and evidence of genital symptoms were recorded. Vaginal swabs were taken from patients and placed on Sabouraud's dextrose agar and incubated. Following a 12- hour nighttime fasting venous blood samples were taken simultaneously for analyses of blood glucose, $\mathrm{HbA} 1 \mathrm{c}$ and lipid profile. Simple one-time fluconazole treatment regimen used to treat patients with vulvo-vaginal candidiasis.

Results: Candida spesies were isolated in 30 of $76(\% 39,4)$ swabs of patients with type $1 \mathrm{DM}$. Subjects who had candida colonisation were all acute. The predominat candida species isolated from patients with type $1 \mathrm{DM}$ were C.albicans $50 \%$, C. glabrata 36,6 \%, C.crusei 3,3\%, C.spesies 6,6\% , C.dubliniensis $3,3 \%$. Subjects with vulvo-vaginal candidiasis had higher mean $\mathrm{HbA1} \mathrm{c}$ when compared to those who had no such infection ( $\mathrm{p}=0.047$ ). Conclusions: There seems to be a significant link between hyperglycaemia and vulvo-vaginal candidiasis in patients with type $1 \mathrm{DM}$. Improving glucose control may reduce the risk of Candida colonisation and potentially symptomatic infection, among children with diabetes.

\section{EPO-071 Diabetes and Insulin \\ What is the contribution of insulin analogues in the metabolic control of type 1 diabetes in children? \\ Mongia Hachicha; Sana Kmiha; Lamia Sfaihi; Hajer Aloulou; Thouraya Kamoun \\ CHU Hedi Chaker, Department of Pediatrics, Sfax, Tunisia}

Background: Diabetes mellites type 1 (T1DM) in children is characterized by unstable course. A significant number of studies shows that introduction to insulin analogues treatment aims towards better control of the disease.

Objective and hypotheses: The assessment of metabolic control in children with T1DM that were introduced to insulin analogue treatment after many years of treatment with classic (human) insulin.

Methods: The study included 24 patients 6-20 years old $(13.3+/-3.4)$ with T1DM, transferred from treatment with human insulin to insulin analogues treatment. Data were obtained directly from patients and their parents, as well as from medical records.

Results: In 9 examinees (37.5\%), before the introduction to insulin analogue treatment, severe hypoglicemia was registered, while after it was not registered $(\mathrm{p}<0.001)$. Ketoacidosis, before introduction to insulin analogues treatment, appeared in 9 examinees (37.5\%), and after the introduction to insulin analogue treatment it appeared in only one examinee (4.1\%). The introduction to insulin analogues treatment, leads to a decrease in the value of glycolized haemoglobin (HbA1c) after 1 year $(10.12+/-1.76 \%$ vs $9.05+/-1.69 \%$, $\mathrm{p}=0.06$ ). Average daily dose of insulin expressed per IU $/ \mathrm{kg}$ of classic insulin was $1.4+/-0.28$ versus $0,90+/-0.20(p>0.05)$ after insulin analogue.

Conclusions: The use of insulin analogue treatment in childhood provides adequate metabolic control and substantially reduces the risk of acute complications (severe hypoglicemia, ketoacidosis). 


\section{EPO-072 Diabetes and Insulin}

Effect of coeliac seropositivity in Russian children with diabetes mellitus type 1

Elena Hennessy'; Tamara Kuraeva'; ; Sergey Prokofev; Valentina Peterkova'

'Endocrinology Research Centre, Institute of Children Endocrinology, Moscow, Russian Federation; ${ }^{2}$ Endocrinology Research Centre, Laboratory of immunology, Moscow, Russian Federation

Background: Association of diabetes mellitus type 1 (DM1) and celiac disease is well known in the world. However, there are not so many studies about effect of celiac seropositivity in Russian children with diabetes mellitus type 1.

Objective and hypotheses: To estimate the prevalence of celiac antibodies in Russian children with DM1 and compare seropositive and seronegative groups of patients.

Methods: Antigliadin (AGA) and tissue transglutaminase antibodies (tTG) were investigate in 499 russian children with DM1. Group $1(\mathrm{n}=37)$ - seropositive patients $(\mathrm{AGA}>12 \mathrm{U} / \mathrm{ml}, \mathrm{tTG}>10 \mathrm{U} / \mathrm{ml})$. Group $2(\mathrm{n}=163)$ - group of seronegative patients comparable in age and duration of DM1 with seronegative groups.

Results: There were $7,4 \%$ seropositive patients $(\mathrm{AGA}>12 \mathrm{U} / \mathrm{ml}, \mathrm{tTG}>10 \mathrm{U} /$ $\mathrm{ml}$ ) in our study. $3,2 \%$ of patients had tTG $>10 \mathrm{U} / \mathrm{ml}$ regardless the presence of elevated AGA and $1,6 \%$ of patients had significant elevation of $\mathrm{tTG}>100$ $\mathrm{U} / \mathrm{ml}$. We found that patients with DM1 and celiac antibodies had gastrointestinal symptoms more often than seronegative patients $(29,7 \%$ vs $11,7 \%)$ $(p<0,05)$. Seropositivity does not affect growth, weight and metabolic control (HbA1c and insulin requirement). The blood levels of vitamin D were the same in all groups of patients. Downward trend in bone mineral density Zscore was found in patients with celiac antibodies $(p=0,07)$. Children with celiac antibodies had signs of iron deficiency anemia significantly more often than seronegative patients $(24,3 \%$ vs $8,6 \%)(p<0,05)$.

Conclusions: High-titer seropositivity to celiac antigens is associated with gastrointestinal symptoms, anemia and reductions in bone mineral density in children with DM1, justifying screening of high-risk patients.

\section{EPO-073 Diabetes and Insulin}

\section{Hyperglycaemia and beta-cell antibodies: is always pre-type 1 diabetes?}

Giuseppe d'Annunzio; Marta Marchi; Alessandro Salina; Concetta Aloi; Renata Lorini

IRCCS Gaslini, Pediatric Clinic, Genoa, Italy

Background: Type 1 Diabetes Mellitus (T1DM) results from autoimmune destruction of insulin-producing beta cells of the pancreas. Maturity-onset diabetes of the young (MODY) is a heterogeneous group of monogenic disorders with at least eleven different genes leading to alterations in insulin secretion. In particular, heterozygous mutations in the glucokinase gene (GCK) result in MODY subtype GCK or MODY2, one of the most common forms of MODY Southern Europe.

Hypothesis: The association between MODY and T1DM has been previously attributed to a misdiagnosis.

Population: A 10 years old Italian girl was admitted for incidental hyperglycemia and strong family history for diabetes mellitus and coeliac disease. Results: Fasting hyperglycemia $(6.2 \mathrm{mmol} / \mathrm{L}$, n.v. $<5.5)$, increased $\mathrm{HbA} 1 \mathrm{c}$ $6,6 \%$ (n.v. 4-6), a weak positivity for GADA $(1,77 \mathrm{U} / \mathrm{mL}$; n.v. $0-0,9)$ without IAA were observed. Oral glucose tolerance test showed impaired glucose tolerance, and IVGTT showed a low FPIR. Subsequent disappearance of GADA, stability of glycemic levels and family history for mild hyperglycemia induced us to consider a clinical diagnosis of MODY. Genetic analysis, performed by direct sequencing, showed a GCK missense mutation (c.626C > T p.T209M) in both proband and her mother. This change was previously found in 6 Caucasian families and in one from Chile.

Conclusions: Incidental hyperglycemia represents a diagnostic challenge. Not only pre-type 1 diabetes, but also monogenic forms are characterized by initial mild hyperglycemia. Therefore we suggest to reconsider genetic testing for MODY in case of suspected diagnosis of MODY with weak beta-cell autoimmunity.

\section{EPO-074 Diabetes and Insulin}

Lower frequency of insulin pump treatment in type 1 diabetic children and adolescents with Turkish background

Andrea Icks' ; Oliver Razum²; Joachim Rosenbauer'; Christina Bächle ${ }^{1}$; Andreas Hungele ${ }^{3}$; Kirsten Mönkemöller ${ }^{4}$; Esther Müller-Godeffroy ; Bettina Heidtmann ${ }^{6}$; Thomas Kapellen; Esther Moltz ; Reinhard W Holl

${ }^{1}$ German Diabetes Center, Institute of Biometrics and Epidemiology, Düsseldorf, Germany; ${ }^{2}$ Bielefeld University, Department of

Epidemiology \& International Public Health, School of Public Health, Bielefeld, Germany; ${ }^{3}$ University of Ulm, Institute of Epidemiology and Medical Biometry, Faculty of Medicine, Ulm, Germany; ${ }^{4}$ Hospitals of the City of Cologne, Children's Hospital, Cologne, Germany; ${ }^{5}$ University Hospital of Schleswig-Holstein, Department of Pediatrics and Adolescent Medicine, Lübeck, Germany; ${ }^{6}$, Catholic Children's Hospital Wilhelmstift, Hamburg, Germany; ${ }^{7}$ University Hospital of Leipzig, University Clinic and Policlinic for Children and Adolescents, Leipzig, Germany

Background: Little is known about diabetes care processes in pediatric diabetes patients with migration background.

Objective and hypotheses: Aim of our study was to investigate whether the proportion of insulin pump therapy in pediatric patients with type 1 diabetes differs between children and adolescents with Turkish origin and those without migration background in Germany.

Methods: In 2009 data of a nationwide pediatric diabetes documentation program (DPV), we identified patients with Turkish background using a name algorithm and patients' and parents' country of birth, and excluded patients with origin from other countries. We estimated the prevalence of insulin pump therapy in patients aged $<20$ years with Turkish origin and those without migration background using logistic regression, adjusting for potential confounders for age, sex, diabetes duration, BMI, and HbAlc.

Results: We included 997 pediatric type 1 diabetes patients with Turkish background and 15664 patients without migration background (age 12.2 \pm 4.1 and $12.4 \pm 4.2$ years; $49.5 \%$ and $52.9 \%$ male, BMI-SDS $0.62 \pm 0.96$ and $0.44 \pm 0.91$, $\mathrm{HbAlc} 8.1 \pm 1.6 \%$ and $7.9 \pm 1.5 \%$ ). The adjusted prevalence of insulin pump therapy among patients with Turkish origin $( \pm \mathrm{SE}$ ) was $18.0 \pm 0.02 \%$, among patients without migration background $30.2 \pm 0.01 \%(\mathrm{p}<0.001)$.

Conclusions: The prevalence of insulin pump therapy in pediatric patients with type 1 diabetes is roughly half among patients with Turkish background compared to those without migration background. This could not be explained by differences in age, diabetes duration, or $\mathrm{HbAlc}$. Lower acceptance of new therapies, language barriers, different disease belief or, on the other hand, factors within the health care system may play a role. This has to be examined in further research. The study was supported by the Competence Network Diabetes mellitus funded by the Federal Ministry of Education and Research (FKZ 01GI0802, 01GI0859).

\section{EP0-075 Diabetes and Insulin \\ ABCC8 gene mutations in patients with neonatal diabetes and congenital hyperinsulinism}

Magdalena Avbelj'; Katarina Trebusak Podkrajsek ${ }^{2}$; Natasa Bratina'; Tinka Hovnik'; Mirjana Perkovic Benedik ${ }^{3}$; Mojca Zerjav Tansek'; Nina Bratanic ${ }^{\prime}$; Tadej Battelino ${ }^{\prime}$

'University Children's Hospital Ljubljana, Department of Pediatric Endocrinology, Diabetes and Metabolic Diseases, Ljubljana, Slovenia; 2University Children's Hospital Ljubljana, Unit for Special Laboratory Diagnostics, Ljubljana, Slovenia; ${ }^{3}$ University Children's Hospital Ljubljana, Department of Developmental, Child and Adolescent Neurology, Ljubljana, Slovenia

Background: Sulfonylurea receptor 1 (SUR1) encoded by ABCC8 gene, and inward rectifier KIR6.2 encoded by KCNJ11 gene, constitute ATP-sensitive $\mathrm{K}+$ channel in pancreatic $\beta$-cells responsible for regulation of glucose-induced insulin secretion. Loss of function and activating mutations in respective genes are associated with hyperinsulinism of infancy (HI) and neonatal diabetes (NDM) with/without associated neurological disorder, respectively. Objective and hypotheses: The possible implication of ABCC 8 and KCNJ11 gene mutations in Slovenian patients with the two rare congenital disorders in insulin regulation was evaluated. 
Methods: Five patients with NDM and 3 patients with HI were screened for mutations in $\mathrm{ABCC} 8$ and $\mathrm{KCNJ} 11$ genes by direct sequencing of the whole coding region and exon-intron boundaries.

Results: A novel homozygous ABCC8 gene mutation p.Gly316Trp was identified in one patient with permanent NDM requiring insulin therapy since the age 2,5 months and otherwise normal development. At the age of 19 years he had well controlled diabetes using only glimepiride. In one of the HI patients a heterozygous $\mathrm{ABCC} 8 \mathrm{p}$.Ser1386Phe mutation was identified, inherited from the father diagnosed with type 2 diabetes. The patient had hyperinsulinemic hypoglycemia since neonatal period, developed transient diabetes with insulinopenia at the age of 25 years and suffers from epilepsy, mild to moderate mental retardation and psychiatric disorder marked with aggressiveness and agitation. No other mutations in either $\mathrm{ABCC} 8$ or KCNJ11 were detected in this small cohort of patients.

Conclusions: The mutations in $\mathrm{ABCC} 8$ and $\mathrm{KCNJ} 11$ do not appear to be a frequent cause of congenital disorders in insulin regulation in Slovenian patients. A novel mutation in ABCC8 gene in NDM was identified. While p.Ser1386Phe ABCC8 mutant has been reported previously as an autosomal dominant cause of HI, incomplete penetrance of the disease in our case suggests the heterozygous mutation alone is not sufficient to result in a severe phenotype.

\section{EPO-076 Diabetes and Insulin \\ Insulin oedema in newly diagnosed type 1 diabetes mellitus \\ Ayse Derya Bulus; Nesibe Andiran \\ Keçiören Training and Education Hospital, Pediatric Endocrinology, Ankara, Turkey}

Background: Insulin is essential the treatment type 1 diabetes. Rarely, significant fluid retention can occur with insulin therapy, resulting in an oedematous state which can vary from mild peripheral oedema to cardiac failure and massive serosal effusions. Here we report a 12-years-old boy with newly diagnosed type 1 diabetes who presented with oedema of the lower extremities approximately one week after the initiation of insulin treatment.

Case: A 12-year-old boy was admitted with an acute presentation of DKA. On physical examination height was $152 \mathrm{~cm}(-0.31 \mathrm{SDS})$ and his weight 30 $\mathrm{kg}(-1.75 \mathrm{SDS})$, he had somnolance he had mild dehydration. Laboratory findings glucose $800 \mathrm{mg} / \mathrm{dL}$, ketonuria with acidosis ( $\mathrm{pH}$ of 7.0) and $\mathrm{HbAlc}$ concentration of $16 \%$. After DKA treatment firstly regular insülin started and on 4 days later taking insulin lispro 7-8 $\mathrm{U}$ before meals and glargine $14 \mathrm{U}$ before bed time. On the 3rd day of this regimen; oedema initially involved his ankles but progressed to her legs, thighs and developed her abdomen and face His gaimed $10 \mathrm{~kg}$ in the following 5 days. Serum albumin levels decreased from 3.5 to $3.2 \mathrm{~g} / \mathrm{dL}$, with mild proteinuria. Transaminases elevated mildly. Renal and thyroid function were normal. Chest X-ray, ecocardiography and abdominal ultrasound findings were normal. Seven days later the oedema had completely resolved with fluid and salt restriction.

Discussion: Insulin oedema is probably an underreported physical finding following initiation of insulin therapy. Insulin oedema in type $1 \mathrm{DM}$ was reported rarely. These patients typically presented between 2 and 4 weeks following an initial presentation of DKA. Diuretic therapy may be indicated in more severe decompensated cases.

\section{EPO-077 Diabetes and Insulin \\ Two patients with Wolfram syndrome showed different presentations}

Zeynep Siklar'; Gönül Ocal'; Merih Berberoglu';

Bülent Hacihamdioglu'; Senay Savas Erdeve'; Emine Camtosun';

Pinar Kocaay'; Sarah E Flanagan; Sian Ellard ${ }^{2}$

${ }^{1}$ Ankara University School of Medicine, Pediatric Endocrinology,

Ankara, Turkey; ${ }^{2}$ Peninsula Medical School, Barrack Road, University

of Exeter, Institute of Biomedical and Clinical Science,, Exeter, United Kingdom

Background: Wolfram syndrome (WS) is a neurodegenerative disorder characterized by different clinical manifestations, such as diabetes mellitus, optic atrophy, diabetes insipidus, deafness, neurological symptoms, urinary dysfunction, gastrointestinal dysfunction and psychiatric manifestations.

Objective and hypotheses: Clinical presentations and begining time of the symptoms can be various.
Methods: Two patients with WS were presented.

Results: Case 1: 1.7 years of age male patient admitted to our clinic with complaints of hyperglycemia and ketoacidosis. He had been diagnosed as congenital hydrocephalia, agenesis of corpus callosum, duodenal atresia, and congenital primary hypothyroidism. He had autoimmune negative diabetes mellitus. Two cousins of patient had been diagnosed clinically as WS. In this case, a homozygous frameshift mutation, V412fs, at exon 8 in WFS1 gene was detected. We have been unable to find any reports of patients with duodenal atresia or hydrocephalus in association with Wolfram syndrome. Case 2: 3.3 years of age female patient admitted to clinic with hyperglycemia. Diabetes autoantibodies were negative on admission. There is not any other pathological findings. At 7 years of age diabetes antibodies found as positive, and patient had been thought as late onset autoimmune diabetes (LADA). But at 14 years of age, optic atrophy, sensorineural deafness, and central diabetes insipidus were added to clinical findings. This case emphasize that the component of WS can appear with time

Conclusions: The differential diagnosis of autoimmune negative diabetes including WS can not be possible on admission for each case. Molecular analysis is very helpfull for this. In Case 1, it is possible that these extra features may be due to a mutation in a second unrelated gene such as GLIS or that they can be rare features associated with WS. The components of WS can appear in course of time as in Case 2. Monitoring of diabetic patient in terms of additional symptoms, in spite of autoantibody positivity, can be recommended.

\section{EP0-078 Diabetes and Insulin \\ Disordered eating in Greek adolescents with type 1 diabetes mellitus (T1DM): a preliminary report \\ Betina Kandyla' ; Artemis Tsitsika²; Elena Critselis²; Chara Tzavara²; Chryssa Bacoula ${ }^{3}$; Kyriaki Karavanaki' \\ 'University of Athens, Diabetic Clinic, "P\&A Kyriakou" Children's Hospital, Second Pediatric Department, Athens, Greece; ${ }^{2}$ University of Athens, Adolescent Health Unit, "P\&A Kyriakou" Children's Hospital, Second Pediatric Department, Athens, Greece; ${ }^{3}$ University of Athens, "P\&A Kyriakou" Children's Hospital, Second Pediatric Department, Athens, Greece}

Background: Previous studies report a higher prevalence of disturbed eating behaviours among adolescents with T1DM, such as bulimia nervosa (BN), binge eating disorder and food restriction diet, compared to healthy peers, while anorexia nervosa is rare. There are no relative data up todate concerning T1DM adolescents in Greece.

Objective and hypotheses: Study of eating disorders and body image in T1DM adolescents versus a healthy control group.

Methods: We studied 69 adolescents aged $($ mean \pm SD) $16.3 \pm 2.0$ years. Among them, $23 \mathrm{~T} 1 \mathrm{DM}$ adolescents (age: $16.8 \pm 2.7$ years, disease duration:6.29 \pm 2.9 years, $\mathrm{HbAlc}: 9.22 \pm 1.58 \%$ ) were compared to 46 healthy controls, matched for age, gender and socio-economic level. Anonymous self-completed questionnaires for the evaluation of body image and the Eating Attitudes test (EAT26) were used to estimate eating disorders.

Results: T1DM adolescents were more satisfied with their body $(95.7 \%$ vs $71.1 \%, \mathrm{p}=0.025)$ and fewer wanted to change their somatotype $(34.8 \%$ vs $63 \%, p=0.026$ ). When compared to controls, T1DM adolescents found themselves preoccupied with food more often $(\mathrm{p}=0.050)$ and were more aware of the caloric content of foods that they consumed $(p=0.013)$. They also frequently avoided food containing sugar $(\mathrm{p}<0.001)$ and consumed dietary food $(\mathrm{p}=0.001)$. On the contrary, a significantly smaller percentage of adolescents with diabetes preferred to have "empty stomach feeling" $(\mathrm{p}=0.002)$ and had the impulse to vomit after meals $(\mathrm{p}=0.044)$. However, using a logistic regression analysis with T1DM being the dependent variable, no significant difference in the likelihood of eating disorders between the two study groups was found (food restriction: $\mathrm{p}=0.350$, bulimia and food preoccupation: $\mathrm{p}=0.890$, or food intake control: $\mathrm{p}=0.228$ ).

Conclusions: T1DM adolescents were found to be more satisfied with their body image, and more preoccupied with the quality of food and the amount of included calories. They also had less inappropriate weight losing compensatory behaviour. 


\section{EPO-079 Diabetes and Insulin}

Insulin pumps - panacea for children with type 1 diabetes mellitus (T1DM)?

Anna Malczewska'; Barbara Nowak'; Ewa Barg ${ }^{2}$

'Wroclaw Medical University, Students Science Association of

Endocrinology and Diabetology of Adolescence, Wroclaw, Poland

${ }^{2}$ Wroclaw Medical University, Endocrinology and Diabetology for

children and adolescents, Wroclaw, Poland

Background: In recent years popularity of insulin pumps (CSII) as method of intensive insulin therapy in children with T1DM is constantly rising.

Objective and hypotheses: The comparison of influence of CSII and Multiple Daily Injections (MDI) in children with T1DM on their metabolic control, process of their growth and evaluation of seasonable variability of $\mathrm{HbA} 1 \mathrm{c}$ in these children.

Methods: 71 children with T1DM (29 boys) (4-19 years) (mean age: 13,823,95 years). Metabolic control was assessed on basis of HbAlc serum concentration and process of growth on basis of height and body weight measures. Children were divided into 4 groups due to time from T1DM diagnosis to CSII initiation: up to 1 year, 1-3 years, 3-6 years and over 6 years. To evaluate seasonable variability of $\mathrm{HbA} 1 \mathrm{c}$ in period 1.01.2006-12.12.2010 $\mathrm{HbA} 1 \mathrm{c}$ from 69 children were used.

Results: Mean duration of MDI:3,05 $\pm 3,14$ years, CSII:2,79 $\pm 1,89$ years Mean $\mathrm{HbA1c}$ while MDI was 7,62 $\pm 1,44$, in 1st year of CSII:7,44 $\pm 1,46$ $(-2,36 \%$ compared with MDI); in 2nd year of CSII:7,57 $\pm 1,23(-0,66 \%)$ and in 3rd year of CSII:7,66 $\pm 1,3(+0,52 \%)$. During MDI and CSII mean HbAlc was rising. Children who started CSII up to 1 year had the significantly lowest CSII mean HbA1c (7,42 $\pm 1,04)$. Mean height SDS in 1st group:0,85 $\pm 1,63$, mean BMI SDS:1,91 $\pm 2,32$-in other groups these values were crucially lower. At T1DM diagnosis mean height SDS:0,38 $\pm 1,27$. Along MDI height SDS was decreasing and after CSII initiation-rising. Mean CSII height SDS was importantly higher than while MDI $(0,35 \pm 1,33$ vs. $0,20 \pm 1,16)$, like mean BMI SDS $(1,21 \pm 1,87$ vs. $0,49 \pm 1,25)$. HbAlc and height SDS correlated significantly $(r=-0,16, p=0)$. The lowest level of HbAlc was in July $(7,130,95)$, and the highest in November $(7,71,76)$.

Conclusions: 1 . Children improved their metabolic control (MC) in the first 2 years of CSII. 2. Time passing worsened MC during CSII and MDI. 3. Due to CSII children gained greater increases in height and body weight. 4. Summer holidays positively influenced for MC.

\section{EPO-080 Diabetes and Insulin}

\section{Friedreich ataxia and diabetes mellitus}

Angeliki Pappas; Annette Simon; Anja Spruenken; Stefan Wueller; Norbert Wagner

University hospital RWTH Aachen, Department of pediatrics, Aachen, Germany

Background: Friedreich ataxia (FRDA) is the most common hereditary ataxia and is characterized by progressive degeneration of the central and peripheral nervous systems, cardiomyopathy and increased incidence of diabetes mellitus. $10 \%$ have diabetes and $20 \%$ have another glucose homeostasis disorder. Both insulin resistance and beta-cell dysfunction are implicated, without serologic evidence for autoimmune destruction.

Results: We report about our 17 years old patient with diagnosed FRDA who was found to have hyperglycemia in a routine check-up. Retrospectively he reported polyuria and polydipsia. At the time of hospitalization there was evidence of ketonuria. The C-peptid level was in low-normal range. No islet-, GAD, IA2-antibodies were found. BMI was elevated with $27 \mathrm{~kg} / \mathrm{m} 2$. Insulin therapy was started, $\mathrm{Hb}$ Alc went down from $10,4 \%$ to $5,7 \%$ after 3 months. Weight went up to BMI $30 \mathrm{~kg} / \mathrm{m} 2$ and metformin was started. We will report about the further metabolic development.

Conclusions: Diabetes mellitus in Friedrich ataxia is based on loss of betacell function, but symptoms at manifestation can be mild. Oral antidiabetics can help to control the metabolic disorder. Check for glucosuria every 6 months can be recommended in asymptomatic patients as well as prevention of obesity.

\section{EP0-081 Diabetes and Insulin}

The timing of thyroid function tests in patients with newly diagnosed type 1 diabetes mellitus Selim Kurtoglu; Leyla Akin; Mustafa Kendirci; Nihal Hatipoglu Erciyes University Faculty of Medicine, Pediatric Endocrinology, Kayseri, Turkey

Background: Autoimmune thyroid disorders can be associated with type 1 diabetes mellitus. Therefore, it is advised that thyroid function tests should be assessed at the diagnosis and annually thereafter. However there is not a certain time recommended to make the measurements.

Objective and hypotheses: To investigate the effect of the timing of blood sampling on thyroid function tests in patients with newly diagnosed type 1 diabetes mellitus (NDDM).

Methods: A total of 68 patients with NDDM (43 males, 24 females, median age; 10.2 years (1.1-17.1)) were included in the study. In all patient thyroid function tests were measured at a time during their admission. Abnormal results were revaluated after 3 weeks to 3 months.

Results: Totally, 33 of 68 patients had abnormal thyroid functions at the onset of diagnosis. None of these patients required treatment. Only one of them had still abnormal results at 3 months. The frequency of abnormal thyroid functions were significantly higher in patients who were tested in the first three days than those tested thereafter $(27 / 35$ vs $1 / 9 ; p=0.02)$.

Conclusions: Transient abnormalities of thyroid function are common at the onset of type 1 diabetes mellitus. Thyroid hormones should be better to be measured at least three days after the diagnosis.

\section{EPO-082 Diabetes and Insulin \\ Comparative assessment of achievement of target levels of therapy at type 1 diabetes using various kinds of insulin}

Rakhimova Gulnara; Alimova Nasiba; Bahadirov Said

RSSPMC of Endocrinology, Paediatric Endocrinology, Tashkent, Uzbekistan

Study objective: Comparative assessment of achievement of target levels of therapy at type 1 diabetes using various kinds of insulin in out-patient conditions.

Materials and methods: Since 2007, within the the international grant «Life for a child» (LFC), monitoring of children and teenagers with type 1 diabetes is carried out. In 2007, 43 patients from Tashkent and Tashkent area were included into the project "LFC". Of them, 20 children and teenagers with type 1 diabetes receive Lantus and Actrapid within the last 3 years, and 20 children and teenagers receive Protofan and Actrapid.

Study results: The mean age of patients receiving Lantus + Actrapid was $15.2 \pm 1.0$ years, duration of the disease $7.1 \pm 1.0$ years. The mean age of the patients receiving Protofan + Actrapid was $17.1 \pm 0.9$ years, duration of the disease $-9.3 \pm 0.7$ years. Improvement of height-weight indices in both groups was noticed. The comparative analysis of use of various kinds of insulin showed, that in patients receiving insulin Lantus, are better compensated (average $\mathrm{HbAlc}$ level was $7.4 \pm 0.2 \%$ ) in comparison with children, receiving usual insulins (average HbA1c level $-8.0 \pm 0.2 \%$ ). HbA1c decreased by 1.5 on the background of the intensified insulin therapy with Protofan, and by 1.6 in patients receiving Lantus. Daily Lantus dose was by $6.7 \%$ less than dose of Protofan. The requirement for insulin in both cases increased by $0.1 \%$. Acute complications of type $1 \mathrm{DM}$ have decreased. Among children and teenagers receiving Protofan, frequency of hypoglycemias was high. Frequency of ketoacidotic conditions decreased in both cases by $5.6 \%$ and $10.7 \%$ accordingly. Conclusions: Thus, use of insulin Lantus showed the advantage against NPH insulin. There was improvement of carbohydrate metabolism indices against smaller number of hypoglycemias in children and teenagers with type $1 \mathrm{DM}$.> 
EPO-083 Diabetes and Insulin

Diabetic ketoacidosis in children: retrospective study

Zineb Imane; Hanane Khabba; Samah Amhager; Naima Bennani; Ahmed Gaouzi; Amina Balafrej

Children Hospital of Rabat, Unit of Pediatric Endocrinology /

Diabetology, Rabat, Morocco

Background: Diabetic ketoacidosis is a metabolic emergency and a major cause of morbidity and mortality in children with diabetes.

Objective: The aim of our study is to evaluate the epidemiological, clinical, biological, evolutionary, and the therapeutic management of children with DAC hospital.

Methods: Our retrospective study concerns the study of 79cas acid-ketotic decompensation admitted to the pediatric diabetes unit at Children's Hospital of Rabat during the years 2008-2009. Our study is based on the analysis of anamnestic, clinical, laboratory and treatment of each case.

Results: The age of our patients ranged between 7 months and 16years 4 months with two peaks of high incidence of ketoacidosis: the first in children under 5 and the second between ages 11-16years, no sex predominance. The study shows a family of first-degree consanguinity in $21 \%$ of cases, diabetes in $40 \%$ of familial cases. Ketoacidosis is opening in $83.5 \%$ of cases. The clinical symptoms are varied: polyuria-polydipsia syndrome was found in $89 \%$ of patients followed by digestive disorders. Disorders of consciousness are present in $28 \%$ of our patients with three cases of coma. The hydration status was correct in $62 \%$ of cases. -Triggers are dominated by infectious causes and treatment discontinuation. Treatment consisted of adequate rehydration combined with insulin therapy according to the protocol. The average time to resolve ketoacidosis was de23.25heures. -The hospital stay averages 12 days, with extremes ranging from 3 to 24 days. The outcome was favorable in all cases outside of a death.

Commentary: Our study aims to highlight that the diagnosis of DKAshould be started early and requires a well codified treatment. Hence the importance of awareness and education of doctors, health personnel, families of diabetics and people on the signs of diabetes in children to reduce the frequency and severity of pediatric $\mathrm{CDAB}>$

\section{EPO-084 Diabetes and Insulin \\ Acute complication in children receiving intensive treatment for type 1 diabetes

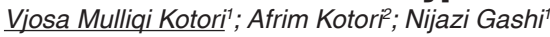 \\ 1 Pediatric Clinic University Clinical Centre, Endocrinology, Prishtina, \\ Kosovo; '2Laboratoiry Diagnostics-LiOri, Clinical Biochemistry, \\ Prishtina, Kosovo}

Introduction: Diabetes type 1 is a chronic metabolic disorder that affects children of all ages. Existing condition in Kosovo, low social economic state, stressed life and lack of organization of health services are unfavorable factors to allow good managing of type 1 diabetes.

Objectives: To assess the effectiveness of intensive treatment with multiple daily injection in children with type 1 diabetes on glycosylated hemoglobin (HbAlc) and episodes of severe hypoglycemia in young children with type 1 diabetes.

Methods: In the study were included patients with type 1 diabetes in intensive treatment aged 4-18 years. All participants underwent education training and introduction to a dietary freedom.

Results: Data were collected from 62 patients in intensive treatment with multiple daily injections, age 4-18 years, 34 female, for 6 months after intensive treatment of diabetes. Diabetes duration ranges from 0-8 years. Recurrent episodes of hypoglycemia were mainly observed in youngest age group (aged 4-6 years), especially from the rural area. The value of $\mathrm{HbAlc}$ ranges from 6.6-8.5. Severe hypoglycemic events were increased from 9 patients in the six months before intensified treatment of diabetes to 23 patients six months after intensified treatment. Mainly episodes of hypoglycemia were observed in children of youngest age 4-6 years due to unpredictable habits of eating. Three out of 23 patients with a history of severe hypoglycemia experienced seizures. Missing glucagons kit make parents and caregivers unable to manage severe hypoglycemia in time.

Conclusion: Intensive treatment and education of parents and children is beneficial in achieving a good glycemia control. Prevention of hypoglycemic episodes needs continues vigilance and education of parents, children, teachers and caregivers for signs of hypoglycemia and controlled meal size. We highlight the application of new insulin analogs and continuous subcutaneous insulin infusion in our country Kosovo as a solution for reduction of hypoglycemic episodes in children with type 1 diabetes.

\section{EPO-085 Endocrine Oncology \\ Suprasellar germinoma with severe hypernatremia and hypopituitarysm in an adipsic child}

Ionela Pascanu'; Rudolf Ruff'; Claudia Banescu; Carmen Duicu; Raluca Petrit

'University of Medicine and Pharmacy, Endocrinology, Targu Mures, Romania; ${ }^{2}$ University of Medicine and Pharmacy, Genetics, Targu Mures, Romania; ${ }^{3}$ University of Medicine and Pharmacy, Paediatrics, Targu Mures, Romania

Background: Germinoma represents $7.8 \%$ of cerebral tumors in pediatric age. Occurrence of diabetes insipidus is common in the case of suprasellar localization.

Aim: To report a case of a 17 year old boy with particularly long term evolution from the onset of symptoms to the positive diagnosis, due to misleading neuroimaging.

Patient: At 9 years he was diagnosed with idiopathic central diabetes insipidus and started desmopressin therapy. In the subsequent years he presented central hypothyroidism and GH deficiency. At 16 years he was referred to our institute with relatively good general condition despite an extremely severe hypernatremia of $189 \mathrm{mEq} / \mathrm{l}$, serum osmolality of $436 \mathrm{mOsm} / \mathrm{kg}$ and impaired thirst sensation, denoting a long lasting osmoregulatory dysfunction. He developed sinus thrombosis as a consequence of hyperosmolality and presented intermittent rises of temperature, probably due to thermoregulatory disorder. During a long term follow-up no organic lesion except for a thickening of the pituitary stalk has been described on neuroimaging. Finally two supracentimetric tumor nodules were shown on contrast enhanced MRI in the suprasellar region and at the floor of the left lateral ventricle. Stereotactic biopsy concluded a pure germinoma, and craniospinal irradiation was performed. At present he has an infracentimetric suprasellar tumor remnant, reperfused sinus veins, optochismatic syndrome sequelae. He benefits from L-Thyroxin and testosterone replacement, and maintains hydro-electrolytic balance on desmopressin and controlled oral fluid intake.

Discussions: Our patients developed marked hypernatremia probably due to damages of both the osmoregulation and thirst centers. This case underlines the difficulties in establishing the etiology of diabetes insipidus and the particularities of hypothalamic disturbances associated with a germinoma.

Conclusions: In supasellar germinoma at the time of diabetes insipidus diagnosis the cerebral mass might be too small to be identified by MRI. In such patients, repeating imaging study should be obtained.

\section{EPO-086 Endocrine Oncology \\ Bad compliance to hypothyroidism treatment and development of papillary carcinoma in adolescent girl}

Gianluca Tornese $^{1}$; Elena Faleschini; ; Caterina Bertolini ${ }^{3}$, Giorgio Tonini ${ }^{3}$

IInstitute for Maternal and Child Health IRCCS "Burlo Garofolo" and University of Trieste, Department of Paediatrics, Trieste, Italy; "Institute for Maternal and Child Health IRCCS "Burlo Garofolo", Department of Paediatrics, Trieste, Italy; ${ }^{3}$ Institute for Maternal and Child Health IRCCS, Department of Paediatrics, Trieste, Italy

Background: A number of studies have suggested that TSH may play a central role in the development and/or progression of thyroid carcinomas Hypotheses: A bad compliance to treatment of hypothryroidism, leading to higher concentration of TSH could lead to development thyroid cancer Case report: We describe the case of a girl who was first diagnosed with hypothyroidism due to Hashimoto thyroiditis when she was 12 . Symptoms begun when she was 8 , but there was a gross delay in diagnosis, leading to a TSH-secerning adenoma at diagnosis, with a secondary GH deficiency who needed treatment with rhGH for 4 years. Compliance to treatment with levothyroxine was bad over the years, leading to frequent finding of TSH $>50$ $\mathrm{mcUI} / \mathrm{ml}$ and up to $100 \mathrm{mcUI} / \mathrm{ml}$ with low FT4 and normalized MRI. When she was 18 , the yearly ultrasound showed in the the right lobe a small altera- 
tion of about $6 \mathrm{~mm}$, with millimetrical punctiform hyperechogenicity referable to microcalcifications which has never seen before. The fine needle aspiration showed a Tir4 citology (probably malignant). A total thyroidectomy was performed and the diagnosis of papillary carcinoma was confirmed with no lymph node metastasis.

Conclusions: The prevalence of thyroid cancer in juvanile autoimmine thyroiditis is $3.0 \%$ and the lack of response to levothyroxine therapy can suggest malignancy. In this case, we believe that bad compliance to treatment with high TSH could have lead to malignant trasformation. Therefore great attention should be paid to compliance to levothyroixe treatment, especially during adolescence.

\section{EPO-087 Endocrine Oncology \\ Bilateral phaeochromocytoma as first manifestation of von Hippel-Lindau disease: a case report \\ Gonul Catli'; Ayhan Abaci'; H. C. Hartmut Neumann²; Ayca Altincik' Korcan Demir'; Ece Bober \\ 'Dokuz Eylul University, Pediatric Endocrinology, Izmir, Turkey; \\ ${ }^{2}$ Albert-Ludwigs-University, University Medical Center, Nephrology \\ Department, Freiburg, Germany}

Background: Von Hippel-Lindau syndrome is an autosomal dominant disorder which includes susceptibility to hemangioblastomas of the eyes and central nervous system, renal clear cell carcinoma, multiple pancreatic cysts, serous cystadenomas and pancreatic neuroendocrine tumors, pheochromocytoma, endolymphatic sac tumours and cystadenomas of epididymis and broad ligament.

Objective and hypotheses: To present the clinical features and genetic analyses of a boy and his mother with Von Hippel Lindau disease.

Methods: A 10-year-old boy was admitted to our hospital with complaints of headache and diplopia. His brachial blood pressure was 150/120 mmHg. His mother had been operated because of bilateral pheochromocytoma at the age of twenty-three.

Results: Laboratory tests showed elevated 24-h urinary vanillylmandelic acid and normetanephrine but normal metanephrine levels. Abdominal MRI indicated the presence of multiple lesions in paravascular areas neighboring both adrenal glands. After bilateral subtotal adrenalectomy the diagnosis of bilateral pheochromocytoma was confirmed histologically. The patient remained asymptomatic with no laboratory and radiologic abnormalities for six years of follow-up. At the age of 16 he referred to our clinic with bilateral retinal hemangiomas. Positive family history with bilateral pheochromocytoma and retinal hemangioma revealed the diagnosis of VHL disease. Cerebral, spinal MRI and abdominal CT were normal. Mutation analysis of the patient and his mother indicated a mutation in exon 3 of the VHL gene (c. $695 \mathrm{G}>\mathrm{A}$, aminoacid p.R161Q). The patient's mother was evaluated by appropriate imaging techniques and no VHL tumors was detected.

Conclusions: In children phaeochromocytoma may be the only and/or initial manifestation of the disease with delayed manifestations of the syndrome in other organs. Molecular analysis of the VHL gene is useful for early diagnosis of the disease in individuals who do not yet fulfill the clinical diagnostic criteria and is instrumental for the management and follow-up of the affected family.

\section{EPO-088 Endocrine Oncology}

\section{Nephrolithiasis as a manifestation of primary hyperparathyroidism}

Carla Costa ${ }^{1}$; Janine Coelho ${ }^{2}$; Américo Gonçalves²; Clara Pereira²; Glória Silva'; Helena Pinto ${ }^{3}$; Ana Teixeira ${ }^{3}$; Carlos Mariz'; Cíntia Catro-Correia ${ }^{1}$; Manuel Fontoura

${ }^{1}$ Hospital São João, Paediatric/ Paediatric Endocrinology Unit/ Faculty of Medicine of Porto, Porto, Portugal; ${ }^{2}$ Hospital São João, Paediatric, Porto, Portugal; ${ }^{3}$ Hospital São João, Paediatric/ Paediatric Nephrology Unit / Faculty of Medicine of Porto, Porto, Portugal; ${ }^{4}$ Hospital São João, Paediatric Surgery, Porto, Portugal

Background: The primary hyperparathyroidism (PHPT) is a rare condition in adolescents. Nephrolithiasis appears in $15-20 \%$ of these patients. The PHPT could be the first manifestation of multiple endocrine neoplasia (MEN) type 1 and type $2 \mathrm{~A}$.
Clinical case: Fourteen year old male adolescent with a past history of nephrolithiasis diagnosed in January 2011. In the family history it should be highlighted the father's history of nephrolithiasis. During the study of nephrolithiasis was identified hypercalcaemia, high levels of parathyroid hormone (PTH), a low level of 25-OH-vitamin D and hypercalciuria. Physical examination: normal. The bone mineral density showed osteopenia in the lumbar spine (Tscore -2,3). A cervical ultrasound was performed which revealed normal thyroid with the presence of a nodular structure in the left lobe. The TC-99m sestamibi scan showed a focus of hyperfixation at post-inferior side of the left thyroid lobe, suggestive of abnormal parathyroid, and another one at retro-external location that could translate intra-thymus parathyroid. The definitive diagnostic was parathyroid adenoma with symptomatic PHPT. The patient was thereby submitted to parathyroidectomy of the gland affected by the adenoma and partial excision of thymus, corresponding to the image in the scan. After surgery, the blood exams showed normalization of serum calcium and PTH. Was requested a genetic study to detect MEN1 and MEN2A.

Conclusions: The parathyroid adenomas represent approximately $89 \%$ of the PHPT. The intra-thymic parathyroid adenomas express a particular gene (GCMB) suggesting their origin in parathyroid cells. Vitamin D deficiency is common in patients with PHPT. However, the replacement with vitamin D remains controversial, because it can have a worse effect on hypercalcaemia and hypercalciuria. Surgical treatment remains the first choice in cases of symptomatic disease. Since this adolescent may develop future recurrence of PHPT, which may be associated with MEN1 and MEN2A, the authors emphasize the need to maintain an appropriate follow-up.

\section{EPO-089 Fat Metabolism, Obesity \\ The prevalence and risk factors of obesity among adolescents in Ankara, Turkey}

Sírma Ercan'; Yíldíz Bilge Dallar'; Serdar Onen'; Engiz Özlem $^{2}$

${ }^{1}$ Ankara Training and Research Hospital, Pediatrics, Ankara, Turkey;

${ }^{2}$ Ankara Training and Research Hospital, Pediatric Endocrinology,

Ankara, Turkey

Background: The prevalence of childhood obesity is on the rise around the world. The World Health Organization has named obesity a major public health problem in 1998. Childhood and adolescence obesity is related to an increased adult morbidity and mortality by leading to a variety of conditions such as diabetes mellitus, hypertension, psychological disorders and social problems. Therefore, childhood obesity should be identified and treated promptly.

Objective and hypotheses: The purpose of this study was to examine the prevalence and the risk factors associated with obesity among adolescents in Ankara, Turkey.

Methods: The study was designed as a cross-sectional, population based study. A representative sample of children in Ankara was randomly selected. Body mass index (BMI) of 8848 children aged 11-18 years was compared with the BMI references for Turkish children to estimate the prevalence of obesity. A standardized questionnaire which determines sociodemographic characteristics, computer usage, television watching, physical activity and the presence of obesity in the family was applied to the study group. Data were analyzed with SPSS for Windows 11.5 package program. $\mathrm{P}<0.05$ was considered statistically significant.

Results: The results showed that the overall prevalence of obesity among adolescents was $7.7 \%$ ( $8.4 \%$ for females and $7.0 \%$ for males). The study showed that computer use increased as BMI increased. Overweight group and obese group watched TV and used computer for $\geq 2$ hours/day more compared to normal weight group. Normal weight group engaged more in regular physical activity. Obesity prevalence in the family of obese adolescent was $56.5 \%$. Conclusions: The prevalence of childhood obesity in Ankara, Turkey is lower than European countries and US. Computer use, watching television, physical activity and family factors are important risk factors for obesity. 


\section{EPO-090 Fat Metabolism, Obesity \\ New risk factors for coronary artery disease in obese adolescents compared with controls - a case control study \\ Rahim Vakili'; Mohsen Namaty² Mohamad Safarian²; \\ Majid Ghayour-Mobarhan²; Samaneh Kouzehgaran'; \\ Mohamad Khajedaluee \\ ${ }^{1}$ Mashhad University of Medical Sciences, Pediatric Endocrinology, \\ Mashhad, Islamic Republic of Iran; ${ }^{2}$ Mashhad University of Medical \\ Sciences, Nutrition, Mashhad, Islamic Republic of Iran; ${ }^{3}$ Mashhad \\ University of Medical Sciences, Social Medicine, Mashhad, Islamic \\ Republic of Iran}

Background: Coronary atery diseases are considered as the most common cause of death in all societies including Iran. Considering the changes life style, reduced mobility, increased consumption of foods with high fat and increased growth of obesity among children and adolescents.

Objective and hypotheses: This study seeks to compare the new risk factors of coronary-artery diseases in obese adolescent with the control group.

Methods: In this case control study, from the obese adolescents registered in the nutrition clinic of Ghaem Hospital, 80 were selected as well as the 80 adolescent students having the same gender and age as the previous one but with normal weight. These two groups were selected randomly and their serum level of vitamin D, 27 anti-heat shock protein (HSPs), the balance of oxidants and antioxidants and homocysteine were determined and compared using kits adopted from Sigma and Merk companies. The data were analyzed statistically in significant level of $\mathrm{p}<0.05$ using SPSS15, Chi-square Test, Mann- Whitney and Independent $\mathrm{T}$ tests.

Results: $42(52.5 \%)$ of the subjects in the first group and $44(55 \%)$ of subjects in the control group were female. There was no significant difference between the mean of vitamin D, homosysteine, anti-hsp27, oxidants and antioxidants in these two groups as was the mean serum levels of HDL and LDL. However, the mean of TG and Cholestrol in obese group was significantly higher than that of control group: $(\mathrm{p}<0.05)$

Conclusions: Since researches have diagnosed obesity and high levels of homocysteine and TG as the major risk factors of cardiovascular disease as well as the onset of metabolic diseases such as diabetes, future studies need to examine these factors in some other cities and countries to help prevention of coronary artery disease and its expeditious diagnosis

\section{EPO-091 Fat Metabolism, Obesity \\ Obesity epidemiology in children living in the lower socio-economic status \\ Enver Simsek ${ }^{1}$; Beray Selver ${ }^{2}$; Yildiz Dallar ${ }^{2}$; Cigdem Binay'; Cengiz Ba/ ${ }^{3}$ \\ ${ }^{1}$ Eskisehir Osmangazi University School of Medicine, Pediatric Endocrinology, Eskisehir, Turkey; ${ }^{2}$ Ankara Research and Training Hospital, Pediatrics, Ankara, Turkey; ${ }^{3}$ Eskisehir Osmangazi University School of Medicine, Biostatistics, Eskisehir, Turkey}

Background: Although obesity is considered as an epidemic health problem in then worldwide, the implication of socio-economic status (SES) in these health conditions have rarely been investigated.

Methods: This is cross-sectional study was carried out between May 2009 and August 2011 in children. Inclusion criteria to the study were have a slum life and to be in lower monthly income category according to the economic condition of Turkey. We randomly enrolled 80 obese boys $(11.1 \pm 2.7$ years old) and 121 obese girls (11.4 \pm 2.9 years old). The height and weight data were used to calculate the BMI $(\mathrm{kg} / \mathrm{m} 2)$ using the formula: weight $(\mathrm{kg})$ divided by height $(\mathrm{m})$ squared. International cutoff points were applied to define obesity1. In obese children, daily exercise programs, the usage of school buses, daily TV watching, computer-game playing, and fast-food consuming trends were questioned. In addition, Whether or not obesity in mother and father, the mother's and father's education level, monthly income, family history of hypertension, type 2 diabetes mellitus and heart attack in the family and their first-degree relatives were questioned. Table 1 shows the comoarisons epidemiological factors between obese and control subjects living in the lower socio-economical conditions. Table 2 shows logistic regression analysis on epidemiological parameters for obesity.

Conclusion: This study revealed that, computer game playing, predisposition to consumption fast-food and carbonated drinks are important risk factors for obesity in living lower socio-economical condition, as known that all of these factors are obesity risk factor in middle- or higher socio-economical conditions. To be house women is additional risk factors for obesity. Living in apartment, has an obese father, type 2 diabetes mellitus and heart attack history are predictive risk factor for obesity living in the lower socio-economical condition.

\begin{tabular}{lccc} 
& $\begin{array}{c}\text { Obese } \\
(\mathbf{n}=201)\end{array}$ & $\begin{array}{c}\text { Control } \\
(\mathbf{n}=38)\end{array}$ & $\mathbf{p}$ \\
\hline Computergame playing (yes-no) & $127-74$ & $15-23$ & $<0.05$ \\
\hline Using school bus (yes-no) & $31-170$ & $6-32$ & $>0.05$ \\
\hline Predisposition to consumption of fast food (yes-no) & $146-55$ & $18-20$ & $<0.001$ \\
\hline Predisposition to carbonated drinks (yes-no) & $172-29$ & $27-11$ & $<0.05$ \\
\hline Does the mother has a job? (yes-no) & $32-169$ & $0-38$ & $<0.05$ \\
\hline Does the mother has obesity? (yes-no) & $88-113$ & $10-28$ & $>0.05$ \\
\hline Does the father has obesity? (yes-no) & $65-134$ & $5-33$ & $<0.05$ \\
\hline Heart attack history in family (yes-no) & $55-144$ & $3-35$ & $<0.05$ \\
\hline Type 2 diabetes mellitus history in family (yes-no) & $128-73$ & $10-28$ & $<0.001$ \\
\hline Slum life or an apartment life & $72-179$ & $23-15$ & $<0.05$ \\
\hline $\begin{array}{l}\text { Mother's education level; primary school - secondary } \\
\text { school or university }\end{array}$ & $156-45$ & $35-3$ & $>0.05$
\end{tabular}

\begin{tabular}{lcccc} 
Variable & $\boldsymbol{\beta}$ & $\mathbf{p}$ & $\begin{array}{c}\text { Odd Ratio } \\
\text { (OR) }\end{array}$ & $\begin{array}{c}95 \% \text { Confidendal } \\
\text { Interval (C.I.) }\end{array}$ \\
\hline Computergame playing & 0.852 & 0.052 & 2.34 & $0.99-5.44$ \\
Obese father & 1.368 & $<0.001$ & 5.08 & $1.54-16.75$ \\
\hline Heart attack history in family & 1.358 & 0.54 & 3.89 & $0.97-15-49$ \\
\hline Type 2 Diabetes Mellitus history in family & 1.911 & $<0.001$ & 6.76 & $2.54-17.97$ \\
Slum life & 1.90 & $<0.001$ & 6.68 & $2.41-18.54$
\end{tabular}

\section{EPO-092 Fat Metabolism, Obesity \\ Serum levels of uric acid correlate with inflammation markers in prebuberal obese children \\ Ramón Cañete ${ }^{1}$; Miguel Valle²; Rosario Martos ${ }^{3}$; Rosario Valle4; $M^{a}$ Dolores Cañete ${ }^{5} M^{a}$ del Mar Urbano 6 ; Eva L Van Donhelar ${ }^{7}$ ${ }^{1}$ Hospital Universitario Reina Sofía, Pediatric Endocrinology Unit, Cordoba, Spain; ${ }^{2 H}$ ospital Valle de los Pedroches, Clinic Laboratory, Pozoblanco, Córdoba, Spain; ${ }^{3}$ Centro de Salud, Pediatria, Pozoblanco, Córdoba, Spain; ${ }^{4}$ Faculty of Medicine, Pediatria, Córdoba, Spain; ${ }^{5}$ University of Córdoba, Grupo PAIDI CTS-329. IMIBIC, Cordoba, Spain; ${ }^{6}$ Hospital Valle de los Pedroches, Clinic Laboratory, Cordoba, Spain; ${ }^{7}$ Maastricht University, Faculty of Health, Medicine and Life Sciences, Maastricht, Netherlands}

Background: Association between an increase in uric acid (UA) and various inflammation markers, such as C-reactive protein (CRP) and interleukin-6 (IL-6) can begin at early age in obese children. Studies that evaluate this possible association are limited, especially at prepuberal age.

Objectives: To evaluate the possible differences in UA levels and inflammation markers between prepuberal obese and non obese children, and determine whether there is a relation between UA levels and inflammation markers. Methods: A transversal study was carried out in prepuberal children (6-9 years old). 43 obese children were included and the same number of non obese children as controls, matched by age and sex. Parameters determined for all of them: antropometric parameters, serum levels of UA, CRP and IL-6. Results: Mean age in the obese children group was $8.03 \pm 0.17$ years and 7.91 \pm 0.15 for the control group. In the obese group, body mass index (BMI, $\mathrm{kg}$ / $\mathrm{m} 2$ ) was $23.68 \pm 0.34$ and $16.83 \pm 0.21$ for the non obese children. Concentrations of UA $(p=0.006)$ and CRP $(p<0.001)$ were significantly higher in obese children. In the obese group, UA levels showed a positive correlation with BMI $(r=0.393 ; p<0.01)$, CRP $(r=0.338 ; p<0,05)$ and IL-6 $(0.361 ; p<0.05)$. Corrected for age and sex, UA levels are an independent predicting factor for CRP and IL-6 concentrations.

Conclusions: UA levels and inflammation markers are elevated in prepuberal obese children. There is an association between UA and inflammation variables.

Horm Res 2012;78(supp1 1) 
EP0-093 Fat Metabolism, Obesity

Nonalcoholic fatty liver disease in childhood and plasmatic leptin

$M^{a}$ Dolores Martínez-Jiménez'; Mercedes Gil-Campos²; Ángel Gil-Hernández; ${ }^{3}$ Concepción $M^{a}$ Aguilera $^{3}$; Ramon Cañete

${ }^{1}$ Hospital General de Ciudad Real, Pediatrics Unit, Ciudad real, Spain;

${ }^{2}$ Hospital Universitario Reina Sofía, Pediatrics Unit, Córdoba, Spain;

${ }^{3}$ Universidad de Granada, Department of Biochemistry and Molecular

Biology 2, Granada, Spain; ${ }^{4}$ Hospital Universitario Reina Sofía.,

Pediatrics Unit, Córdoba, Spain

Background: Nonalcoholic fatty liver disease (NAFLD) in infants has increased in the last decades in line with obesity. Additionally to obesity, insulin resistance (IR) and hyperlipemia are considered as risk factors for NAFLD, with leptin role being controversial. The objective of the study is to evaluate known risk factors for NAFLD and their possible association with leptin during pediatric age.

Materials and methods: A prospective descriptive study was carried out on 382 prepuberal children (167 obese (OB), 72 overweight (OW) and 143 controls (C)). IR was evaluated (using HOMA index), and also levels of plasmatic cholesterol and its fractions (HDL and LDL), triglycerides (Tg), alanine transaminase (ALT) and leptin. On a subsample of 60 patients (48 OB and $22 \mathrm{C}$ ) an hepatic echography was performed to search for signs of NAFLD. Afterwards, statistic correlations between ALT and the rest of the parameters analyzed were evaluated.

Results: OB children showed higher levels of IR, Tg, ALT and leptin, and lower levels of cholesterol and HDL than the other groups. NAFLD in infants, represented by ALT, correlated with body mass index (BMI) (r: 0.40), HOMA index (r: 0.27 ) and leptin ( $\mathrm{r}: 0.23$ ) independently from obesity $(\mathrm{p}<0.001)$, but not with $\mathrm{Tg}$. Hepatic echography was pathological in a similar proportion in both groups (OB 19.3\% vs C 18.9\%) and with normal mean ALT values. Conclusions: Obesity and IR may be considered as risk factors for NAFLD in the prepuberal stage. The fact that plasma leptin correlates with ALT might support the contribution of this adipokine to the development and progression of NAFLD.

\section{EPO-094 Fat Metabolism, Obesity}

\section{Changes in short and medium term in body composition in children in a educational and integral program for obesity}

Ignacio Diez-Lopez'; Ainhoa Sarasua'; Isabel Lorente'; Victor Rodriguez-Rivera²; Amaia Antón-Perales²;

Garbiñe Amunarriz-Aizpuru²; Maria Guadalupe Castaño-Martinez²; M.Puy Portillo-Baquedano ${ }^{2}$ :M.Teresa Macarulla-Arenaza ${ }^{2}$ 'University Hospital of Alava, Peadiatric Endocrinology Unit, Vitoria, Spain; ${ }^{2}$ Basque Country University, Nutrition and Obesity Group, School of Pharmacy, Vitoria, Spain

Introduction: Childhood obesity is a growing problem especially in Spain, European Union, which has the highest prevalence. It is therefore necessary to implement effective preventive and treatment.

Objective: To evaluate the effectiveness in the short to medium term, in terms of body composition, of a comprehensive educational program and group with parental involvement.

Methods: We recruited 40 patients ( 25 girls and 15 boys) between 8 and 13 years are overweight or obese. They and their parents were included in the re-education program of behavioral "Nens in movement" of the Hospital Val d'Hebron in Barcelona. Before starting the program to an end and 6 months later, he determined the weight, height, the waist and hip circumferences and body composition by bioelectrical impedance multichannel, and calculated the body mass index $(\mathrm{BMI}=$ weight $/$ stature2), Body Fat Index $(\mathrm{BFI}=\mathrm{fat}$ mass/stature2), the Lean Body Mass Index (LBMI = No fat mass/stature2) and waist / hip ratio (WHR). The data were analyzed using the Student test for paired samples $t$ with a confidence interval of $95 \%$.

Results: At the end of the program, and only one drop, $87 \%$ of patients had reduced their BMI, $79 \%$ the BFI and the WHR $82 \%$ and $26 \%$ had increased LBMI. Six months later, 74\% continued to maintain a lower BMI, $77 \%$ a lower BFI and $74 \%$ a lower WHR and $41 \%$ had increased LBMI. Regarding gender differences, girls showed better results in BFI and BMI; and boys were best in I WHR and LBMI. The mean values of all the patients are summarized in the table:

\begin{tabular}{lcccc|}
$\begin{array}{l}\text { * Values significantly different } \\
\text { from baseline }(\mathbf{P}<0.05) .\end{array}$ & $\begin{array}{c}\text { Stature } \\
(\mathbf{c m})\end{array}$ & $\begin{array}{c}\text { Weight } \\
(\mathbf{k g})\end{array}$ & $\begin{array}{c}\text { Fat } \\
(\%)\end{array}$ & $\begin{array}{c}\text { No fat mass } \\
(\%)\end{array}$ \\
\hline Male Female & Male Female & Male Female & Male Female \\
\hline At the begginng program & 148148 & $57,358,7$ & $33,537,8$ & $66,562,2$ \\
\hline At the end program & $151^{*} 150^{*}$ & $57,758,8$ & $31,9^{*} 36,6^{*}$ & $68,1^{*} 63,4^{*}$ \\
\hline 6 mouths later & $152^{*} 152^{*}$ & $60,4^{*} 60,4^{*}$ & $31,8^{*} 34,7^{*}$ & $68,2^{*} 65,3^{*}$
\end{tabular}

Conclusion: The program is effective in the short and medium term. Although the medium term weight increase appears to indicate a loss of effectiveness, it should be borne in mind that most of it was due to an increase in lean body mass and fat.

\section{EPO-095 Fat Metabolism, Obesity \\ The relationship of bone, fat tissue and glucose metabolism in children with obesity: is there any association between osteocalcin, adiponectin and metabolic syndrome?}

Nilay Abseyi; Zeynep Siklar; Merih Berberoglu; Bulent Hacihamdioglu; Senay Savas Erdeve; Gönül Öcal

Ankara University School of Medicine, Pediatric Endocrinology, Ankara, Turkey

Background: In recent years in particular, scientific interest has focused on the association between fat tissue, skeletal system and glucose metabolism. There are few studies investigating the association between osteocalcin and insulin resistance.

Objective and hypotheses: The aim of the present study was to assess the effects of adiponectin which is protective of metabolic syndrome and osteocalcin on the development of metabolic syndrome and insulin resistance in obese children.

Methods: Children and adolescents between 5 and 18 years of age, in whom obesity, were included in the study. In addition to the routine laboratory analyses, adiponectin and osteocalcin levels were also analyzed. The association between the components of metabolic syndrome and adiponectin and osteocalcin levels was investigated.

Results: Insulin resistance was identified in 34\% and metabolic syndrome was identified in $22 \%$ of the cases. Osteocalcin levels were significantly lower in girls than boys $(69.63 \pm 36.83$ vs. $95.69 \pm 38.18)$. An inverse association was found between age and osteocalcin levels. No significant association was found between osteocalcin levels and metabolic syndrome parameters [fasting blood glucose, fasting insulin, triglyceride, total cholesterol, LDL-C, HDL-C, glucose/insulin ratio and the homeostasis model assessment insulin resistance index (HOMA-IR)]. Adiponectin levels were significantly low in cases with metabolic syndrome and in the presence of dyslipidemia. An inverse association was found between triglyceride levels, fasting insulin levels and adiponectin. No significant association was found between adiponectin and osteocalcin levels.

Conclusions: Adiponectin levels were low in obese children with metabolic syndrome and were associated with insulin resistance and the other components of metabolic syndrome as expected. However, no direct association was found between osteocalcin and insulin resistance or metabolic syndrome. No findings indicating the interaction between osteocalcin and adiponectin in the development of metabolic syndrome could be identified.

\section{EP0-096 Fat Metabolism, Obesity}

\section{Serum apelin levels in male obese children and correlation with insulin resistance}

Hongshan Chen; Hongjun Ba; Zhe Su

The First Affiliated Hospital of Sun Yat-sen University of Medical

Sciences, Paediatric Department, Guangzhou, China

Objective and hypotheses: To investigate serum apelin concentrations in male obese children and its correlations with body mass index (BMI), and insulin sensitivity.

Methods: Twenty-eight male obese children, aged (10.91 \pm 2.08$)$ years old, and 25 male controls, age $(11.01 \pm 2.35)$ years old, were enrolled in the study. Serum levels of apelin, fasting glucose and fasting insulin (FINS) were measured. Body mass index and insulin resistance index (HOMA-IR) were calculated for all participants. 
Results: There was a significant difference in BMI between the obese and normal subjects $(26.72 \pm 4.03$ Vs $16.46 \pm 1.78, \mathrm{p}<0.001)$. The obese subjects had significant higher FINS [22.1(14.95 39.42) Vs 6.5(5.1 8.4), $\mathrm{p}<0.001]$ and HOMA-IR [4.90 (3.46 8.18) Vs $1.46(1.16 \sim 2.1), \mathrm{p}<0.001]$ than nonobese ones. There were no sstatisticallly significant difference of the serum apelin concentrations between the male obese group and that of the male controls [1.05 ( 0.77 1.25) Vs 1.12(0.88 1.57), p>0.05]. Serum apelin level was not correlated with HOMA-IR.

Conclusions: No significant difference was found for the serum apelin concentrations between the male obese group and that of the male controls. Further investigation is needed to clarify the role of the apelin in Metabolic Syndrome.

\section{EP0-097 Fat Metabolism, Obesity \\ Influence of anthropometrical and mental statuses on occurrence of behaviour diseases among children of school age \\ Angelika Solntseva; Katsiaryna Lepiatsila \\ Belarussian State Medical University, Pediatrics, Minsk, Belarus}

Objective and hypotheses: The aim of this study was to estimate influence of the anthropometrical status and personal features on occurrence of psychosomatic frustration, in particular, eating disorder, among children of school age. Methods: We studied 57 children (age 15 \pm 1 ,3years). They were interviewed with Toronto Alexithimia Scale (TAS), scale of Rotter, standartized questionnaire "Aim-Means-Result" (AMR), scale of Rean and divided into 3 groups on the basis of body mass index (BMI). 1) Group A (BMI $<19 \mathrm{~kg} / \mathrm{m} 2,2)$ Group B $(19 \mathrm{~kg} / \mathrm{m} 2<$ BMI $<24 \mathrm{KG}$ (BMI C 3)Group m2), $>24 \mathrm{~kg} / \mathrm{m} 2)$. A statistical analysis was perfomed.

Results: Based on the TAS $41 \%$ of boys showed higher than normal rates of alexithymia ( Group A - 40\%, Group B - 60\%, Group C - 100\%). Among girls $(75 \%, 50 \%, 43 \%$ respectively). AMR: $87 \%$ b and $82 \%$ g can't substantiate their goals. $50 \%$ of children had psychological barriers. Low-energy potential was recorded in Groups A and C (17\% and 25\%). 66\% girls and 88\%boys of all groups indicated optimal results. Scale of Rotter. Boys of Group C were dominated by external locus of control. Girls of such group were dominated by internal locus of control of life. Motivational pole is not strongly marked in $61 \%$ of girls.

Conclusions: The analysis of TAS showed an increasing trend of alexithymia among boys (from $59 \%$ to $100 \%$ ) and lower among girls (from $75 \%$ to $43 \%$ ) with an increase in BMI. Boys with a BMI $>24 \mathrm{~kg} / \mathrm{m} 2$ prevailed external locus of control, the girls - internal locus of control. In analyzing the results of the questionnaire of Rean boys tended to reduce the motivation to succeed and grow with the increase of negative motivation with increasing BMI (success from $70 \%$ to $25 \%$ ).

\section{EPO-098 Fat Metabolism, Obesity \\ The assessment of early microvascular aging and cardiovascular performance in obese adolescents \\ Bulent Hacihamdioglu'; ; Gonul Ocal'; Merih Berberoglu'; \\ Zeynep Siklar'; Ercan Tutar'; Gokhan Nergizoglu; \\ Senay Erdeve Savas'; Emine Camtosun \\ ${ }^{1}$ Ankara University Faculty of Medicine, Departments of Pediatric \\ Endocrinology, Ankara, Turkey; ${ }^{2}$ Ankara University Faculty of Medicine, Departments of Pediatric Cardiology, Ankara, Turkey; ${ }^{3}$ Ankara \\ University Faculty of Medicine, Internal Medicine, Nephrology, Ankara, Turkey}

Background: Adverse effects on cardiovascular system of obesity in adults are well known.

Objective and hypotheses: Based on the hypothesis that there may be adversely affected cardiovascular performance even in adolescent period, in this study it was aimed that to evaluate early microvascular aging and cardiovascular performance in the obese adolescents.

Methods: Total 61 adolescent obese (29 mildly obese, 32 serious obese) were evaluated in this study. We were aimed to reveal the potential cardiovascular exposure in the obese adolescents by arterial tonometry and treadmill test and to screen the cardiovascular risk factors. Cardiovascular risk factors; hypertension, dyslipidaemia, impaired glucose metabolism; abdominal obesity, intra-abdominal adipose tissue distribution, hepatosteatosis and chronic inflammation were aimed to control. Control group was included in the study to compare parameters of the arterial tonometry (n: 58$)$.

Results: Maximum exercise time was not showed difference associated with the degree of obesity or the presence of the metabolic syndrome. We evaluated arterial stiffness by arterial tonometry in this study, and we found that; pulse wave velocity in obese adolescents was significantly higher than the control group in the same age $(5.0 \pm 0.71$ versus $4.7 \pm 0.5, \mathrm{p}<0.05)$. Preperitoneal adipose tissue thickness was the single variable which affecting the pulse wave velocity on the regression analysis (Beta: $0.325, \mathrm{p}<0.05$ ).

Conclusions: Early vascular aging due to obesity may begin in the adolescent period and this can be detected by increased pulse wave velocity. Preperitoneal adipose tissue thickness is an important parameter which associated with increased pulse wave velocity. Preperitoneal adipose tissue appears to be associated with the risk of cardiovascular disease like visceral adipose tissue. Prospective, controlled studies are needed for better understanding of the relationship between the preperitoneal adipose tissue and the cardiovascular risk factors.

\section{EP0-099 Fat Metabolism, Obesity \\ The influence of parent's BMI, educational level and family structure on obesity prevalence in prepubrtal-aged children}

Angelica Solntsava; Liudmila Viazava; Olga Zagrebaeva

Belarusian State Medical University, pediatrics, Minsk, Belarus

Background: Lifestyle of the family seems to play an important role in the nutritional and behaviors choices of their children.

Objective and hypotheses: The aim of this study was to analyse the relationships between prepubertal-aged children's weight status, parents' educational level and other indicators of the socio-economic status of families in Belarus. Methods: The children samples was selected from national research project "Childhood obesity" and consisted of 217 (129 males and 88 females) prepubertal -aged children. There were measured weight and height, and body mass index (BMI) were calculated and evaluated according to Belarusian population criteria. Parents' weight, height, educational level, family structure and number of children were collected by a self-administered questionnaire. Educational levels were classified as high medium and low. We examined 194 obese (BMI $>95$ percentile for age and sex) children (mean age 7,9 $9 \pm 0,2$ years) and 23 normal weight (BMI $<90$ percentile for age and sex ) children (mean age $4,9 \pm 1,1$ years).

Results: The prevalence of obese children increased along the parents' BMI category: from $22,8 \%$ for normal weight mothers to $34,1 \%$ for obese mothers $(\mathrm{p}=0,005)$ and from $6,8 \%$ for normal weight fathers to $39,5 \%$ for obese fathers $(<0,001)$. An inverse relationship was observed between parents' educational level and child obesity, the lowest educational level conformed to highest prevalence of obese children: $91,9 \%$ for mothers with a low educational level compared to $85,8 \%$ for mothers with a high educational level $(\mathrm{p}<0,001)$; similarly the corresponding prevalence for fathers was $95,0 \%$ to $84,4 \%(\mathrm{p}=0,04)$. The same relationship was noted between child obesity and family structure: $97,6 \%$ in one-parent compared to $86,6 \%$ in two-parent families $(\mathrm{p}<0,001)$. Child weight status was no related to number of children.

Conclusions: Parents' obesity, educational level and family structure, particularly the mother's seem to have an effect on the prevalence of obesity in Belarusian children.

\section{EPO-100 Fat Metabolism, Obesity \\ Causes of failure of weight reduction programs in children \\ Shokery Awadalla \\ Hospital san Jose, Pediatric Endocrinology, Bogota, Colombia}

Background: Failure in weight reduction programs is frequent in obese children. Although it seems easy to eat better and exercise more, weight reduction is a difficult task.

Objective and hypotheses: To clarify the principal causes of this failure we include 200 families with one or more obese children. 235 children, 122 boys and 113 girls with body mass index $>$ percentile 95 were included.

Methods: Age was $11 \pm 0.9$ years. Genetic and hormonal causes were excluded. All the families were instructed about nutritional and exercise recom- 
mendations. Children were evaluated every 2 months for 6 months. Questionnaire about the adherence to the recommendation were realized on each visit. Results: The first 2 month 80 boys $(65 \%)$ and 90 girls (79\%) loosed weight. The second 2 month this number were reduced to 60 boys (49\%) and 72 girls $(63 \%)$. The third 2 months 45 boys (36\%) and 60 girls (53\%) loosed weight. All the rest augmented their weight. Always more girls loosed weight than boys $(\mathrm{p}<0.05)$. Analyzing the data of the questionnaire; father's lack of time was the main reason to abandon physical excursive $(55 \%)$. The second cause was no cooperation of the grandparents offering unhealthy food, as they see the children healthy and not obese (32\%). Other causes $(18 \%)$ were children's refusal to eat the offered healthy food or to make physical exercise, fractures or sickness during the observation period.

Conclusions: Failure to lose weight or incidence in obesity in the majority of cases is responsibility of parents and grandparents. Education programs should address them about the adult risk their children can have.

\section{EPO-101 Fat Metabolism, Obesity \\ Are weight loss intervention programmes for children and adolescents effective in the long term? \\ Amanda Driffield \\ University of Leeds, School of Healthcare, Leeds, United Kingdom}

Background: Childhood obesity is one of the most serious public health challenges of the 21 st century. In the 1990's there was a significant policy response to this statement and many countries set up community based weight management programmes. These programmes were multi-faceted and had a wide variation in content. The published evidence demonstrates their effectiveness but is this maintained in the longer term?

Objective and hypotheses: A systematic literature search was conducted to obtain relevant published articles. All studies were included. A total of 6 papers from differing countries were included, which reported long term outcomes from their interventions, ranging from 1-10 years. Population Children and adolescents ranging between 6-16 years were included. They all participated in a community based weight management programme lasting on average between 8-12 weeks.

Results: Presentation of results is challenging, due to the wide range of types of studies, differing interventions, sample size and heterogeneity. All 6 papers report some positive long term effects, with reduction in BMI. Meta-analysis was not possible due to heterogeneity.

Conclusions: There is some evidence that community based weight management programmes for children and adolescents can continue to be effective in the long term. This is variable and dependent upon the intervention programme. There does seem to be stronger evidence that a family based approach is more effective in the younger child, and these effects are seen in the longer term.

\section{EPO-102 Fat Metabolism, Obesity}

\section{Lipodystrophy and metabolic disease in a patient with atypical progeroid syndrome (APS)} Emanuela Scarano; Federica Tamburrino; Annamaria Perri; Benedetta Vestrucci; Monica Guidetti; Maria Chiara Ragni; Laura Mazzanti

Rare Disease and Auxology Unit, Department of Pediatrics, S.OrsolaMalpighi Hospital, University of Bologna, Bologna, Italy

Background: APS is a new AD progeroid disorder with lipodystrophy and metabolic disease due to LMNA mutation.

Case report: Italian girl with normal psychomotor development. At 8 yrs she developed facial dysmorphic features, type A lipodystrophy and difficulty in walking. Height and weight were normal. At physical examination: prominent eyes, retrognathia, beaked nose, prominent cheeks, high-arched palate, overcrowding of the lower jaw; distal phalangeal hypoplasia of the hands; thin, dry skin with mottled hyperpigmentation, telangiectasias on the trunk; prominent muscles in the lower arms; type A lipodystrophy. At X-ray: hypoplasia of distal fingers of the hands. At the abdominal echography mild hepatic steatosis. Clinical signs were indicative of progeroid syndrome. LMNA gene analysis showed a de novo P4R heterozygous missense mutation and permitted the diagnosis of APS syndrome. After 5 yrs of follow-up, at 14 yrs of age, height and weight were normal. Menarche at 12 yrs with regular men- ses, breast development minimal in size but not atrophic. In the follow-up we noticed a slight increase in micrognathia, type A lipodystrophy with reduction of sc fat, thin skin and mild signs of insulin resistence and glucose intolerance. P4R mutation was reported in a family, transmitted in an AD fashion. Similar to these cases, our patient showed typical facial appearance, type A lipodystrophy, mottled pigmentation and prominent veins of the skin, breast small in size; normal menarche, menses, final height and BMI.

Conclusions: Given the rarity of APS, it is important to recognize the clinical signs in the pediatric age. A precocious diagnosis permits an adequate and inter-disciplinary follow-up to control the evolution of the diseases. An adequate food survey with a personalized diet is important for metabolic disease. In pts with P4R mutation lipodistrophy seems to be precocious and metabolic disease appears in adulthood. Phenotypical signs seem to be milder than in other APS conditions.

\section{EPO-103 Fat Metabolism, Obesity \\ Relationship between percentage of total body fat and metabolic syndrome risk factors in the adolescent}

Peyami Cinaz; Nurullah Celik; Mahmut Orhun Camurdan; Esra Doger; Hamdi Cihan Emeksiz; Ozge Yüce; Aysun Bideci

Gazi University Medical Faculty, Pediatric Endocrinology, Ankara, Turkey

Aim and background: Total fat mass play a crucial role in the development of the metabolic complications. The aim of the study was to evaluate the relationship between metabolic syndrome risk factors and total body fat percentage.

Materials and methods: A total of 112 children and adolescents (84 obese subjects and 40 controls) aged between 10-16 years of age were enrolled in this study. Obese patients were also subgrouped according to the presence or absence of metabolic syndrome (MS) as "MS (+) obese " and " MS ( - ) obese " ( $n=45$ and $n=39$, respectively). Serum levels of glucose, insulin, HDL, LDL, cholesterol and trigliserit levels of all the participants were assessed after a12-hour fasting. Bioelectrical impedance apparatus was used to measure the percentage of body fat.

Results: Percentage of total body fat - standard deviation score (BF\%-SDS) was significantly correlated with body mass index (BMI), BMI-SDS, waist circumference, systolic and diastolic blood pressure, fasting insulin, HOMAIR, triglyceride and HDL. Whereas, there was no correlation between $\mathrm{BF} \%$ SDS and fasting blood glucose, total cholesterol and LDL. In the multiple regression analysis model, only systolic blood pressure and HOMA-IR retained their statistical significance upon $\% \mathrm{BF}-\mathrm{SDS}(\mathrm{t}=3.54, \mathrm{p}=0.001 ; \mathrm{t}=3.05$, $\mathrm{p}=0.003$, respectively).

Conclusions: A significant positive association was found between body fat percentage and metabolic risk factors.

\section{EPO-104 Fat Metabolism, Obesity \\ Clinicodiagnostic value of HbA1c in the prognosis of the metabolic syndrome in children and adolescents with exogenous constitutional obesity in Uzbek population} Shahnoza Azimova; Gulnara Rakhimova

Center for the Scientific and Clinical Study of Endocrinology, Children endocrinology, Tashkent, Uzbekistan

Background: To assess metabolic syndrome (MS) risk factors in children and adolescents with exogenous-constitutional obesity (ECO).

Materials and methods: We examined Uzbek 100 children and adolescents with ECO aged from 6 to 16. Modification of Bayes's method, that is, the one of norm-based intensive parameters [Shigan E.N., 1986] underlies the prognostic scale.

Results and discussion: Integral analysis demonstrated high MS risk in patients with ECO and lipid disorders. Thus, MS risk was 8.5 times higher ( 26.5 $\pm 3.1)$ in patients with triglycerides $(\mathrm{TG}) \geq 1.7 \mathrm{mmol} / 1 ; 5.5$ times higher $(44.8 \pm$ 8.5) with $\mathrm{HDL}<1.03 \mathrm{mmol} / \mathrm{l} ; 3.1$ times higher with $\mathrm{BMI}>97$ percentile $(45.2 \pm 14.7) ; 2.6$ times higher with $\mathrm{HbA} 1 \mathrm{c}>6.7 \%(40.0 \pm 15.4)$ and 2.2 times higher with atherogenicity index (AI) $>3.0(29.4 \pm 13.6)$. MS risk increases by 2.7 times $(50.7 \pm 18.8)$ upon early obesity onset (under 10 years of age), growing by 3.4 times $(42.9 \pm 12.5)$ upon ECO duration $>5$ years. Determination 
of relative risk (RR) and etiological fraction (EF) of MS risk factors demonstrated that $\mathrm{MS}$ is almost completely preconditioned with $\mathrm{TG} \geq 1.7 \mathrm{mmol} / \mathrm{l}$ $(\mathrm{RR}=8.47, \mathrm{EF}=88.19 \%)$ and $\mathrm{HDL}<1.03 \mathrm{mmol} / \mathrm{l}(\mathrm{RR}=5.30, \mathrm{EF}=81.15 \%)$. Very high and high MS precondition is associated with BMI $>97$ percentile $(\mathrm{RR}=3.07, \mathrm{EF}=67.44 \%), \mathrm{HbA} 1 \mathrm{c}>6.7 \%(\mathrm{RR}=2.60, \mathrm{EF}=61,54 \%)$ and $\mathrm{AI}>3.0$ $(\mathrm{RR}+2.16, \mathrm{EF}=53.64 \%)$

Conclusion: The integral estimation showed that children and adolescents under 16 years of age with high TG concentrations, low level of HLD, BMI $>97$ percentile, high glycated hemoglobin $>6.7 \%$ and disease duration more than 5 years are at the high risk of metabolic syndrome progression.

\section{EP0-105 Fat Metabolism, Obesity \\ Prevalence of high blood pressure in overweight and obese older children and adolescents}

Igor Lebid'; Nataliya Zelinska²; Vira Yakovenko' ; Tatiana Kobez'

${ }^{1}$ Crimean State Medical University, Periatric Department, Simferopol,

Ukraine; '2Ukrainian Center of Endocrine Surgery and Transplantation of Endocrine Organs and Tissues Ministry of Healths of Ukraine,

Department of Children Endocrinology, Kiev, Ukraine

Background: Childhood obesity $(\mathrm{O})$ has more than tripled in the past 30 years. Overweight (OW) and obese children are more likely to develop cardiovascular diseases at a younger age. Childhood $\mathrm{O}$ is associated with a higher chance of premature death and disability from cardiovascular disease in adulthood.

Objective and hypotheses: The aim of the study: to analyze prevalence of high blood pressure (HBP) among OW and O children. Methods: Target group: elder children and adolescents 10-18 y.o.: 30 OW children (10-14 y.o. - 10 children (33.33\%), 15-18 y.o. - $20(66.67 \%)), 56$ O children (10-14 y.o. 29 children $(51,78 \%), 15-18$ y.o. $-27(48,22 \%))$.

Diagnostic criteria: Body mass index (BMI) over the 85 -th percentile for age and sex (CDC, 2000) for OW children, BMI over 95-th percentile for age and sex for O children, HBP - blood pressure exceeding 95 percentile for age and sex. Family anamnesis of hypertension was investigated.

Results: About a half of OW and O elder children and adolescents $(42.31 \%$ of OW and $54.17 \%$ of $\mathrm{O}$ children) have family anamnesis of hypertension. The highest rate of $\mathrm{HBP}$ was within $\mathrm{O}$ group ( $1 / 2$ of $\mathrm{O}$ patients). Importantly $1 / 3$ of OW children have HBP. In both groups predominant increase in systolic BP, compared with diastolic BP, especially in children 10-14 y.o. But risk of diastolic BP increase rises with age. In older groups the risk for HBP doubles in $\mathrm{OW}$ and increases more than twice in $\mathrm{O}$ patients. Obese patients 15-18 y.o. have the highest risk of developing HBP, almost $2 / 3$ of them will have the indicated state. In OW patients there's no significant difference in the HBP prevalence according to gender, but in $\mathrm{O}$ patients only $1 / 3$ of girls will have HBP corresponding to $1 / 2$ of boys.

\begin{tabular}{lcccc} 
Children & $\begin{array}{c}\text { Overweight } \\
\text { Systolic BP }\end{array}$ & $\begin{array}{c}(\mathbf{n}=\mathbf{3 0}) \\
\text { Diastolic BP }\end{array}$ & $\begin{array}{c}\text { Obese } \\
\text { Systolic BP }\end{array}$ & $\begin{array}{c}(\mathbf{n}=65) \\
\text { Diastolic BP }\end{array}$ \\
\hline All & 30.00 & 13.33 & 48.21 & 20.36 \\
\hline $10-14$ y.o. & 20.00 & 0.00 & 27.59 & 7.00 \\
\hline $15-18$ y.o. & 35.00 & 20.00 & 70.37 & 55.56 \\
\hline Female & 31.25 & 12.50 & 33.33 & 26.67 \\
Male & 28.57 & 14.29 & 53.66 & 31.71
\end{tabular}

Conclusions: BP should be closely investigated for all patients with BMI over the 85-th percentile (in $\mathrm{O}$ and $\mathrm{OW}$ patients). Age, gender and positive family anamnesis are the main risk factors for development of HBP in future. Systolic BP increment is an early sign of HPB both in OW and O patients.

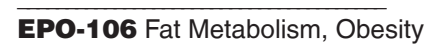

Metabolic syndrome prevalence has doubled in five years

Mehmet Emre Atabek; Beray Selver Eklioglu; Sevil Ari Yuca;

Nesibe Akyurek

Necmettin Erbakan University School of Medicine, Division of Pediatric

Endocrinology and Diabetes, Konya, Turkey

Background: Metabolic syndrome is one of the most important complications of obesity. Metabolic syndrome prevalence varies according to diagnostic criteria and populations.

Objective: We found the prevalence of metabolic syndrome as $27.2 \%$ in 2006. Our aim was to reveal a change in the prevalence of metabolic syndrome in the province of Konya, in five years.

Methods: We studied 202 obese children and adolescents (body mass index $>$ 95th percentile) aged between 7 and 18 years. The diagnosis of impaired glucose tolerance, type 2 diabetes and metabolic syndrome were defined according to modified WHO criteria adapted for children.

Results: Metabolic syndrome was found in $56,4 \%$, with a significantly higher rate among adolescents aged $12-18$ years $(63,2 \%)$ than among prepubertal children aged $7-11$ years $(47 \%)(\mathrm{p}=0.01)$. The prevalences of insulin resistance, glucose intolerance and type 2 diabetes were 60,8 and $2 \%$ among prepubertal children and $81,8,12.8$ and $0 \%$ among adolescents, respectively. The prevalence of fasting hyperinsulinemia in adolescents was significantly higher than prepubertal children $(\mathrm{p}<0.001)$. Hypertension was significantly more common in adolescents $(42,8 \%)$ than prepubertal children $(32,9 \%)(\mathrm{p}$ $=0.04$ ).

Conclusions: We found the incidence of metabolic syndrome in the city center of Konya approximate doubling of the last five years, and we found increased rates of morbidity with abnormal lipid profiles.

\section{EPO-107 Fat Metabolism, Obesity \\ A case of rapid-onset obesity with hypothalamic dysfunction, hypoventilation, autonomic dysregulation and neural crest tumor: Rohhadnet syndrome \\ Ayhan Abaci'; Gonul Catli'; Erhan Bayram; Tolga Koroglu; H. Nur Olgun ; Kamer Mutafoglü; A. Semra Hiz²; Ece Bober ${ }^{1}$ Dokuz Eylul University Faculty of Medicine, Pediatric Endocrinology Department, Izmir, Turkey; ${ }^{2}$ Dokuz Eylul University Faculty of Medicine, Pediatric Neurology Department, Izmir, Turkey; ${ }^{3}$ Dokuz Eylul University Faculty of Medicine, Pediatric Intensive Care Unit, Izmir, Turkey; ${ }^{4}$ Dokuz Eylul University Faculty of Medicine, Pediatric Oncology Department, Izmir, Turkey}

Background: Rapid-onset obesity with hypoventilation, hypothalamic dysfunction and autonomic dysregulation is a rare disorder which mimics common obesity and several endocrine disorders during early childhood.

Objective and hypotheses: To present the clinical features of a patient with ROHHADNET syndrome.

Methods: A 26 month-old boy was admitted to our emergency department with dispnea and cyanosis. He has gained weight of 8 kilograms in a period of two months. On physical examination his weight was $20 \mathrm{~kg}(+3.4 \mathrm{SDS})$, with a height of $97 \mathrm{~cm}(+3.2 \mathrm{SDS})$ and BMI $21.2 \mathrm{~kg} / \mathrm{m} 2(+2.8 \mathrm{SDS})$. He had central cyanosis with a slow and shallow respiration.

Results: Despite an absence of primary pulmonary, cardiac, neuromuscular disease or a causative brain stem lesion, he didn't have spontaneous breathing and a tracheostomy was performed.

Endocrine function tests revealed mild hyperprolactinemia $(44.7 \mathrm{ng} / \mathrm{mL})$ and failure of growth hormone response in glucagon stimulation test. Basal thyroid function tests showed a low TSH $(0.35 \mu \mathrm{IU} / \mathrm{mL}$, normal range $0,4-4)$ with a low free T4 $(0.75 \mathrm{ng} / \mathrm{dL}$, normal range $0.89-1.76)$ and normal free T3 (3.79 pg/dL, normal range 1.57- 4.71). No TSH response in a TRH stimulation test indicated secondary hypothyroidism.

Alveolar hypoventilation, rapid-onset obesity, hyperprolactinemia, central hypothyroidism, failed growth hormone stimulation test and absence of PHOX2B mutation on molecular analysis led to a diagnosis of ROHHAD syndrome.

Based on the association of ROHHAD syndrome with neural crest tumors, a screening thoracal and abdominal magnetic resonance imaging (MRI) was performed and a ganglioneuroblastoma, $6.5 \times 2 \times 3.5 \mathrm{~cm}$ in diameters, was detected. 
Conclusions: Because of the high prevalence of cardiorespiratory arrest and probability of accompanying tumors early recognition of ROHHAD syndrome is essential. To prevent presumptive mortality and morbidity ROHHAD syndrome should be considered in all cases of rapid and early onset obesity associated with hypothalamic- pituitary endocrine dysfunctions.

\section{EPO-108 Fat Metabolism, Obesity \\ Analysis of prenatal and perinatal factors associated with childhood obesity.}

\section{A case-control study}

Laura Losada'; Diego Yeste'; Silvia Grau²; Lina Velazquez²; Diana Bareño ${ }^{2}$; Antonio Carrascosa ${ }^{2}$

${ }^{1}$ Hospital Vall D'Hebron, Endocrinology, Barcelona, Spain; ${ }^{2}$ Hospital

Vall D’Hebron, Endocrinology Paediatric, Barcelona, Spain

Introduction: Obesity is the result of complex interactions among genetic, metabolic, perinatal and environmental factors.

Aims: To determine prenatal and perinatal factors associated with obesity in a population of obese children referred to our Paediatric Obesity Unit compared to healthy controls.

Patients and methods: Prospective cohort study including all children referred for obesity $(\mathrm{n}=173)$ in 2011 and a healthy control group (n:148).

Evaluated variables: Anthropometric data: weight, height, body mass index (BMI), waist circumference, blood pressure. Family history: parental weight and height and history of diabetes, hypertension and obesity in first- and second- degree relatives. Maternal history: age of mother at birth, gestational diabetes, pre-eclampsia, consumption of toxic substances (alcohol, tobacco) and duration of exclusive breastfeeding. Neonatal anthropometric data. Results: See Table

\begin{tabular}{|c|c|c|c|c|}
\hline & & Healthy $n=148$ & Obese $n=173$ & $p$ \\
\hline Age & Mean & $10.04 \pm 3.05$ & $11.61 \pm 3.2$ & 0.39 \\
\hline \multirow[t]{2}{*}{ Sex distribution } & Boys & $51.3 \%$ & $54.3 \%$ & 0.84 \\
\hline & Girls & $48.6 \%$ & $45.6 \%$ & \\
\hline BMI (SD) & Mean & $-0.01 \pm 0.88$ & $3.57 \pm 1.42$ & 0.00 \\
\hline Maternal BMI (kg/m2) & & $25.2 \pm 5.70$ & $29.71 \pm 6.02$ & 0.04 \\
\hline Paternal BMI (kg/m2) & & $26.5 \pm 3.43$ & $30.2 \pm 6.05$ & 0.001 \\
\hline Mother's age (years) & & $30.5 \pm 4.8$ & $30.3 \pm 5.20$ & 0.83 \\
\hline Birth Weight (g) & & $3187.1 \pm 587.14$ & $3263.6 \pm 677.15$ & 0.12 \\
\hline Birth length (cm) & & $49.6 \pm 2.10$ & $50.0 \pm 2.22$ & 0.50 \\
\hline Breastfeeding (months) & & $3.3 \pm 2.32$ & $2.5 \pm 2.55$ & 0.08 \\
\hline Familial diabetes mellitus & & $25.9 \%$ & $37.5 \%$ & 0.85 \\
\hline Familial obesity & & $38.7 \%$ & $81.8 \%$ & 0.00 \\
\hline Maternal obesity & & $15.6 \%$ & $26.4 \%$ & 0.03 \\
\hline Parental obesity & & $8.1 \%$ & $10.5 \%$ & 0.04 \\
\hline Maternal toxic consumption & & $27.5 \%$ & $38.9 \%$ & 0.08 \\
\hline Gestational diabetes & & $6.8 \%$ & $23.6 \%$ & 0.007 \\
\hline Maternal hypertension in pregnancy & & $4.0 \%$ & $9.8 \%$ & 0.85 \\
\hline
\end{tabular}

Conclusions: High parental BMI and a history of gestational diabetes are factors that could contribute to childhood obesity. This population should be subject to special control of weight gain in infancy.

\section{EP0-109 Fat Metabolism, Obesity}

\section{5-Hydroxyvitamin D and metabolic risk in children}

Hye Ah Lee'; Young Ju Kim²; Hwayoung Lee ; Hye Sun Gwak'; Eun Ae Park; ; Sujin Cho ; Se Young Oh ${ }^{6}$; Eun Hee Ha ${ }^{1}$; Hyesook Park ${ }^{1}$ Ewha Womans University, Department of Preventive Medicine, Seoul, Republic of Korea; ' 2 Ewha Womans University Mokdong Hospital, Obstetiric Departement, Seoul, Republic of Korea; ${ }^{3}$ Ewha Womans University Mokdong Hospital, Anatomy, Seoul, Republic of Korea; ${ }^{4}$ Ewha Womans University, Colleage of Pharmacy, Seoul, Republic of Korea; ${ }^{5}$ Ewha Womans University Mokdong Hospital, Pediatric Departement, Seoul, Republic of Korea; ${ }^{6}$ Kyung Hee University, Department of Food and Nutritionement, Seoul, Republic of Korea; ${ }^{7}$ Ewha Womans University Mokdong Hospital, Department of Preventive Medicin, Seoul, Republic of Korea

Objective and hypotheses: The lower 25-Hydroxyvitamin $\mathrm{D}(25(\mathrm{OH})$ D) is being suggested as a risk factor for Type 2 diabetes, but there is not well construed for its relationship. Therefore, we aimed to assess the association among 25(OH)D level, metabolic syndrome components [Waist circumference(WC), Blood pressure, Triglycerides, Glucose, High-Density lipoprotein Cholesterol], and insulin resistance indices [homeostasis model assessment-insulin resistance, quantitative insulin-sensitivity resistance, glucose to insulin ratio among preadolescent child.

Methods: We followed up 221 subjects from July to August 2011 aged 7 to 9 child, who were part of Ewha Birth \& Growth Cohort study, Seoul, Korea, which is a prospective cohort established 2001-2006. We investigated the associations among vitamin D level in blood, metabolic syndrome components, and insulin resistance using multivariate regression analysis adjusted for sex, age, birth weight, calories, and BMI z score.

Results: $38(17.2 \%)$ child were deficiency $(<20 \mathrm{ng} / \mathrm{mL})$ for vitamin D level, and its prevalence was more higher in boys $(25.2 \%)$ than girls $(8.1 \%)$. There was significant relationship between $25(\mathrm{OH}) \mathrm{D}$ and triglycerides $(\mathrm{B}=-0.01$, $\mathrm{p}=0.04$ ) adjusted for sex, age, birth weight, calories, and BMI z score. WC was also negatively associated with $25(\mathrm{OH}) \mathrm{D}(\mathrm{B}=-0.13$, $\mathrm{p}=0.07)$ with cofactors, although its relationship was shown the marginal boundary. But, other features were not associated with $25(\mathrm{OH}) \mathrm{D}$. When regarding the criteria for metabolic components, those who were more than WC $90 \%$ tile had higher frequency in deficient group than in sufficient group, but distribution of metabolic components among 25(OH)D status was no significant difference. Conclusions: The lower 25(OH)D level may contribute to the association with some of metabolic components in general preadolescent child, but further study is needed to explore the relationship with insulin resistance.

\section{EPO-110 Fat Metabolism, Obesity}

\section{Triglyceride/HDL-cholesterol ratio as a marker of insulin resistance in overweight and obese children}

Kyriaki Tsiroukidou'; 'Konstantinos Kitsios'; Elpida Hatziagorou'; Konstantina Kosta'; Maria Papagianni'; Anastasios Vamvakis'; Maria Papadopoulou ${ }^{1}$

${ }^{1}$ Aristotle University of Thessaloniki, 3rd Paediatric Department, Thessaloniki, Greece; ${ }^{2}$ Gennimatas General Hospital of Thessaloniki, Internal Medicine, Thessaloniki, Greece

Background: Obesity dyring childhood is often related to type 2 Diabetes, dyslipidemia and metabolic syndrome. HOMA-IR index is widely used to calculate insulin resistance and Triglycerides/HDL ratio to determine dyslipidemia.

Objective and hypotheses: To determine HOMA-IR levels and Triglycerides/High Density Cholesterol (Tgl/HDL) ratio in overweight and obese children compared to normal weight counterparts. To associate $\mathrm{Tgl} / \mathrm{HDL}$ ratio with the risk for insulin resistance.

Methods: The study included 190 children [(87 males (45.8\%)] aged 8-16 years old (mean age $11 \pm 2.3$ years). $\mathrm{Tgl} / \mathrm{HDL}$ ratio was calculated in 66 overweight (body weight $60.55 \pm 10.1 \mathrm{~kg}, \mathrm{BMI} 25.9 \pm 1.6 \mathrm{~kg} / \mathrm{m} 2$ ), 84 obese (body weight $69.6 \pm 19.7 \mathrm{~kg}$, BMI $30.2 \pm 4.5 \mathrm{~kg} / \mathrm{m} 2$ ) and 40 normal weight (body weight $32.9 \pm 13.2 \mathrm{~kg}$, BMI $17.45 \pm 2.9 \mathrm{~kg} / \mathrm{m} 2$ ) children. Correlation between Homa-IR index and $\mathrm{Tgl} / \mathrm{HDL}$ ratio was studied in all groups.

Results: Tgl/HDL ratio was found to be significantly raised in obese children $(2.57 \pm 2.3, \mathrm{p}<0.0001)$ compared to normal weight counterparts $(1.09 \pm 0.6$ $\mathrm{p}<0.0001)$ as well as in overweight participants $(1.87 \pm 1.17)$. Mean $\mathrm{Tgl} /$ 
HDL ratio was also significantly higher in the obese group compared to the overweight one $(\mathrm{p}=0.019)$. Homa-IR index was found increased in obese $(5.13 \pm 4.19, \mathrm{p}<0.0001)$ and overweight $(3.47 \pm 2.44, \mathrm{p}<0.0001)$ groups compared to normal weight individuals $(1.81 \pm 0.96)$ but also compared to each one of the groups separately $(\mathrm{p}=0.003)$. Strong relation between Homa-IR index and $\mathrm{Tgl} / \mathrm{HDL}$ ratio was found $(\mathrm{r}=0.275, \mathrm{p}<0.0001)$.

Conclusions: Homa-IR index and $\mathrm{Tgl} / \mathrm{HDL}$ ratio increase significantly in overweight and obese children. We suggest that $\mathrm{Tgl} / \mathrm{HDL}$ ratio can be used as a simple and useful marker to identify children at risk for insulin resistance.

\section{EPO-111 Fat Metabolism, Obesity \\ Clinical Characteristics of the children with overweight or obesity \\ Eun Hui Hong \\ Gumi CHA University Hospital, Pediatrics, Gumi, Republic of Korea}

Purpose: The aim of this study was to evaluate clinical characteristics on children with overweight or obesity.

Methods: The subject for this research consisted of 86 patients ( 25 boys and 61 girls) who were admitted to the department of Pediatrics at Gumi CHA University Hospital from August of 2010 to February of 2012. They admitted to investigate their growing status with concerning overweight. Various clinical or laboratory data were analyzed in the way of retrospective study.

Results: Among them, 11 patients were diagnosed as central precocious puberty, 3 patients were diagnosed as Hashimoto's thyroiditis and 1 patient was diagnosed as growth hormone defiency. 56 patients $(65 \%)$ shows insulin resistance and 14 patients(16.3\%) shows acanthosis nigrans.

Conclusions: We should consider that obesity isn't just symptom but a disease. Clinicians need to investigate their comorbidity and to take appropriate treatment.

\section{EPO-112 Fat Metabolism, Obesity}

\section{The relative influence of social reactions and health concerns on weight management motivation of adolescents}

Sabine Herget'; Fabian Kruse'; ${ }^{2}$ Lenneke van Eerd²; Anton Dijker²; Sonia Lippke ${ }^{3}$

${ }^{1}$ Leipzig University, IFB AdiposityDiseases, Leipzig, Germany; ${ }^{2}$ Maastricht University, Department of Health Promotion, Maastricht, Netherlands; ${ }^{3}$ Jacobs University Bremen, Jacobs Center on Lifelong Learning and Institutional Development, Bremen, Germany

Background: As obesity has become a global public health problem, associated weight discrimination is often investigated. However, there has been little focus beyond stigmatization on a broader range of social reactions and their impact on weight control motivation.

Objective and hypothesis: The relative influence of social reactions and health risks on motivation and intention for weight control among adolescents were examined. The following hypotheses were explored:

1) Both health risks of obesity and social reactions would serve as a considerable motivation for weight control.

2) Positive and supporting statements would lead to greater weight control motivation than negative commentaries.

3) The responsiveness to social reactions, represented by increased motivation for weight control, plays an important role in foretelling behavioral intention for overall future weight management.

Population and methods: A quantitative survey study was carried out among 546 students (age: $19.88 \pm 2.8$ ) of vocational training schools. Participants were subjected to a questionnaire on health risks and to social reactions on weight. The respondents' motivation and overall behavioral intention for weight management was measured.

Results: Participants were strongly motivated by the risk of health problems, common situational experiences of overweight and by support and concern, severely negative commentaries and advice to lose weight. Behavioral intention for future weight management was predicted by responsiveness to support, negative commentaries, advice, frustration of others, situational experiences and stigma by association, but not by expected health problems.

Conclusion: Various social reactions exert- besides knowledge on health risks- considerable influence on weight control motivation and weight management intention of young adults and may therefore need to be taken into account for future design of effective weight control interventions.

\section{EP0-113 Fat Metabolism, Obesity}

After care treatment approach for adolescent obesity via telephone counseling following an obesity treatment program - study concept Jana Markert' ; Sabine Herget ${ }^{1}$; Mandy Vogef; Ruth Gausche ${ }^{2}$; Anja Hilbert' ${ }^{1}$; Susann Blüher ${ }^{1}$

'IFB Adiposity Diseases, Faculty of Medicine, University of Leipzig, Leipzig, Germany; ${ }^{2} \mathrm{CrescNet}$ gGmbH, University of Leipzig, Leipzig, Germany

Background: The purpose of obesity treatment is to sustainably reduce body weight and to achieve lifestyle changes, but maintaining weight loss after obesity therapy remains a challenge.

Objective and hypotheses: This study aims to evaluate feasibility and efficacy of a structured aftercare program for adolescents aged 12.5-17.5 years after obesity therapy. The core of the aftercare intervention will be telephone counseling. The following questions shall be explored: Which percentage of addressed candidates can be included in the program? Which percentage of recruited adolescents attends to the program? Are there predictors for program acceptance/program attendance? What is the programs' impact on maintaining BMI-SDS, and on implementing a healthy lifestyle?

Subjects: Inclusion criteria: 12.5-17.5 years, completion of a structured obesity therapy program. Exclusion criteria: current involvement in weight loss treatment, psychiatric conditions interfering with participation.

Methods: Recruitment shall be performed via obesity in- and outpatient therapies. Data from all addressed candidates will be documented. Candidates willing to participate will be randomized into an intervention (telephone counseling) and a control (information material only) group. The intervention starts within a period of six weeks after completion of obesity therapy. It consists of seven telephone counseling interviews (15-20 min) over a period of six months: one enrollment interview, three basic interviews, two coaching interviews, one final interview. Adolescents will be addressed directly. To ensure sustainability within the family setting the first coaching interview will be held with the adolescent and his/her parents together. In order to foster the exchange between the participants a web-blog will be installed. One and two years following randomization follow-up assessments will be conducted. Discussion: We hypothezise that a 6-months aftercare treatment approach for adolescents based on telephone counselling will have a sustainable effect on maintaining body weight.

\section{EPO-114 Fat Metabolism, Obesity \\ Incidence of dyslipidaemia is higher in children with risk factors of CVD than other children} Chong Kun Cheon; Yong Suk Kim; Tae Min Eeom; Su Young Kim Pusan National University Childrens' Hospital, Pediatrics, Yangsan, Gyeongnam, Republic of Korea

Background: In adults, several large prospective population-based studies have shown that higher risk of CVD is associated with multiple risk factors (eg, increased body-mass index, hypertension, dyslipidemia, and family history of CVD). While data linking these risk factors to cardiovascular events are limited in children, children with specific disease states (eg, familial hypercholesterolemia, diabetes mellitus, and renal failure) are more likely to develop premature atherosclerosis and CVD at an earlier age. In Korea there has been no data in prevalence of dyslipidemia in children with or without risk factors in children.

Objective and hypotheses: We tried to get the incidence of dyslipidemia in risky conditions for CVD and other condition in children.

Methods: We reviewed all of the labaratory data concerning lipid metabolism in patients who visited or admitted to our hospital during recent 3 years. We compared the incidence of dyslipidemia between high risk group for CVD and other children.Dyslipidemia was defined one or more of following criteria. Total cholesterol $>200 \mathrm{mg} / \mathrm{dl}$, HDL-C $<40 \mathrm{mg} / \mathrm{dl}$, LDL-C $>130 \mathrm{mg} / \mathrm{dl}$, TG $>150 \mathrm{mg} / \mathrm{dl}$ in adolescents, or $>130 \mathrm{mg} / \mathrm{dl}$ in children. Risk group was definded in patients with overweight/obesity, hypertension, familial hypercholesterolemia, CKD, Kawasaki disease, cancer, CHD like COA or TGA, chronic inflammatory disease like SLE, JRA, inflammatory bowel disease, diabetes mellitus.

Results: Incidences of dyslipidemia of children with risk factors were $24.2 \%$ in total cholesterol, $22.8 \%$ in HDL-C, $23.8 \%$ in LDL-C, $44.3 \%$ in TG, compared to $10.8 \%$ (T. chol), $10.8 \%$ (HDL-C), 9.8\% (LDL-C), 28.8\% (TG) in other children. 
Conclusions: The incidence of dysplipidemia in all categories of lipid was higher in risk group So intense management for dyslipidemia and disease itself are needed in children with risk factors for CVD

\section{EPO-115 Fat Metabolism, Obesity}

\section{Alanine transaminase as a marker of non- alcoholic fatty liver disease in a group of obese children}

Corina Pienar; Monica Marazan; Adela Chirita Emandi; Ioana Micle "Louis Turcanu" Emergency Hospital for Chidren, 1st Pediatric Clinic, Timisoara, Romania

Background: Nonalcoholic Fatty Liver Disease (NAFLD) has become an important cause of liver disease in pediatric populations worldwide. It is believed that fat accumulation within the liver is a major step in the development of insulin resistance and the metabolic syndrome. Alanine transaminase (ALT) is frequently used as a surrogate marker to estimate NAFLD

Objective and hypotheses: We investigated the relationship between ALT levels, insulin resistance and the presence of the metabolic syndrome in a group of obese Romanian children.

Methods: We studied 40 obese children evaluated in our department between January 1st 2008 and December 31st 2009. Analyzed data included all the metabolic syndrome criteria as defined by Weiss et al., ALT, baseline insulin and the homeostatic model assessment: insulin resistance (HOMA-IR). We examined the ALT profile of the study group across insulin resistance and metabolic syndrome categories, respectively. We used the Mann-Whitney test to verify the significance of our findings.

Results: We found significant higher ALT values across insulin resistance categories $(p=0.014)$. We did not find significant differences between ALT and HOMA-IR values across metabolic syndrome categories $(p=0.240, p=$ 0.122 ).

Conclusions: Obese insulin resistant children were also identified with more elevated ALT levels. Obese children showed similar ALT and insulin resistance profiles, independent of their metabolic syndrome status. Further diagnosis studies, including liver ultrasound, and close follow-up are needed.

\section{EPO-116 Fat Metabolism, Obesity}

\section{Early detection of hyperinsulinism and risk factors for metabolic syndrome in prepubertal children}

Francesco Gallo ${ }^{1}$; Pietro Conte ${ }^{1}$; Giuditta De Quarto ${ }^{1}$; Albina Tummolo Vito Antonio Caiulo'; Fulvio Moramarco'

${ }^{1}$ ASL BR Perrino Hospital, U.O. Pediatrics, Brindisi, Italy; ${ }^{2}$ Giovanni

XXIII Hospital, U.O. Genetica Clinica, Bari, Italy

Background: The definition of metabolic syndrome in pediatric age is controversial and seems to be incorrect to make this diagnosis in children under $10 \mathrm{y}$. Hence, HOMA-IR is the most common way to define insulin resistance, even if sensibility and sensitivity are not ideal. Rarely, hyperinsulinism was detected with standard oral glucose tolerance test (OGTT), especially in prepubertal children.

Objective and hypotheses: Early detection of cardiovascular risk factors in Tanner stage 1 children, to prepare a long term study about the influence of precocious exposure to this factors on obesity complications development.

Methods: From January 2009 to December 2011 we observed 155 obese children in day hospital: M 74, F 81; age $6.82+/-1.54$, Tanner stage 1, BMI-SDS $>2$, waist circumference $>2 \mathrm{SDS}, \mathrm{W} / \mathrm{H}>0.5$, normal blood pressure). We measured fasting glycaemia (normal values $<95 \mathrm{mg} / \mathrm{dl}$ ) and insulin $(<15$ $\mathrm{mU} / \mathrm{L}), \operatorname{HDL}$ (n.v. $>40 \mathrm{mg} / \mathrm{dl}$ ), triglyceridemia (n.v. $<100 \mathrm{mg} / \mathrm{dl}$ ). In 29 of them ( $18.7 \%$ ) (including criteria: fasting glycaemia $>95$, fasting insulin $>15$ and/or acanthosis nigricans and/or familiarity for T2DM or precocious cardiovascular diseases without hyperdislipidemia) was evaluated $180^{\circ}$ OGTT $(1.75 \mathrm{~g} / \mathrm{kg})$ to measure glycaemia and insulinemia.

Results: Fasting insulinemia: median $18.91+/-1.62$, peak $107.23+/-73.74$ HDL median $51.4+/-12.3$, trygliceridemia $71+/-31.2$. Six children $(3.8 \%)$ responded to criteria for metabolic syndrome (hypoHDL + hypertriglyceridemia + visceral obesity) but none of them had hyperinsulinism.

Hyperinsulinism was found in 15 patients $(51.7 \%)$; 9 (60.7 \% of hyperinsulinemic children), had hypoHDL or hypertriglyceridemia.
Conclusions: We founded precocious spies of future CV diseases in very young children. Our opinion is that it's necessary to identify as soon as possible the high risk children in order to modify their lifestyle and prevent the tremendous "tide" of obesity, T2DM and CV diseases

\section{EPO-117 Fat Metabolism, Obesity \\ Alterations of carbohydrate metabolism in obese children: incidence and severity}

Corina Paul; Ionela Tamasan; Iulian Velea

University of Medicine and Pharmacy, Clinic II Pediatrics, Timisoara, Romania

Background: It is wellknown that obesity is associated with disturbances of the carbohydrates metabolism. These include impaired fasting glucose (IFG) and impaired glucose tolerance (IGT) (known as prediabetes), hypoglycemias and even type II diabetes mellitus (T2DM).

Objective and hypotheses: To evaluate the incidence and severity of carbohydrates metabolism disturbances in a group of obese children followed-up in Clinic II Pediatrics.

Methods: Within 2007-2011, we studied 105 children with primary obesity. Their clinical evaluation included anthropometric indexes (weight, height, BMI) and clinical elements relevant for insulin resistance (purple striae, acanthosis nigricans); biologically we determined fasting blood glucose and within OGTT, lipid profile (cholesterol, HDL cholesterol, triglycerides), inflammation (CRP) and in selected cases insulinemia (basal or stimulated OGTT), C peptide, HbA1c, morning cortisol.

Results: At first visit 41 cases $(39 \%$ ) had mild obesity (weight excess $>20$ $30 \%), 40$ cases $(38 \%)$ medium obesity (w.e. $30-50 \%)$ while 24 cases $(23 \%)$ were severely obese (w e. $>50 \%$ ). IFG was found in 4 cases with mild obesity, 4 cases with medium obesity and 2 cases with severe obesity. IGT was present in 6 cases with mild obesity, 6 cases with medium obesity and 4 cases with severe obesity. Fasting hypoglycemias were found in 2 mild, 2 medium and 4 severe obese children. 4 cases were diagnosed as T2DM; 3 associated with mild and 1 with severe obesity. We found hyperinsulinemia in 2 cases with T2DM (1 associated with severe obesity and 1 with mild obesity).

Conclusions: Obesity is associated with alterations of the carbohydrates metabolism. Precocious intervention concerning the nutritional habits of the child and family and their lifestyle would be helpful in order to avoid the occurrence of prediabetes or even T2DM.

\section{EPO-118 Fat Metabolism, Obesity \\ Overweight and obesity prevalence in 10-18 year children in Lithuania}

Natalija Smetanina'; Rasa Verkauskiene ${ }^{1}$; Apolinaras Zaborskis²; Giedre Mockeviciene ${ }^{1}$; Edita Jasinskiene ${ }^{1}$; Rimante Dobrovolskiene ${ }^{1}$; Indre Petraitiene ${ }^{1}$; Rima Berontiene'; Veslava Babinska ${ }^{3}$; Lina Zilyte ${ }^{3}$ 'Lithuanian University of Health Sciences, Medical Academy, Department of Pediatric Endocrinology, Kaunas, Lithuania; 'Lithuanian University of Health Sciences, Medical Academy, Faculty of Public Health, Kaunas, Lithuania; ${ }^{3}$ Lithuanian University of Health Sciences, Medical Academy, Kaunas, Lithuania

Background: The rising prevalence of overweight and obesity in children may have adverse long-term metabolic consequences for risks of type 2 diabetes, metabolic syndrome and cardiovascular diseases.

Objective and hypotheses: The aim of our study is to establish the prevalence of the overweight and obesity among a representative cohort of 10-18 year old children in Kaunas region (central part of Lithuania).

Methods: A school-based survey of children aged 10-18. Body height and weight were measured and body mass index (BMI) was calculated. Obesity and overweight were estimated using the International Obesity Task Force (IOTF) cut-off points.

Results: The study sample included 2194 children (1041 boys and 1153 girls) in the period of 2008-2009. Total prevalence of childhood overweight was $12.44 \%$ ( $12.77 \%$ for boys and $12.14 \%$ for girls respectively) and obesity $3.46 \%$ (4.03\% for boys and $2.94 \%$ for girls respectively).

Conclusions: In comparison to 2002 data in 2002 when overweight rate among $7-18$ aged children was $10.5 \%$ in boys and $9.9 \%$ in girls; obesity prevalence $2.9 \%$ in boys and $4.37 \%$ in girls, respectively, we found a slightly increasing tendency in 10-18 year adolescent overweight and obesity. 


\section{EP0-119 Fat Metabolism, Obesity}

Relevance and pitfalls of blood pressure measurements in clinical practice - differences between lean and obese children and

\section{adolescents}

Isabel Wagner; Kathrin Dittrich; Madlen Neef; Rita Ketzscher;

Wieland Kiess; Antje Körner

University of Leipzig, Dept. of Women's and Child Health, Leipzig,

Germany

Background: The incidence of hypertension in obese children and adolescents is increasing. Often, single blood pressure measurements (BPMs) are not sufficient to diagnose hypertension. The ideal diagnostic method and gold standard is a 24 h-ambulatory blood pressure measurement (ABPM). Especially in obese children, there are many difficulties and pitfalls with BPMs. Therefore, it was our aim to investigate variations between the two methods and to compare BP levels between lean and obese children and adolescents. Methods: We evaluated BPMs in 175 subjects (106 obese, 69 lean) aged 6.418.8 years. Random BPMs were performed on both arms with two immediate control measurements and additional control measurements after 30, 60 and 120 minutes.

In addition, a 24 h-ABPM with intervals ranging between 15 minutes during the day and 30 during the night was performed in each child.

Results: The incidence of hypertensive BP values was $21.5 \%$ when based on one single measurement, while it decreased to $12.9 \%$ and $8.6 \%$ in control measurements after 30 and 120 min respectively. In the 24 h-ABPM, 12.9\% of the children were diagnosed as being hypertensive ( $>95$. percentile).

We found significant differences in the systolic BP between lean (-0.10 SDS $-0.57 \mathrm{SDS})$ and obese (+0.63 SDS/ +0.36 SDS) children in random BPMs +0.73 SDS $(p<0.0001)$ and the ABPM +0.93 SDS $(p<0.0001)$. The systolic load was much higher in the obese $(21.2 \%)$ compared to the lean $(10.1 \%)$ group, while the difference of the diastolic load was $3.1 \%$.

Conclusion: Significant differences in BP levels between lean and obese children were present with a much higher incidence of hypertension in the obese group.

The high error rate in random BPMs underlines the need for control measurements, particularly if BP values are elevated. A 24 h-ABPM is still recommended if there is any suspicion of hypertension in lean or obese children and adolescents.

\section{EPO-120 Fat Metabolism, Obesity \\ Waist-to-height ratio an easier index for evaluating obesity in children and adolescents Meriem Bensalah"; Samia OuldKablia²; Zoubir SellaR; \\ Mustapha Chahi ${ }^{3}$; Zahra Kemali \\ ${ }^{1}$ Central Hospital of Army, Department of Endocrinology, Algiers, \\ Algeria; ${ }^{2}$ Central Hospital of Army, Endocrinology Department, Algiers, \\ Algeria; ${ }^{3}$ Salim Zemirli Hospital, Internal Medecine Department, \\ Algiers, Algeria}

Background: The normal body mass index (BMI) range, as defined by the World Health Organization (WHO), is quite wide, and some people within this range may have excessive central fat accumulation and elevated metabolic risks. The waist-to-height ratio $(\mathrm{W} / \mathrm{Ht})$ is an effective index for assessing central fat distribution among children and adolescents and give an excellent reflection of vascular risk.

Objective and hypotheses: The aim of this study is to determine prevalence of obesity and overweight in a population of children and adolescent and evaluate the values of waist /height ratio in normal, overweight and obese children and adolescents.

Methods: A cross-sectional study was conducted in the east of Algiers on a representative sample of 648 adolescents aged between 11 and 15 years. We have measured weight, height and waist circumference. Overweight and obesity were defined by Cole references.

Results: The prevalence of obesity and overweight was $24,9 \%$ (CI 95\%:21,627,3 ). Girls represented $48 \%$ of the studied population and $46,5 \%$ of the obese or overweight population. Meddle age was 14,5 years. There was a good correlation between BMI and waist circumference.

The report W/Ht was found high in $25 \%$ of the population, corresponding to the proportion of overweight or obese adolescents and was respectively: 0,440,52-0,57 in normal weight, overweight and obese children and adolescents (p:0,01).

51st Annual Meeting of the ESPE
Conclusions: Waist to height ratio is a good indicator of central fat accumulation in children and adolescents and may be better than BMI. Clinician must calculate this ratio to evaluate cardiovascular risk. A ratio greater than 0,5 predict central obesity.

\section{EPO-121 Fat Metabolism, Obesity \\ Prevalence of metabolic syndrome, frequency of cardiovascular risk factors and association of leptin level with metabolic syndrome and cardiovascular risk factors in Turkish obese children and adolescents \\ Boyraz Mehmet ${ }^{1}$; Peyami Cinaz ${ }^{2}$; Baki Karoglü; Emre Tapcilar ${ }^{3}$; \\ Aysun Bidecí; Orhun Çamurdan²; Hamdi Emeksiz ${ }^{2}$ \\ ${ }_{1}^{1}$ Pipli Etfal Training and Research Hospital, Pediatric Endocrinology, \\ Istanbul, Turkey; ${ }^{2}$ Gazi University Medical Faculty, Pediatric Endocrinology, Ankara, Turkey; ${ }^{3}$ GATA, Pediatric Endocrinology, \\ Ankara, Turkey}

Objective and hypotheses: Metabolic syndrome is a hyperleptinemic condition and serum leptin levels are positively associated with fat tissue mass, insulin resistance and type 2 diabetes. In this study, we aimed to determine the prevalence of metabolic syndrome, the frequency of cardiovascular risk factors in children with obesity and metabolic syndrome, and to evaluate the relation between metabolic syndrome and plasma leptin levels.

Methods: In this study, 451 adolescents and children aged between 8 - 18 years admitted with complaints of excess weight, body mass index above 95 th percentile for age and sex were enrolled as study group and 139 children served as control group. Insulin resistance or impaired glucose tolerance regarding WHO criterias, the diagnosis of MS was made when they additionally had dyslipidemia (hypertriglyceridemia or low HDL cholesterol level) and/ or hypertension

Results: In the study group 55\% were male and $45 \%$ were female. Mean age for girls was $12.2 \pm 2.2$ and $12.5 \pm 1.9$ year for boys. There were $96(21.2 \%)$ children with metabolic syndrome. It was found that leptin had a positive relationship with waist circumference, insulin level, HOMA-IR and LDL cholesterol level and a negative one with age, HDL cholesterol and triglyceride levels. Metabolic syndrome prevalence was high in obese adolescent children. Interestingly, leptin levels in obese and non-obese male and female patients were found to be different as well. After elimination of BMI and gender factors, this study showed that there was no significant difference in leptin levels. Even there was a strong relationship between obesity indices and leptin levels, this relation was not observed in cases with metabolic syndrome.

Conclusions: Further studies are required for evaluation of leptin levels in metabolic syndrome cases from different ethnicity.

\section{EPO-122 Fat Metabolism, Obesity}

\section{Relationship of adipokines with metabolic syndrome components in pubertal obese children}

Mehmet Boyraz'; Baki Karaoglu²; Aysun Bideci; Peyami Cinaz; Nihal Hatipoglu ${ }^{4}$

1 pipli Etfal Training and Research Hospital, Pediatric Endocrinology, Istanbul, Turkey; ${ }^{2}$ GATA, Pediatri, Ankara, Turkey; ${ }^{3}$ Gazi University Medical Faculty, Pediatric Endocrinology, Ankara, Turkey; ${ }^{4}$ Erciyes University Medical Faculty, Pediatric Endocrinology, Kayseri, Turkey

Objective and hypotheses: This study aimed (a) to investigate the relationship between metabolic syndrome (MS) and serum adiponectin, resistin, RBP-4; (b) to clarify the relationship between MS components and adipokines levels.

Methods: We studied 148 obese children and adolescents ( 82 boys and 66 girls; mean age was $12.3 \pm 2.4$ years). We defined obesity as a body-mass index (BMI) z-score more than 2 SD above the mean for age and sex. Fasting glucose, insulin levels and lipid profiles were measured in all cases and controls after a 12-hour fast. Adiponectin, resistin and RBP-4 levels were measured in the subjects.Obese patients were grouped into two subgroups according to whether they were grouped as MS or not. MS was defined based on the modified WHO criteria adapted for children.

Results: Baseline demographics are presented for the entire study cohort and 
the subsets of girl, boy, presence of MS, and non MS. BMI, WC, HOMAIR, blood pressure, serum levels of fasting glukoz, triglyceride, insulin and RBP4 were higher, whereas HDL, resistin and adiponectin levels were lower in the MS group when compared to the non MS group. In univariate analyses, adiponektin and resistin concentrations were significantly negative correlated with HOMA-IR, waist circumference, triglyceride, blood pressure and positive correlated HDL cholesterol. RBP-4 concentrations were significantly positive correlated with HOMA-IR, waist circumference, triglyceride, diastolic and systolic pressure and negative corelated HDL cholesterol. It was also analysed the results of adipokines in the groups of MS and non-MS Plasma adiponektin, resistin were faller and RBP-4 was higher in subjects with the metabolic syndrome compared with subjects without the metabolic syndrome. Adiponektin, and resistin concentrations were higher in women compared with men. However, RBP4 concentrations differed significantly between women and men.

Conclusions: Evaluation of serum adiponectin, resistin and RBP-4 levels might contribute to an early intervention in obese children with MS.

\section{EPO-123 Fat Metabolism, Obesity}

\section{Endothelial function in children and young and its relation to cardiovascular risk factors} María Cristina Bazán de Casella'; Alejandro Negrete²;

Claudio Joo Turoniz; Nicolás Salas²; Rodrigo Marañón'; Juan Salinas²; Maria Elisa Bruno²; Sofía Karbiner2; Maria Peral de Bruno ${ }^{2}$

${ }^{1}$ School Medicine Tucuman University, Endocrinology Pediatric, Tucumán, Argentina; ${ }^{2}$ Hospital del Niño Jesús Tucumán, Biomédico, Tucumán, Argentina

Background: Endothelial function (EF) is altered in obesity, hypertension, diabetes and metabolic syndrome (MetS), however, few studies were performed in children and young.

Objective: To determine EF in a young population and its relation to cardiovascular risk factors From outpatient Department of Endocrinology at the Hospital del Niño Jesus Tucumán (July to December 2011), we included children and youth are male and without diabetes, hypertension, history of previous illness, or risk of low weight (BMI percentile $<10 \%$ ). They are divided into obese $(\mathrm{n}=25)$ and nonobese $(\mathrm{n}=10)$. Anthropometric parameters, percentage of body fat, blood pressure and laboratory data were measured. EF and arterial stiffness index were measure by reactive hyperemia plethysmograph method noninvasive by pulse wave analysis.

Results: Had higher body fat percentage and waist circumference. BMI was correlated with the percentage of fat (R2: $0.3415, \mathrm{n}=23)$. Obese had significantly higher glucose values. Insulin and HOMA index were altered . EF was higher in non-obese $1.9 \pm 2.3 \%$ over baseline, $\mathrm{n}=9)$ than in obese $(14.4 \pm$ $0.5 \%, \mathrm{n}=23, \mathrm{P}<0.05$ ). Similarly, arterial stiffness index was higher in obeses. In obese this response was negatively correlated with age (R2: $0.2243, \mathrm{n}=23$, $\mathrm{P}<0.05$ ), waist circumference and HOMA index. While both groups had less than $97 \%$ percentiles of blood pressure, obesity presented a positive correlation between BMI and systolic blood pressure.

Conclusions: If well, obeses do not have the diagnostic criteria for MetS, they have insulin resistance, anthropometric measures altered and fat gain. The correlation between BMI and blood pressure in these stages, without hypertension, it would mark a tendency to be studied. Obese people have lower $\mathrm{FE}$, indicating early vascular changes and that as they grow more this FE deteriorate. The negative correlation between FE and HOMA index and waist circumference indicating that even without MS, this population already has changes indicating the importance of studying this population, even before this SM, preventing, and the occurrence of cardiovascular disease.

\section{EPO-124 GH and IGF Physiology}

Abstract withdrawn.

\section{EPO-125 GH and IGF Physiology}

GH deficiency in Rubinstein-Taybi syndrome: a possible link between molecular basis (CREBBP mutations) and the short stature in

\section{these patients}

Pierluigi Marzuillo; Anna Grandone; Raffaella Nacca; Adalgisa Festa; Ruggero Coppola; Federica Messa; Emanuele Miraglia del Giudice; Laura Perrone

SUN, Pediatrics, Napoli, Italy

Background: Rubinstein-Taybi syndrome (RTS) is a rare autosomal dominant disorder (prevalence 1:125000) characterised by broad thumbs and halluces, facial dysmorphisms, psychomotor development delay, skeletal defects, abnormalities in the posterior fossa and short stature. The known genetic causes are point mutations or deletions of the cAMP-response element binding protein (CREBBP) (50-60\% of the cases) and p300 (5\%), homologus gene.

Objective and hypotheses: We suppose that the short stature in RubinsteinTaybi Syndrome with CREBBP mutation could be determinated by GH deficiency because, in animal models, it has been demonstrated that CREBBP mutation can influence $\mathrm{GH}$ production both at hypothalamic (interfering with GHRH secretion) and pituitary (disrupting GH gene transcription) level.

Methods: We have studied CREBBP mutation, GH-IGF-1 axis and brain imaging in a patient with RTS.

Results: The molecular study showed a new point mutation in position 3456 (exon 18) of the CREBBP gene. We performed an arginine and clonidine provocative test for $\mathrm{GH}$ secrection. GH peak levels were both $<7 \mathrm{ng} / \mathrm{dL}$. Because of the GH deficiency, we obtained a brain magnetic resonance (MRI) that showed pituitary hypoplasia and Arnold Chiari malformation type 1. A medullary MRI scan showed a double syringomelic cavity (cervical and dorsal) which required urgent surgical treatment.

Conclusions: We describe, for the first time in literature, a case of a patient with RTS, GH deficiency, pituitary hypoplasia, Arnold Chiari malformation type 1 and double syringomielic cavity. We hypothesize that CREBBP gene mutation could be responsible both for GH deficiency and pituitary hypoplasia in our patient. It could be useful to study the GH-IGF-1 axis in more subjects with RTS and CREBBP mutation to understand if GH deficiency could play a role in determing the short stature in RTS.

\section{EPO-126 GH and IGF Physiology \\ A rare familial GH-1 mutation characterized with early infant growth failure \\ Veselin Boyadzhiev' ${ }^{1}$ Vilhelm Mladenov ${ }^{1}$; Violeta lotova ${ }^{1}$; Roland Pfaeffle ${ }^{2}$ \\ ${ }^{1}$ Medical University, Pediatrics, Varna, Bulgaria; ${ }^{2}$ University of Leipzig, \\ Hospital for Children and Adolescents, Leipzig, Germany}

Background: We present a patient with familial short stature running in 3 generations.

Objective and hypotheses: The boy is born after an uneventful pregnancy and normal delivery with weight $3.650 \mathrm{~kg}$ and length $50 \mathrm{~cm}$. The growth delay became obvious after the 6th postnatal month. At presentation, 15 months old, the child weight was on 35 th cdc 2000 centile and height under 3 rd centile (target height at 10th centile). He had normal body proportions, mid-facial hypoplasia and increased subcutaneous fat without system abnormalities. His father was $150 \mathrm{~cm}$ tall with growth deficiency since childhood as well as the grandfather, who was $139 \mathrm{~cm}$ tall and died from myocardial infarction at the age of 42

Methods and results: Initially we found fasting hypoglycemia and IGF-1 of $25 \mathrm{ng} / \mathrm{ml}$ (referent range 83-378). Insulin tolerance and glucagon tests showed marked GH deficiency with highest level $0.94 \mathrm{mIU} / \mathrm{ml}$. Bone age was delayed -9.5 months delay at age 17 months and 12 months delay at 33 months. Brain MRI was without abnormalities. Hormonal analyses of the father showed low basal GH and IGF-1 levels. Genetic analyses revealed a heterozygous splice-site mutation within the first base of Intron 3 of the GH-1 gene (IVS3 $+1 \mathrm{G}->\mathrm{A})$ in the GH-1 gene both in the father and the child, explaining the dominant inheritance of GHD in this pedigree.

Conclusion: We found a rare GH-1 gene mutation which is known to abolish the splice acceptor site and thus leading to a loss of transcription of exon 3 and a synthesis of an abnormal GH-1 isoform that might be responsible for the insufficient secretion of GH from the pituitary. 
Now the child is already on GH treatment and is responding well to a standard dose. Such patients have also been described to develop central hypogonadism at a later age, which warrants targeted follow-up.

\section{EPO-127 GH and IGF Physiology \\ Phenylketonuria and growth hormone deficiency: a case report}

Kosteria Ioanna ${ }^{1}$; Maria Dolianiti' ${ }^{1}$ Efstathia Theodorakopoulou'; Maria Theodorakopoulou'; Kleopatra Soulpi'; Lela Stamogiannou ${ }^{1}$

${ }^{1} \mathrm{P} \&$ A Kyriakou Children's Hospital, 1st Pediatric Department, Athens, Greece; 'Institute of Children Health, Department of Inborn Errors of Metabolism, Athens, Greece

Background: Although growth retardation has been previously described in children with phenylketonuria, it has been attributed to excess phenylalanine restriction. The co-existence of phenylketonuria with growth hormone deficiency has not yet been reported.

Objective: We present the case of a 6-year-old girl diagnosed with classic phenylketonuria since birth and treated with appropriate dietary instructions, including a daily phenylalanine intake of $40-50 \mathrm{mg} / \mathrm{kg}$ at birth, as well vitamin and protein complements. During the child's follow up, a decrease in her growth rate was observed, putting her under the 3rd centile position in normal height curves, despite near normal phenylalanine levels $(5,3 \pm 1 \mathrm{mg} / \mathrm{dl}$, normal values: $1-3 \mathrm{mg} / \mathrm{dl}$ ). Further workup revealed growth hormone deficiency, verified by both insulin (max. GH: 2,88 ng/dl) and L-Dopa (max. GH: 2,25 ng/ dl) stimulation tests, and the child is now under growth hormone replacement treatment. All other imaging and laboratory results were normal.

Conclusion: This is to our knowledge the first report of a child with phenylketonuria and growth hormone deficiency. Given that treated children with phenylketonuria can achieve normal physical growth, any such disturbances should arouse suspicion of other underlying conditions that must be excluded.

\section{EPO-128 GH and IGF Physiology \\ Case report: hypothalamic lipoma (HL) in a 12 years old girl with growth hormone deficiency} Claudia Balsamo; Federico Baronio; Sara Monti; Piero Pirazzoli University of Bologna \& S.Orsola-Malpighi H., Pediatric Clinic

Endocrine Unit, Bologna, Italy

Background: Hypothalamic lipomas are rare congenital lesions of midline region with reported prevalence of about $0.1-0.3 \%$ among all intracranial tumors. They usually are asimpthomatic and rarely need surgery. In children 2 cases with endocrine disturbances as early puberty and obesity were reported. Case report: We report the case of a 12 years old girl previously evaluated in another Centre for severe short stature. Previous evaluation showed normal pituitary function, 46,XX karyotype and no signs of malabsorption or skeletal dysplasia. A MRI of the hypothalamic pituitary region was performed and showed an hypothalamic benign lesion, diagnosed as amarthoma. At our Centre, clinical evaluation shows short stature $(<-3 \mathrm{SD})$ with impaired growth velocity ( $-1.82 \mathrm{SD}$ ), mid-pubertal stage (G3), BMI -1.43 SD and no dysmorphologic features. Bone age is 10.5 yrs. Endocrinological evaluation shows GH deficiency (peak $<5 \mathrm{ng} / \mathrm{ml}$ ) after 2 pharmacological test with IGF-1 in low-normal range (- $0.48 \mathrm{SD})$. The pituitary adrenal, thyroid and gonadal axes show normal function. The MRI scan of the hypothalamic-pituitary region confirms the presence of a lesion on the 3rd ventricle's floor characterized by homogeneous high signal intensity on fast spin echo T1 weighted sequences and low signal intensity on fat saturated T1 weighted images. The lesion is compatible with hypothalamic lipoma.

Conclusions: To our knowledge this is the first case report of GH deficiency associated with HL. We hypothesized that HL in our pt could interfere with normal GHRH release and cause irregular GH secretion. Our case showed that GH axis function should be re-evaluated in pts with hypothalamic lipoma and growth impairment even if first evaluation showed normal GH secretion.

\section{EP0-129 GH and IGF Physiology}

Growth hormone deficiency in a patient with Iysinuric protein intolerance

Evelina Maines; Grazia Morandi; Carlo Forcellini; Claudia Banzato; Francesco Doro; Franco Antoniazzi

University of Verona, Department of Life and Reproduction Sciences, Verona, Italy

Background: Short stature is a common problem among paediatric population but sometimes is the result of a rare and often undiagnosed illness. Lysinuric protein intolerance (LPI) is a rare, autosomal recessive metabolic disorder caused by the defective transport of the cationic amino acids. The clinical presentation of LPI includes not only failure to thrive, but also other findings that suggest a multisystem involvement.

Case report: We report a case of a boy with a history of short stature and laboratory finding of high ferritin and lactic deydrogenase values. After a normal weaning, he suffered from frequent nausea and vomiting, especially eating rich-protein foods. At the first visit to our Clinic he was a 6-year-old boy in good general conditions with a palpable liver and spleen at the inferior costal arch. His weight was $<3$ rd percentile and his height $<3$ rd percentile; Tanner stages were G1, PH1, A0. Initially, we investigated short stature with routine laboratory tests: IGF-1 value was low $(3 \mathrm{nmol} / \mathrm{L})$, while cortisol, HPRL, TSH, fT3, fT4, TGA and EMA values resulted all in the normal range. We performed two provocative tests to measure the growth hormone $(\mathrm{GH})$ peak serum level: both tests (arginine and insuline i.v.) revealed GH values $<10$ $\mathrm{mcg} / \mathrm{L}$. Thereafter we performed plasma and urinary amino-acids determinations. We found lower than normal plasmatic levels of lysine $(62 \mu \mathrm{mol} / \mathrm{L}$, n.v. $115-278 \mu \mathrm{mol} / \mathrm{L})$, ornithine $(13,9 \mu \mathrm{mol} / \mathrm{L}$, n.v. $25-159 \mu \mathrm{mol} / \mathrm{L}), 3-\mathrm{meth}-$ ylhistidine $(1,8 \mu \mathrm{mol} / \mathrm{L}$, n.v. $2-21 \mu \mathrm{mol} / \mathrm{L})$ and arginine $(22,09 \mu \mathrm{mol} / \mathrm{L}, 38-$ $135 \mu \mathrm{mol} / \mathrm{L}$ ) and higher than normal urinary levels of lysine, ornithine and arginine. Moreover bone marrow aspiration revealed hemophagocytosis (HP) in bone marrow. We diagnosed Lysinuric protein intolerance with a classic growth hormone deficiency (GHD) and the patient started GH replacement therapy.

Conclusions: Although the mechanism which correlates GHD and LPI is still not well known, we hypothesize that persistent low-normal levels of arginine and lysine detected in LPI patients may lead to impaired GH secretion.

\section{EPO-130 GH and IGF Treatment \\ The efficiency and safety of replacement therapy by the growth hormone "JINTROPIN $₫$ " in children and adolescents with growth hormone deficiency \\ Said Ismailov'; Yuldus Urmanova²; Gulnara Rakhimova ${ }^{3}$; Barno Nazarova ${ }^{3}$; Feruza Suleymanova ${ }^{3}$; Ravshan Zaripov ${ }^{1}$ Center of Endocrinology, Surgery, Tashkent, Uzbekistan; ${ }^{2}$ Center of Endocrinology, Neuroendocrinology, Tashkent, Uzbekistan; ${ }^{3}$ Center of Endocrinology, Pediatric Endocrinology, Tashkent, Uzbekistan}

Background: Basic rules for indication and progression of treatment are proposed in children with various forms of growth hormone deficiency (GHD). Objective and hypotheses: The purpose of the study evaluated the efficacy of growth hormone (JINTROPIN $囚$ ) in children with GHD.

Methods: This study included 20 patients (4 girls and 16 boys) aged 11,2士0,7 with GHD, who were treated with doses $0,033 \mathrm{mg} / \mathrm{kg} / \mathrm{day}$. Anthropometry, bone age, levels of plasma insulin-like growth factor-I (IGFI), insulinlike growth binding protein-3 (IGFBP-3) and urinary $\mathrm{GH}(\mathrm{uGH})$ have been assessed at baseline and after 3, 6 month.

Results: The height increased from $114,8 \pm 3,3 \mathrm{~cm}(-4,59 \pm 0,2$ height SDS) in the beginning to $116,8 \pm 3,2(-4,1 \pm 0,3$ height SDS $)$ after 3 month and $120 \pm 3,9$ $(-3,9 \pm 0,2$ height SDS $)$ after 6 month of treatment $(p<0,001)$. Bone age increased also from $5,7 \pm 0,7$ years to 7,4 $\pm 1,0$ after 6 month of treatment. Levels of plasma IGF-I, IGFBP-3 and uGH were significantly lower in the start of treatment, than we see tendency to improve the diagnostic markers. The obtained data of laboratory researches of levels of IGF-1, IGFBP-3 and uGH are given in the table.

$\begin{array}{lccc}\text { Diagnostic markers } & 0 & 3 \text { month } & 6 \text { month } \\ \text { IGF-1 (ng/ml) } & 119,8 \pm 12,7 & 228,0 \pm 33,5(p<0,05) & 235 \pm 25,6(p<0,05) \\ \text { IGFBP-3 (ng/ml) } & 688,2 \pm 161,3 & 1090,4 \pm 98,4 & 1091,6 \pm 60,4(p<0,001) \\ \text { uGH }(\mathrm{ng} / \mathrm{ml}) & 0,4 \pm 0,1 & 3,7 \pm 0,8(p<0,05) & 2,9 \pm 0,6(p<0,05)\end{array}$


Adverse effects occurred in 3 cases (15\%), including disk stagnation of the optic nerve ( 2 cases) and pain or paresthesia in place of injection ( 1 cases). Conclusions: The replacement therapy by the growth hormone "JINTROPIN $®$ " is safety and efficiency in patients with GHD. It should be monitoring of levels of IGF-1, IGFBP-3, uGH and estimation of heights.

\section{EP0-131 GH and IGF Treatment}

\section{Scwannoma - a rare secondary neoplasm during growth hormone therapy in a child treated for medulloblastoma}

Tatjana Milenkovic ${ }^{1}$; Dragan Zdravkovic'; Katarina Mitrovic'; Sladjana Todorovic '; Slavisa Djuricic'; ${ }^{2}$ Rade Vukovic ${ }^{1}$

'Institute for Mother and Child Health Care of Serbia, Endocrinology, Belgrade, Serbia; ' Institute for Mother and Child Health Care of Serbia, Pathology, Belgrade, Serbia

Background: Growth hormone (GH) deficiency is common in childhood cancer survivors. It is shown that treatment with $\mathrm{GH}$ increase risk of developing secondary neoplasm (SN) in cancer survivors. Schwannoma is an extremely rare $\mathrm{SN}$ in children treated with $\mathrm{GH}$. According to our knowledge, this is the first reported case of schwannoma in a child treated for medulloblastoma. Results: We report a 13-year-old girl in whom medulloblastoma was diagnosed at the age of 2.5 years. Surgery was followed by chemotherapy and radio therapy. Treatment was finished when she was 3.2 years old. At the age of 7.8 years, the diagnosis of isolated GH deficiency was established and GH

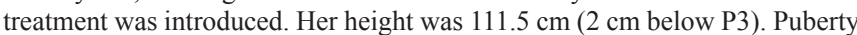
started on time. At the age of 11, she complained of discrete pains in the neck and lumbar spine.

Spine MRI revealed huge, well-defined mass that was in association with the L4-L5 interspinal space. Lesion was ovoid and encapsulated in the retroperitoneal space in the L4-L5 level along the posterior side of right psoas muscle. The tumor demonstrated signs of necrosis. The dimensions of the tumor that was in spinal canal were CC $3 \mathrm{~cm}, \mathrm{LL} 2 \mathrm{~cm}, \mathrm{AP} 1.8 \mathrm{~cm}$ and of that in paraspinal region CC $8.5 \mathrm{~cm}$, LL $4 \mathrm{~cm}$ and AP $4.5 \mathrm{~cm}$. As soon as MRI was done, $\mathrm{GH}$ treatment was stopped. The girl subsequently underwent two operations. Excision of tumor was complete. Histology revealed cellular schwannoma. She underwent normal puberty and menarche appeared when she was 12.8 years old. At the age of 13.3. years, her height was $143.2 \mathrm{~cm}(3 \mathrm{~cm}$ below the 3rd centile), B5, PH5.

Conclusions: Secondary neoplasm during GH treatment is rare, but dramatic event. Still, GH therapy should be considered in any cancer survivor if GH deficiency is confirmed. Close follow up during GH treatment is necessary for early recognition of secondary neoplasm.

\section{EPO-132 GH and IGF Treatment}

\section{Growth hormone deficiency in boy with Noonan syndrome and hypertrophic cardiomyopathy}

\section{Beata Pyrzak}

Medical University of Warsaw, Pediatric and Endocrinology, Warsaw, Poland

Background and aim: Noonan syndrome (NS) is characterized by short stature, congenital heart defect, and developmental delay of variable degree. Growth hormone secretion in NS is usually in normal range. The most frequent cardiac defect is pulmonic valve stenosis (PTPN11 mutation) and hypertrophic cadiomyopathy (in pts without PTPN11 mutation) Treatment of $\mathrm{GH}$ in children with hypertrophic cadiomyopathy (HOCM) dependent of risk factors presented at diagnosis; included heart failure, decreased left ventricular fractional shortening, and increased septal thickness.

Objective: We described 6 y.o boy with severe growth hormone deficiency with NS /RAF1 mutation/ and with hypertrophic cardiomyopathy.

Methods: Boy was born with normal weight and length. In first y.o. the typical phenotype of NS, HOCM and undescended testicles was recognized. During next years of life the decrease of growth velocity was observed. According to Ranke, growth was $-4,2 \mathrm{SDS}$, height gain 3-4 cm per year.

Results: The evaluation of GH secretion showed low spontaneous nocturnal GH secretion $(0.9,3.7,2.2,0.95,1.1 \mathrm{GH} \mathrm{ng} / \mathrm{ml})$, decreased GH secretion after stimulations L-arginin, L-Dopa (0.9, 1.2, 1.8 1.6, $2.2,1.0$ and respectively $1.7,4.2,2.5,0.8,1.0 \mathrm{GH} \mathrm{ng} / \mathrm{ml})$ and decreased IGF1 (73.5 ng/ml). The MRI image reveals a small $(3.6 \times 8 \times 10 \mathrm{~mm})$ normal pituitary gland. Genothyping confirmed RAF1 mutation. Heart ultrasound showed the right and the left outflow tract obstruction septal thickness $>10 \mathrm{~mm}$, ECG left axis deviation, sings of right ventricle hypertrophy, prolonged QT interval and single ectopic ventricular beats.

Conclusion: Treatment of GH in children with HOCM and RAF1 mutation is controversial, in that case though severe GH deficiency we did not determined of $\mathrm{GH}$ treatment.

\section{EPO-133 GH and IGF Treatment \\ Intuitiveness, ease-of-use and dose force in two growth hormone injection devices \\ Anne-Marie Kappelgaard'; Niels Aage Hansen $^{2}$; Franziska Winter ${ }^{3}$; Britta Müller; Marianne Qvist ${ }^{4}$ \\ ${ }^{1}$ Novo Nordisk A/S, Medical Affairs, Global Marketing GHT, Søborg, Denmark; ${ }^{2}$ Novo Nordisk A/S, Device R\&D Strategy \& Process, Hillerød, Denmark; ${ }^{3}$ nstitute for Clinical Research and Development, ikfe CRO GmbH, Mainz, Germany; ${ }^{4}$ Novo Nordisk A/S, Clinical Operations, Insulin and Devices, Søborg, Denmark}

Background: Intuitive, easy-to-use devices with reduced dose force make it easier for patients to self-administer injectable medication and may therefore improve adherence.

Objective and hypotheses: Intuitiveness, ease-of-use and dose force were compared in two growth hormone $(\mathrm{GH})$ injection devices: Norditropin ${ }^{\circledR}$ Fl$\operatorname{exPro}^{\circledR}$ (FP) (Novo Nordisk A/S, Denmark) and Genotropin GoQuick ${ }^{\circledR}$ (GQ) (Pfizer Inc., NY, USA).

Methods: Children treated with GH ( $\geq 6$ months) were randomised to intuitiveness (INT, $\mathrm{n}=32$; mean[SD]age, 13.1[2.1]years) or instruction (INS, $\mathrm{n}=32$; age, 13.4[2.0]years) groups. The INT group received brief instruction on device use; the INS group received full instructions, according to the patient user guide. Time taken to set a $1.5 \mathrm{mg}$ dose and inject into an Eppendorf tube was recorded for each device. Intuitiveness/device preference was subsequently assessed by questionnaire. Maximum dose force was determined at speeds of 4,6 and $8 \mathrm{~mm} / \mathrm{s}$. Dose accuracy and precision were assessed at 0.1 , 0.75 and $1.5 \mathrm{mg}$ doses.

Results: Dose delivery time was significantly lower with FP than GQ (INT, 40 s vs. $66 \mathrm{~s} ; \mathrm{p}=0.001$; INS, $41 \mathrm{~s}$ vs. $48 \mathrm{~s}, \mathrm{p}=0.026$ ). Compared to $\mathrm{GQ}$, FP was rated as the easiest-to-use, most intuitive, and device of overall preference. In the INT group, 97\% reported that they felt confident operating FP without instruction ( $31 \%$ for GQ). Estimated relative dose force for FP was significantly lower than for GQ $(3.6,4.4,5.2 ; \mathrm{p}<0.0001)$ at all injection speeds $(4$, $6,8 \mathrm{~mm} / \mathrm{s}$ ). Dose accuracy at $0.10,0.75$ and $1.5 \mathrm{mg}$ doses was 97,99 and $99 \%$ for FP and 100, 95 and 97\% for GQ, respectively. Dose precision was 2.5, 0.8 and $0.8 \%$ for FP and $11.1,2.6$ and $1.6 \%$ for GQ.

Conclusions: Norditropin ${ }^{\circledR}$ FlexPro ${ }^{\circledR}$ is an intuitive, easy-to-use device with reduced dose force and improved dose precision compared with Genotropin GoQuick $^{\circledR}$. Patients feel confident using Norditropin ${ }^{\circledR}$ FlexPro $^{\circledR}$ without instruction and identify Norditropin ${ }^{\circledR}$ FlexPro ${ }^{\circledR}$ as the device of overall preference.

\section{EPO-134 GH and IGF Treatment \\ Serum insulin like growth factor-1 levels in Indian children on growth hormone therapy \\ Sunil Kumar Kota'; Siva krishna Kota²; Lalit Kumar Meheri; \\ SVS Krishna'; Kirtikumar D Modi' \\ ${ }^{1}$ Medwin Hospital, Endocrinology, Hyderabad, India; ${ }^{2}$ Central Security Hopsital, Anesthesia, Riyadh, Saudi Arabia; ${ }^{M}$ MKCG medical College, Medicine, Berhampur, India}

Background: Insulin-like growth factor-1 (IGF-1) is used for evaluating growth hormone deficiency (GHD).

Objective: We evaluated the effect of recombinant human GH treatment on serum IGF-1 levels in Indian children with GHD over 24 months.

Methods: Patients with short stature were evaluated. The inclusion criteria were a) Height $<3$ rd percentile or 2 standard deviation score (SDS) below national mean b) growth velocity (GV) below the 25 th percentile, c) Serum $\mathrm{GH}$ concentration $<10 \mathrm{ng} / \mathrm{ml}$ during 2 provocation tests. The exclusion criteria were a) Presence of active tumor, systemic disease, chromosomal abnormality, or syndromic illness b) chronic medication other than thyroid or cortisol replacement. Patients were followed up at 3 months interval with auxology evaluation, pubertal staging, bone age determination and IGF-1 assay, 
$\mathrm{HbA1C}$ and fasting blood sugar (FBS) measurement. All patients received 0.3 $\mathrm{mg} / \mathrm{kg} /$ week GH in 7 divided doses subcutaneously daily at night. Patients with multiple pituitary hormone deficiencies received additional substitution therapy. Analysis of variance and unpaired t test were used to calculate differences among groups. $\mathrm{P}$ value $<0.05$ was considered significant.

Results: 32 prepubescent children (M: $F=18: 14)$ with mean age of $7.9 \pm 2.1$ years were enrolled. Maximum height was attained in the first year (110.2 \pm $15.8 \mathrm{~cm}$ ). The auxologic and biochemical parameters of the study group are depicted in table 1. Though there was significant increase in height SDS at all intervals ( $\mathrm{p}, 0.005)$, the IGF-1 SDS at corresponding intervals was $-3.3 \pm 0.9$, $-1.8 \pm 1.1$ and, $-1.3 \pm 1.2$, respectively $(\mathrm{P}>0.1)$. Height $\mathrm{SDS}$ in children with normal IGF-1 SDS did not improve significantly in comparison to children with IGF-1 SDS $<-2$ at 2 years Bone age advancement, the occurrence of puberty, and FBS, HbA1C did not change significantly during therapy. One girl at 12 months and 2 girls at 24 months entered puberty.

Conclusion: Changes in serum IGF-1 SDS may not be a reliable marker for responsiveness to GH therapy in Indian children.

\begin{tabular}{lccc|} 
& Time frame & & \\
Parameters (mean \pm SD) & Baseline & 12 months & 24 months \\
\hline Height (cms) & $97.8 \pm 13.4$ & $110.2 \pm 15.8^{*}$ & $118.1 \pm 16.3^{*}$ \\
\hline Height SDS & $-5.2 \pm 1.3$ & $-3.9 \pm 1.3^{*}$ & $-3.2 \pm 1.4^{*}$ \\
\hline BMI (Kg/m2) & $14.2 \pm 1.3$ & $13.9 \pm 2.2$ & $16.0 \pm 2.9$ \\
BMI SDS & $-1.3 \pm 0.5$ & $-1.1 \pm 1.6$ & $-0.9 \pm 1.1$ \\
Growth Velocity SDS & $-2.2 \pm 1.2$ & $3.2 \pm 1.7^{*}$ & $2.0 \pm 1.4^{*}$ \\
\hline Peak GH (ng/ml) & $0.9 \pm 1.1$ & - & - \\
\hline Serum IGF-1 (ng/ml) & $28.9 \pm 17.3$ & $134.2 \pm 113.8^{*}$ & $189.9 \pm 138.2^{*}$ \\
IGF-1 SDS & $-3.3 \pm 0.9$ & $-1.8 \pm 1.1$ & $-1.3 \pm 1.2$
\end{tabular}

\section{EPO-135 GH and IGF Treatment}

\section{Height velocity targets for the first year response to recombinant human growth hormone (rhGH) treatment in pre-pubertal growth hormone deficiency (GHD) children - data from single centre in China}

Zhe Su'; Hua-Mei Ma'; Si-Nian Pan'; Min-Lian Du

${ }^{1}$ The First Affiliated Hospital of Sun Yat-Sen University, Department of Pediatrics, Guangzhou, China; ${ }^{2}$ The Third Affiliated Hospital of Sun YatSen University, Department of Pediatrics, Guangzhou, China

Background: One strategy for optimizing growth hormone (GH) treatment is to develop mathematical predictive model as the authors have done before. However, it is still uncertain about how best to interpret and evaluate a growth response in China. Such height velocity target is needed for individualizing growth hormone dose when using a predictive model.

Objective and hypotheses: To explore the height velocity targets for the first year response to recombinant human growth hormone $(\mathrm{rhGH})$ treatment in pre-pubertal Growth Hormone Deficiency (GHD) children in China.

Methods: Analyze retrospectively the height velocity data before (HV0) and one year after $(\mathrm{HV} 1)$ rhGH treatment in physiologic dose $(0.7 \mathrm{u} / \mathrm{kg} / \mathrm{w})$ in prepubertal GHD children above 3 year old who were diagnosed from Jan, 2000 to Dec,2009 in our hospital. There were totally 342 records of HV0. According to the peak $\mathrm{GH}$ value in $\mathrm{GH}$ provocative test, the patients who were included for calculation of HV1 were divided into GHD-1 group $(<7 \mathrm{ng} / \mathrm{ml}, 140$ patients) and GHD-2 group ( $7 \mathrm{ng} / \mathrm{ml}-9.9 \mathrm{ng} / \mathrm{ml}, 33$ patients $)$.

Results: Within every bone age group, GHD-1 group has significantly higher HV1 than GHD-2 group $(\mathrm{p}<0.05), 11.0(10.5-11.5) \mathrm{cm} / \mathrm{y}(\mathrm{n}=34)$ vs 9.9 $(9.1-10.8) \mathrm{cm} / \mathrm{y}(\mathrm{n}=6)$ when bone age was less than 3 years, $10.4(9.8-10.9)$ $\mathrm{cm} / \mathrm{y}(\mathrm{n}=48)$ vs $8.8(8.3-9.2) \mathrm{cm} / \mathrm{y}(\mathrm{n}=8)$ when bone age was between 3 to 5 years, $9.5(9.1-9.9) \mathrm{cm} / \mathrm{y}(\mathrm{n}=58)$ vs $8.5(8.0-9.1) \mathrm{cm} / \mathrm{y}(\mathrm{n}=19)$ when bone age was between 6 to 10 years. The mean HV1 of GHD-2 was very close to the 25th percentile of GHD-1 group. The 5th percentile HV1 of GHD-1 group was only $2.5 \mathrm{~cm} / \mathrm{y}$ to $3.3 \mathrm{~cm} / \mathrm{y}$ higher than HV0. They both were significantly higher than HV0.

Conclusions: It is more reasonable to evaluate growth response in pre-pubertal GHD patients according to bone age group when the case number was limited. It can be considered a "poor" response when HV1 was below the 25 th percentile of GHD-1 group. Nearly $50 \%$ of the GHD-2 group may need higher $\mathrm{GH}$ dose.
EP0-136 GH and IGF Treatment

\section{A five-year-old girl with Turner syndrome} associated with juvenile rheumatoid arthritis and small for gestational age on the growth hormone treatment background

Maia Rekhviashvili'; David Metreveli'; Revaz Morgoshia'

'The Family Medicine Traning Clinical Center of "VERE XXI",

Department of Physical and Sexual Devolopment of Children and Adolescent, Tbilisi, Georgia; ${ }^{2}$ David Metreveli Clinical Center, Tbilisi, Georgia

Background: In pediatrics it is very important to determine and to differentiate various forms of the growth disorders, which may constitute a manifestation of totally different diseases. Furthermore growth retardation has a negative impact on persons self-realization in terms of self-esteem, social restriction and stigmatization.

Objective and hypotheses: We describe a 5 year old girl. The reason for the first presentation at our pediatric endocrinology department was because of her short stature (height Standard deviation score $-5,01$ ) at five years of age. Bone age was retarded by 1 year according to Greulich \& Pyle.

Methods: Physician examination was notice High palate, widely standing nipples, "Pterigium coli", wrinkle on the neck, "Cubitus Valgus", Stigmatic years and nails, partial restriction of movement caused by the knee joints deformation and foot curvature. Target height was $159,5 \mathrm{~cm}$. In the anamnesis, the child had frequent year ache since early childhood. She was born in the 40th week, weight $2300 \mathrm{~g}$, length $48 \mathrm{~cm}$, through pathological pregnancy and delivery, in asphyxia and muscles hypotenuses. The cyto-genetical analyses stated the Turner-Ulrich Syndrome diagnosis (45XO). Since the age of 2 the patient has been treated on the basis of juvenile rheumatoid arthritis diagnosis. Consequently she has been taking corticosteroids for 2, 6 years, medicinal exercises and manual therapy. The body proportions was normal ([sitting Ht]/ [body Ht] , hypothyroidism was excluded. At the moment of applying she was observed juvenile rheumatoid arthritis with clinical remission.

Results: Growth hormone appointed has improved the growth rate and general condition- she became more sociable. For the 6 month of treatment the height SDS reduced for $(-5,01)$ to $(-4,51)$.

Conclusions: We can conclude, that growth hormone treatment resulted a significant improvement in height Standard deviation score and general condition and is necessary with different causes growth disorders.

\section{EPO-137 GH and IGF Treatment}

\section{The efficasy and safety of new soluble form of somatropin preparation in children with GHD}

Tatiana Shiryaeva; Elena Nagaeva; Natalia Volevodz;

Olga Bezlepkina; Olga Chikulaeva; Valentina Peterkova

Endocrinology Science Center, Pediatric, Moscow, Russian Federation

Background: Outcome of GH-therapy depends on patient's compliance, determined by form and convenience of GH-preparations (GHP). New soluble form of GHP was innovated in Russia.

Objective and hypotheses: To estimate the efficacy and safety of new form of GHP for everyday use.

Methods: 42 patients ( 31 boys) from 4 to 12 years old (average chronological age $9,4 \pm 2,3$ years) with GHD, average bone age delay $3,1 \pm 1,9$ years, average height SDS (-) 2,20 $\pm 1,28$ (at study start) were included in the study. Previous to study inclusion all the children were treated with different GHP during 2-2,5 years. The patients were randomized in 2 group: first group was treated with study preparation (SP) in daily dose $0,033 \mathrm{mg} / \mathrm{kg}$ during 3 months and second group was under treatment with comparison preparation (CP), lyophilized GH for solution prepare, in the same way. Follow-up 9 months all the patients were treated with SP.

Results: During the first 3 months of treatment the statistically significant increase of measured height was registered (group $1 \Delta$ HSDS $0,27 \pm 0,13$, $\mathrm{p}=0,004$; group $2 \Delta \operatorname{HSDS} 0,30 \pm 0,16, \mathrm{p}=0,003$ ). The absence of statistically significance in height gain $(p=0,235)$ and HSDS $(p=0,90)$ between the groups pointes to the same growth efficacy of SP and CP. During follow-up 9 months treatment the increasing of height, HSDS and IGF-1 level indicate on stabile growth impact. Any significant changes in clinical blood and biochemistry analyses as well as fT4, cortisol and prolactine levels were not registered. Any adverse events closed with GH-therapy were not described. In the course of the study none of patients discontinued treatment. At the end of the study all of them made choice of new soluble GH form.

Horm Res 2012;78(supp1 1) 285 
Conclusions: The therapy with new soluble form of GHP is defined by satisfactory characteristics of efficacy and safety. The patient's compliance was much better under the treatment with soluble GHP.

\section{EPO-138 GH and IGF Treatment}

\section{GH postoperatory treatment in a 16-year-old} boy with craniopharyngioma-case report

\section{Andra Iulia Loghin'; Ilinka Grozavu'; Alexandru Florescu';}

Liliana Moisii2; Petronela Aancute'; Corina Galesanu'

${ }^{1}$ University of Medicine and Pharmacy "Gr. T. Popa", Endocrinology, lasi, Romania; ${ }^{2}$ University of Medicine and Pharmacy "Gr. T. Popa",

Radiology, lasi, Romania

Background: Craniopharyngiomas are the second cause of hypopituitarism in children.The manifestations at diagnosis are nonspecific:headache, visual impairment, polyuria, polydipsia, growth retardation and weight gain.Multiple pituitary deficiencies and diabetes insipidus are frequent.

Objective and hypotheses: The benefits of GH therapy in a case of growth disturbance, obesity and dyslipidemia due to craniopharyngioma.

Methods: Case of a 16-year-old boy with polyuro-polydipsic syndrome, severe headache, bitemporal hemianopsia, symptoms aggravated in the last 2years. Clinical examination:height $=145 \mathrm{~cm}(-4 \mathrm{DS})$, weight $=45 \mathrm{Kg}(-1.8 \mathrm{DS})$, abdominal circumference $=89 \mathrm{~cm}(+3 \mathrm{DS})$, no puberty signs.Laboratory findings revealed diabetes insipidus and dyslipidemia.Hormonal profile diagnosed somatotroph deficiency $(\mathrm{GH}=0.1 \mu \mathrm{IU} / \mathrm{ml} ; \mathrm{IGF} 1=55.7 \mathrm{ng} / \mathrm{ml})$, tireotroph deficiency $(\mathrm{TSH}=6.9 \mu \mathrm{IU} / \mathrm{ml} ; \quad \mathrm{FT} 4=6.3 \mathrm{pmol} / 1)$, gonadotroph deficiency $(\mathrm{FSH}=0.5 \mathrm{mIU} / \mathrm{ml} ; \mathrm{LH}<0.1 \mathrm{mIU} / \mathrm{ml}$; testosterone $=0.02 \mathrm{ng} / \mathrm{ml}$ ) and corticotroph deficiency (cortisol=22.6ng/ml).Pituitary X-Ray revealed a calcified intrasellar expansive process, confirmed by CT:heterogenous, predominantly cystic $29.4 / 29 / 57 \mathrm{~cm}$ tumour with calcifications, suprasellar intracerebral extension, mass effect on anterior cerebral arteries, optic chiasma and hypothalamus, invasion of the 3rd ventricle.Bone age was delayed (12years 9months). Medical treatment: desmopressin acetate $(120 \mu \mathrm{g} / \mathrm{d})$, prednisone $(5 \mathrm{mg} / \mathrm{d})$ and LT4 $(100 \mu \mathrm{g} / \mathrm{d})$.Neurosurgery:subtotal tumour resection, optical nerves and chiasma decompression, followed at 2 months by Gamma Knife stereotaxical radiosurgery. $\mathrm{GH}$ therapy $(1 \mathrm{mg} / \mathrm{d})$ was initiated after 3 months.

Results: After 12 months of GH therapy:height $=157 \mathrm{~cm}(-2.5 \mathrm{DS})$, weight $=58(-$ 0.6DS); normal lipidic profile; no signs of tumour recidive on IRM.

Conclusions: Postoperative rhGH treatment has a benefic influence on growth rate (growth velocity $=12 \mathrm{~cm} /$ year), BMI and lipidic metabolism.Lifelong follow-up is crucial due to the risk of recurrence and the need for ongoing hormone replacement therapy.

\section{EPO-139 GH and IGF Treatment}

\section{Evaluation of the diagnosis and assessment of response to treatment in patients with growth hormone}

\section{Yilmaz Kor; Mehmet Keskin}

Gaziantep University, Faculty of Medicine, Pediatric Endocrinology and Metabolism, Gaziantep, Turkey

Background: This study was performed for contribution to literature by evaluation of children who were diagnosed as growth hormone deficiency and treated in the Department of Pediatric Endocrinology and Metabolism. Objective and hypotheses: To determine the factors associated growth velocity during the growth hormone therapy.

Methods: One hundred and forty-two children who were treated with growth hormone were evaluated retrospectively. 142 cases at least one year, 73 cases at least two years, 25 cases at least three years, 9 cases at least four years and two cases at least five years were followed. Body height, SDS of height, BMI, age at the beginning of medical therapy, bone age, pituitary MRG, height age, parents' height, midparental height, SDS of midparental height, IGF-1 level, IGFBP-3 level, responses to the growth hormone stimulation tests and gaining height and SDS of height were recorded in certain periods during $\mathrm{GH}$ therapy. Dose of $\mathrm{rhGH}$ was applied between $0.2-0.25 \mathrm{mg} / \mathrm{kg} /$ week in our department.

Results: The average age of the patients was $9.97 \pm 3.22$ year. SDS of height was found $-3.46 \pm 1.18$ and delta SDS $-2.49 \pm 1.39$, SDS of midparental height $0.96 \pm 0.72$, bone age $6.69 \pm 3.13$ year, height age $6.50 \pm 2.77$ year and BMI $17.05 \pm 3.43 \mathrm{~kg} / \mathrm{m}^{2}$ before treatment. Height gaining was $11.42 \pm 2.89 \mathrm{~cm} /$ year in the first six months, $8.55 \pm 2.48 \mathrm{~cm} /$ year in the second six month. SDS of height gaining was $0.80 \pm 0.45$ after the first year of the medical therapy. Patients with hypothyroidism had better responses to the medical therapy. We found that the patients had better gaining SDS of height if there was severe short stature or delta SDS under -2.5 or being treated at prepubertal age. Conclusion: This study showed that growth velocity during the growth hormone therapy was associated with multiple factors.

\section{EPO-140 GH and IGF Treatment}

\section{Postprandial hyperglycaemia corrected by IGF- 1 (Increlex $\otimes$ ) in Laron syndrome}

\section{Albane Simon'; Hanane Latrech'; Jacques Beltrand';}

Jean-Claude Souberbielle ${ }^{4}$; Ghizlane Belmejdoub2; Michel Polak ${ }^{3}$

'Versailles Hospital, Department of Paediatrics, Le Chesnay, France; ${ }^{2}$ Military Hospital Mohammed V, Endocrinology and Diabetology,

Rabat, Morocco; ${ }^{3}$ Necker Enfants-Malades Hospital, Paediatric

Endocrinology Gynaecology and Diabetology Unit, Paris, France;

${ }^{4}$ Necker Enfants-Malades Hospital, Department of Physiology, Paris, France

Background: Laron syndrome is caused by a mutation in the growth-hormone $(\mathrm{GH})$ receptor and manifests as insulin-like growth factor-1 (IGF-1) deficiency, severe short stature, and early hypoglycaemia. We report a case of Laron syndrome with frequent postprandial hyperglycaemia, a finding that has not been emphasized previously.

Objective and hypotheses: Postprandial hyperglycaemia is due to chronic IGF-1 deficiency and is reversed by IGF-1 replacement therapy.

Methods: A Moroccan girl referred for short stature at 7 years and 8 months of age had dwarfism (height, $78 \mathrm{~cm}$ [-9 SDs]; weight, $10 \mathrm{Kg}$ [-4 SDs]), hypoglycaemia, and truncal obesity. Her serum IGF-1 level was very low and her baseline serum GH level was elevated to $47 \mathrm{mIU} / \mathrm{L}$. Molecular analysis showed a homozygous mutation in the GH receptor gene.

Results: Continuous glucose monitoring before treatment showed asymptomatic hypoglycaemia with marked postprandial hyperglycaemia $(2.5 \mathrm{~g} / \mathrm{L})$. Treatment with the recombinant human IGF-1 (Mecasermin, Increlex $®$ ) was started. The blood glucose profile improved with $0.04 \mu \mathrm{g} / \mathrm{Kg} /$ day and returned to normal with $0.12 \mu \mathrm{g} / \mathrm{Kg} / \mathrm{day}$.

Conclusions: Postprandial hyperglycaemia is a metabolic consequence of chronic IGF-1 deficiency. The beneficial effect of IGF-1 replacement therapy may be ascribable to improved postprandial transfer of glucose. * : A.S. and H.L. contributed equally to this work and should be considered as joint first author.

\section{EPO-141 GH and IGF Treatment}

\section{GH-deficit in a patients with interstitial deletion of $4 \mathrm{q}$ chromosome: a case report}

Emanuela Scarano'; Marco Seri'; Federica Tamburrino'; Annamaria Perri'; Benedetta Vestrucci'; Francesca Montanari'; Laura Mazzanti

${ }^{1}$ Rare Disease and Auxology Unit, Department of Pediatrics, S.OrsolaMalpighi Hospital, University of Bologna, Bologna, Italy; ${ }^{2}$ Clinical Genetics, S.Orsola-Malpighi Hospital, University of Bologna, Bologna, Italy

Background: Variable clinical presentations of patients with chromosomally detected deletions in the long arm of chromosome 4 have been reported. Objective and hypotheses: The aim of this study was to evaluate GH therapy results in a pt with del 4q.

Case report:We report a girl referred to our center at 8 yrs for dysmorphic features and MR. At the auxoloical evaluation: $\mathrm{H}<<3$ rd centile, $\mathrm{TH}>3$ rd centile, W 50th centile for staturale age, OCP 97th centile. At the psysical evaluation: short stature, mild MR, delayed speech, facial anomalies (epicanthal fold, micro-retrognathia, pear shaped nose), pectus excavatum, scoliosis, hypopigmentation of the linea alba, short hands with brachydactyly, short feet, sparse hair. No evidence of internal malformations. GH deficiency was diagnosed and she was treated with GH reaching a final height of $141.8 \mathrm{~cm}$ $(<<3$ rd centile). At the array CGH del 4(q13.2q21.22) de novo was diagnosed. $4 \mathrm{q}$ deletions are classified in proximal, interstizial and distal deletions. The location of the deletion breakpoints and the size of the deletion are variable. The phenotype associated with distal $4 \mathrm{q}$ deletion is generally less severe than in larger or more proximal deletions. The most common anomalies reported 
are craniofacial, digital, skeletal, cardiac and developmental delay. GH deficit generally is not studied in these patients.

Conclusions: Our patient showed facial anomalies, digital anomalies, scoliosis and MR. We know that the deletion 4(q13.2q21.22) involves a large number of genes: in particular PRKG2, RASGEFIB genes and BMP3 and genes encoding for chemokine, glycosyltransferase proteins and growth factor proteins. BMP3 haploinsufficiency appears to be causative of peculiar skeletal abnormalities including brachydactyly and probably is responsible of the lack of response to GH-therapy. Other studies are necessary for a precise genotype-phenotype correlation.

\section{EPO-142 GH and IGF Treatment \\ Adult height after growth hormone treatment in Chinese children with growth hormone deficiency \\ Sinian Pan ${ }^{1}$; Huamei Ma'; Shunye Zhu'; Zhe Su; Yanhong Li'; Minlian $D u^{2}$ \\ ${ }^{1}$ Third Affiliated Hospital of Sun Yat-sen University, Pediatrics, Guangzhou, China; ${ }^{2}$ First Affiliated Hospital of Sun Yat-sen University, Pediatrics, Guangzhou, China}

Background: Recombinant human growth hormone (rhGH) is expensive to most growth hormone deficiecy(GHD) children in China.

Objective and hypotheses: To evaluate the effect of $\mathrm{GH}$ on the adult height (AH) in Chinese children with GHD.

Methods: This study included 36 children (20 boys and 16 girls) with GHD, who were treated with subcutaneous injections of $\mathrm{rhGH} 0.7 \mathrm{IU} /(\mathrm{kg})[0.23 \mathrm{mg} /$ $(\mathrm{kg})$ ] daily before sleep for 1.1 to 7.3 years. Regular follow-up observation was taken after treatment being discontinued. $\mathrm{AH}$ was defined as a bone age over 16 years for boys and over 14 years for girls or the growth rate less than $2 \mathrm{~cm} /$ year. AH and the factors determing AH were also evaluated.

Results: AH standard deviation score (AHSDS) were $(-1.36+/-0.41)$ in boys and $(-1.13+/-0.53)$ in girls. $85 \%(17 / 20)$ of the boys and $56.2 \%(9 / 16)$ of the girls achieved AH height which was comparable to or above their genetic target height(THt). AHSDS was not significantly different from THt standard deviation score (THtSDS) in boys and girls $(\mathrm{P}>0.05)$. AHSDS in boys was negatively correlated with the age at the initiation of therapy and was positively correlated with HtSDS at the initiation of GH therapy, HtSDS at initiation of puberty and duration of therapy. Stepwise regression analyse indicated that HtSDS at initiation of puberty was the variable with the greatest identified influence on AHSDS in boys $(\mathrm{F}=43.46, \mathrm{P}=0.002)$. But AHSDS in girls was not correlated with the above variables. There was no difference in AHSDS between children with isolated GHD and those with multiple hormone deficiencies $(\mathrm{P}>0.05)$.

Conclusions: $\mathrm{GH}$ improves $\mathrm{AH}$ of children with GHD. Height at the initiation of puberty is the most significant determining factor for the long-term efficacy. Hence, it is important that the diagnosis should be made and treatment be initiated as early as possible to afford children with GHD the opportunity to make up much of their height deficit before puberty. Adequate dosage of GH should be used for the children taking initial treatment at puberty to attain satisfactory $\mathrm{AH}$.

\section{EPO-143 GH and IGF Treatment \\ First- year growth response to growth hormone treatment as a predicting factor for the long- term response in children \\ Efstathia Theodorakopoulou'; Maria Dolianiti' ; \\ Maria Theodorakopoulou'; Ioanna Polychronit'; Aspasia Foteinou²; Lela Stamogiannou ${ }^{1}$ \\ ${ }^{1} \mathrm{P}$ \& A Kyriakou Children's Hospital, 1st Pediatric Department, Athens, Greece; ${ }^{2} \mathrm{P}$ \& A Kyriakou Children's Hospital, Department of Microbiology, Athens, Greece}

Background: Growth response on treatment is influenced by several variables. It is empirical knowledge that first year growth response is an indicator of the response in subsequent years.

Objective and hypotheses: The aim of the present study is to observe if the first year growth response to GH could predict long-term growth response to treatment and whether sex influences final height. Population and methods: We reviewed the files of 50 children with GHD (boys 30, girls 20 mean age:
14,8 years ) treated at the endocrinology department of our clinic and had concluded their therapy.

Results: Measurements of growth response were obtained one year after the initiation of $\mathrm{GH}$ replacement. The hypothesis of the present study was confirmed, we found that children with high first year HV ( $>97$ th percentile) compared to those with low first year HV $(<10$ th percentile) better achieved their target height $(\mathrm{p}$ value $=0,003)$. Moreover boys achieved their target height by $65 \%$ compared to girls who achieved their target height by $75 \%$ and the result is statistically significant by $5 \%$.

Conclusions: Our data confirmed that first year height velocity can be used as an aid in predicting the final height in children treated with $\mathrm{GH}$.

\section{EPO-144 GH and IGF Treatment \\ Seasonal variation of growth response in children with idiopothic growth hormone deficiency \\ Maria Dolianiti; Efstathia Theodorakopoulou; Maria Theodorakopoulou; Ioanna Polychroni; Dimitrios Delis; Lela Stamogiannou \\ P \& A Kyriakou Children's Hospital, 1st Pediatric Department, Athens, Greece}

Background: It is known that longitudinal growth of children exhibits seasonality with maximum growth rate during spring and summer ( in both healthy children and children with GHD).

Objective and hypotheses: The aim of the present study is to investigate whether the growth response to GH therapy in children with GHD is affected by the season when treatment is started. Population and methods: We reviewed the files of 50 children with GHD (boys 30, girls 20 mean age: 14.8 years ) treated at the endocrinology department of our clinic and had concluded their therapy.

Results: Patients were divided in two subgroups, those who began GH replacement during the months of May to October (group A) and those who started from November to April (group B). Height velocity (HV) was enhanced after the initiation of GH therapy in all patients. Patients from group A achieved their target height at $72 \%$ vs $58 \%$ in group B and the result is statistically significant by $5 \%$. Concerning the first year $\mathrm{HV}$ we found no statistical difference between the two subgroups ( $\mathrm{p}$ value $=0,653$ ).

Conclusions: Our data suggest that there is a relation between seasonality and $\mathrm{GH}$ response to treatment. Further confirmation is needed in a larger group of individuals before any conclusions can be drawn.

\section{EPO-145 GH and IGF Treatment}

\section{Short-term metabolic effects of GH treatment in SGA and GH-deficiency children} Sonya Galcheva; Veselin Boyadzhiev; Vilhelm Mladenov; Mariana Moskova; Diana Nedyalkova; Violeta lotova UMHAT, Department of Paediatrics and medical genetics, Varna, Bulgaria

Background: Growth hormone $(\mathrm{GH})$ treatment has effects not only on the linear growth in small for gestational age (SGA) and GH-deficiency children, but also on their glucose, lipid and protein metabolism.

Objective and hypotheses: To evaluate the short-term $\mathrm{GH}$-induced changes in linear growth, body weight and metabolic variables in children born SGA and those with isolated GH deficiency or multiple pituitary deficiencies.

Methods: We included 19 children on GH treatment $(73.7 \%$ boys, $82.4 \%$ prepubertal, mean age $7.91 \pm 4.76$ years at start, age range $2-16$ years) divided into three groups: 5 children $(26.3 \%)$ were born SGA, 6 children $(31.6 \%)$ had an isolated GH deficiency and 8 children $(42.1 \%)$ were with a multiple pituitary deficiency. The mean treatment duration for all children was $6.3 \pm 4.8$ months. Anthropometric measurements and bone age evaluation were performed. Serum levels of insulin-like growth factor-I (IGF-I), fasting BG, HbA1c, total cholesterol, triglycerides (TG), HDL- and LDL-cholesterol concentrations were determined.

Results: There was a significant increase in children's body height and weight at the end of this short-term study treatment $(18.7 \pm 11.4$ vs. $21.1 \pm 13.4 \mathrm{~kg}$, $\mathrm{p}=0.006 ; 105.1 \pm 25.4$ vs. $109.8 \pm 26.2 \mathrm{~cm}, \mathrm{p}<0.001)$. Serum IGF-I concentrations increased significantly in all participants $(\mathrm{p}=0.04)$ and its post-treatment levels correlated with $\mathrm{GH}$ daily dose $(\mathrm{r}=0.867, \mathrm{p}<0.001)$, treatment duration $(r=0.596, p=0.015)$, children's post-treatment height $(r=0.832, p<0.001)$ and 
weight $(\mathrm{r}=0.872, \mathrm{p}<0.001)$. We found a decrease in total cholesterol, TG and LDL-cholesterol concentrations, although the changes did not reach a statistical significance ( $p>0.05$ for all). HbAlc was not significantly changed at the end of the observed treatment interval ( $5.1 \pm 0.4$ vs. $5.2 \pm 0.4 \%$, p $>0.05)$. Comparing children between groups, we did not determine any significant differences in the investigated metabolic outcomes related to the GH treatment. Conclusions: GH treatment in children results in a better metabolic profile with similar responses irrespective of the diagnosis.

\section{EPO-146 GH and IGF Treatment}

\section{The link between birth weight and growth hormone treatment response in patients with growth hormone deficiency}

Alice Albu' ; Alina Roman'; Carmen Barbu'; Simona Fica'

${ }^{1}$ UMF Carol Davila, Endocrinology, Bucharest, Romania; ${ }^{2}$ Elias

Hospital, Endocrinology, Bucharest, Romania

Background: The relationship between intrauterin growth and postnatal growth is very clearly demonstrated in children born small for gestational age. Few previous studies suggested that this relationship could be also important in other categories of children.

Objective and hypotheses: The aim of our work is to study the relationship between birth weight and the response to GH treatment in children with isolated and idiopathic GH deficiency.

Methods: We retrospectively studied 73 children (mean age 8,47 $\pm 3,54$ years at diagnosis, mean birth weight SDS $-0,24 \pm 1,1$ ) treated with rhGH (mean dose $0,038 \pm 0,011 \mathrm{mg} / \mathrm{kg} /$ day) for $2,25 \pm 1,67$ years. GH deficiency was diagnosed based on GH level below $10 \mathrm{ng} / \mathrm{ml}$ during two provocative tests (insulin tolerance test and clonidine test). The response to treatment was considered to be good if the mean improvment in height SDS/ year of treatment was $\geq 0,3$ and poor if the mean improvment in heightSDS/year of treatment was $<0,3$.

Results: We found that children with birth weight $\mathrm{SDS}<0$ had a significantly lower height SDS at initial evaluation and a significantly lower improvment in SDS for height/year of treatment comparing with those with birth weight $>0$ $(0,53 \pm 0,1$ vs $0,83 \pm 0,19, p<0,05)$. Patients with a good response to treatment had higher birth weight, higher mean parental height SDS and higher peak GH response during tests. Logistic regression analysis showed that a good response to treatment was independently associated with higher birth weight $(p<0,05)$ even after adjustment for mean parental height SDS, age at treatment initiation and peak $\mathrm{GH}$ response to provocative tests.

Conclusions: In children with isolated GH deficiency the response to rhGH treatment could be in relationship with birth weight, possibly as a consequence of intrauterin influences in postnatal life.

\section{EPO-147 GH and IGF Treatment}

\section{Isolated growth hormone deficiency (GHD) presenting with hypoglycemic seizures: a clinical case}

Maria Piera Ferrarello; Giuseppe Cannalire; Alessandra Musio; Valentina Donghi; Chiara Maria Damia; Gisella Garbetta;

Gabriella Pozzobon; Berardo Di Natale; Giuseppe Chiumello

IRCCS Ospedale San Raffaele, pediatric Unit, Milano, Italy

Background: Isolated Growth Hormone deficiency (GHD) in the first months of life may present with seizures due to hypoglycemia.

Objective and hypotheses: To analyze hormonal status in a child with hypoglycemic seizures.

Methods: F.C. , female, arrived to our attention at 12 months of age.

Results: During the first months of life she reported poor height and weight growth. The first seizure not associated with fever occurred at 11 months of age. During hospitalization severe height retardation was found, associated with a peculiar phenotype (macrocrania, frontal bossing, depressed nasal bridge). Karyotype, screening of celiac disease, thyroid hormones and neurological examination were normal. IGF1 levels were very low $(<25 \mathrm{ng} / \mathrm{ml})$. A second seizure occurred at home at the age of 12 months. Blood glucose profile showed fasting hypoglycemia (DTX $45 \mathrm{mg} / \mathrm{dl}$ ). The child arrived to our attention for suspected panhypopituitarism. The tests performed showed normal adrenal and thyroid function. GH and cortisol levels were measured during hypoglycemia: their values were indicative of isolated GHD (GH: 0.2 $\mathrm{ng} / \mathrm{ml}$; cortisol: $139 \mathrm{ng} / \mathrm{ml}$ ). IGF1 and IGFBP-3 levels revealed pathologi- cal values. We performed dexamethasone stimulation test for GH which confirmed GHD (peak of $0.3 \mathrm{ng} / \mathrm{ml}$ ). Developmental quotient was normal. Brain Magnetic Resonance showed no abnormalities of hypothalamic-pituitary region, except a small pituitary gland. Analysis of genes involved in panhypopituitarism (POU1F1, PROP1, HESX1, LHX3) was normal. During follow-up, facial childish appearance reduced and muscle tone improved. She presented a good height gain $(+17.9 \mathrm{~cm}$ during two years of therapy) and normalization of IGF-1 blood levels.

Conclusions: F.C. is affected by isolated GHD. Considering clinical presentation of GHD, which may be variable in the first months of life, ranging from absent to more severe nonspecific symptoms, particular attention is required in patients with hypoglycemia.

\section{EPO-148 GH and IGF Treatment \\ Experience with growth hormone "rastan" for the treatment of growth retardation in patients with intra-uterine growth retardation}

Yulia Samoylova; Oxana Oleynik; Maria Novoselova; Ilya Magerya; Elena Kharlamova

Siberian State Medical University, Endocrinology and Diabetology,

Tomsk, Russian Federation

Background: At a delay of intra-uterine development associated with a growth inhibition in postnatal the period, up to $60 \%$ of children with growth inhibition have insufficient secretion Growth hormone or reduced level IGF-1.

Objective and hypotheses: Work to study efficiency and safety of application recombinant a hormone of growth "Rastan" within 18 months at children with a delay of intra-uterine development and expressed growth inhibition in postnatal the period. The group of the surveyed included 12 persons ( 4 girls and 8 boys, are elderly 6,6 $\pm 0,9$ years) with Delay of pre-natal development, prepubertatis age (on Tanner 1 item) with the excluded deficiency of a hormone of growth, comparison group children have made, comparable inclusions by all criteria, but not receiving replaceable therapy Growth hormone. Method: Indicators of efficiency of therapy were: dynamics of growth rates (absolute and SDS), bone maturing, change of the hormonal status. Treatment included hypodermic daily injections Growth hormone (Rastan, manufacture of "Farmstandart", Russia) in a dose of $0,033 \mathrm{mg} / \mathrm{kg}$ in the evening.

Results: Following the results of 18 months of therapy Growth hormone the increase in absolute growth on $11,6 \pm 2,7 \mathrm{sm}(\mathrm{p}<0.05)$, increase SDS of growth rate with initial-1,9 $\pm 0,9$ to $8,2 \pm 2$ for 18 months on the average on $2,8 \pm 0,93$

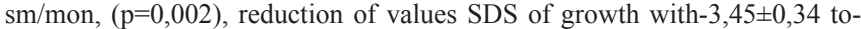
$2,10 \pm 0,67(\mathrm{p}=0,028)$. The maximum acceleration of rates of increase was observed within the first 4 months of treatment, some decrease in indicators of an absolute increase further was marked. The parity of bone/chronological age initially made $0,68 \pm 0,34$, whereas after treatment $0,79 \pm 0,43(\mathrm{p}=0,021)$. In comparison group studied indicators had no authentically significant positive dynamics. The serious undesirable phenomena wasn't registered during 18-month's treatment Growth hormone

Conclusions: Therapy Growth hormone at children with a pre-natal arrest of development and postnatal deficiency of growth is effective and safe.

\section{EPO-149 Gonads and Gynaecology \\ Mayer Rokitansky syndrome cases reports} Liliana Mejia de Beldjenna'; Audrey Mary Matallana²;

Juan Pablo Rojas ${ }^{3}$; Adriana Martinez'; Mauricio Mejia ${ }^{4}$; Raul Astudillo ${ }^{4}$ ${ }^{1}$ Clinica Valle del LILI,Fundación Infantil Club NoeL,Universidad Libre,

Valle, Cali, Colombia; ${ }^{2}$ Universidad del Valle, Valle, Cali, Colombia; ${ }^{3}$ Universidad Libre, Valle, Cali, Colombia; ${ }^{4}$ Clinica Valle del LILI, Valle, Cali, Colombia

Background: Mayer Von Rokitansky Kuster Hauser (MRKH) syndrome is an anomaly of the female reproductive tract being the second cause of primary amenorrhea after gonadal dysgenesis. Incidence is $1 / 5000$ live Births. It is characterized by failure of the mullerian duct but normal secondary sex characteristics and Karyotype. There are two clinical forms: Type A consisting of absent $2 / 3$ superior vagina and uterus, symmetrical uterine remnants, normal tubes and ovaries. Type B with asymmetrical or absent uterine remnants, aplasia or hypoplasia of the tubes, cardiacs, renal $(40 \%)$ and skeletal anomalies $(5-10 \%)$. The syndrome is included in the differential diagnosis of primary amenorrhea. 
Objetive: Describe to patients with amenorrhea and Mayer Rokitansky Syndrome.

Methods: Cases from Pediatric Endocrine Clinic were Studied between 2008-2012.

Results: Five female patients between 9 and 15 years of age ( mean \pm SD $12,4 \pm 3,4)$ with amenorrhea and normal secondary sex characteristics were diagnosed as having MRKH;3 with type A anomalies. Kariotype was normal is all patients.

Conclusions: Anomalies of mullerian development presenting as primary amenorrhea should be suspected as MRKH. Diagnosis can be reached by proper physical exam and laboratory test done by a multidisciplinary team .

\section{EPO-150 Gonads and Gynaecology \\ A boy with Kallmann syndrome carring a microarrangement in Xp22.11: is there a connection? \\ Romana Marini'; Marco Cappa'; Carla Bizzarri'; \\ Maria Grazia Giuffrida'2 Lorenzo Sinibaldí2; Maria Cristina Digilio $^{3}$ \\ ${ }^{1}$ Bambino Gesù Children's Hospital, IRCCS, Endocrinology Unit, \\ Rome, Italy; ${ }^{2}$ Casa Sollievo della Sofferenza Hospital, IRCCS, S. \\ Giovanni Rotondo, Mendel Laboratory, Rome, Italy; ${ }^{3}$ Bambino Gesù \\ Children's Hospital, IRCCS, Genetics Unit, Rome, Italy}

Background: Kallmann syndrome (KS) is a genetic disorder characterized by the association of hypogonadotropic hypogonadism due to GnRH deficiency, and hypo/anosmia (with hypo/aplasia of the olfactory bulbs). Variable expression of phenotypic features associated with KS has been reported. Three modes of inheritance have been described in familial forms: X-linked recessive, autosomal dominant and more rarely, autosomal recessive. However, in an certain proportion of cases, transmission may be digenic or oligogenic. To date, five causative genes have been identified: KAL1(Xp22.3), responsible for the X-linked form, and FGFR1(8p12), FGF8(10q24), PROKR2(20p13), and PROK2(3p21.1), which are involved in the autosomal forms. Other genes involved in KS remain to be discovered as mutations in the genes identified so far are detected in less than $30 \%$ of patients.

Clinical report: We report a boy who, aged 3 years, was diagnosticated to have sensorineural bilateral hypoacusia and slight mental retardation. He was observed at the age of 14.5 years because of delayed puberty. On examination he presented: tall stature (0.6 SDS), overweigth (BMI 24), pubes stage PH2 according to Tanner and testicular volumes of $2 \mathrm{ml}$. He underwent to GnRH test and showed: LH peak of $1,1 \mathrm{mU} / \mathrm{ml}$ and FSH peak of $2,96 \mathrm{mU} / \mathrm{ml}$. The basal level of testosterone was $39,38 \mathrm{ng} / \mathrm{dl}$ and DHEAS was $650 \mathrm{ng} / \mathrm{ml}$. The test for olfactory acuity revealed anosmia. In the following four years GnRH test was repeated and the boy always presented an absent LH response and an extremely blunted FSH peak. Karyotype was normal but the CGH-array exam revealed a microarrangement of $180 \mathrm{~Kb}$ in Xp22.11.

Conclusions: SMS and PHEX map in this region. A mutation in the SMS gene was described in Snyder-Robinson mental retardation syndrome and defects in PHEX are a cause of X-linked hypophosphatemic rickets. We present a case of KS with mental retardation and hypoacusia associated to a triplication of Xp22.11. Further studies are needed to evaluated the possibility that the reported represents a novel genetic basis underlying KS.

\section{EPO-151 Gonads and Gynaecology \\ Three cases of pediatric patients with testicular microlithiasis showing gynecomastia and testicular enlargement \\ Chong Kun Cheon; Su Yung Kim}

Pusan National University Children's Hospital, Pediatrics, Yangsan si, Republic of Korea

Background: Testicular microlithiasis (TM) is a rare pathology characterized by localized or diffuse intratesticular foci of calcification. Its incidence in the pediatric population ranges from $1.1 \%$ to $4.2 \%$. The aetiology and the natural course of incidentally detected TM remain unclear.

Objective: To report three cases of TM in boys who complained of gynecomastia and bilateral testicular enlargement.

Results: Case 1) A 9.4-year-old boy presented with bilateral testicular enlargement accompanied by other pubertal signs. His bone age was 11 years and serum levels of LH and FSH after GnRH stimulation were within pubertal limits. Scrotal ultrasonography (US) showed TM in both testes. He revealed early puberty and no malignant evolution. Case 2) A 9.4-year-old boy with Down syndrome presented with bilateral testicular enlargement and accelerated growth velocity. His bone age was 11 years and serum levels of LH and FSH after GnRH stimulation were within pubertal limits. He had TM at US in both testes. He underwent right orchiopexy. He also revealed early puberty and no malignant change. Case 3) A 11-year-old boy complained of gynecomastia without other findings of puberty. His bone age was 11 years. He also had TM at US in both testes. No focal testicular lesion or malignancy developed during the review period.

Conclusions: Our report underline the usefulness of scrotal US for finding an occult TM in a patient with gynecomastia without other findings of puberty. In addition, TM may be predictive factor for early puberty evolution, but a large number of patients with a longer follow-up period may be needed to accurately discern the possible association between TM and precocious puberty.

\section{EPO-152 Gonads and Gynaecology \\ Bilateral galactocele in a male infant with Down syndrome and congenital hypothyroidism Ayla Güven; Suna Hancili \\ Göztepe Educational and Research Hospital, Pediatric Endocrinology, Istanbul, Turkey}

Introduction: Galactocele; is a rare disorder in infants and children, causing progressive painless enlargement of the breast. It is characterized by the accumulation of milk in a cystic formation in breast tissue with an unknown etiology

Case report: A sixth-month-old male, a known case of Down Syndrome (DS) and Congenital Hypothyroidism, who was euthroid with L-thyroxine, admitted to our hospital with the bilateral breast enlargement. There was no history of trauma, infection, maternal medication or drug use. Anthropometric measurements were normal by using growth charts for DS. Both breasts were $2 \times 2 \mathrm{~cm}$ in size and cyctic nature with palpation and not sensitive. There was no secretion from the nipples. Both testes were normal sized within the scrotum. CBC, liver and kidney function tests and LH, FSH, estradiol, total testosterone, cortisol, ACTH and thyroid hormone levels were normal while serum prolactin level was high $(42.5 \mathrm{ng} / \mathrm{ml}$, normal range 4.04 to $15.2 \mathrm{ng} / \mathrm{ml})$. The karyotype analysis was $47, \mathrm{XY}+21$ that performed previously. Sonography showed bilateral breast tissue with evident ductal structure and multiple anechoic microcysts. The cranial and pituitary magnetic resonance imagings were normal. Patient was followed up for one year. Since the complaints had been continuing, cyst aspiration was performed on the left breast. The histopathological examination of the specimen was amorphous and metachromatic material with benign ductal epithelial cells in a proteinaceous background, compatible with galactocele. Six months after the biopsy the patient's symptoms improved. Patient was followed for about 3 years and no recurrence was observed.

Conclusion: Galactocele should be considered in the differential diagnosis of infants presented with progressive enlargement of breast, characterized nontender and cystic mass. It is a usually unique clinical finding, but should be kept in mind that may be associated with some endocrinologic abnormalities.

\section{EPO-153 Gonads and Gynaecology \\ Normal testicular growth with normal AMH and inhibin-B but low testosterone values, indicative of isolated constitutive FSHR activation, in two patients with craniopharyngioma and multiple pituitary hormone deficiency (MPHD) Antonis Voutetakis ${ }^{1}$; Alexandra Magiakou ${ }^{2}$; Catherine Dacou-Voutetakis \\ ${ }^{1}$ Athens University, Pediatrics, Athens, Greece; ${ }^{2}$ Athens University, \\ Endocrinology, Athens, Greece}

Background: Normal testicular pubertal development is expressed by rising levels of testosterone, AMH and inhibin-B. In certain cases, however, genetic defects may lead to a discrepant function of Sertoli and Leydig cells.

Objective and hypotheses: To report a dichotomy between Leydig and Sertoli cell function in two patients with MPHD, resulting from craniopharyngioma.

Horm Res 2012;78(suppl 1) 289 
Methods: Patient 1, now aged 28yrs, was operated upon at the age of 5yrs for craniopharyngioma ("total" excision) and received no irradiation. He has been on substitution therapy for MPHD (rhGH, hydrocortisone, thyroxin and DDAVP) and reached a final height of $175 \mathrm{~cm}$. His testicular volume was $1-2 \mathrm{ml}$ up to age $13 \mathrm{yrs}$, at which time a progressive growth of the testes was observed up to $15 \mathrm{ml}$. Despite the increase in testicular volume, testosterone levels were $<100 \mathrm{ng} / \mathrm{dl}$ on various occasions (expected testosterone values: $250-600 \mathrm{ng} / \mathrm{dl}$ ).

Results: The peak LH and FSH values post GnRH at different ages were < 3 and $<2 \mathrm{mIU} / \mathrm{ml}$, respectively. The markers of Sertoli cell function, AMH and inhibin-B (determined by Elisa) were above the normal range (AMH $73 \mathrm{pmol} / \mathrm{L}$, repeat value $59 \mathrm{pmol} / \mathrm{L}, \mathrm{NV}: 14-38$ and inhibin-B $520 \mathrm{pg} / \mathrm{ml}, \mathrm{NV}<$ $300)$. Prolactin values had consistently been $<5 \mathrm{ng} / \mathrm{ml}$. Analogous evolution of testicular size and function was observed in patient \#2 (now aged 25yrs), who was operated upon for craniopharyngioma at age 10yrs and received irradiation postoperatively $(5000 \mathrm{R})$.

Conclusions: The data with regard to testicular function resemble those resulting from FSHR activating mutation. We suggest that the dichotomy between Sertoli and Leydig cell function in our patients could be the result of isolated constitutive activation of the FSHR of unknown pathogenetic mechanism(s).

\section{EPO-154 Gonads and Gynaecology \\ Hypergonadotropic hypogonadism in childhood fragile $X$ syndrome \\ Pelin Bilir'; Mehmet Emre Tapçilar ${ }^{2}$; Aypehan Akinci3; \\ Sevtap Hamdemir Kiliç ${ }^{4}$ \\ ${ }^{1}$ Ankara University, Medical Faculty, Department of Pediatric \\ Endocrinology, Ankara, Turkey; ${ }^{2}$ Gülhane Military Medical Faculty, \\ Department of Pediatric Endocrinology, Ankara, Turkey; ${ }^{3}$ nönü \\ University Medical Faculty, Department of Pediatric Endocrinology, \\ Malatya, Turkey; ${ }^{4}$ Private Practice, Obstetrics and Gynecology, Ankara, Turkey}

Background: The term "hypogonadism" refers to a group of disorders featuring complete or partial pubertal failure due to the hypothalamic-pituitarygonadal axis interruption at any level. Hypergonadotropic hypogonadism is a condition that associated with secretion of high levels of gonadotropins due to lack of target organ response. Fragile $\mathrm{X}$ syndrome is the most common inherited cause of mental retardation. It is caused by a CGG repeat expansion of three bases that occurs in the fragile X mental retardation 1 gene (FMR1) at Xq27.3. Classical physical features include large or prominent ears, a long face, high-arched palate, prognathism in older individuals, hyperextensible finger joints, subluxable thumbs, mitral valve prolapse, large testicles, premature ovarian failure, flat feet, and soft velvet-like skin.

Case: Fifteen years old girl was seen with primer amenorrhea at outpatient clinic. It was learned that she was started to evaluation at another Pediatric Endocrinology department with delayed puberty, social anxiety and intellectual disability. She has an elongated face, large and protruding ears and prepubertal. Parents were first degree cousin. Basaline LH:78.64 mIU/mL, FSH: $89.78 \mathrm{mIU} / \mathrm{mL}$, estradiol: $<20 \mathrm{pg} / \mathrm{ml}$. Overies were smaller than expected volume (left overy: $16 \times 7 \times 7 \mathrm{~mm}$, right ovary: $16 \times 8 \times 7 \mathrm{~mm}$ ) and there weren't any dominant follicule. Bone mineral density z score was -2 . Genetic analysis showed that she had Fragile X, FRAXA 21/28. Inorder to maintain breast development and prevent bone health, low dose estrogen therapy was started to patient. During follow-ups estrogen therapy dose was increased. At the end of two years her breast stage was 4. Pelvic sonography showed that uterus and ovarian size were increased too. Cyclic estrogen and progesterone therapy was started and she started to have regular menses.

Conclusion: During the differential diagnosis of delayed puberty and hypergonadotropic hypogonadism we wanted to remind the importance of genetic conseling for Fragile X syndrome.

\section{EPO-155 Gonads and Gynaecology}

Primary amenorrhea - Swyer syndrome

Paraskevi Zosi; Georgios Kafalidis; Dionisios Karakaidos; Zlatka Nikitaki; Thomais Vlachou; Likourgos Kanaris; Christos Karis General Hospital Nikaia- Piraeus “Agios Panteleimon”, Department of Pediatrics, Nikaia- Piraeus, Greece

Objective and hypotheses: Gonadal dysgenesis may be sporadic or familial. Stature is normal and none of the dysmorphic features of Turner Syndrome are present, although the gonads appears as streaks.

Methods: Two cases of gonadal dysgenesis XY, female type (GD.XY Swyer Syndrome in a 17 and 15 years old girls are described. Presented to ours Hospital in out patient endocrinological units with primary amenorrhea.

Results: We describe clinical laboratory genetic and ultrasound data in two patients with the syndrom : Case 1: Girl 17 years old of height $170 \mathrm{~cm}>75$ th percentile completely postpubertal pupil hair $(\mathrm{PH}) 2-3$, axially hair $(\mathrm{AH})$ 2, breast (B) 1-2. Puberty stating according to Tunner states: elevated LH and FSH (30 and $146 \mathrm{mu} / \mathrm{ml}$ ) and normal testosterone, E2 DEHAS $28 \mathrm{ng} / \mathrm{dl} 12$ $\mathrm{pg} / \mathrm{ml}$ and $240 \mathrm{mg} / \mathrm{dl}$. Karyotype 46XY chromosomal analysis and FISH with Subtelomeric XY Probes Results: 46XY ish X (XpYp -,XqYq-), Y (XpYp,XqYq-) In ultrasound a hypoplastic uterus, and small ovary with some follicles up to 2-3 mm especially right. Case 2: Girl 15 years old of height 168 $\mathrm{cm}>75$ th percentile and puberty staging to Tunner (Picture 1) PH 2-3, AH 2 , B 1. Pick laboratory data FSH: 104,1 mu/ml, LH : 61,7 mu/ml, DHEA : $296 \mathrm{~g} /$ $\mathrm{ml}$ and normal value E2 : $13 \mathrm{pg} / \mathrm{ml}$ testosterone $20 \mathrm{ng} / \mathrm{ml}$ chromosal analysis (46XY). Ultrasound show child uterus and streak ovary.

Conclusions: The presence of an XY Karyotype mandates gonadectomy. The incident of gonadoblastoma in $46 \mathrm{XY}$ gonadal dysgenesis is $33 \%$ and this fact clearly argues for Prophylactic gonadectomy.

\section{EPO-156 Gonads and Gynaecology \\ A surprising case of premature thelarche after an anti-fungal therapy}

Grazia Morandi; Rossella Gaudino; Claudia Piona; Paolo Cavarzere;

Elena Monti; Franco Antoniazzi

University of Verona, Department of Life and Reproduction, Verona, Italy

Introduction: Estrogen-like endocrine disrupting chemicals (EEDC) are exogenous chemicals that alter endocrine system functions by interfering with estrogens. Several of these substances have been studied but many are still to be discovered. Griseofulvin is a mycotoxic product of Penicillium spp., used for the treatment of dermatophytoses.

Case report: A 4,8 years old girl came to our Clinic for the development of a premature thelarche in the previous two weeks. As she was adopted her perinatal history was unknown, her height was M SDS and her weight -1/M SDS, and Tanner's stages of B2, P1, A0. The bone age was 5 years and 9 months according to the Greulich and Pyle Atlas. Laboratory findings showed normal thyroid function, HPRL ( $8 \mathrm{mcg} / \mathrm{L})$, and betaHCG marker $(0,10 \mathrm{mcg} / \mathrm{L})$; the Gonadotrophin-releasing hormone analogue (GnRHa) stimulation test revealed high levels of Estradiol, both basal and stimulated $(411,0 \mathrm{pmol} / \mathrm{L}$ and $275,9 \mathrm{pmol} / \mathrm{L}$ ) with pre-pubertal values of FSH and LH (FSH from 0.2 to $0.7 \mathrm{U} / \mathrm{L}$ and $\mathrm{LH}$ from 0.4 to $0.9 \mathrm{U} / \mathrm{L}$ ). Pelvic ultrasound showed a postpubertal morphology uterus, an hyperechogenic thick endometrium and some ovaric corpuscolated cysts. Better listening to her history we realised that she had been assuming for the last two weeks an antifungal drug, Griseofulvin to treat Tinea Capitis. We suggested her to stop the drug administration. After a month blood basal Estradiol levels diminished to $73,4 \mathrm{pmol} / \mathrm{L}$ and the ultrasound didn't show anymore ovaric cysts even if the uterus still showed postpubertal features.

Conclusion: Although we couldn't either measure the quantity of griseofulvin in the blood stream and either its activity on the reproductive system we showed a regression of the clinical, biological and echographic signs of precocious thelarche. We don't know if griseofulvin can behave as an EEDC but attention is required in its use. 


\section{EPO-157 Growth}

Study of longitudinal growth data of normal infants in Qatar: comparison with WHO and CDC growth standards

Rania Elalaily; Ashraf Soliman; Mohamed Eldabbagh;

Hany Khalafallah; Maryam Elali; Maryam Elalaily

Hamad Medical Center, Pediatrics, Doha, Qatar

Background: Growth data distribution of the WHO and CDC shows differences which are particularly important during infancy.

Objective and hypotheses: To compare linear growth of Qatari infants to those published by WHO and CDC.

Methods: Longitudinal growth data for normal full-term infants in Qatar (Q) ( 150 boys and 150 girls) at birth and the age of 2, 4, 6, 12 and 18 months were compared to growth standards published by the CDC and WHO.

Results: The mean length for age of Q girls was higher than those published by the $\mathrm{CDC}$ and $\mathrm{WHO}$ at 12 and 18 months of age.

At the age of 12 month 2/136 boys were underweight compared to the WHOS whereas $4 / 136$ boys were underweight compared to CDC standards. At the age of 18 months $4 / 132$ boys were underweight using WHOS but only 1 was underweight using the CDCS. $3 / 137$ girls were overweight compared to the WHOS and 2/137 were overweight compared to the CDCS.

At 18 months, one girl (4/134) was overweight using WHOS and 2 were overweight using CDCS. Five boys (3.7\%) were overweight compared to WHOS and $2(1.5 \%)$ were overweight compared to CDCS.

Using the CDCS for weight for length has detected more wasted infants $\{9.0 \%$ and $6.5 \%)$ compared to using WHOS $(6.27 \%$ and $6.0 \%)$ for males and females respectively.

Conclusions: When WHO and CDC standards are compared, more Q infants were identified as overweight when the WHOS were used. By contrast more infants were identified as wasted or underweight when the CDCS were used. It appears that the WHOS are preferable because they are based on a leaner breastfed reference and because overweight is likely to be a greater problem in Qatar in the future.

\section{EPO-158 Growth \\ Linear growth and neurodevelopmental outcome of children with congenital hypothyroidism (ch) detected by neonatal screening \\ Ashraf Soliman'; Azzam Said²; Ahmed Elawwa'; AmI Sabt'; \\ Wael Saleem ${ }^{1}$ \\ ${ }^{1}$ Hamad Medical Center, Pediatrics, Doha, Qatar; ${ }^{2}$ Hamad Medical \\ Center, Neonatolgy, Doha, Qatar}

Background: Different L thyroxine doses given to infants with $\mathrm{CH}$ were associated with different growth and developmental outcome during childhood. Objective and hypotheses: To assess linear growth and neurodevelopment in children with $\mathrm{CH}$ diagnosed through neonatal screening in Qatar.

Methods: We studied the longitudinal growth pattern and assessed the neurodevelopment of $45 \mathrm{CH}$ children (25 girls, 20 boys) diagnosed through the national screening program in Qatar, for 6 years or more to examine the effects of initial T4 dosage ( $50 \mathrm{ug} /$ day) with adjustment of T4 dose to maintain serum fT4 concentrations within the upper quartile of normal range and TSH $<4 \mathrm{mIU} / \mathrm{ml}$.

The birth size of newborns with $\mathrm{CH}$ diagnosed through the screening program before January 2003, was recorded and their growth in weight and stature was monitored every 3 months for at least 6 years of life. The IQ of children were assessed between 3 and 6 years of age using The Wechsler Preschool \& Primary Scale of Intelligence (WPPSI-III).

Results: Birth weight, length and head circumference of patients $(3.21+/$ $0.43 \mathrm{~kg}, 50.5+/-3.21$ and $34.1+/-1.5 \mathrm{~cm}$ respectively) did not differ than those for 10,560 normal newborns with normal thyroid function (3.19+/- 0.59 $\mathrm{kg}, 50.5+/-2.2 \mathrm{~cm}$ and $34.2+/-1.7$ ).

During the first year $\mathrm{CH}$ children growth $(25.8+/-2.8 \mathrm{~cm} /$ year $)$ was similar to those for normal infants $(25.5+/-0.75 \mathrm{~cm} /$ year $)$. During the first 6 years, stature growth was normal in all children with $\mathrm{CH}$ vs $\mathrm{CDC}$ data. The mean HtSDS of CH children showed adjustment (+/- 0.5 SD) towards their midparental height SDS (MPHtSDS) only during the second year of life. Children mean HtSDS was higher by an average of 0.4 SD between the 2 nd and 7 th year of life.

51st Annual Meeting of the ESPE
Conclusion: These data proved that effective screening and treatment completely assures normal neurodevelopment and linear growth in patients with CH. Data showed that their HTSDS slightly exceeds their MPHtSDS during childhood.

\section{EPO-159 Growth \\ Ease-of-use of the prefilled device Norditropin Nordiflex ${ }^{\circledR}$; a prospective multicentre study in pediatric patients \\ Maithé Tauber'; Delphine Jaquet ${ }^{2}$; Monique Jesuran-perelroizen ${ }^{3}$; Marc Petrus ${ }^{4}$; Anne-Marie Bertrand ${ }^{5}$; Régis Coutant 6 \\ ${ }^{1}$ Hopital des Enfannts, Endocrinologie pédiatrique, Toulouse, France; ${ }^{2}$ Novo Nordisk, Medical Affairs Biopharm, La défense, France; \\ ${ }^{3}$ Pédiatrie, Toulouse, France; ${ }^{4} \mathrm{CH}$ de Bigorre, Pédiatrie, Tarbes, France; ${ }^{5}$ Hopital saint Jacques, Pédiatrie, Besançon, France; ${ }^{6} \mathrm{Hôtel} \mathrm{Dieu,}$ \\ Pédiatrie, Angers, France}

Background: Long-term daily subcutaneous injections are required to maximize the therapeutic effect of Growth Hormone treatment (GHT) during childhood. Ease-of-use characteristics of GH devices are therefore critical to optimize compliance and subsequent efficacy.

Objectives: The primary objective was to assess the relative ease-of-use of Norditropin NordiFlex ${ }^{\circledR}$ comparing with the previous device. Secondary objectives included the assessment of overall easiness of Norditropin NordiFlex ${ }^{\circledR}$ according to parents, patients and health care professionals.

Population and methods: 103 patients, aged $\geq 6$ years $(11.7 \pm 2.94$ years, $58.3 \%$ male) receiving GHT for at least one year prior to inclusion in this multi-center, open-label study running in France. The relative ease-of-use of Norditropin NordiFlex ${ }^{\circledR}$ comparing with previous device was assessed, after 6 -week of treatment, using a quantitative scale ranging from 0 to 10 , with $0=$ far less simple, $5=$ equivalent, $10=$ far more simple.

Results: The mean duration of prior GHT was $4.2 \pm 2.8$ years. $90.3 \%$ of patients were using a device with prefilled cartridge and $9.7 \%$ a device requiring product reconstitution. After 6-week of treatment, Norditropin NordiFlex ${ }^{\circledR}$ was assessed as easier than previous device $(6.9 \pm 2.5, p<0.001) .64 .4 \%$ of patients preferred Norditropin NordiFlex ${ }^{\circledR}$ over their previous device. Of note, $100 \%$ of patients previously using devices requiring product reconstitution preferred Norditropin NordiFlex ${ }^{\circledR}$. Among physicians and nurses, $75 \%$ assessed Norditropin NordiFlex ${ }^{\circledR}$ training as very easy and $85 \%$ considered simplicity as an added value of the device. $28 \%$ considered that Norditropin NordiFlex ${ }^{\circledR}$ might improve compliance or motivation for GHT.

Conclusions: Norditropin NordiFlex ${ }^{\circledR}$ was assessed as easier to use and preferred by a majority of patients/parents in comparison to other devices, and it was seen by HCPs as very easy to train with added value of overall simplicity. Ease-of-use of Norditropin NordiFlex ${ }^{\circledR}$ may have positive impact on compliance during long-term GHT.

\section{EPO-160 Growth \\ Effect of Russell-Silver syndrome (RSS) on linear growth: lesson from an affected baby who is a part of a twin pair and response to GH therapy \\ Aml Sabt; Ashraf Soliman; Ahmed Elawwa}

Hamad Medical Center, Pediatrics, Doha, Qatar

Background: RSS is a growth disorder characterized by slow growth before and after birth. Babies with this condition have a low birth weight and often fail to grow and gain weight. The average adult height of males is $151.2 \mathrm{~cm}$ and that of females is $139.9 \mathrm{~cm}$.

Objective: To compare linear growth of twin A ( with RSS) with her normal twin (B).

Results: This girl (A) was born as a part of twin at 36 weeks of gestation. The girl (A) birth weight $=1.82 \mathrm{~kg}$, length $=40 \mathrm{~cm}$. Clinical examination revealed 5 th finger clinodactyly, triangular face and mild body asymmetry. Hypomethylation at IC1 on the paternal chromosome was detected. At the age of 2 years she has a length $=72$ and weight $=8.1 \mathrm{~kg}$, with lack of any catch-up growth, whereas her normal twin had complete catch-up. A growth hormone (GH) stimulation test using glucagon showed a peak $\mathrm{GH}$ response $=14.9 \mathrm{ug} / \mathrm{L}$, IGF-I $=93 \mathrm{ng} / \mathrm{ml}$, with normal thyroid function. A trial of human GH therapy $(0.05 \mathrm{mg} / \mathrm{kg} / \mathrm{day})$ for 6 months showed a significant catch-up growth $($ gain $=$ 
$1 \mathrm{SD})$ and weigh gain ( $1.5 \mathrm{~kg} / 6$ months).

Conclusions: This girl with RSS had complete failure of catch-up during the first 2 years of life versus complete catch-up of growth in her normal twin. Although she had normal GH response to provocation and IGF-I level, short term GH therapy appears to be useful for accelerating her linear growth.

Linear Growth of RSS versus normal twin (RSS started on GH at 24 months)

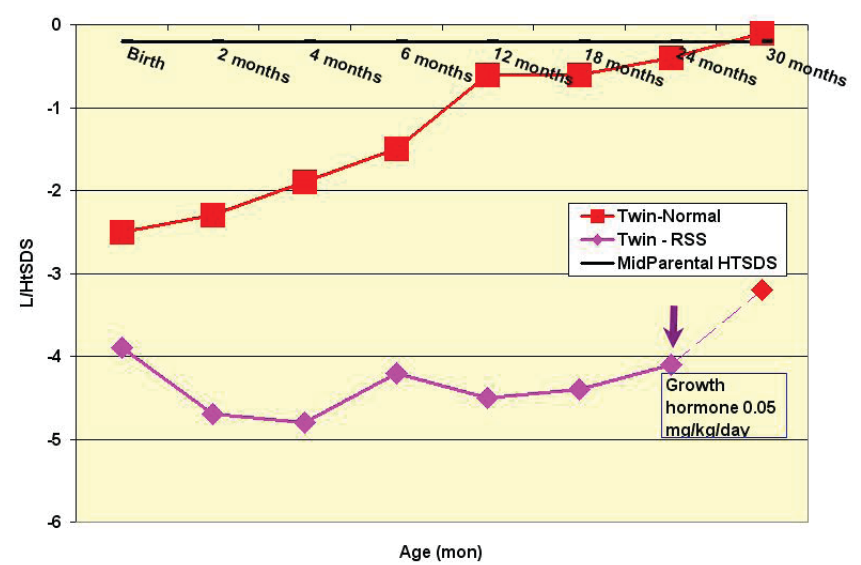

\section{EPO-161 Growth}

Morquio disease type IVA: clinical cases

Audrey Mary Matallana'; Liliana Mejia de Beldjenna;

Maria Alejandra Palma'; Jaime German Gonzalez'

'Universidad del Valle, Valle, Cali, Colombia; ${ }^{2}$ Clinica Valle del

LILI,Fundación Infantil Club Noel,Universidad Libre, Valle, Cali,

Colombia; ${ }^{3}$ Fundacion clinica Infantil Club Noel Universidad Libre,

Valle, Cali, Colombia; ${ }^{4}$ Centro Medico Imbanaco, Valle, Cali, Colombia

Background: Morquio disease was described by the Uruguayan pediatrician Luis Morquio.It is a congenital disease caused by a deficiency of the enzyme N ACETILGALACTOSAMINE 6 SULFATASE(MPS IV A) or B GALACTOSIDASE (MPS IV B ) and it frequency is $1 / 100.000$ live bitrths Accumulation of mucopolysacharides in tissues results in short stature, skeletal anomalies (vertebral column deformities), loss of hearing, visual anomalies (corneal opacities), cardiac, hepatic and respiratory problems life expectancy is 40 years.

Objetives: To describe four patients with Morquio's disease type IVA.

Methods: Description of physical findings, radiologic and laboratory finding in patients with short stature and Morquios disease.

Results: Four patients :3 male, 1 female mean age 2 years ,consulting for short stature ( $>3$ SDFOR AGE) deformities age 18 months, dolichocefhaly, serrated teeth, thoracic kyphosis, pectum carinatun, globular abdomen, joint thickening and genu valgus and one with corneal opacities. Leucocytes enzymatic activity of galactose 6 sulfate with decreased (Morquio type IVA.)

Conclusion: Patient as young of 18 months old with short stature and skeletal deformities it should be suspected Mucopolysacharidosis. Since other tissue involment appear later on, a cardiology and ophthalmology evaluation should be done including a bone marrow analysis. Theres is promising results with new enzymatic replacement therapy.

\section{EP0-162 Growth \\ Impaired growth in a patient with Caroli syndrome \\ Dick Mul' ; Danielle M Hendriks ${ }^{2}$ \\ ${ }^{1}$ Haga Ziekenhuis / Juliana Children's Hospital, Pediatric endocrinology, The Hague, Netherlands; ' Haga Ziekenhuis / Juliana Children's Hospital, Pediatric gastroenterology, The Hague, Netherlands}

Background: Caroli syndrome (CS) is a syndrome characterized by saccular, intrahepatic bile duct dilatation associated with congenital hepatic fibrosis Caroli's disease is a hepatic manifestation of autosomal recessive polycystic kidney disease (ARPKD) and fibrocystin/polyductin is the PKHD1 gene product responsible for ARPKD. The pathogenesis of CS is not completely understood and until now there are no reports on growth disorders in CSpatients with normal liver function.

Objective: Demonstrate a case of CS with impaired growth.

Case report: This patient was initially diagnosed with congenital hypothyroidism (CHT), with a homozygous mutation in the TPO gene. Growth was in the target height until age 4 . Then gradual growt retardation was shown, and in the same periode liverenlargement was detected, leading to the diagnosis of CS. Endocrine workup revealed Insulin like Growth Factor (IGF)-1 values below limits of detection, while GH stimulation test showed low normal GH levels. Thyroxin dosage has to be high to maintain biochemical euthyroidism. The latter was, after evaluation, suspected to be due to increased deiodinase activity in the liver. Bone age was slighly retarded. Actual liver function is normal, and no protein loss by stool or urine is present.

Conclusions: This is the first case of longterm follow up of growth in a child with Caroli syndrome. A previous single case showed a patient with the same combination of CS and CHT, but without growth data of longer duration. Underlying endocrine mechanisms in this case, leading to growth retardation might include changes in the metabolism of both IGF-1 and thyroid hormone. Hypothyroidism was corrected by thyroxin supplementation, and growth promotion could be considered to be provided by either growth hormone or recombinant IGF1 treatment. Further research on the specific role of liver disease on the peripheral actions of hormones is warranted.

\section{EPO-163 Growth \\ HRD syndrome with hyperthyroidism two family cases reported}

Sarra Barrak; Yosra Arfaoui; Hager Barakizou; Souha Gannouni;

Fethi Bayoudh

Military hospital, Paediatrics, Tunis, Tunisia

Background: HRD syndrome (Hypoparathyroidism, mental and growth Retardation Dysmorphism ) is a congenital idiopathic hypoparathyroidism; characterized by severe pre-natal and post-natal growth retardation as well as dysmorphic features, seizures and susceptibility to repeated life-threatening infections. It is a rare autosomal recessive disorder. mutation of TBCE gene is localized in the chromosome1q42-43.

Objective: We reported this syndrome in a Tunisian boy and his sister who were given birth after a consanguineous marriage.

Methods: They also exhibit subclinical hyperthyroidism for the boy and basedoew disease for the daughter At the age of 2 months, the boy presented hypocalcaemia convulsions. He had features facial abnormalities : deep- set eyes, depressed nasal bridge and peaked nose, large philtrum, micrognathia and retrognathia, thin lips, ear abnormalities, microcephaly ,small hands and feet and growth retardation with SDscoring -3 for height, weight and head circumference.

Results: Laboratory results showed Hypoparathyroidism. He was supplied with 1 alpha hydroxycholecalciferol and calcium supplementation .However, there was no changes in his growth pattern.His sister, however, had Hypocalcaemia at birth. She presented the same clinical picture. At the age of 2years and six months and she has a basedoew disease. The clinical symptoms at diagnosis were hypoglycemia goiter, and tachycardia. Graves' disease is confirmed, by elevated blood levels thyroid hormones (T4: 3 times the normal) and a suppressed TSH, thyroid antibodies are high (ATPO : 30 times the normal) . she was treated with neomercazol and propranolol but there was no response to this treatment. A cytogenetic study was made for the two patients It showed a mutation of TBCE gene localized in the chromosome 1q 42-43, which was homozygous for both children and heterozygous for both parents. The boy died at the age of 9 years and his sister at the age of 3 years $1 / 2$. Conclusion: Prenatal diagnosis with cytogenetic study is a good way to prevent the disease. 


\section{EPO-164 Growth}

Can medical school examination serve as a screening method for growth anomalies in children?

Alexandra Goischke ${ }^{\text {; }}$ Camille Garcin²; Caroline Elie3; Geneviève Richard ${ }^{4}$; Anne Thery ${ }^{4}$; Michel Polak ${ }^{5}$

${ }^{1}$ Hôpital Necker Enfants-Malades, AP-HP, Université Paris Descartes, Paediatric Endocrinology, Gynecology and Diabetology, Paris, France; ${ }^{2}$ Hôpital Necker Enfants-Malades, AP-HP, Department of Biostatistics, Paris, France; ' ${ }^{3}$ ôpital Necker Enfants-Malades, AP-HP, Université Paris Descartes, Department of Biostatistics, Paris, France; ${ }^{4}$ DASES School health department, Paris, France; ${ }^{5}$ Hôpital Necker EnfantsMalades, AP-HP, Université Paris Descartes, CRMERC, Paediatric Endocrinology, Gynecology and Diabetology, Paris, France

Background: Growth monitoring in children is an important tool to detect health problems. However growth screening is still poorly implemented in France, especially in comparison to some other European countries.

Objective and hypotheses: The aims of our study was to determine if systematic school examination is a valid screening model to detect growth anomalies, to review the frequency of various causes in a representative sample of Paris school children and to identify those who need a more specialized follow-up.

Methods: Stratified sampling has been used to select 75 schools of Paris city (out of 345 regrouping 17000 school children aged 5 to 11 years). Two systematic health visits, organized by the school medical department (around 5 and 9 years) served to collect auxological parameters retrospectively in an anonymous way.

Results: 2891 children were screened and 2736 have been included, as for 155 , no auxological parameters were available. Complete information for the two school visits was acquired for only 1698 children. 12 children presented short stature below -2SD, no follow-up information were available for 9 of them for the school medicine system. Anomalies in height velocity were detected for 198 children, a follow up was known for 10 children. Height and weight were constantly higher in our sample than those of the Sempe reference data used in France (mean + 0.9 SD for height).

Conclusions: Systematic school health visits offer a good opportunity to screen the whole population. However, the best method to do so remains to be find in France. Further work is in progress by the school health department to unravel the causes of growth anomalies detected in our survey. Growth curves used as reference in France are no longer accurate. Further studies are necessary to establish a new standard. Supported by an educational ground from Novo Nordisk.

\section{EPO-165 Growth}

\section{Usefulness of GHRH stimulation test in diagnostic work-up of growth hormone deficiency in children}

Monika Obara-Moszynska; Marek Niedziela

Poznan University of Medical Sciences, Department of Pediatric

Endocrinology and Rheumatology, Poznan, Poland

Background: Pharmacological stimulation tests used for over 30 years to evaluate the pituitary reserve of growth hormone $(\mathrm{GH})$ are crucial for the diagnosis of growth hormone deficiency (GHD). One of them is a test with the biosynthetic growth hormone releasing hormone (GHRH). It provides the potential information to differentiate patients with pituitary dysfunction with patients with hypothalamic abnormalities. However, using this test as a routine in GHD diagnostic work-up is discussed. The aim of the study was to assess the usefulness of the GHRH test in diagnosis of GHD.

Methods: The study group consisted of 20 children with short stature and a prior documentation of GHD in a screening test (GH secretion during sleep). All children were prepubertal, aged 4-16 years, with normal BMI similar to age ranges. In all children two or three standardized stimulation tests were performed and one of them was GHRH test.

Results: Mean peak concentration of GH in the GHRH test was $14.6 \mathrm{ng} / \mathrm{ml} \pm$ 11.53. GHRH test correlated with other pharmacological tests in 9 children. In 8 children MRI showed pituitary hypoplasia, in 1 patient the pituitary hypoplasia and pituitary stalk agenesis and in 1 septo-optic dysplasia. All patients with pituitary malformations except one with hypoplastic pituitary gland presented GH levels $<10 \mathrm{ng} / \mathrm{ml}$ in GHRH test. The sensitivity of the GHRH test in the study group was $45 \%$.
Conclusions: The obtained results allow to conclude that a high correlation between GHRH test and anatomical changes in the pituitary gland provides a high predictive value of this test. In individual clinical cases the knowledge about the level of impairment within the hypothalamic-pituitary area can determine the diagnostic and therapeutic procedures. On the other hand it should be emphasized that the normal GH concentration in GHRH test does not exclude pituitary insufficiency due to a frequently occurring in children hypothalamic etiology of GH secretion disorder.

\section{EPO-166 Growth \\ The effect of clonidine therapy on height velocity of children with constitutional growth delay Zahra Razavi \\ Hamedan University of Medical Sciences, Ped - Endocrinnology, Hamedan, Islamic Republic of Iran}

Background: Constitutional delay of growth and puberty (CDGP) is the most common presenting form of short stature which is seen more in boys than in girls. This variant of normal growth, can be associated with significant psychological stress warranting treatment in some affected individuals.

Context: It has been shown that alpha 2-adrenoceptor activation by clonidine have a stimulatory effect on GH release. However, effect of clonidine therapy to promote growth rate in children with CDGP is controversial.

Objective: To assess the effects of clonidine on short-term growth rate in children and adolescents with constitutional growth delay.

Material and methods: A prospective, randomized, case controlled clinical study was conducted in 48 prepubertal boys (age $6-12$ years) with CDGP(height standard deviation score, $-2.54 \pm 0.63 \mathrm{~cm}$; bone age delay 2.1 $\pm 0.2 \mathrm{~cm}$ years; ) at an outpatient endocrine clinical. Subjects were randomized ( $n=24$ /group) to 6 months observation followed by additional nightly dose of clonidine $(0.1 \mathrm{mg} / \mathrm{m} 2$ by mouth) in trial group for another 6 months. Main outcome measure was growth rate 6 months before and 6 months after treatment with clonidine. The results of 6 months of clonidine therapy were compared with those of 6 months of observation without any treatment.

Results: Mean age of treated group was 10.92 years(range 6-12 years) and mean age of control group was 10.21 years (range 6-12 years). Addition of 6 months clonidine resulted increase growth rate $(1.45, \mathrm{~b} 0.129 \mathrm{~cm}$ vs $0.90,, \mathrm{~b} 0.50$ $\mathrm{cm})$ but difference was not significant between the two groups $(\mathrm{p}=0.29)$. Growth velocity $(2.9+/-0.91 \mathrm{~cm} / 6)$ month in baseline was not improved by clonidine $(3.1+/-0.98 \mathrm{~cm} / 6 \mathrm{month})$ compared with the control group control (before study: $2.9+/-0.94 \mathrm{~cm} / 6$ months, after study2.4+/-0.47 6 months). Conclusion: Clonidine therapy resulted in no significant difference in height standard deviation score and growth velocity of children with constitutional delay of growth \& puberty.

\section{EPO-167 Growth \\ Vitamin D status in children with growth hormone therapy}

Adela Chirita Emandi'; Ioana Micle' ${ }^{1}$; Monica Marazan²; Corina Pienar 'Emergency Hospital for Children "Louis Turcanu", Endocrinology,

Timisoara, Romania; ${ }^{2}$ University of Medicine and Pharmacy, Pediatrics, Timisoara, Romania

Background: Recent studies have suggested that vitamin D deficiency in children is widespread. The Endocrine Society's Clinical Practice Guideline, published in July 2011 recommends screening of 25-hydroxyvitamin D $[25(\mathrm{OH}) \mathrm{D}]$ in groups of people at high risk for vitamin D deficiency. However, vitamin D status evaluation in children with growth hormone $(\mathrm{GH})$ replacement therapy is not one of the recommendations.

Objective: To survey the serum levels of $25(\mathrm{OH}) \mathrm{D}$ in a sample of children with growth hormone replacement therapy and search for possible correlations between serum levels of vitamin D and parameters that can influence it. Population and method: 10 patients under treatment with $\mathrm{GH}$ for 1 up to 4 years, with age from 6 to 13 years, were selected. We calculated Pearson correlations between $25(\mathrm{OH}) \mathrm{D}$ levels and parameters like: age above or below 9 years (pubertal onset age); growth velocity $(\mathrm{cm})$ in the last year, standard deviation score(SDS), standard deviation score difference in the last year, bone age delay, IGF1 and GH dose; that were assessed during follow-up visits. Vitamin D was measured in winter months (January-February). 
Results: All patients had vitamin D serum levels below normal. $70 \%$ of children were vitamin $\mathrm{D}$ insufficient $(25(\mathrm{OH}) \mathrm{D}<20 \mathrm{ng} / \mathrm{ml})$, while $30 \%$ were vitamin D deficient $(25(\mathrm{OH}) \mathrm{D}$ of $21-29 \mathrm{ng} / \mathrm{ml})$. We found relevant correlations between vitamin $\mathrm{D}$ and bone age delay $(\mathrm{r}=-0.718, \mathrm{p}=0.02)$; SDS $(\mathrm{r}=0.617$, $\mathrm{p}=0.05)$; and age $>9$ years $(\mathrm{p}=0.453, \mathrm{p}=0.02)$. All other correlations did not reach statistical significance.

Conclusion: Our small study is signaling that vitamin D deficit could be highly prevalent in children receiving GH therapy, underlying the necessity for vitamin D screening in this group of patients and also vitamin D supplementation. Larger studies are needed to prove these findings with more statistical power.

\section{EPO-168 Growth \\ Growth deficiency in Cornelia de Lange syndrome - case report}

Ilinka-Andreea Grozavu' ; Andra-Iulia Loghin²; Adrian Condrea²; Corina Galesanu

'University of Medicine and Pharmacy "Gr. T. Popa", Endocrinology, lasi, Romania; ' University of Medicine and Pharmacy, Endocrinology, IASI, Romania

Background: Cornelia de Lange syndrome (CdLS) is a rare developmental disorder, characterized by a distinctive facial appearance, prenatal and postnatal growth deficiency, psychomotor delay, behavioral problems, and malformations of the upper extremities. Failure to thrive may be caused by gastroesophageal reflux and other issues with feeding, growth hormone deficiency or resistance.

Objective and hypotheses: To describe a rare cause of short stature, to make a correct differential diagnosis and to find out a good management of the treatment.

Methods: We present a 37 year old female affected by the syndrome in question, with the principal clinical characteristics (short stature, facial criteria, structural anomalies of the limbs). She was a second child of nonconsanguineous marriage, born at 36 weeks, normal delivery with a birth weight of $2850 \mathrm{~g}$, height of $48 \mathrm{~cm}$.

In her early childhood, she was repeatedly hospitalized in the pediatric service because of feeding problems and growth delay. The diagnosis was established at 14 years, after other syndromes were excluded.

The patient's general state of health was acceptable, without cardiac, respiratory or renal alterations. She came to us with severe back pain, weight gain and sleepiness. Clinical and paraclinical examinations revealed height of $137 \mathrm{~cm}(-4,5$ DS and -2 DS compared with her growth prognosis), normal menses, pituitary hormones in normal ranges, dyslipidemia. Bone densitometry (DXA scan) revealed osteoporosis( Z-score spinal=-3,8 and whole body=$1,4)$. She started bisphosphonate treatment ( risedronate $35 \mathrm{mg}$ once a week) with reevaluation after 1 year.

Results: An interdisciplinary approach to therapy and treatment of any medical issues that arise in CdLS is recommended. Mildly affected individuals may have no major malformations and near normal intelligence, and are able to live independently and reproduce.

Conclusions: We think about the benefits of growth hormone therapy in children with CdLS regarding the improving height and bone mass acquisition.

\section{EPO-169 Growth \\ Serum zinc levels in HIV sero-positive children attending university of Port Harcourt teaching hospital, Nigeria \\ Iroro Yarhere; Rosemary Ugwu; Augusta Eneh \\ UPTH, Paediatrics, Port Harcourt, Nigeria}

Introduction: Zinc is a micronutrient necessary for various enzyme activities, growth, mucosal epitheliazation and support of a healthy immune system. Serum zinc concentration declines during acute and chronic infections including HIV. Serum zinc is reported to be below normal range in adults with HIV, but there are few studies in children with HIV in Nigeria.

Aim: The aim of the study was to determine the serum zinc levels in HIV sero-positive children in the University of Port Harcourt Teaching Hospital and to compare this with age and sex matched controls.

Method: A case-control study of 70 HIV sero-positive children (subjects) and 70 HIV sero-negative children (controls) carried out between 1st of June
2009 and 31st of May 2010 in UPTH. Anthropometry, clinical and immunologic stages of HIV positive children were determined and serum zinc levels of subjects and controls were measured, using FAS. Arithmetic means were compared using Student $t$ test. Chi square test were used to compare proportions, $\mathrm{p}$ value $<0.05$ was considered significant.

Results: 71 children had serum zinc levels below $80 \mu \mathrm{g} / \mathrm{dL}$ giving a prevalence of zinc deficiency of $50.7 \%$.

Forty two $(60 \%)$ of the subjects were zinc deficient as against $29(41.4 \%)$ of the controls. The mean serum zinc level in subjects was significantly lower than that of controls $(65.8 \pm 33.3 \mu \mathrm{g} / \mathrm{dL}$ versus $86.1 \pm 23.8 \mu \mathrm{g} / \mathrm{dL}, \mathrm{p}=0.000)$. Among the subjects, the mean serum zinc level was lowest in children $18-59$ months, $(p=0.147)$, in those with HIV stages 3 and $4(p<0.001)$ and in those with severe underweight $(\mathrm{p}=0.003)$ and stunting $(\mathrm{p}=0.014)$.

Conclusion and recommendation: A high prevalence of zinc deficiency was recorded in the study.

Zinc supplementation should be routine in HIV children Age grp Subjects Controls n zinc ug/dL n zinc ug/dL t p 18 - 594761.54786 .3 -4.4 0.00160 $1192072.92084 .3-1.30 .199120-192365.3396 .7-0.560 .0607$

\section{EPO-170 Growth}

\section{Final height in congenital adrenal hyperplasia due to 21-hydroxylase deficiency: the Egyptian experience}

Abeer Atef Alashmawy ${ }^{1}$; Nermin Salah Eldin ${ }^{1}$; Abeer Hamed ${ }^{1}$; Nora Badawi'; Tarek Fareed ${ }^{2}$

${ }^{1}$ Kasr Alaini- Faculty of medicine, Cairo University, Diabetes, Endocrine and Metabolism Pediatric Unit(DEMPU), Cairo, Egypt; ${ }^{2}$ National

Research Center, Pediatrics Department, Cairo, Egypt

Background: Congenital adrenal hyperplasia due to 21-hydroxylase deficiency (21-OHD CAH), accounts for over $95 \%$ of diagnosed cases of CAH. One of the primary aims (21OHD) therapy is to guarantee a normal pre-pubertal and pubertal growth, to reach a final height (FH).

Objective and hypotheses: To evaluate the factors that may influence the growth and $\mathrm{FH}$.

Methods: A retrospective review was conducted for 36 patients with 21-hydroxylase deficiency (26 females and 10 males) (18 salt wasters, SW, and 18 simple virilizing, SV) who were followed up from the age of their first diagnosis until they reached FH (from: 1980 to 2008). The impact of gender, time of diagnosis, salt wasting manifestations and treatment regimen on $\mathrm{FH}$ were studied.

Results: Mean Corrected FH was $-2.26 \pm 0.41$. Mean FH SDS was -3.26 , mean FH in males and females: 153.29 and $144.81 \mathrm{~cm}$ respectively. Eleven patients received leutinizing hormone releasing hormone analogue as they had precocious puberty at mean age of $5.7 \pm 1.9$ years in males and $6.1 \pm 1.3$ years in females. Height gain during puberty was $24.5 \pm 13.4 \mathrm{~cm}$ in the treated and $15.6 \pm 6.1 \mathrm{~cm}$ in the untreated cases $(\mathrm{p}=0.03)$.

There was no significant difference between SV and SW cases as regards FH SDS in both males and females or chronological age at FH. Male patients with SW manifestations had entered puberty the earliest when compared to other groups and had the most compromised FH (SDS) outcome. There was a positive correlation between FH (SDS) and height gained during puberty $(\mathrm{r}=0.48$, 2 -tailed $p=0.012$ ). There was a significant negative correlation between the dose of hydrocortisone in the first year $(30.1 \pm 7.2 \mathrm{mg} / \mathrm{m} 2 /$ day $)$ and both $\mathrm{FH}$ $(\mathrm{SDS})(\mathrm{r}=-0.47, \mathrm{p}=0.005)$ and mean growth velocity in first year of life $((\mathrm{r}$ $=-0.472, \mathrm{p}=0.004)$.

Conclusions: Under our conditions of management, $\mathrm{FH}$ was compromised compared to the genetic potential .Early diagnosis and good compliance would appear to improve the outcome. The doses of hydrocortisone in the first year of life and height gain during puberty were related to FH. 


\section{EPO-171 Growth}

The features of intracardiac hemodynamics in children with growth delay

Maria Petrova ${ }^{1}$; Andrey Averyanov'; Nina Bolotova ${ }^{2}$

${ }^{1}$ Saratov State Medical University, Clinical hospital SGMU, KPDB,

Saratov, Russian Federation; ${ }^{2}$ Saratov State Medical University,

Department of Propedeutics of Pediatric diseases, Pediatric

Endocrinology and Diabetology, Saratov, Russian Federation

The aim: To study the intracardial hemodynamic in children with growth delay of different origin.

Methods: 44 patients were examined: 1 st group - 8-15 y.o., with growth hormone deficiency $(\mathrm{n}=15$, age $13,3+2,6$, mean body surface area is $0.92 \mathrm{~m} 2$ ) and constitutional growth delay $(\mathrm{n}=13$, age $7,8+3,1$, mean body surface area is $0.92 \mathrm{~m} 2$ ). Control group - 16 healthy children $7,8+3,1$ y.o. adaptedly sex and body surface area $(0.93 \mathrm{~m} 2)$, which is essential for evaluating echocardiographic parameters. History, general examination, echocardiography were evaluated.

Results and conclusions: $40 \%$ patients of the 1 st group had 3 and more inborn anomalies of heart - more than in population of healthy children. In the 2nd group the authentic increase of contractile function of left ventricular was revealed: decrease of end-systolic volume, end-diastolic volume index and increase of ejection fraction. In both groups the mean cardiac index is lower than in control group.

\section{EPO-172 Growth}

\section{Nutritional supplementation accelerates growth of short children: comparison to growth hormone therapy}

Zvi Zadik'; Tali Sinai'; Ram Reifen'; Amnon Zung ${ }^{1}$

${ }^{1}$ Kaplan Medical Center, Pediatric Endocrinology, Rehovot, Israel;

${ }^{2}$ Hebrew University, Nutritional Sciences, Rehovot, Israel

Background: Nutritional status modulates growth, in part, by altering the $\mathrm{GH}$-axis. In previous studies we found a positive correlation between caloric intake and growth velocity in normal short children. Similar correlations were also found with IGF-I, GHBP and IGFBP3 levels.

Objective and hypotheses: To assess the effect of nutritional supplementation on growth of short children.

Methods: Following a 6 month observation participants were randomly allocated to growth hormone therapy, nutritional program or passive observation. Nutritional program included iron, zinc, vitamin A, trace elements and energy per DRI for 6 months and an individually fitted program for additional 6 months. 3 monthly: height, weight, dietary intake and serum IGF1 were collected.

Results: Pre study intake analysis as DRI\%, revealed partial deficiency of energy, vitamin $\mathrm{A}$, vitamin $\mathrm{D}$, folic ac, iron, Zinc, Mg, Mn. In $25 \%$ of the patients high protein intake was found. In the first 6 months growth rate of nutritional supplemented and growth hormone treated were similar, but significantly greater than noted in the observation group $7.9 \pm 1.8$ vs. $4.6 \pm 1.3 \mathrm{~cm} /$ $\mathrm{yr}, \mathrm{P}<0.001$. Observation group of the AGA children did not differ from the SGA observation group. Comparison of the GH treated to the nutrition supplemented group demonstrated a growth rate of $8.4 \pm 1.8$ vs. $6.7 \pm 1.4 \mathrm{~cm} / \mathrm{yr}$., $\mathrm{P}<0.01$. By multiple linear regression patients with relative excess of protein/ carbohydrate load as DRI\% grew less the those with a balanced protein/ carbohydrate load. IGF1 was positively correlated with energy intake and growth rate 0.45 and 0.54 respectively $p<0.01$. Patients on the individually fitted program grew better than the others.

Conclusions: 6 months of vitamin A, iron and zinc supplementation induces growth acceleration in short children born SGA with subnormal nutrients intake that is as efficacious as growth hormone therapy. A substantial number of Idiopathic short children suffer from subnormal nutrition.
EP0-173 Growth

Experience of using domestic growth hormone Galina Chistousova

Territorial Children's Clinical hospital, Territorial Children's Clinical hospital, Perm, Russian Federation

Background: The clinical effectiveness of growth hormone with long-term use.

Objective and hypotheses: Evaluating clinical efficiency of Rastan somatotropin preparation (Rastan, formation Pharmstandard, Russia) in children with growth hormone deficiency (GH)

Methods: 15 patients with proven GH deficiency under continuous rGH therapy for 3 years were examined. The diagnosis was based on $2 \mathrm{GH}$ stimulation tests (maximum release: $3.14 \pm 0.48 \mathrm{ng} / \mathrm{ml}$ in clonidine test, $3.37 \pm 0.58 \mathrm{ng} / \mathrm{ml}$ in insulin test). Isolated GH deficiency was $93 \%$ in 14 persons, hypothyroidism was detected in 1 person with $6.6 \%$. In the course of observing the children, bone age, growth SDS, BMI SDS were evaluated. The bone age was $7.85 \pm 0.81$ years, the retardation 3.65 years. The growth SDS was $-3.1 \pm 0.2$, BMI SDS $-0.65 \pm 0.47$. The drug under test was administered on daily basis, subcutaneously, in the evening before going to bed. The drug was administered at $0.033 \mathrm{mg} / \mathrm{kg}$ of body weight.

Results: In the first year of treatment, a considerable average body height gain of $9.28 \pm 0.5 \mathrm{~cm}$ was observed in the children. Correspondingly, the growth retardation decreased, the growth SDS was $-2.46 \pm 0.19(\mathrm{p}=0.028)$, BMI SDS values remaining at $-0.62 \pm 0.44$. The ossification rate showed no considerable increase, the bone age was $8.63 \pm 0.82$ years only. The chronological retardation was 3.9 years. During the 3 rd year of treatment, the gain height was $5.22 \pm 0.51 \mathrm{~cm}$ increased the growth SDS values in these children considerably: $-1.82 \pm 0.17$. With respect to the 2 nd year of therapy, we noted a significant improvement of the growth SDS $(p=0.042)$, BMI SDS of $-0.11 \pm 0.4$. We also noted an increase in ossification rate of $9.25 \pm 0.63$ years. The chronological retardation was decreased to 3.5 years.

Conclusions:These clinical impressions demonstrate sufficient therapeutic effect of Rastan for treating growth hormone deficiency in children.

\section{EPO-174 Growth}

\section{Long-term experience of growth hormone administration in children with somatotropic insufficiency}

Galina Galkina'; Irina Cherniak2 ; E Kleshchenko²; Aleksandr Afonin ${ }^{3}$. V Shashe/'; Svetlana Rulik

'Rostov Scientific Research Institute of Obstetrics and Pediatrics, Ministry of Health Care and Social Development, Pediatric Endocrinology, Rostov on Don, Russian Federation; ${ }^{2}$ State Budget Educational Institution of Public Health, "Krasnodar Regional Children's Clinical Hospital", Pediatric Endocrinology, Krasnodar, Russian

Federation; ${ }^{3}$ State Budgetary Institution "Rostov Scientific Research Institute of Obstetrics and Pediatrics", Ministry of Health Care and Soc, Pediatric Endocrinology, Rostov on Don, Russian Federation; ${ }^{4}$ State Budget Educational Institution of Higher Vocational Education "Kuban State Medical University", Pediatric Endocrinology, Krasnodar, Russian Federation

Background: Efficiency of administering recombinant growth hormone $(\mathrm{rGH})$ preparations in children with somatortropic insufficiency (SI) is beyond any doubt, however, the necessity of long-term prospective follow up of such patients still remains topical.

Objective: Studying dynamics of physical development parameters in children with SI of southern Russia (Rostov Region and Krasnodar Region) against the background of 10 years of $\mathrm{rGH}$ treatment.

Methods: 90 patients with SI were observed originally [61 (67.8\%) boys and $29(32.2 \%)$ girls $)$ aged from 4 to 15 , with growth inhibition from -4.6 to $-2.5 \mathrm{SDS}$, with bone age delay from chronological by $4.6 \pm 2.6$ years. GH deficiency was confirmed by performing classical stimulation tests. Reasons of GH deficiency were verified by magnetic resonance imaging. In $50 \%$ of patients isolated SI was revealed, in $50 \%$ - hypopituitarism. All children received $\mathrm{rGH}$ in dosage $0.033 \mathrm{mg} / \mathrm{kg}$ daily, s/c, in the evening.

Results: On the basis of 10-year experience it was found out that the maximum growth effect was revealed in children who started treatment under the age of 10 . Growth rate in children with isolated SI was more than in children with hypopituitarism. The analysis of administration efficiency of rGH preparations ("Norditropin Nordilet", "Humatrop", "Saizen", "Genotropin") from 
2006 to 2008 (Group 1) revealed the average growth gain $-8.8 \pm 2.3 \mathrm{~cm}$ a year. From 2009 to 2011 (Group 2) children received rGH treatment with preparation "Rastan" (Russia). Growth rate made from $8.7 \pm 2.9 \mathrm{~cm}$ a year. Statistically no significant distinctions between groups were detected, and that evidences for equivalence of therapeutic effect of various GH preparations. Conclusions: Long prospective follow up of children with GH deficiency manifested a good growth stimulating effect of somatortropin. The earlier children with GH deficiency started replacement therapy with rGH and the longer it lasted, the quicker children reached the socially acceptable height.

\section{EPO-175 Growth \\ Heterozygous GHR gene mutation in a child with idiopathic short stature}

Beatrice Messini'; Sara Pagani'; Cristina Meazza²; Kamilia Laarej; Mauro Bozzola²

${ }^{1}$ Ospedale di Foligno, U.O Pediatrica, Foligno, Italy; ${ }^{2}$ University of

Pavia, Internal Medicine and Therapeutics, Pavia, Italy

Background: Heterozygous growth hormone receptor (GHR) mutations affect receptor function resulting in partial insensitivity to GH. However, some mutations show a greater effect on stature than others leading to different conditions of growth failure.

Objective and hypotheses: We hypothesized that an African girl, presenting with an inexplicable short stature, no dismorphic features or chronic diseases, may have a defect in GHR gene.

Methods: We studied a 18 month old girl with a length of $71.6 \mathrm{~cm}(-2.5$ SDS), a weight of $7200 \mathrm{~g}$, showing a basal serum GH concentration of 4.61 $\mathrm{ng} / \mathrm{ml}$ and IGF-I value of $51.4 \mathrm{ng} / \mathrm{ml}$. Genomic DNA of the patient was extracted, GHR gene was amplified and sequenced on ABI Prism 310 genetic analyzer. Finally we measured quantitatively, by Real-Time PCR, GHR gene expression before and after IGF-I generation test. Variations in input cDNA mass were corrected by normalizing all data with the corresponding GAPDH levels.

Results: No increase in serum IGF-I concentration from basal value (51.4 $\mathrm{ng} / \mathrm{ml})$ to peak level $(45.6 \mathrm{ng} / \mathrm{ml})$ after generation test $(0.025 \mathrm{mg} / \mathrm{Kg} /$ day of $\mathrm{GH}$ for 4 days) was observed A heterozygous mutation resulting in a valine to isoleucine change (V144I) in exon 6 in the extracellular domain of GHR was found in our patient. The quantity of GHR mRNA decreased after IGF-I generation test compared to the basal value.

Conclusions: Our result confirmed previous data showing that GHR gene mutations may cause a short stature. So, these mutations should be considered when other causes of growth failure have been excluded. Further molecular investigations to assess functional activity of this mutation will confirm the involvement of this genomic variation in short stature.

\section{EPO-176 Growth}

\section{Magnetic resonance imaging: the value in diagnosis in children with growth hormone deficiency}

Georgios Kafalidis; Paraskevi Zosi; Georgios Patsouras; Dionisios Karakaidos; Thomais Vlachou; Likourgos Kanaris; Christos Karis

General Hospital Nikaia- Piraeus “Agios Panteleimon”, Department of Pediatrics, Nikaia- Piraeus, Greece

Background: A correct diagnosis of growth hormone deficiency (GHD) is imperative because of the high cost and possible adverse effects of treatment However, the deficiency response criteria and the assay difficulties of the $\mathrm{GH}$ are frequent problems.

Objective and hypotheses: In addition, decreased GH values in obesity and transient GHD in prepubertal children with growth delay can further complicate the diagnosis. Recently there is increasing interest regarding the role of magnetic resonance imaging (MRI) in the diagnosis of the disorder. The purpose of this study was designed to evaluate the diagnostic data provided by MRI in patients with idiopathic growth hormone deficiency (IGHD).

Methods: We study 44 patients, 26 boys and 18 girls aged 5-13 years old with (IGHD) growth hormone peak level $\mathrm{GH}<8 \mathrm{ng} / \mathrm{ml}$ in at least two provocative tests (Clonidine, Insulin or Glucagon test).All the patients was euthyroid and none had diabetic insipidus

Results: The height of the anterior pituitary was $<2$ S.D. for their age in six children ( 5 boys and 1 girl) hypoplasia 13,8\%. In 1 boy there was defected an empty Sella Syndrome $(2,3 \%)$ and in another girl a microadenoma of the pituitary gland $(2,3 \%)$

Conclusions: Accurate evaluation of the height of the anterior pituitary by MRI constitute an additional tool for the diagnosis of IGHD. MRI of pituitary gland can be helpful market during the diagnosis, (cause) and not as routine in all patients with GHD.

\section{EP0-177 Growth \\ Severe growth hormone (GH) deficiency in an Indian child born small for gestational age (SGA)}

Giuseppe Cannalire; Chiara Maria Damia; Maria Piera Ferrarello;

Alessandra Musio; Roberta Pajno; Gisella Garbetta;

Gabriella Pozzobon; Berardo Di Natale; Giuseppe Chiumello

University Vita-Salute S. Raffaele, Department of Pediatrics, Endocrine

Unit, Milan, Italy

Background: In Italy GH treatment is allowed for children born SGA without catch-up growth after the first 4 years of life. In these children a specific hormonal evaluation is not required.

Objective and hypotheses: To show the importance of GH evaluation in a child born SGA

Methods: Adopted SGA and IUGR Indian child (F, 2 years 7 months), the pregnancy was 39 weeks, birth weight $2120 \mathrm{gr}$ (SGA according to Gagliardi et al). At our evaluation pathological weight and lenght for Indian Growth Charts were found (for Italian Growth Charts Cacciari et al.: L -5.3 SDS, BMI -3 SDS). Ketotic hypoglycemias were reported. Endocrine dysfunction was hypothesized. General examination was normal. Clinical features: frontal bossing, triangular face, mild dysmetria of lower limbs and pitched voice. Bone age slightly delayed. General blood tests performed for short stature and infectious diseases evaluation were negative. Karyotype was normal (46 XX). MRI brain: slight disproportion between skull base and cranial vault, Chiari I anomaly, hypoplastic adenohypophysis. Genetic analysis for SRS were not possible (adopted child).

Results: Although GH treatment might have been started after the age of 4 years old, given the clinical features and the anamnestic data, hormonal evaluation was performed: severe isolated GH deficiency was diagnosed (GHRH + ARG peak $0.7 \mathrm{ng} / \mathrm{ml}, \mathrm{IGF}-1<25 \mathrm{ng} / \mathrm{ml}$ ). GH therapy was immediately started (3 years and 10 months) at the dose of $30 \mathrm{mcg} / \mathrm{kg} /$ day. After 6 months catch-up growth $+1,3 \mathrm{SDS}, \Delta \mathrm{GV}+4,9 \mathrm{SDS}$. No more hypoglycaemia were reported.

Conclusions: Current use of National Growth charts is necessary to perform a correct auxological evaluation. When clinical and anamnestic data are suggestive of hormone deficiency, proper exams and specific evaluations must be carried out before starting GH treatment, even in subjects born SGA, in order to make a precise diagnosis and start the required treatment as soon as possible.

\section{EPO-178 Growth \\ The physical, mental and social development and its dependence on the growth of children and the youth \\ Agnieszka Kempa'; Jerzy Szymocha'; Magdalena Jarzynska'; Justyna Kupczynska'; Ewa Barg² \\ ${ }^{1}$ Wroclaw Medical University, Students Science Association of Endocrinology and Diabetology of Adolescence, Wroclaw, Poland; ${ }^{2}$ Wroclaw Medical University, Endocrinology and Diabetology for children and adolescents, Wroclaw, Poland}

Background: Shortness can be defined as the height below 3 percentile according to the current centile charts of the population. The main causes of endocrine short stature are growth hormone deficiency, hypothyroidism and excess of steroid hormones.

Objective and hypotheses: The aim of study was to assess the impact of stature which is lower than the average on aspects of human life such as mood, the way of functioning in society and attitudes to the future.

Methods: The analysis was based on anonymous surveys conducted among children aged 9-18 years as well as their parents. The study group comprised 88 children treated because of the GH deficiency. Parents of patients were also 
surveyed. The comparative group consisted of 130 children.

Results: The average age of the study group was 13.75 .09 years and for the comparative one it was 142.6 years. The mean SDS for growth in the study group is -1.67 and 0.11 in the comparison group. 1/5 study group feel worse because of their height, in comparative group $1 / 10$ children. $71 \%$ of the study group are assessed by the environment as a younger while in the control one such a group includes $21 \%$ children. Patients do not perceive their low growth as a disease. $87 \%$ of their parents recognize the possible impact of the short stature on their children well-being. An average grade achieved at the school for the two groups differ slightly. Nearly $1 / 4$ of the respondents admitted that they are less efficient than they could be when being higher than now. In both groups, over $80 \%$ of respondents believe that they would be able to be successful in the future. The study shows a correlation between a sense of acceptance from peers and the height of patients in the study group.

Conclusions: Short stature does not affect significantly academic performance, physical fitness, contacts with peers, happiness and the other examined aspects of psychomotor development. However, parents note that there could be some possible implications of this health problem to their children life.

\section{EPO-179 Growth \\ A strange picture of karyotype and good response to higher dosage of $\mathrm{GH}$}

Giulia Rodella: Elena Monti; Grazia Morandi; Claudia Piona;

Rossella Gaudino; Franco Antoniazzi

University of Verona, Department of Life and Reproduction, Verona, Italy

Introduction: Genes on Xp chromosome are central for stature. In Turner Syndrome and SHOX mutation GH therapy should generally begin as soon as growth failure occurs and for a good reaction higher doses are required. Monosomy $\mathrm{X}$ is found in about $45 \%$ of patient with Turner syndrome; in the remaining patients is found structural chromosome abnormality or mosaicism. The phenotype of TS is extraordinarily broad. Individuals with a $45, \mathrm{X}$ tend to have a more severe phenotype than those who are mosaic with a normal cell line $(45, \mathrm{X} / 46, \mathrm{XX}$ or $45, \mathrm{X} / 46, \mathrm{XY})$. However, there is no predictable phenotype-genotype correlation.

Case report: We report a 4 years old girl with growth failure: she was small for gestational age (SGA) : BW -2SDS, height -2/-3SDS with a Mid Parent Height $+1 /+2$ SDS; she has been treated with GH therapy $(0.2 \mathrm{mg} / \mathrm{kg} /$ week $)$. As we noted no growth improvement, we performed a chromosome analysis, discovering a picture of Turner-like Syndrome:46 X,Xqter ->Xp22.3->Xq13$>$ Xqter karyotype. So we increased GH therapy $(0.3 \mathrm{mg} / \mathrm{kg} /$ week $)$ and statural growth passed from $-2,5$ to M SDS (+ 2,5 Delta SDS). To explain the surprising growth enhancement we performed FISH analysis with specific probe for centromere and subtelomeric regions of X chromosome long arm (Xq) and short arm (Xp) and MLPA analysis for subtelomeric region in particular SHOX gene. We found two centromeres and a partial duplication of Xq and there was no SHOX gene deficit.

Conclusion: We are going to perform other genetic analyses (such as array$\mathrm{CGH}$ ) for the understanding of the low stature and of the very good answer to GH therapy and besides we must remember that sometimes phenotype of duplication syndromes is similar to the deletion ones.

\section{EPO-180 Growth}

Is it a case of idiopathic short stature?

Francesca Simi'; Maria Francesca Tutera'; Giuseppe Saggese 2

${ }^{1}$ University of Pisa, Pediatrics, Monsagrati Lucca, Italy; ${ }^{2}$ University of

Pisa, Pediatrics, Pisa, Italy

Background: Jade is a 9 years old girl who comes to our attention for short stature and for a progressive slowing of growth rate since preschool age. Objective and hypotheses: On examination, Jade shows an height of 120.5 $\mathrm{cm}(-1.6 \mathrm{SDS})$, a growth rate of $2 \mathrm{~cm} /$ year in the last year and a genetic target of $160.3 \mathrm{~cm}(-0.3 \mathrm{SDS})$. We find a slight varus deformity of the lower limbs. The child has a BMI above 50 percentile.Blood examination is performed: complete blood count, electrolytes, ferritin, screening for celiac disease, evaluation of thyroid function, IGF-1, urinalysis, stimulation tests for growth hormone and karyotype. The results of the blood tests are normal. The radiograph of the wrist and hand shows bone age corresponding to chronological age. Methods: Before labeling Jade as a case of idiopathic short stature, we reex- amine the patient in order to make a more detailed anthropometric evaluation. Arm span $(118.5 \mathrm{~cm}) /$ stature $(120.5 \mathrm{~cm})$ is in a normal ratio $(0.98)$. Sitting height/stature ratio $(0.58)$ is altered as well as the ratio between upper and lower segment of the body. There is also a mild muscle hypertrophy.

Results: In the suspicion of a SHOX gene haploinsufficiency short stature associated with a slight disproportion between upper and lower body segment we suggest to performe the molecular analysis of SHOX gene. MPLA analysis shows the deletion of part of the region PAR (Xp22.32). including some of the regulatory regions of the gene but not the gene itself. The microsatellite analysis reveals the lack of paternal contribution.

Conclusions: The deletion found in Jade is similar to the deletion present in another patient Arianna, we previously have seen. Arianna, at the chronological age of 14 years has a stature of $141 \mathrm{~cm}$, equal to -2.8 SDS, with an obvious alteration of body proportions, mesomelia and Madelung deformity, typical of the Leri-Weill syndrome. Jade and Arianna are currently treated with growth hormone and will be regularly monitored to assess growth and evolution of anthropometric abnormalities.

\section{EPO-181 Growth \\ Evaluation of short children referred by school screening: an analysis of 2589 children according to the WHO norms of 2007}

Feriel Limam ${ }^{1}$; Zinnet Turki'; Sihem Saad Allah²; Ines Kamoun²;

Olfa Larbech'; Chiheb Ben Rayana ${ }^{3}$; Chiraz Bouzid;

Claude Ben Slama²; Ikram Louati ${ }^{4}$

'Grombalia Hopital, Pediatric, Nabeul, Tunisia; ${ }^{2}$ National Nutrition

Institut, Endocrinology and Metabolic desease, Tunis, Tunisia; ${ }^{3} \mathrm{National}$ Nutrition Institut, Medical Laboratory, Tunis, Tunisia; ${ }^{4} \mathrm{Grombalia}$

Hopital, Pediatrics, Nabeul, Tunisia

Background: An isolated delay in growth can reveal a treatable pathology, a school inquiry is launched.

Objective and hypotheses: The objective is to identify children with treatable deseases.

Methods: Evaluation included: physical examination, weight and height. For the short children: heights were below -2DS ,we invite the parents for a detailed medical history, nutritional inquiry and laboratory tests successively according to primary results: blood count, bone age, thyroid function, celiac serology, growth hormone screening, and chromosome tests. We use the new tables of the WHO published in 2007 (height/age/sex).

Results: The results were: $35(1.3 \%)$ have short height:Constitutional delay (17children), intra-womb short stature (9children), growth hormone deficiency (4children), thyroid dysfunction ( 2 children), Bardet Biedel syndrome (a child), bone constitutional disease (a child), congenital hepatic disease (a child).

Conclusions: We conclude the majority of short stature in our region is due to normal variation although potentially treatables causes account for at least $2.3 \%$ of the children and merit a systemic screening to identify infants with short stature of potentially treatable etiology.

\section{EP0-182 Growth \\ Atypical presentation of hypercortisolism in two adolescents with anorexia nervosa leading to delayed diagnoses \\ Marie Devernay; Sébastien Rouget; Patrick Alvin \\ Hôpital Kremlin-Bicêtre, Service de médecine pour adolescents, Le Kremlin-Bicetre, France}

Background: Anorexia nervosa, because of its repercussions on organism in growth, can hide or attenuate signs of other diseases interacting with growth and puberty.

Cases report: We reported two cases of adolescents (Nathalie and Julie) with anorexia nervosa who had primary amenorrhea and stopped growing (first consultation at 16 and 12 years respectively). But, whereas Body Mass Index were progressively normal, growth and puberty still stopped during almost 2 years, hidden anorexic hyperactivity were then suspected.

Methods: But, finally, because of a buffalo hump, swelling of the neck and the face, supraclavicular fat pads and hypertension for Nathalie and apparition of a moon facies for Julie, the diagnostic of hypercortisolism was suspected and confirmed by biology. 
Results: Nathalie had an ACTH-dependant Cushing's syndrome due to pituitary corticotroph microadenoma. Hypercorticism completely disappeared after surgery and menstres appeared. Julie had an ACTH-independent Cushing's syndrome. Dexamethasone tests showed a paradoxical answer with cortisol increase. These results were very evocative of a rare illness, the PPNAD (Primary Pigmented Nodular Adrenocortical Disease).

Ultrasonography, scanner and MRI showed normal adrenals but iodocholesterol scintigraphy showed an isolated bilateral adrenal uptake. Mutation of the gene of the protein PRKAR1A (in 17q22-24), regulating sub-unit (R1A) of the Protein Kinase A, was present.

Moreover Julie had some lentigines, so the diagnosis of "Carney complex" was made. A bilateral surrenalectomy was then performed, confirming in histology the diagnosis. Treatment with hydrocortisone and fludrocortisone was necessary for all life.

Conclusions: In anorexia nervosa, patients who took back a normal BMI, a complete endocrinal balance sheet have to be performed, if the growth stopped and/or puberty stopped beyond habitual delays ( 2 years maximum).

\section{EPO-183 Growth}

\section{Noonan syndrome in the father and son}

Laura Gutiérrez Pascual'; Luis Salamanca Fresno';

Fernando Santos 2 ; Sixto García Miñaur²; Ricardo Gracia Bouthelier'; Isabel González Casado ${ }^{1}$

'La Paz Children's Hospital, Endocrinology Unit, Madrid, Spain; ' $2 a$

Paz Children's Hospital, Genetic Unit, Madrid, Spain

Background: Noonan syndrome (NS) is a quite prevalent syndrome characterized by short stature, facial, skeletal and cardiovascular abnormalities and cryptorchidism. PTPN11 mutations account for $50 \%$ of cases and promote non-receptor protein tyrosine phosphatase (SHP-2) gain of function through the RAS/MAPK pathway, which has been implicated in a wide variety of cancers.

Objective and hypotheses: We submit two NS cases

Methods: We report on a 7 months old boy who has clinical features consistent with NS (short stature, typical facial features and cryptorchidism).

His father had been diagnosed in infancy with Dubowitz syndrome and had received treatment with growth hormone $(\mathrm{GH})$ during eight years.

On examination, he has typical facial features of NS too, and has recently developed a lymphoma. Besides this, he had suffered a scrotal lymphangioma several years ago, which is a characteristic feature of NS.

Results: In the genetic study, both are heterozygotes for the E139D (c. $417 \mathrm{C}>\mathrm{G}$ ) mutation in exon 4 of the PTPN11 gene, thus confirming the NS. Currently, at 2 years old, our patient's height is much lower of percentile three, in $-5.3 \mathrm{SD}$, and he has a normal psychomotor development.

Conclusions: GH treatment has been proved to improve growth in some of these patients. The E139D mutation is not known to increase cancer risk in NS patients, but has been previously reported in two patients with NS who developed a cerebral glioma. It is difficult to value if exist any relationship between this mutation and the GH treatment with the development of the lymphoma in our patient's father. At this point we wonder, is the GH treatment safe in our patient?

\section{EPO-184 Growth}

\section{Noonan syndrome; clinical, biological and genetic aspects}

Meriem Bensalah ${ }^{1}$; Helene Cave ${ }^{2}$; Mehdi Chamouni'; Zahra Kemali'

${ }^{1}$ Central Hospital of Army, Endocrinology Department, Algiers, Algeria;

${ }^{2}$ Robert Debre Hospital, Genetic Department, Paris, France

Background: Noonan syndrome(NS) is one of the most common syndromes transmitted by a mendlian mode. 'The incidence of affected individuals is estimated to be between $1 / 1000$ and $1 / 2500$. NS is characterized by dysmorphic facial features, congenital heart disease(pulmonary valve stenosis, hypertrophic cardiomyopathy and atrial septal defects), post natal short stature, neck and chest abnormalities. Mental retardation, bleeding abnormalities, hearing difficulty and cryptorchidism are also occasionally observed in affected individuals. The last few years germeline mutations in several genes have been found in people with clinical signs of NS, accounting for approximately $70 \%$ of cases. Heterozygous mutations in nine genes have been documented: PTPN11,KRAS, NRAS,SOS1,RAF1,BRAF,SHOC2,MEK1. CBL gene germeline mutations have been recently associated with NS phenotype.
Objective and hypotheses: We report six cases of Noonan syndrome and describe clinical, biological and genetic characteristics.

Methods: Clinical diagnostic for Noonan syndrome was established according to Van Der Burgt Criteria. All patients have blood numeration, ECG, cardiac ultrasound, Hearing evaluation, intellectual quotient evaluation, Thyroid function (FT4, TSHus) screening and IGF1 measurement.

Results:

\begin{tabular}{|c|c|c|c|c|c|c|}
\hline & Case 1 & Case 2 & Case 3 & Case 4 & Case 5 & Case 6 \\
\hline $\begin{array}{l}\text { Age at diagnosis } \\
\text { (years) }\end{array}$ & 11 & 5 & 12 & 3 & 1,5 & 22 \\
\hline Sex & M & M & $\mathrm{F}$ & M & M & $\mathrm{F}$ \\
\hline Birth weight (g) & 2200 & 2900 & 3100 & 2700 & 3200 & 3500 \\
\hline consanguinity & - & $t$ & - & - & - & - \\
\hline Dysmorphic face & t & $t$ & $\dagger$ & $\dagger$ & $\dagger$ & $\dagger$ \\
\hline Chest deformities & $\begin{array}{l}\text { Pectus carinatus, } \\
\text { pectus excavatum. }\end{array}$ & $\begin{array}{l}\text { Pectus carinatus, } \\
\text { pectus excavatum. }\end{array}$ & - & - & - & $\leq$ \\
\hline Height & $-\mathrm{P} 3$ & $-\mathrm{P} 3$ & $-\mathrm{P} 3$ & $-\mathrm{P} 3$ & $-\mathrm{P} 3$ & $-\mathrm{P} 3$ \\
\hline Mental retardation & $t$ & $\dagger$ & $\dagger$ & $\dagger$ & $t$ & $\dagger$ \\
\hline Heart disease & $\begin{array}{l}\text { Percistent arteriel } \\
\text { canal }\end{array}$ & pulmonic stenosis & $\begin{array}{l}\text { mitral } \\
\text { prolapse }\end{array}$ & $\begin{array}{l}\text { aortic } \\
\text { stenosis }\end{array}$ & - & $\begin{array}{l}\text { mitral } \\
\text { stenosis }\end{array}$ \\
\hline Cryptorchidism & $\dagger$ & $\dagger$ & & $t$ & $\dagger$ & \\
\hline Hearing defects & - & - & $\dagger$ & - & - & $\dagger$ \\
\hline $\begin{array}{l}\text { Hematological } \\
\text { abnormalities }\end{array}$ & - & - & - & $\begin{array}{l}\text { monocy- } \\
\text { tosis }\end{array}$ & JMML & - \\
\hline Genetic mutations & - & - & $\begin{array}{c}\text { CBL } \\
\text { germeline } \\
\text { mutation }\end{array}$ & - & $\begin{array}{l}\text { PTPN11 } \\
\text { germeline } \\
\text { mutation }\end{array}$ & $\begin{array}{c}\text { not done } \\
\text { yet }\end{array}$ \\
\hline GH treatment & $\dagger$ & $\dagger$ & - & - & - & - \\
\hline
\end{tabular}

Conclusions: Clinical diagnosis of NS was easy in our patients, most of them presented major criteria (short stature, congenital cardiopathy, mental retardation, cryptorchidism). Genetic investigation show CBL mutation in patient 3 which may induce JMML. The presence of monocytosis may induce JMML in patient 4 . GH treatment improve stature in the children treated, unfortunately it was not indicated in those with CBL mutation, monocytosis and JMML.

\section{EPO-185 Hypoglycaemia}

\section{Long-term outcome of a child with hyperinsulinism-hyperammonaemia syndrome} Ho-chung Yau; Gary Wing-kin Wong

Department of Paediatrics, Prince of Wales Hospital, The Chinese

University of Hong Kong, Shatin, New Territories, Hong Kong

Background: Clinical features of hyperinsulinism-hyperammonaemia syndrome are variable. A potential genotype-phenotype correlation of GLUD1 mutation and clinical features has been suggested.

Objectives and hypotheses: We report the long-term outcome of a child with hyperinsulinism-hyperammonaemia syndrome.

Methods: A male infant was born at 38 gestational weeks with birth weight of $3.605 \mathrm{~kg}$. He presented with convulsion at 28 days of life. Physical examination was unremarkable. He was initially treated as meningoencephalitis. CT brain was unremarkable. Bacterial and viral cultures were negative. Plasma glucose upon admission was $1.2 \mathrm{mmol} / 1$ with negative urine ketone. Glucose infusion was high up to $12.1 \mathrm{mg} / \mathrm{kg} / \mathrm{min}$. Two sets of critical samples were obtained during hypoglycaemia.

\begin{tabular}{lcc|} 
Date & $\mathbf{2 8}$ days & $\mathbf{3 4}$ days \\
\hline Plasma glucose $(\mathrm{mmol} / \mathrm{l})$ & 1.9 & 2.0 \\
\hline Insulin $(\mathrm{mlU} / \mathrm{l})$ & 23.9 & 10.5 \\
\hline Growth hormone $(\mathrm{IU} / \mathrm{l})$ & 54 & \\
Cortisol $(\mathrm{mmol} / \mathrm{l})$ & 311 &
\end{tabular}

Workup for metabolic diseases was unremarkable. But ammonia levels were persistently high up to $252-353 \mathrm{umol} / \mathrm{l}$. Octreotide injection was started to achieve euglycaemia. Protein free diet was tried but without success in lowering ammonia levels. Octreotide was later switched to oral diazoxide and hydrochlorothiazide. Full enteral feeding was established with stable glucose levels.

Results: Genetic analysis showed a mutation in axon 10; Asn410I1e (N410I), a mutation of AAC to ATC at codon 410. The diagnosis of hyperinsulinism- 
hyperammonaemia syndrome was confirmed. We follow the child until he is 6 years of age. Home blood glucose monitoring prevented him from hypoglycaemia. Fasting test was performed every year to fine tune his diazoxide dosage. He remained seizure free while ammonia levels remained high up to 123-218 umol/1. Formal developmental assessment performed at 5 years of age showed delay in cognitive and speech development for 1-1.5 years, and attention-deficit hyperactivity syndrome.

Conclusions: Clinical features of hyperinsulinism-hyperammonaemia syndrome are variable. A genotype-phenotype correlation requires confirmation in larger series of patients.

\section{EPO-186 Hypoglycaemia \\ Hyperinsulinaemic hypoglycaemia associated with early onset brain injury and cerebral visual impairment \\ Anitha Kumaran ${ }^{1}$; Clare Gilbert'; Kate Morgan ${ }^{1}$; Louise Hinchey'; Khalid Hussain' \\ ${ }^{1}$ Great Ormond Street Hospital NHS trust, Paediatric Endocrinology, \\ London, United Kingdom; ${ }^{2}$ Institute of Child Health, Clinical and \\ Molecular Genetics Unit, Iondon, United Kingdom}

Background: Hyperinsulinaemic hypoglycaemia $(\mathrm{HH})$ is a major cause of brain injury due to the lack of alternate cerebral fuels in the form of ketone bodies, as insulin suppress lipolysis and ketogenesis.

Objective and hypotheses: To describe clinical and neuroimaging features of 4 patients with $\mathrm{HH}$ and early onset brain injury.

Methods: All 4 patients ( 2 male, 2 female) were born at term. Mean birth weight was 3207 grams (range 2730 - 3500 grams). All 4 were discharged home between days 1-2 of life and presented to local hospital between day 3-5 of life with poor feeding and seizures.

Results: Biochemical investigations confirmed HH. All had diazoxide responsive $\mathrm{HH}$, although $\mathrm{HH}$ was transient in 2 patients and persistent in 2 patients. All 4 patients had neonatal seizures requiring antiepileptic treatment. 2 patients have ongoing seizures requiring antiepileptic medication. Magnetic resonance imaging (MRI) was performed at a mean age of 9.25 days (Day 6-14).Patient 1 and 2 have bilateral involvement of parieto-occipital cortical and subcortical regions. Of these neurodevelopmental data is not available in 1 patient (acute management ongoing) and in 1 patient (transient $\mathrm{HH}$ ), there are no neurodevelopmental concerns. Patient 3 (transient HH) had left parieto-occipital abnormality initially and a subsequent MRI scan at 6 months showed extensive volume loss in left cerebral hemisphere and small left thalamus. Patient 4 has involvement of the thalamus in addition to bilateral parietooccipital cortical and subcortical abnormality. These 2 patients have global developmental delay, abnormal visual evoked potentials and visual impairment and microcephaly (head circumference $<10$ th centile).

Conclusions: This is the first study showing that profound hypoglycaemia secondary to $\mathrm{HH}$, even of short duration (48 hours) can be associated with early MRI evidence of parieto-occipital, cortical, subcortical as well as thalamic brain injury. These observations have important implications for detecting and managing patients with $\mathrm{HH}$

\section{EPO-187 Hypoglycaemia \\ $45, X / 46, X, r(X)$ mosaicism causing Kabuki-like syndrome with short stature and congenital hyperinsulinism missing further signs of Turner syndrome}

Isabel Wagner'; Johannes Weigel'; Skadi Beblo'; Dorothea Wand; Antje Körner'; Wieland Kiess'; Roland Pfaeffle

'University of Leipzig, Dept. of Women's \& Child Health, Leipzig,

Germany; ${ }^{2}$ University of Leipzig, Institute for Human Genetics,

Germany, Germany

Background: About $6 \%$ of patients with Turner syndrome (TS) have ring chromosomes, often as $45, \mathrm{X} / 46 \mathrm{X}, \mathrm{r}(\mathrm{X})$ mosaicism. In rare instances, these patients have been reported to resemble patients with Kabuki syndrome in the absence of further typical TS features. This can be explained by a loss of the $\mathrm{X}$ inactivation center (XIST) on the ring chromosome. Here, we present a patient with $45, \mathrm{X} / 46, \mathrm{X}, \mathrm{r}(\mathrm{X})$ mosaicism and Kabuki-like syndrome showing significant postnatal hyperinsulinism and growth retardation.

Patient information: The Patient was born preterm (32 weeks of gestation) with intrauterine growth retardation (1376 g/-3.10 SDS, $40 \mathrm{~cm} /-4.92$ SDS). First episodes of hypoglycaemia were noticed in the hospital due to bronchiolitis during infancy. Developmental milestones were slightly retarded and she developed seizures starting at two years of age. Several nocturnal episodes of hypoglycaemia were observed in the presence of elevated fasting insulin concentrations. The patient responded extremely well to diazoxide treatment. Results: Genetic analysis revealed a chromosomal mosaic (45,X $(60 \%) / 46, X, r(X))$. Both parents showed a normal karyotype. In our patient, Kabuki-like syndrome is caused by the loss of XIST. She is growing constantly below the 3rd percentile (-2.41 SDS/P0.9) and below her parental target height. As the SHOX gene escapes X-inactivation it remains questionable whether her growth will resemble that of patients with typical TS and whether she may require future hGH (growth hormone) treatment.

Conclusion: Interestingly our patient did have an associated congenital hyperinsulinism. Treatment with diazoxide has led to an extreme response with a strong increase of blood sugar levels. Already under a really small dose she has stable glucose and insulin levels and she did not have any further hypoglycaemias or seizures. Kabuki-like syndrome is a very rare condition and hence endocrine guidelines do not exist. We speculate that patients, with a Kabuki-like syndrome, could also benefit from hGH therapy even if there is no hGH deficiency.

\section{EP0-188 Perinatal and Neonatal Endocrinology \\ Relations of estimated birth brain weight (BRW) to circulating Insulin-like Growth Factor-I in not-life-threatened newborns: an explanatory role for the part of birth body weight not attributable to BRW is recognizable independently of the presence of Caesarean Section \\ Cesare Terzi'; Raffaele Virdis'; Werner F. Blum²; Sergio Zani3; Marco Riani3; Gabriele Tridenti4; Andrea Cerioli3; Elena Chesi ${ }^{5}$; Sergio Bernasconi'; Giacomo Banchinis \\ ${ }^{1}$ University of Parma, Department of Pediatrics, Parma, Italy; 'University of Giessen, Department of Pediatrics, Giessen, Germany; ${ }^{3}$ University of Parma, Department of Economics, Parma, Italy; ${ }^{4} \mathrm{~S}$. Maria Nuova Hospital, Department of Obstetrics, Reggio Emilia, Italy; ${ }^{5}$ S. Maria Nuova Hospital, Department of Pediatrics, Reggio Emilia, Italy}

Background: The involvement of fetal head in delivery mechanics is profoundly altered by Caesarean Section (CS).

Objective and hypotheses: We evaluated the explanatory role of the part of birth body weight (BW) not attributable to brain (NBBW) in relations of estimated birth brain weight (BRW) to human newborn (NWB) blood serum Insulin-like Growth Factor-I (IG1) in presence of controls including CS.

Methods: IG1 was measured by radioimmunoasay in $\mu \mathrm{M} / \mathrm{dL}$ in each NWB at one of the first 5 days of postnatal life (x), 5 days after $\mathrm{x}(\mathrm{y})$ and 10 days after $x(z)$. Gender (SEX), gestational age at birth (GA), CS, BW, head circumference $(\mathrm{HC})$, postnatal age at $\mathrm{x}$ (PNA), as well as the presence of a birth body weight (BW) <10.th centile for GA (SGA) and of preterm birth, defined as $\mathrm{GA} \leq 36$ complete weeks (PTB), were recorded. 78 NWBs with complete data (females, $\mathrm{n}=35$; males, $\mathrm{n}=43$; GA range: $28-42$ weeks; $\mathrm{BW}$ range $1200-4150$ gr; SGA, n=20; PTB, n=46; CS, n=52)(computations; SEX, male:1, female:0; PTB, CS and SGA, condition present:1, condition absent:0) and without any among life-threatening disease, diabetes mellitus (DM) or mother with DM were included in the study. BRW was calculated, according to McLennan and Lindley, through the following formula: $\mathrm{BRW}=0.037 \mathrm{x} \mathrm{HC}^{2.57}$, while NBBW was calculated as BW minus BRW (units; BW, BRW and NBBW: gr; HC: $\mathrm{cm})$. Natural log-transformed IG1 (IG1-NL) resulted near-normally distributed. Multiple linear regression (MLR) was used.

Results: Table 1 shows MLR partial correlation coefficient $(r)$ and $t$ value of the BRW partial correlation with IG1-NL, F and R2 of corresponding MLR model and respective significances regarding MLR models bearing IG1-NLX, IG1-NLy or IG1-NLz as outcome and as predictors either 1) BRW, SEX, SGA, PTB, CS and PNA (Table 1A), or 2) BRW, SEX, NBBW, PTB, CS and PNA (Table 1B). 


\begin{tabular}{|c|c|c|c|c|c|c|c|c|c|c|}
\hline A) & vs. & IG1-LNx & IG1-LNy & IG1-LNz & II & B) & vs. & IG1-LNx & IG1-LNy & IG1-LNz \\
\hline \multirow[t]{3}{*}{ BRW } & $r / t$ & $\begin{array}{c}.282 / \\
2.475 a\end{array}$ & $\begin{array}{c}.314 / \\
2.783 b\end{array}$ & $\begin{array}{c}.251 / \\
2.181 a\end{array}$ & II & BRW & $r / t$ & $\begin{array}{c}-.015 / \\
-0.125 n s\end{array}$ & $\begin{array}{c}.089 / \\
0.753 \mathrm{~ns}\end{array}$ & $\begin{array}{c}.029 / \\
0.248 \mathrm{~ns}\end{array}$ \\
\hline & $\mathrm{F}$ & $4.943 \mathrm{c}$ & $10.098 \mathrm{c}$ & $5.258 \mathrm{c}$ & II & & $\mathrm{F}$ & $7.487 \mathrm{c}$ & $11.302 \mathrm{c}$ & $6.507 \mathrm{c}$ \\
\hline & $\mathrm{R} 2$ & .295 & .460 & .308 & /I & & $\mathrm{R} 2$ & .388 & .489 & .355 \\
\hline Table & & $\begin{array}{l}\text { Signi- } \\
\text { ficances; }\end{array}$ & $\begin{array}{c}a, \\
p<.050 ;\end{array}$ & $\begin{array}{c}b \\
p<.010\end{array}$ & $\begin{array}{c}c, \\
p<.001 ;\end{array}$ & $\begin{array}{l}\text { ns, not } \\
\text { significant. }\end{array}$ & & & & \\
\hline
\end{tabular}

Conclusions: BRW relations to IG1-LN not fully explained 1) by CS, SEX, PTB and SGA, possibly accounting for pathologies related to birth size/birth mechanics, or 2) by PNA could have been sustained by NBBW in studied not-life threatened NWBs.

\section{EPO-189 Perinatal and Neonatal Endocrinology \\ Relations of gestational age at birth to the ratio between serum levels of Insulin-like Growth Factor (IGF)-II and IGF Binding Protein-3 in the not-life-threatened newborn: relevance of birth body size beyond the presence of a small birth body weight for gestational age \\ Cesare Terzi'; Werner F. Blum²; Sergio Zani3; Marco Riani;; \\ Gabriele Tridenti'; Andrea Ceriolï; Lidia Garavelli5; Sergio Bernasconi'; Raffaele Virdis'; ${ }^{1}$ Giacomo Banchini ${ }^{5}$ \\ 'University of Parma, Department of Pediatrics, Parma, Italy; 'University of Giessen, Department of Pediatrics, Giessen, Germany; ${ }^{3}$ University of Parma, Department of Economics, Parma, Italy; ${ }^{4} \mathrm{~S}$. Maria Nuova Hospital, Department of Obstetrics, Reggio Emilia, Italy; ${ }^{5}$ S. Maria Nuova Hospital, Department of Pediatrics, Reggio Emilia, Italy}

Background: Birth body weight (BW) has been related negatively to the ratio between Insulin-like Growth Factor (IGF)-II and IGF Binding Protein-3 (IGFBP-3) blood serum levels (IG2/IB3) in men.

Objective and hypotheses: We evaluated the role of BW in explaining relations of gestational age at birth (GA; unit:complete week) to human newborn (NWB) IG2/IB3 besides possible influences of gender (SEX) and of the presence of a $\mathrm{BW}<10$.th centile for GA (SGA).

Methods: Blood serum IGF-II (IG2) and IGFBP-3 (IB3) were measured by radioimmunoasay in $\mu \mathrm{M} / \mathrm{dL}$ in each NWB at one of the first 5 days of postnatal life (x), 5 days after $\mathrm{x}(\mathrm{y})$ and 10 days after $\mathrm{x}(\mathrm{z})$. SEX, GA, BW, postnatal age at $x$ (PNA), and the presence of preterm birth (PTB; definition: $\mathrm{GA} \leq 36$ complete weeks) and of SGA were recorded. $78 \mathrm{NWBs}$ with complete data (females, $\mathrm{n}=35$; males, $\mathrm{n}=43$; GA range: $28-42$; $\mathrm{BW}$ range $1200-4150 \mathrm{gr}$; $\mathrm{SGA}$, $\mathrm{n}=20 ;$ PTB, $\mathrm{n}=46$ ) and without any among life-threatening disease, diabetes mellitus (DM) or mother with DM were included in the study (computations; male:1; female:0; SGA present:1; SGA absent:0). IG2/IB3, calculated as IG2 through chronologically corresponding IB3, was standardized according to the transformation of Van der Waerden (IG2/IB3-S). IG2/IB3-S resulted nearnormally distributed. Spearman's rank correlation (SC) and multiple linear regression (MLR) were used.

Results: Correlation coefficients regarding SCs of BW with IG2/IB3 at x, $\mathrm{y}$ and $\mathrm{z}$ were resp. 1) -.47, -.46 and -.54 in females (p, .0042-.0008), and -2 ) $-.35,-.46$ and -.40 in males (p, .0234-.0019). Table 1 shows MLR partial correlation coefficient $(r)$ and $t$ value of partial correlations of GA with IG2/IB3$\mathrm{S}, \mathrm{F}$ and R2 of corresponding MLR model and resp. significances regarding MLR models bearing IG2/IB3-Sx, IG2/IB3-Sy or IG2/IB3-Sz as outcome and as predictors either 1) GA, SEX, SGA and PNA (Table 1A), or 2) GA, SEX, BW and PNA (Table 1B)

\begin{tabular}{|c|c|c|c|c|c|c|c|c|c|c|}
\hline A) & vs. & $\begin{array}{c}\text { IG2I } \\
\text { IB3-Sx }\end{array}$ & $\begin{array}{c}\text { IG2/ } \\
\text { IB3-Sy }\end{array}$ & $\begin{array}{c}\text { IG2I } \\
\text { IB3-Sz }\end{array}$ & II & B) & vs. & $\begin{array}{c}\text { IG2I } \\
\text { IB3-Sx }\end{array}$ & $\begin{array}{c}\text { IG2I } \\
\text { IB3-Sy }\end{array}$ & $\begin{array}{c}\text { IG2I } \\
\text { IB3-Sz }\end{array}$ \\
\hline \multirow[t]{3}{*}{ GA } & $\mathrm{r} / \mathrm{t}$ & $\begin{array}{c}-344 / \\
-3.130 \mathrm{a}\end{array}$ & $\begin{array}{r}-347 / \\
-3.163 b\end{array}$ & $\begin{array}{r}-397 / \\
-3.700 c\end{array}$ & /I & GA & $\mathrm{r} / \mathrm{t}$ & $\begin{array}{c}-.020 / \\
-0.173 \mathrm{~ns}\end{array}$ & $\begin{array}{c}.028 / \\
0.238 \mathrm{~ns}\end{array}$ & $\begin{array}{c}-.036 / \\
-0.309 \mathrm{~ns}\end{array}$ \\
\hline & $\mathrm{F}$ & $6.752 \mathrm{c}$ & $4.643 b$ & $4.367 \mathrm{~b}$ & II & & $\mathrm{F}$ & $6.014 c$ & $4.671 b$ & $5.210 \mathrm{~b}$ \\
\hline & R2 & .270 & .203 & .193 & /I & & R2 & .248 & .204 & .222 \\
\hline Table 1. & & $\begin{array}{l}\text { Signi- } \\
\text { ficances; }\end{array}$ & $\begin{array}{c}\mathrm{a}, \\
p<.0050 ;\end{array}$ & $\begin{array}{c}\text { b, } \\
\text { p<.0025; }\end{array}$ & $\begin{array}{c}c, \\
p<.0005 ;\end{array}$ & $\begin{array}{l}\text { ns, not } \\
\text { significant. }\end{array}$ & & & & \\
\hline
\end{tabular}

Conclusions: BW could be involved in inverse GA relations to IG2/IB3-S otherwise not explained by SEX, SGA and PNA as such in NWBs free of life-threatening illness.

\section{EPO-190 Perinatal and Neonatal Endocrinology Pseudohypoaldosteronism: case report}

\section{Liliana Mejia de Beldjenna'; Audrey Mary Matallana²;}

Juan Pablo Llano ; Jaime German Gonzalez"; Consuelo De Roveto

${ }^{1}$ Clinica Valle del LILI,Fundación Infantil Club NoeL,Universidad Libre, Valle, Cali, Colombia; ${ }^{2}$ Universidad del Valle, Valle, Cali, Colombia; ${ }^{3}$ Hôpital Femme Mere Enfant Lyon, Rodano, Lyon, France; ${ }^{4}$ Centro Medico Imbanaco, Valle, Cali, Colombia

Background: Congenital Pseudohypoaldosteronism was first reported by Check and Perry in 1908. This syndrome is caused by alterations of high affinity type I aldosterone receptors of kidney, leucocytes and other tissues. It is characterized by sodium loss, hyponatremia, hypovolemia, hyperkalemia and metabolic acidosis. Plasma aldosterone and renin are increased. There are two types renal and systemic. Treatment is with spironolactone or fluorinated steroids.

Objetive: To describe a patient with pseudohypoaldosteronism including his management.

Methods: To describe un patients with Pseudohypoaldosteronism.

Results: Term Newborn with a serum potassium of greater than $7 \mathrm{mmol} / \mathrm{l}$, sodium $124 \mathrm{mmol} / 1$, plasma aldosteron $4,7 \mathrm{nmol} / 1(\mathrm{vn} 0.14-1,7 \mathrm{nmol} / 1)$, plasma renin $59,5 \mathrm{pg} / \mathrm{ml}$ ( vn2,4-21.9 pg/ml), normal blood pressure, serum cortisol and 17 hydroxyprogesterone and normal renal echography and function. $\mathrm{Pa}-$ tient was treated for pseudohypoaldosteronism with fludrocortisones $(0.3 \mathrm{mg} /$ day). Genetic study is pending.

Conclusion: Type I renal pseudo hypoaldosteronism is inherited as an autosome dominant trait, with renal sodium losses and hyperkalemia. It may be asyntomatic and the renal defect may improve with time with a better response to mineralocorticoids. Genetic studies show loss of function mutation s of chromosome 4q31,1 of distal and collector renal tubules.

\section{EPO-191 Perinatal and Neonatal Endocrinology \\ Adiponectin and leptin levels of large for gestational age (LGA) newborns with different weight gain during the first month of life Angelika Solntsava \\ Belarus Stage Medical University, Pediatrics, Minsk, Belarus}

Background: Leptin and adiponectin are adipocit-secreted hormones that regulate energy homeostasis and metabolism.

Objective and hypotheses: To determine the dynamic of the levels of adiponectin and leptin of LGA newborns during the first month of life and to establish the relationship of these parameters with their physical development.

Methods: 77 term infants LGA (birth weight $4270.0 \pm 0.22 \mathrm{~g}$ ) were divided in to two groups according to the weight gain during the first month of life: more than $1 \mathrm{~kg}(\mathrm{n}=34)-1$ st group, less than $1 \mathrm{~kg}(\mathrm{n}=43)$ 2nd group. Weight, body mass index (BMI), ponderal index (PI) at birth in both groups were similar. Clinical and laboratory examination, adiponectinemia and leptinemia at 1,6 and 30 days of life were perfomed. Adipocytocines were measured by immunosorbent assay (ELISA). Statistical data processing was performed using Statistica 6.0 with elements of SPSS 17

Results: Weight gain during the 1st month of life in group 1 was $1.2 \pm 0.21$, in group $2-0.7 \pm 0.23 \mathrm{~kg}(\mathrm{p}=0.0001)$. Breast feeding in the 1 st group received $70.6 \%$, in the 2 nd $-51.2 \%$. Significant differences in content of adiponectin level in infants we have not found ( $\mathrm{p}=0.404-0.933)$, but correlation between PI and adiponectin level at 1 month $(\mathrm{r}=-0.560, \mathrm{p}=0.024)$ was defined. The concentration of leptin in children with greater weight gain at 1 month of life was significantly higher $(133.4 \pm 42.45 \mathrm{ng} / \mathrm{ml})$ than in group $2(74.04 \pm 35.59 \mathrm{ng} /$ $\mathrm{ml}, \mathrm{p}=0.001$ ).

Conclusions: Hyperleptinemia and negative correlation link between PI and adiponectin levels in LGA newborns with a weight gain more than $1.0 \mathrm{~kg}$ during the first month of life, can be used as a predictor of unfavorable metabolicbeing in the future. 


\section{EPO-192 Perinatal and Neonatal Endocrinology}

Protein changes in the serum of newborns with intrauterine growth restriction

\section{$M^{a}$ Dolores Cañete ${ }^{1}$; $M^{a}$ Dolores Ruiz-González ${ }^{2}$;}

Jose L Gomez-Chaparro ; Juan L Lopez-Barea3; Ramón Cañete ${ }^{2}$

${ }^{1}$ University of Córdoba, Grupo PAIDI CTS-329. IMIBIC, Córdoba,

Spain; ${ }^{2}$ Hospital Universitario Reina Sofía, Pediatric Unit, Cordoba,

Spain; ${ }^{3}$ Universidad de Cordoba, Departament of Biochemistry and

Molecular Biology, Cordoba, Spain

Objectives: To compare the total serum protein content in newborns (NB) with intrauterine growth retardation (IUGR) vs newborns of adequate weight, stratified in three groups according to their gestational age (GA), as this may reveal potential biomarkers of growth disorders and future metabolic complications.

Methods: 45 NB with IUGR were studied vs. 45 NB of adequate weight as controls. Both groups were divided into 3 subgroups according to the GA (each one with 15 IUGR and 15 controls); Group 1: NB with GA $\geq 29$ and $\leq 32$ weeks. Group 2: NB with $\mathrm{GA} \geq 33$ and

Results: Total serum protein concentration increases according to GA with lower values in cases with IUGR, with significant differences in groups 2 and 3 vs normal NB. There is also a progressive increase along the extraction timepoints, with the maximum at 7-10 days in normal NB, with significant differences between both groups.

Conclusion: Serum protein levels in NB with IUGR differ from NB of adequate weight, increasing according to $\mathrm{GA}$ and time of extraction. A research line is initiated with subsequent proteome determination on NB with IUGR vs normal NB to find possible marker targets in the prevention and treatment of later complications.

\section{EPO-193 Perinatal and Neonatal Endocrinology \\ TTF2 gene mutation in dysgenetic congenitally hypothyroid neonates}

Mahin Hashemipour'; Frouzande Mahjoubi'; Ramin Iranpour; Masoud Aminit; Silva Hovsepian ${ }^{3}$

${ }^{1}$ Isfahan University of Medical Sciences, Pediatric Endocrinology,

Isfahan, Islamic Republic of Iran; '2Iran Blood Transfusion Organization

Research Center (IBTO), Department of Cytogenetics, Tehran, Islamic Republic of Iran; ${ }^{3}$ Isfahan University of Medical Sciences, Child Health Promotion Research Center, Isfahan, Islamic Republic of Iran; ${ }^{4}$ Isfahan University of Medical Sciences, Endocrine \& Metabolism Research Center, Isfahan, Islamic Republic of Iran

Background: Thyroid transcription factor 2 (TTF2) or Forkhead Box E1 (FOXE1) is a polyalanine domain proteins that have an important role in the morphogenesis and development of thyroid gland. Mutations of TTF2 gene have been identified in dysgenetic congenitally hypothyroid neonates.

Objective and hypotheses: Considering the high rate of congenital hypothyroidism in our region, in this study, the mutations of TTF2 gene were studied among these patients.

Methods: In this study the entire region of TTF2 gene of 50 patients with congenital hypothyroidism due to thyroid dysgenesis referred to Isfahan Endocrine and Metabolism Research center was studied by direct DNA sequencing. The mutations were studied after amplification of TTF2 gene by PCR method. Mutations of each exon of TTF2 gene were performed by adjacent primers of the whole encoding region.

Results: We did not find any mutation in TTF2 gene. Three was a serotonin polymorphism among $74 \%$ of studied patients. The length of TTF2 polyalanine tract was 14 aminoacids in most of the patients.

Conclusions: We reveal no mutation in TTF2 in our $\mathrm{CH}$ dysgenetic patients. According to literature review, reported cases of TTF2 gene mutations among dysgenetic $\mathrm{CH}$ patients are rare. For more conclusive results we recommended, further studies with large sample size and investigation of other genes involved in thyroid development.

In Addition, genetic susceptibility to TD may lack the simple Mendelian pattern of inheritance as pointed by Castanet et al.Also, these observations can power the role of epigenetic modifications in TD, suggested by Tonacchera et al. which need to be investigated also. The findings of this study regarding the length of TTF2 polyalanine tract indicated the possible correlation between TTF2 polyalanine tract length polymorphism and genetic susceptibility to thyroid dysgenesis among patients with congenital hypothyroidism.

\section{EPO-194 Perinatal and Neonatal Endocrinology \\ Experience in the use of growth hormone in Noonan syndrome}

Nellya Koroleva

Oblastnaya detskaya klinicheskaya bolnitsa, Department of

Endocrinology, Yekaterinburg, Russian Federation

Background: Noonan syndrome is a rare congenital disorder. It is inherited in an autosomal dominant pattern. It runs in families, but also occurs sporadically. The phenotype bears similarities to that of Turner syndrome, however, Noonan syndrome occurs in both males and females with a normal genotype. The syndrome occurs in about 1 in 8000 newborns and in both sexes.

Objective and hypotheses: Noonan syndrome is characterized by growth retardation syndromic. The perspective is to evaluate the effectiveness of a symptomatic treatment for this pathology of growth hormone

Methods: Patient G. was born at term 35 weeks with a height of $52 \mathrm{~cm}$ and a weight of $3 \mathrm{~kg}$; diagnosed: congenital heart disease: pulmonary stenosis, patent ductus arteriosus, congenital hydronephrosis of the kidneys, corneal opacity, astigmatism, pigeon breast, plano-valgus deformity of the foot. A failure to thrive is noted in the first months of life; at age 4 years with a height of $86 \mathrm{~cm}$ and a weight of $11.2 \mathrm{~kg}$. The patient diagnosed with Noonan syndrome. Patient G. was observed and treated by standard schemes without significant dynamics over the next 2 years; at age 6 years - a height of $100 \mathrm{~cm}$ and a weight of $12.1 \mathrm{~kg}$. Additionally, at age 6 years the $\mathrm{GH}$ was designated (Rastan, production Pharmstandard, Russia) at a dose of $0.6 \mathrm{mg} /$ day $(0.05$ $\mathrm{mg} / \mathrm{kg} /$ day).

Results: By 7 years of age - a height of $114 \mathrm{~cm}$ and a weight of $19 \mathrm{~kg}$. In the next two years the child received GH and GH dose corrected in accordance with the increase in body weight. By 10 years of age the patient G. has reached a height of $135 \mathrm{~cm}$ and a weight of $26 \mathrm{~kg}$ against $\mathrm{GH}$ replacement therapy at a dose of $1.3 \mathrm{mg} /$ day. The diagnosis of concomitant heart disease, hydronephrosis of the kidneys is completely removed. The child studies at specialized school, also attends a music school, is fully socialized.

Conclusions: The designation of GH improves physical health and quality of life for patients with Noonan syndrome.

\section{EPO-195 Perinatal and Neonatal Endocrinology \\ Familial panhypopituitarism: is neonatal hormone testing necessary?}

Chiara Maria Damia; Giuseppe Cannalire; Maria Piera Ferrarello; Alessandra Musio; Gisella Garbetta; Gabriella Pozzobon; Berardo Di Natale; Giuseppe Chiumello

University Vita-Salute S. Raffaele, Department of Pediatrics, Endocrine Unit, Milan, Italy

Background: Neonatal panhypopituitarism (MPHD) is a rare disorder (incidence of 1-2:100.000 live newborns), occasionally familial. Several studies have demonstrated a higher frequency of Magnetic Resonance (MR) abnormalities in children with MPHD including a small to absent anterior pituitary gland, a small or truncated stalk, and an ectopic posterior pituitary (EPP). Objective and hypotheses: To determine the utility of neonatal hormone testing in familial MPHD.

Methods: A female infant weighing $2.040 \mathrm{~kg}$ (25th-50th percentile) was born at $34+1$ weeks of gestation by caesarean section performed for breech presentation. Her head circumference was $31 \mathrm{~cm}(25$ th -50 th percentile) and length was $42 \mathrm{~cm}$ (10th percentile). On examination, she was noted to have hexadactyly of the hands, syndactyly of the feet and choanal stenosis. During her first days of life she showed low blood glucose and sodium values requiring intravenous fluid administration. She developed prolonged jaundice requiring phototherapy for 10 days. A family history of MPHD (father: GH deficiency, TSH deficiency, small anterior pituitary gland and EPP; uncle: GH deficiency and a small anterior pituitary gland) and of hexadactyly was present.

Results: Hormone testing revealed central hypothyroidism (FT3 1,56 pg/ml; FT4 0,48 ng/dl; TSH 3,9 mcU/ml), ACTH deficiency (cortisol 14..44..12..33 $\mathrm{ng} / \mathrm{ml}$; ACTH 7..5..9..6 pg/ml), GH deficiency (basal GH and IGF1 $<2.5$ th percentile). Thyroxine was started at day $12(10 \mathrm{mcg} / \mathrm{kg} / \mathrm{day})$, hydrocortisone was started at day $20(10 \mathrm{mg} / \mathrm{m} 2 /$ day $), \mathrm{GH}$ was started at day $20(0.2 \mathrm{mg} / \mathrm{kg} /$ week). MR showed absent anterior pituitary gland and stalk, and EEP. Genetic analysis (GLI3, PIT-1, PROP1, HESX1, LHX3) showed no abnormalities. Conclusions: In the present report MPHD is familial with variable expressivity and penetrance within the same family. Neonatal hormone testing is recommended in familial MPHD to start therapy as soon as possible preventing life-threatening events. 


\section{EPO-196 Perinatal and Neonatal Endocrinology}

Different evolution in infants with septo-optic dysplasia. Two case reports in comparison Silvana Lauriola; Irene Sibona; Silvia Sordelli; Grazia Morandi; Rossella Gaudino; Franco Antoniazzi

University of Verona, Department of Life and Reproduction, Verona, Italy

Background: Septo-optic dysplasia (SOD) is a rare congenital condition, characterized by classical triade of optic nerve hypoplasia, midline brain defects and pituitary hypoplasia. SOD should be suspected in newborns with hypoglycemia, jaundice, microphallus, nystagmus, associated midline abnormalities. Risk factors are young and primigravida mothers, antenatal smoke alcohol and drugs, infections, maternal diabetes poorly controlled.

Case reports: BM was born at $39+3 \mathrm{w}$; prenatal history characterized by gestational diabetes (doubtful consumption of insulin), IUGR, maternal smoke and mild psychiatric disorders. Prenatal ultrasound showed hydrocephalus, ventricular enlargement and agenesis of corpus callosum. Cerebral MRI confirmed brain malformation compatible with SOD. She never showed until now any sign of hypopituitarism or visual impairment, just mild development delay and hearing impairment. She's followed by neurologists and endocrinologists. RV was born at $40+6 \mathrm{w}$; insulin-dependent gestational diabetes (doubtful consumption of insulin). Prenatal diagnosis with MRI of holoprosencephaly with SOD. At birth onset of clonie with generalized hypotonia. Brain MRI confirmed malformation compatible with SOD. Further tests documented: optic nerves hypoplasia with almost complete blindness; micropenis, arched palate; panhypopituitarism and frequent hypoglycemiae; he started replacement therapy. He recured frequently in the first year of life in the emergency room for fever often being critical. He developed diabetes insipidus, so he begun therapy with nasal vasopressin subsequently suspended for continuing episodes of fluctuating weight gain and restriction of diuresis. Afterward he was on good salt and water balance and stability of paroxysmal crises. The child was found dead while he was in a time of relative prosperity. Conclusions: We report two SOD cases with similar background (gestational diabetes poorly controlled) and different evolution. We underline the necessity of a strict follow-up in this rare condition.

\section{EPO-197 Pituitary}

\section{Pituitary function in children following infectious diseases of the central nervous system}

Yael Levy-Shraga; Inbal Gazit; Dalit Modan-Moses; Orit Pinhas-Hamiel Safra Children's Hospital, Sheba Medical Center, Pediatric Endocrine and Diabetes Unit, Ramat-Gan, Israel

Background: Recent studies in adult patients found that isolated or combined pituitary deficiencies can develop in a considerable proportion of patients after acute infectious meningitis.

Objective and hypotheses: The aim of the study was to evaluate pituitary function of children with previous meningitis/encephalitis.

Methods: 79 previously healthy children ages 0-16 years were admitted to our hospital due to meningitis/encephalitis between 2007 and 2010. The diagnosis of the infectious disease was proven by clinical findings, cerebrospinal fluid examination and culture. 25 families were lost for follow-up, 54 were interviewed by phone and 14 agreed to participate in the study. The evaluation included physical examination, auxologic parameters as well as measuring basal levels of TSH, free T4, cortisol and IGF1.

Results: The study cohort composed 9 males and 5 females. Their mean age at time of infectious meningitis was 3.8 \pm 5.4 (range 0.03-15.8). Their mean age at clinical exam was $6.4 \pm 6.4$ years (range $1.2-20$ years). The interval time between the acute event and clinical evaluation was $2.7 \pm 1.2$ years $(0.7-4.3)$. Mean height percentiles was $42.6 \pm 28.5$, (range 1.5-97). Comparing the difference between patients' height z-score and their genetic target height z-score showed that 3 patients were more than 1 standard deviation below their target height. These patients require further follow up and evaluation. Thyroid tests and basal cortisol levels were normal for all children.

Conclusions: Children with previous CNS infection failing to grow on their genetic target percentiles require endocrine evaluation.

\section{EPO-198 Pituitary}

Partial hypopituitarism in a patient with Sturge Weber syndrome

Sunil Kumar Kota'; Siva krishna Kota²; Lalit Kumar Meher'; SVS Krishna'; Kirtikumar D Modi

${ }^{1}$ Medwin Hospital, Endocrinology, Hyderabad, India; ${ }^{2}$ Central Security Hopsital, Anesthesia, Riyadh, Saudi Arabia; ${ }^{3}$ MKCG medical College, Medicine, Berhampur, India

Background: Growth hormone deficiency and central hypothyroidism has been previously reported to occur in sturge weber syndrome (SWS)

Objective: We present a known patient of SWS coming to fore with partial hypopituitarism.

Methods: Clinical, laboratory, and imaging data are reported on a known case of SWS who was referred for evaluation of short stature

Result: A 16 year old male diagnosed to have SWS was evaluated of short stature. There was port wine stain (PWS) involving left half of face extending variably to right half, with hyperplastic gum, lips and nonblanchable erythema extending to left upper limb. With $<3$ rd percentile height $(155 \mathrm{~cm}$, height age- 13 years and height SDS -2.8) for age and Tanner's puberty stage I (testes bilateral $3 \mathrm{ml}$ ), dry skin and delayed relaxation of ankle reflex, his laboratory parameters are mentioned in table 1 . Computed tomography of the brain revealed serpiginous gyriform cortical calcification with atrophic right atmosphere. Contrast study revealed choroid plexus hypertrophy and leptomeningeal enhancement. After confirming cortisol sufficiency (stimulated peak serum cortisol after $5 \mathrm{U}$ insulin was $24 \mu \mathrm{g} / \mathrm{dl}$ at blood glucose nadir of $35 \mathrm{mg} / \mathrm{dl})$, patient was prescribed oral thyroxine replacement $(50 \mu \mathrm{g})$, testosterone enanthate $250 \mathrm{mg}$ intramuscularly every 3 weekly. After achievement of euthyroidism, growth hormone stimulation test with insulin $(0.1 \mathrm{U} / \mathrm{kg}$, i.e. $5 \mathrm{U})$ and clonidine $(0.15 \mathrm{mg} / \mathrm{m} 2)$ revealed growth hormone deficiency $(4 \mathrm{ng}$ $\mathrm{ml}$ at blood glucose nadir of $38 \mathrm{mg} / \mathrm{dl}$ with insulin and $3.4 \mathrm{ng} / \mathrm{ml}$ with clonidine respectively). The patient was simultaneously prescribed recombinant growth hormone $0.3 \mathrm{mg} / \mathrm{kg}$ / week in 7 divided subcutaneously daily at night. In view of the combined picture of central hypothyroidism, hypogonadotropic hypogonadism, growth hormone deficiency in presence of an organic brain lesion, the diagnosis of partial hypopituitarism was established.

Conclusion: Hypothalamic-pituitary axis is at risk for structural/ functional impairment in patients with Sturge Weber Syndrome.

$\begin{array}{lcc}\text { Parameters } & \begin{array}{c}\text { Normal } \\ \text { values }\end{array} & \begin{array}{c}\text { Patient's } \\ \text { values }\end{array} \\ \text { T4 }(\mu \mathrm{g} / \mathrm{dl}) & 5-12 & 3.1 \\ \text { TSH }(\mu \mathrm{lU} / \mathrm{l}) & 0.45-5.0 & 1.2 \\ \text { Testosterone } 8.00 \mathrm{AM}(\mathrm{ng} / \mathrm{ml}) & 2.9-15.0 & 1.6 \\ \text { FSH }(\mathrm{IU} / \mathrm{l}) & 1.4-18.1 & <0.3 \\ \mathrm{LH}(\mathrm{IU} / \mathrm{l}) & 1.5-9.3 & <0.3 \\ \text { Cortisol } 8.00 \mathrm{AM}(\mu \mathrm{g} / \mathrm{dl}) \text { Cortisol 8.00 AM }(\mu \mathrm{g} / \mathrm{dl}) & 5-20 & 12 \\ \text { ACTH }(\mathrm{pg} / \mathrm{ml}) & 10-46 & 30 \\ \text { Prolactin }(\mathrm{ng} / \mathrm{ml}) & 1.6-18.7 & 24.8 \\ \text { GH }(\mathrm{ng} / \mathrm{ml}) & - & 0.4\end{array}$

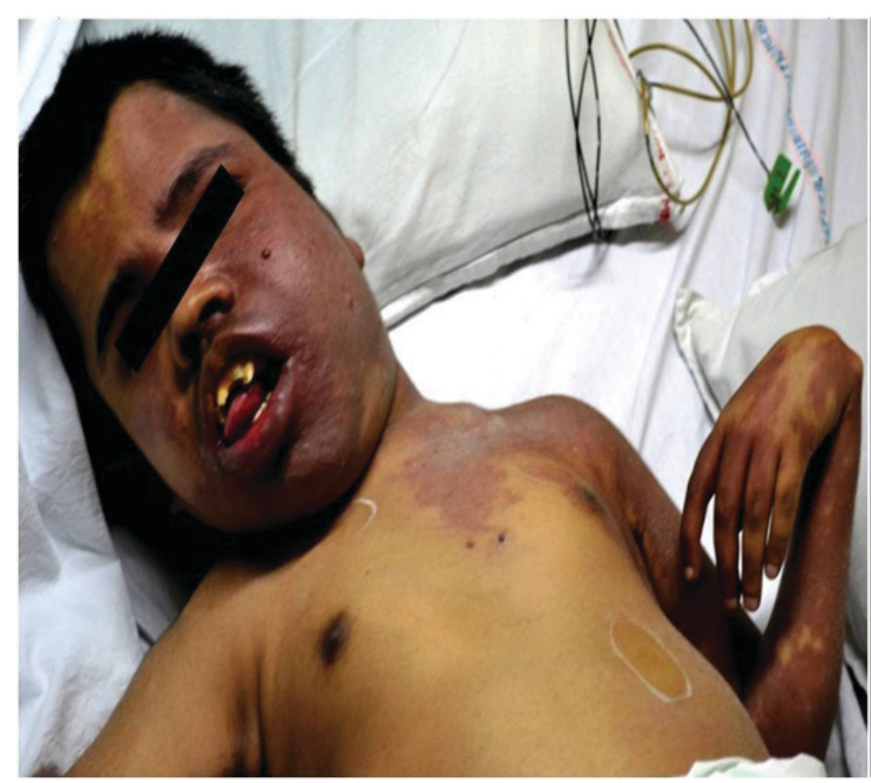




\section{EPO-199 Pituitary}

Syndrome of adipsic diabetes insipidus- report

of six paediatric cases

Dominika Janus; Malgorzata Wojcik; Agata Zygmunt-Gorska; Jerzy Starzyk

Polish-American Children's Hospital, Department of Paediatric and

Adolescent Endocrinology, Chair of Paediatrics, Krakow, Poland

Background: Adipsic diabetes insipidus (DI) is a syndrome which can be observed among patients with various congenital or acquired diseases of the brain.

Objective and hypotheses: Presentation of symptoms, complications and proposition of management protocol in children diagnosed with adipsic DI. Methods: Clinical and biochemical analysis of six paediatric patients diagnosed with adipsic DI, four boys aged 5, 13, 16, and 17 years and two girls aged 2.5 and 13 years.

Results: Two patients were diagnosed with germinoma, two with astrocytoma, and two with idiopathic central DI. Two patients had severely impaired vision and one had unilateral paralysis. In all patients loss of the thirst reflex was observed, due to surgical, tumoral or probably congenital lesion of the lamina terminalis, where the osmoreceptor neurons are located. All had absence of the voluntary ingestion of liquids. The serum electrolytes in all patients showed serum sodium concentration from 157 to $176.6 \mathrm{mmol} / \mathrm{l}$ with plasma osmolality from above $300 \mathrm{mOsm} / \mathrm{kg}$. The supervised by medical staff and parents and calculated based on body weight and slowly increased oral water intake together with DDAVP supplementation enabled rehydration, restored the plasma osmolality and the normal levels of serum sodium. Poliuria was absent in three most severely dehydrated patients on admission (however it was noted shortly after the neurosurgery) and it appeared after rehydration. In two patients in whom DDAVP therapy was withdrawn based on lack of poliuria deep venous thrombosis developed.

Conclusions: The fluid intake in patients diagnosed with adipsic DI need to be supervised daily based on calculated constant volume of oral fluids, daily measurements of fluid balance, body weight and sodium levels, especially in patients whose vision is compromised or who are physically unable to take care of themselves. Lack of fluid intake supervision may lead to severe complications of chronic hyperosmolar status.

\section{EPO-200 Pituitary \\ Prognosis of children with polyuria: a retrospective study of 54 children}

Yasunori Wada'; Terutaka Tajima ${ }^{1}$; Masako Izawa²; Shigehiro Enkai; Keiko Aso ; Junko Igaki'; Daisuke Ariyasu'; Masahiro Goto'; Yukihiro Hasegawa'

'Tokyo Metropolitan Children's Medical Center, Endocrinology and Metabolism, Tokyo, Japan; ${ }^{2}$ Aichi Children's Health and Medical

Center, Endocrinology and Metabolism, Aichi, Japan; ${ }^{3} \mathrm{Fussa}$ hospital,

Pediatrics, Tokyo, Japan; ${ }^{4}$ Toho University Omori Medical Center,

Pediatrics, Tokyo, Japan

Background: Polyuria has generally been defined as a urine output exceeding $2 \mathrm{~L} / \mathrm{sqm}$ in children. Central diabetes insipidus (CDI), Nephrogenic diabetes insipidus (NDI) and primary polydipsia are three major causes of this symptom. However, epidemiological studies focusing on prognosis have not been reported.

Objective and hypotheses: To present fatal episodes in a long-term follow up of children with polyuria.

Methods: A retrospective study of 54 children (median age at diagnosis: 5.8 years, follow-up period: one to 12 years) with polyuria in our institution between 2,000 and 2,012 was done. Children with diabetic ketoacidosis were excluded. Clinical courses of fatal episodes were analyzed.

Results: The clinical courses of fatal episodes were classified into 1) water intoxication and 2) adrenal failure/hypoglycemia as stated below. 1) Water intoxication was observed in children with primary polydipsia $(n=2)$, Munchhausen syndrome by proxy $(n=1)$, NDI $(n=1)$ and CDI $(n=1)$. The episodes of primary polydipsia were related to misdiagnosis, which was based on inadequate water restriction test. DDAVP was used after misdiagnosis, resulting in water intoxication. In Munchhausen syndrome by proxy, his mother abused DDAVP, which was prescribed for nocturia. In NDI, rapid water intake (1 L for a few hours) by patient himself was the cause. The reason in CDI was intravascular water refilling from the third space after surgery of cleft palate and lip, which has not been described as a cause of water intoxication. 2) Adrenal failure/hypoglycemia was observed in Septo-optic dysplasia $(n=1)$ and CDI with hypopituitarism $(n=3)$. The episodes of Septo-optic dysplasia were frequent hypoglycemic seizure even though adequate amount of hydrocortisone had been taken, suggesting the importance of prevention of hypoglycemia. Two of the three CDI children deceased, probably due to crisis of adrenal failure.

Conclusions: The risks of water intoxication and adrenal failure/ hypoglycemia as observed in our study should be recognized.

\section{EPO-201 Pituitary \\ ACTH-secreting pituitary tumor in 14-year-old boy- case report \\ Beata Sawicka; Joanna Towinska; Edyta Pietrewicz; \\ Hanna Borysewicz-Sanczyk; Artur Bossowski \\ Medical University in Bialystok, Department of Pediatrics, \\ Endocrinology, Diabetology with Cardiology Division, Bialystok, Poland}

Background: Cushing's syndrome is a nonspecific name for any source of excessive glucocorticoids. There are four main causes: exogenous glucocorticoids (ACTH-independent), pituitary Cushing's syndrome (ACTH-dependent), ectopic production of $\mathrm{ACTH}$ (ACTH-dependent) and adrenal tumors (ACTH-independent).

Of the endogenous causes of Cushing's syndrome, pituitary Cushing's disease accounts for at least $70 \%$ of cases.

Objective and hypotheses: The female-to-male incidence ratio is approximately 5:1 for Cushing's syndrome due to an adrenal or pituitary tumor. The clinical manifestations of Cushing's syndrome can be subtle or nonspecific. Patients with pituitary tumors causing Cushing's syndrome should undergo pituitary surgery by an experienced neurosurgeon because none of the other treatment options are ideal.

Method: We present a case of 14-year-old boy, diagnosed with obesity, who had incorrect serum cortisol and ACTH levels and an elevated 24-hour urinary level of free-cortisol.The height (3-10 centile) and weight (90-97 centile)were measured during the physical examination. Our patient had not any other clinical symptoms of excessive levels of serum cortisol.

Results: For the purpose of screening test for Cushing's syndrome we did the low-dose dexamethasone suppression test,which the serum cortisol levels were not suppressed after overnight 1-mg dexamethasone administration. We diagnosed ACTH-dependent Cushing's syndrome. When the pituitary MRI in the patient was normal,the elevations of ACTH levels suggested ectopic secretion of ACTH. A chest radiograph was negative.

During octreotide scintigraphy, which may be helpful in detecting ectopic ACTH tumors because some neuroendocrine tumors typically have cell surface receptors for somatostatin, we observed more accumulated marker in the pituitary gland.

In control a contrast- enhanced high-resolution magnetic resonance imaging (MRI) we found a pituitary tumor,like an ACTH- secreting microadenoma, which surgical removal by an expert pituitary surgeon was planned.

Conclusions: ACTH- dependent Cushing's syndrome is a rare caused by pituitary microadenoma.

\section{EP0-202 Pituitary \\ Oral desmopressin lyophilisate (MELT) for the treatment of central diabetes insipidus in very young infants: experience in two cases Kathleen De Waele ${ }^{1}$; Ann De Guchtenaere ${ }^{2}$; Johan Vande Walle; Kris De Coen ${ }^{3}$; Ann Raes'; Sara Van Aken ${ }^{1}$; Martine Cools'; Jean De Schepper \\ ${ }^{1}$ Ghent University Hospital, Department of Pediatric Endocrinology, Ghent, Belgium; ${ }^{2}$ Ghent University Hospital, Department of Pediatric Nefrology, Ghent, Belgium; ${ }^{3}$ Ghent University Hospital, Department of Neonatology, Ghent, Belgium}

Background: Central diabetes insipidus (CDI) is a very rare entity in (premature) neonates and infants. Early recognition and treatment with desmopressin (oral, intranasal or subcutaneous) are important to prevent severe morbidity and mortality. However, because of the need for subtle dose adjustments and high variability in absorption of oral and intranasal formulations, infants are at greater risk for hyponatremia and cerebral oedema than older children.

Objective: To report our experience with oral desmopressin lyophilisate 
(Minirin MELT ${ }^{\circledR)}$ ) in two infants (one premature of 28 weeks), who presented with $\mathrm{CDI}$ at the age of 12 and 62 days respectively.

Methods: They were started on $15 \mu \mathrm{g}$ and dosing was adjusted over the following weeks up to 45 to $60 \mu \mathrm{g}$ daily with close monitoring of diuresis, sodium levels and weight. Caregivers were instructed to monitor weight, to weigh all diapers and to administer Minirin Melt ${ }^{\circledR}$ as soon as a normal diuresis for age was reached since the last dose, usually once per 24 hours.

Results: This regimen resulted in stable random sodium concentrations without severe hypo- or hypernatremia. Both infants are gaining weight properly thus far during their first years of life.

Conclusion: Treatment of CDI is primarily aimed at decreasing urine output. As infants consume almost all their calories in liquid form, they have an obligatory high urine output. They are prone to water intoxication if fixed antidiuresis is achieved using long acting desmopressin. In our experience in these two cases, sublingually administered Minirin Melt@ together with close follow up and parent education can be successfully used in infants with CDI and might result in more stable serum sodium concentrations as compared to

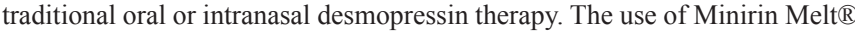
in infants with CDI appears to be effective, easy to use, and well tolerated.

\section{EPO-203 Pituitary}

\section{Central diabetes insipidus in a $\mathbf{3 . 5}$ month old male infant}

Nevenka Slaveska'; Marina Krstevska-Konstantinova ${ }^{2}$

${ }^{1}$ Pediatric Clinic, Metabolism, Skopje, Macedonia, Fyrom; ${ }^{2}$ Peditric

Clinic, Endocrinology and Genetics, Skopje, Macedonia, Fyrom

Background: Central diabetes insipidus (CDI) is a rare disease in children, especially extremely rare in infants. The signs and symptoms depend on etiology and age at presentation. The main features of CDI are great water loses, presence of high plasma osmolality, low osmolality of urine and failure to trieve.

Objective and hypotheses: We describe a 3,5 month old male infant, who suffered from polyuria and polydipsia for several weeks.

Results: At admission the performed investigations revealed: Posm - 263 $\mathrm{mOsm} / \mathrm{kg}$, serum Na $-143 \mathrm{mmol} / 1, \mathrm{~K}-4.4 \mathrm{mmol} / \mathrm{l}, \mathrm{Ca}-2.29 \mathrm{mmol} / \mathrm{l}$, Uosm$56,2 \mathrm{mOsm} / \mathrm{kg}$, Usp.gr.- 1000. There was a dramatic response after vasopressin test: Posm - $259 \mathrm{mOsm} / \mathrm{kg}$, serum Na - $135 \mathrm{mmol} / \mathrm{l}, \mathrm{K}-4.3 \mathrm{mmol} / \mathrm{l}$, Ca- $2.2 \mathrm{mmol} / \mathrm{l}$, Uosm - $385 \mathrm{mOsm} / \mathrm{kg}$, Usp.gr. - 1015. CNS sonography was normal. MRI -sella turcica showed hypoplastic pituitary gland. T4 - 11.8 $\mu \mathrm{gr} / \mathrm{dl}, \mathrm{TSH}-1.48 \mathrm{mU} / \mathrm{ml}$,Cortisol $21.9 \mu \mathrm{g} / \mathrm{l}$-all in normal range. The results confirmed the diagnosis of CDI.

Conclusions: The patient was discharged in a well condition, and enough fluid intake was recommended. Now, he is 11 months old infant, his gain weight is normal, as well as his psychomotor development. Congenital abnormality of the pituitary gland contributed to the development of central diabetes insipidus in our patient.

\section{EPO-204 Pituitary \\ Precocious puberty in a 2 years old girl with a pituitary microadenoma \\ Carmen Temboury \\ Hospital Ntra. Sra. America, Paediatric Endocrinology, Madrid, Spain}

Background: Pituitary tumors are rare in chilhood (1 per million children). Most benign and secreting hormones: $\mathrm{ACTH}, \mathrm{GH}$, prolactin, TSH, and unusually gonadotropins. Often are manifestation of genetic conditions.

Objective: Report clinical, laboratory and magnetic resonance imaging (MRI) of a 2 years old girl with precocious puberty and pituitary microadenoma. Case report: A 2-yr-old girl with pubic hair, breast development, maturation of external genitalia (Tanner stage III) and accelerated linear grow beginning 6 months before presentation. Weight $13 \mathrm{~kg}$ (p75), height 92 cm (p97) Family history negative. TSH $3.7 \mathrm{microIU} / \mathrm{mL}$ and FT4 $1.55 \mathrm{ng} / \mathrm{dL}, 17 \mathrm{OH}$ progesterone $1.6 \mathrm{ng} / \mathrm{ml}$, prolactin $29.6 \mathrm{ng} / \mathrm{mL}$, testosterone $0.1 \mathrm{ng} / \mathrm{ml}$, cortisol $20 \mathrm{mcg} / \mathrm{dL}, 17$ beta estradiol $71 \mathrm{pg} / \mathrm{mL}$. ACTH: $22 \mathrm{pg} / \mathrm{ml}$, GH $0.18 \mathrm{ng} /$ $\mathrm{ml}$, IGF1 $73.5 \mathrm{ng} / \mathrm{ml}$, IGFBP3 $2.4 \mathrm{mcg} / \mathrm{mL}$, normal osmolality, urinary free cortisol: $350 \mathrm{mcg} / 24 \mathrm{~h}$, beta $\mathrm{HCG}<5 \mathrm{mIU} / \mathrm{mL}$, alpha fetoprotein $1.5 \mathrm{IU} / \mathrm{mL}$. LH-RH stimulation test: LH 5.7, peak 95 and FSH 5.1, peak $23.4 \mathrm{mcUI} / \mathrm{mL}$. Bone age $4.0 \mathrm{yr}$ at $2.0 \mathrm{yr}$ old. Normal abdominal and pubertal pelvic sonography. CNS MRI: pituitary microadenoma of $5 \mathrm{~mm}$ diameter in rigth lobe, adenohypophysis of normal size, no others alterations
Results: Central precocious puberty was treated with Triptorelin $(100 \mathrm{mcg}$ $\mathrm{kg}$ ) every 28 days. After 1 year free of neurological symptoms, grow rates normalize and breast development regress. At 3rd yr: weight $17 \mathrm{~kg}$, height $100 \mathrm{~cm}$, height velocity $7.5 \mathrm{~cm} / \mathrm{yr}$, Tanner stage I. FSH $2.4 \mathrm{mUI} / \mathrm{ml}$, LH $1.46 \mathrm{mUI} / \mathrm{ml}$; estradiol $27.2 \mathrm{pg} / \mathrm{mL}$, prolactin $11.8 \mathrm{ng} / \mathrm{ml}$; GH $0.18 \mathrm{mcg} / \mathrm{dL}$, IGF1 $166 \mathrm{ng} / \mathrm{mL}$; IGFBP3 $3.7 \mathrm{mcg} / \mathrm{dL}$; TSH $7.28 \mathrm{mcUI} / \mathrm{ml}$, FT4 $1.09 \mathrm{ng} / \mathrm{dL} ;$ ACTH $22.9 \mathrm{pg} / \mathrm{ml}$, cortisol $11.7 \mathrm{mcg} / \mathrm{mL}$. Sonography: normal ovarian and uterine size. CNS MRI unchanged.

Conclusions: Despite the rarity of a gonadotropin-secreting pituitary adenoma in childhood, no other cause for the early puberty was found. Good clinical response to LH-RH analogues, incomplete suppression of LH, FSH and estradiol, slight increase of TSH and unchanged RMI adenoma after one year was observed.

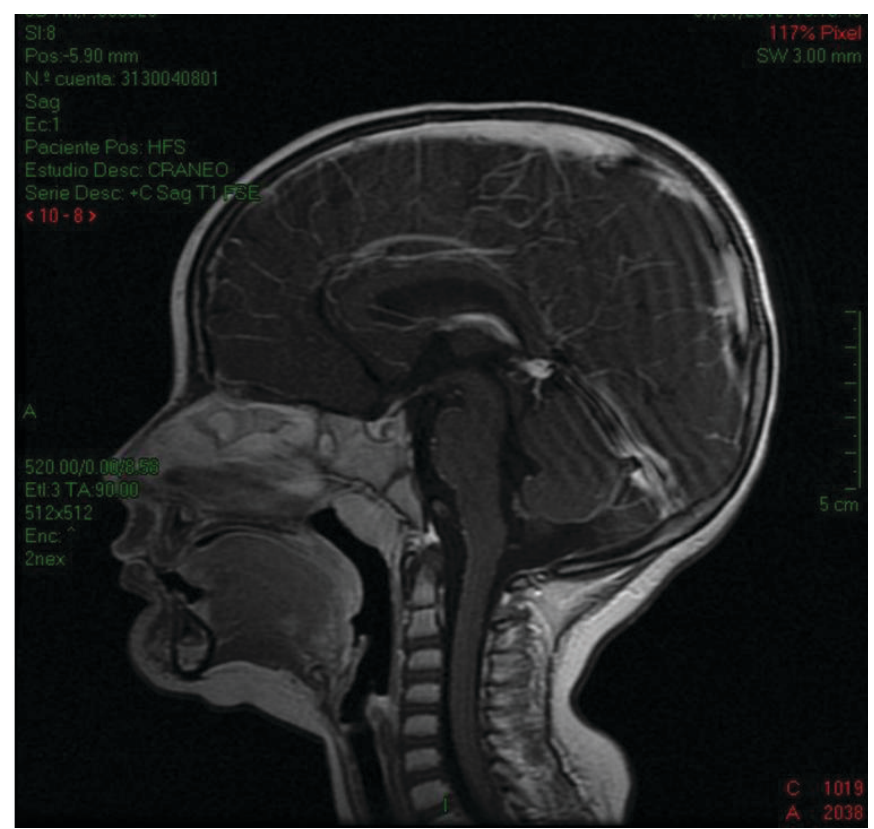

\section{EPO-205 Pituitary}

\section{Study on sex hormones and gonadotropin- releasing hormone stimulation test among simple premature thelarche, precocious puberty and gonadotropin independent precocious puberty in female children less than four years old \\ Shujuan Guo \\ University of Medical, the Children's Hospital, Endocrinology, Chongqing, China}

Background: The majority of precocious puberty in female children less than four years old is simple premature thelarche.

Objective and hypotheses: To understand the secretion characteristics of hypothalamic-pituitary-gonadal axis in simple premature thelarche, and to distinguish from central precocious puberty and gonadotropin independent precocious puberty.

Methods: Compare the foundation levels of sex hormones (FSH, LH, PRL, E2) and gonadotropin-releasing hormone stimulation test among simple premature thelarche (374 cases), central precocious puberty ( 23 cases) and gonadotropin independent precocious puberty ( 99 cases); 374 cases were classified six groups according to age,and compared the indexs above mentioned . Results: 1.The peak amplitude of FSH in simple premature thelarche group was significantly higher than in central precocious puberty group $(\mathrm{P}<0.0001)$, but the foundation level and the peak amplitude of LH, the ratio of peak amplitudes of LH and FSH in central precocious puberty group was significantly higher than in simple premature thelarche group $(\mathrm{P}<0.0001)$, the foundation level of FSH had no statistically significant differences between the two groups $(\mathrm{P}>0.05)$; the foundation level and the peak amplitude of FSH and $\mathrm{LH}$ in gonadotropin independent precocious puberty group was lowest $(\mathrm{P}<0.0001)$. 
2.The basal level of FSH in simple premature thelarche group (mean value $4.68 \pm 2.32 \mathrm{mIU} / \mathrm{ml})$ was significantly higher than $\mathrm{LH}(0.20 \pm 0.09 \mathrm{mIU} /$ $\mathrm{ml}$ ), The higher levels of FSH and LH respectively appeared in 7 months to 1.5 ages and 7 months to 12 months.

In the gonadotropin -releasing hormone agonist stimulation test, the peak amplitude of FSH (mean value $34.82 \pm 12.28 \mathrm{mIU} / \mathrm{ml}$ )was significantly higher than $\mathrm{LH}(6.71 \pm 2.93 \mathrm{mIU} / \mathrm{ml})$, the ratio of the peak amplitudes of LH and FSH, there had no statistically signifant differences among the six groups $(\mathrm{P}>0.05)$. Conclusions: The sex hormones of simple premature thelarche had secretion tendency; the sex hormones and gonadotropin -releasing hormone stimulation test were differences among the three precocious puberty.

\section{EPO-206 Pituitary \\ Hyperprolactinemia in children: aetiology, clinical aspects and treatment}

Gonul Catli'; Ayhan Abaci'; Ayca Altincik'; Korcan Demir'; ; Sule Can²; Atilla Buyukgebiz ; Ece Bober $^{1}$

${ }^{1}$ Dokuz Eylul University, Pediatric Endocrinology, Izmir, Turkey; ${ }^{2}$ Izmir

Tepecik Training and Research Hospital, Pediatric Endocrinology, Izmir,

Turkey; ${ }^{3}$ Bilim University, Pediatric Endocrinology, Istanbul, Turkey

Background: Hyperprolactinemia is a relatively less frequent disorder in childhood which may present with various signs and symptoms.

Objective and hypotheses: To retrospectively evaluate etiologies and clinical course of hyperprolactinemia in pediatric patients.

Methods: Case records of 11 patients with hyperprolactinemia followed in Dokuz Eylul University and Izmir Tepecik Training and Research Hospital Pediatric Endocrinology Departments were reviewed. Cases with pregnancy, polycystic ovary syndrome, primary hypothyroidism, liver or kidney function disorders and antipsychotic medication were excluded.

Results: The mean age of 11 female patients with hyperprolactinemia at diagnosis was $14.2 \pm 1.3$ years. Microadenomas in 5 patients, macroadenomas in 4 patients and idiopathic hyperprolactinemia (normal pituitary magnetic resonance imaging) in 2 patients were detected.

The most frequent symptoms were menstruel disorders, headache, and galactore. One third of patients had obesity at diagnose. No accompanying anterior pituitary hormone deficiencies were detected. All patients were given bromocriptine as initial therapy. In 2 patients with macroadenoma and in 1 patient with microadenoma, treatment was switched to cabergoline because of resistance and/or incompatibility to bromocriptine. A transsphenoidal surgery was performed for a patient with macroadenoma who had cavernous sinus invasion and visual field defect.

Conclusions: Menstruel disorders, headache and galactore are the most frequent symptoms of childhood hyperprolactinemia. Since surgical therapy usually does not provide cure and recurrence risk is high, dopamine agonist therapy should be the first choice of treatment for microadenoma and macroadenoma patients who does not have neurological findings.

\section{EPO-207 Pituitary \\ Familial neurohypophyseal diabetes insipidus associated with a novel mutation in the arginine vasopressin (AVP) gene \\ Elena Pisareva; Natalya Strebkova; Anatoly Tiulpakov \\ Endocrinology Research Centre, Pediatric Endocrinology, Moscow, Russian Federation}

Background: Familial neurohypophyseal diabetes insipidus (FNDI) is a rare disorder caused by mutations in the arginine vasopressin gene (AVP). There are autosomal dominant and autosomal recessive inheritances.

Objective and hypotheses: We report a family with autosomal dominant neurohypophyseal diabetes insipidus.

Methods: The proband was a 13-year-old girl who developed symptoms of polyuria and polydipsia at the age of 3 . The girl was diagnosed with neurohypophyseal diabetes insipidus at the age of 4 . The diagnosis was established on the basis of clinical symptoms, water deprivation test and adequate response to DDAVP administration. Anterior pituitary hormone levels were normal. The hyperintense posterior pituitary signal was absent on T1-weighted magnetic resonance imaging. The girl received therapy with DDAVP $0,35 \mathrm{mg} /$ day. Four family members in three generations (mother, grandmother and grandgrandfather) also had symptoms of polyuria and polydipsia.
Results: DNA analysis detected a new heterozygous missense mutation p.L73P in the exon 2 of the AVP gene. This mutation is predicted to be "possibly damaging" with a score of 0,458 by the structure-based approach PolyPhen.

Conclusions: We described a novel p.L73P mutation in AVP gene that was associated with familial case of neurohypophyseal diabetes insipidus.

\section{EPO-208 Pituitary \\ Gigantism in a girl with a growth hormone- secreting pituitary adenoma \\ Lavinia La Grasta Sabolic; Marija Pozgaj Sepec; Gordana Stipancic \\ University Hospital "Sestre milosrdnice", Department of Pediatric \\ Endocrinology and Diabetology, Zagreb, Croatia}

Background: Pituitary tumors causing gigantism are rare in childhood and adolescence and are more likely to be invasive or aggressive than those in adulthood.

Objective and hypotheses: We describe a female patient with a growth secreting adenoma of the pituitary gland causing gigantism.

Methods: A case report.

Results: A 14.9 year old girl came to our endocrinology unit because of the primary amenorrhea. She had no history of galactorrhea. On examination her height was $183.3 \mathrm{~cm}, 3.6$ SDS (mid parental height $167 \mathrm{~cm}, 0.8$ SDS) with Tanner stage 5 puberty. Her hands and feet were large with slight swealing, she had a rough voice. The following base-line serum hormone levels were obtained: GH $60 \mathrm{ng} / \mathrm{mL}$, prolactin (PRL) $33.8 \mathrm{ug} / \mathrm{L}$ and IGF-1 $836 \mathrm{ng} / \mathrm{mL}$; kariotype was normal female $(46, \mathrm{XX})$. She had secondary hypothyroidism (TSH $0.3 \mathrm{mIJ} / \mathrm{L}$, fT4 $8.97 \mathrm{pmol} / \mathrm{L}$ ) and adrenal insufficiency (basal morning ACTH $3.7 \mathrm{pmol} / \mathrm{l}$, cortisol $50 \mathrm{nmol} / \mathrm{l}$ ) as well as low basal gonadotrophin levels (LH 0.9, FSH $1.8 \mathrm{U} / 1)$.

A three hour OGTT test revealed normal carbohydrate tolerance but there was no suppression of GH $(36.3-40 \mathrm{ng} / \mathrm{ml})$. MRI of the pituitary gland revealed pituitary macroadenoma with suprasellar extension $(2.7 \times 2.6 \times 3.4 \mathrm{~cm})$. Levotyroxine and hydrocortisone replacement therapy was introduced. She underwent transsphenoidal partial adenomectomy. On immunohistochemical staining cells were GH and PRL positive. One month after the surgery GH remained elevated $(11.9 \mathrm{ng} / \mathrm{mL})$ and repeated OGTT failed to suppress GH concentrations. Postoperative PRL levels were within the normal range. MRI scan showed residuous tumour in the right cavernous sinus. Thus, the treatment with sandostatin analog octreotide (Sandostatin LAR Depot) was started. Gamma knife therapy was planned and performed three months after the initial surgery.

Conclusions: Although rare, a GH secreting pituitary adenoma can be a cause of tall stature in childhood. Thus the early diagnosis is essential in preventing development of macroadenoma with hypopituitarism which requires complex surgical and medicamentous therapy.

\section{EPO-209 Pituitary}

\section{Successful treatment with tolvaptan in a 5-year-old girl with congenital SIADH Hedi Claahsen - van der Grinten'; Floor Neijnens';} Marlies Cornelissen ${ }^{3}$; Ernie Bongers ${ }^{4}$; Annemarie Verrijn Stuart ${ }^{5}$; Stephanie Natsch ${ }^{6}$

${ }^{1}$ Radboud University Nijmegen Medical Centre, Department of Pediatric Endocrinology, Nijmegen, Netherlands; ${ }^{2}$ Rijnstate Medical Centre, Department of Pediatrics, Arnhem, Netherlands; ${ }^{3}$ Radboud University Nijmegen Medical Centre, Department of Pediatric Nephrology, Nijmegen, Netherlands; ${ }^{4}$ Radboud University Nijmegen Medical Centre, Department of Human Genetics, Nijmegen, Netherlands; ${ }^{5}$ University Medical Centre, Department of Pediatric Endocrinology, Utrecht, Netherlands; ${ }^{6}$ Radboud University Nijmegen Medical Centre the Netherlands, Department of Pharmacy, Nijmegen, Netherlands

Background: The syndrome of inappropriate ADH secretion (SIADH) is one of the most common causes of chronic hyponatremia in adults. In these patients selective vasopressin-2-receptor (V2R) antagonists can be used for long term treatment. Recently an oral form, Tolvaptan, was introduced. During childhood, SIADH is mostly transient as after neurosurgery or other neurological conditions. Fluid restriction usually prevents development of acute hyponatriemia. Here, we describe a 5-year-old girl with severe chronic hypo- 
natremia due to SIADH of unknown etiology. She was successfully treated with Tolvaptan.

Case report: The girl was adopted from China at the age of 5 years. She was born with a severe cleft palate but seemed to be otherwise healthy although further information was lacking. One week after arrival to the Netherlands she was admitted to a hospital because of convulsions. Further investigations revealed severe hyponatriemia $(\mathrm{Na} 121 \mathrm{mmol} / \mathrm{l})$. Adrenal insufficiency and renal pathology were ruled out. Fluid restriction up to a daily intake of $75 \mathrm{ml}$ resulted in normalization of serum sodium values. With a daily fluid intake of $500 \mathrm{ml}$ she developed severe hyponatremia $(125 \mathrm{mmol} / \mathrm{l})$ with detectable vasopressin concentrations, high urinary sodium excretion and normal plasma renin levels. Therefore, she was diagnosed with chronic idiopathic SIADH. Because of the low fluid intake resulting in inappropriate feeding and growth we started treatment with oral Tolvaptan $7 \mathrm{mg} /$ day $(0,7 \mathrm{mg} / \mathrm{kg} /$ day $)$. Directly after the first dose, there was a significant decrease in urinary osmolarity with an increase in diuresis resulting in normalization of fluid intake to about 1200 $\mathrm{ml}$ daily with normalizing sodium levels $(133-136 \mathrm{mmol} / \mathrm{l})$. After a treatment period of now 1 year Tolvaptan is well tolerated without detectable side effects.

Conclusion: Tolvaptan is effective in increasing diuresis and normalization of serum sodium in a child with congenital SIADH without detectable side effects.

\section{EPO-210 Pituitary}

\section{ROHHADNET syndrome - case report}

Karolina Kot ${ }^{1}$; Elzbieta Moszczynska ${ }^{1}$; Agnieszka Lecka Ambroziak'; Marek MigdaR; Mieczyslaw Szalecki'

'The Children's Memorial Health Institute, Department of Endocrinology, Warsaw, Poland; ${ }^{2}$ The Children's Memorial Health Institute, Intensive Care Unit, Warsaw, Poland

Background: ROHHAD/ROHHADNET syndrome is a very rare disorder that includes rapid-onset obesity associated with hypoventilation threatening cardiorespiratory arrest, hypothalamic and autonomic disregulation, tumors of neural crest origin.

Objective and hypotheses: We report the first polish case of patient diagnosed with ROHHAD syndrome. 9-year-old boy presented with weight gain, mainly with facial and abdominal distribution, short stature, hyperphagia, hypodipsia, body temperature disregulation (34-39 C degrees), excessive perspiration, cold hands and feet, livedo reticularis and sleep apnea.

Methods: Endocrine and imaging studies were performed. Genetic test to exclude PHOX2B mutation, responsible for central hypoventilation syndrome, remains in course.

Results: Growth hormone $(\mathrm{GH})$ deficiency was confirmed in stimulation tests and treated with GH (improvement of growth rate during 12-month observation). Moderate hyperprolactinemia ( $\max .73,6 \mathrm{ng} / \mathrm{ml}, \mathrm{N}<20$ ) was observed. Thyroid and adrenal axes were normal. Hypernatremia (max.161 mmol/l) without diabetes insipidus was present. Sleep apnea was confirmed by haemoglobin desaturation's episodes at night (min. SatO2 78\%), transcutaneous $\mathrm{PaO} 2$ was $60 \mathrm{mmHg}$, and $\mathrm{PaCO} 2$ was normal. In 72-hour ECG atrio-ventricular dissociation periods were observed. The results indicated the need of ventilation support during the night. Due to elevated concentrations of VMA and epinephrine in urine, MIBG-scintigraphy and $99 \mathrm{mTc}$-tectreotide-scintigraphy were performed and excluded neural crest origin tumor. PRA was found to be considerably high, but aldosterone remained within normal range. Transient hypertension was not confirmed by 24-hour ABPM. CNS MR, thorax and abdomen $\mathrm{CT}$ imaging revealed no abnormalities.

Conclusions: Presented clinical features allow with high probability to diagnose ROHHADNET syndrome in the reported patient. It should be always considered in case of early and rapid-onset obesity with growth failure or other unclear endocrine disturbances.

\section{EPO-211 Pituitary}

Growth hormone deficiency, associated with solitary median maxillary central incisor (SMMCI), skeletal deformities and ocular

\section{anomalies}

Iva Stoeva ${ }^{1}$; Antoaneta Kostova ${ }^{1}$; Savina Agova-Hadjidekova ${ }^{2}$;

Ani Aroyo' ${ }^{1}$; Draga Toncheva ${ }^{2}$

${ }^{1}$ University Pediatric Hospital, Medical University Sofia, Screening and

Functional Endocrine Diagnostics, Sofia, Bulgaria; ${ }^{2}$ Medical University

Sofia, Medical Genetics, Sofia, Bulgaria

Background: SMMCI syndrome (1: 50000 live births) could be associated with septo-optic dysplasia and/or other midline defects.

Objective and hypotheses: SMMCI represents a rare syndrome with wide variability in the phenotypic spectrum.

Methods: 12 yrs old girl, TH- $161.8 \mathrm{~cm}$, short statured maternal relatives. Born from first uneventful pregnancy, 10 days after due date (Caesarian section), BW $2650 \mathrm{~g}$, BL $49 \mathrm{~cm}$ (both <3rd centile). Oxygen supply (airway obstruction) and tube feeding needed during the first days. Congenital bilateral cataract (lense implantation, glasses). Teeth errupted at 4 months: SMMCI. Smaller than her peers since infancy, normal neurodevelopment. Evaluation for growth delay (5 yrs7m): height $100.3 \mathrm{~cm}$ (SDS -2.33), weight $16.5 \mathrm{~kg}$ (SDS -1.2), doll-like face, small nose, protuberant forehead, SMMCI, higharched palate, micrognatia, short neck, acromicria.

Results: GH stimulation tests: exercise (10') GH peak $5.8 \mathrm{mU} / 1$, glucagon (1 $\mathrm{mg} \mathrm{im}$ ) GH peak $22.4 \mathrm{mU} / 1$ (cut-off $26 \mathrm{mU} / \mathrm{l}$ ). TSH $5.02 \mathrm{mU} / 1$, after TRH 30' $34.7,60$ ' 32.5, 90’ $22.5 \mathrm{mU} /$ l-suggestive of hypothalamic defect; MRI: pituitary hypoplasia, interruption of the stalk, normal supratentorial structures. At 7 yrs height $110.7 \mathrm{~cm}$ (SDS - 2.47); BA 6 yrs (G\&P).

Arginine test: GH peak $14.9 \mathrm{mU} / 1$ (partial GHD). Start of rhGH therapy and substantial catch up growth for 3 years. Spontaneous puberty (thelarche) at 9.5 years, fast progression. Spondylolisthesis with fracture of L5 and subsequent orthopedic surgery (11 yrs). Discontinuation of the rhGH and sharp decrease of growth velocity ( $<3$ rd centile).

Present evaluation: scoliosis, height $142.2 \mathrm{~cm}$ (SDS- 2.33), completed puberty, advancing bone age $(+1.5 \mathrm{yr})$.

No association with chromosomal abnormalities as del(18p), $r(18)$, del(7q36qter), 47XXX, del(22q11.2).

Conclusions: The index case contributes to the phenotypic variability in the SMMCI syndrome and still obscure etiology. Lifelong interdisciplinary management in this rare complex disease is needed.

\section{EPO-212 Pituitary \\ Central hypothyroidism (CH), growth hormone deficiency (GHD) and partial cortisol deficiency (PCD) in two Cacauisan sisters with severe short stature (SS)}

\section{Jadranka Popovic}

Children's Hospital of Pittsburgh/UPMC, Division of Endocrinology and Diabetes, Pittsburgh, United States

Background: Multiple pituitary deficiencies are rare particularly when occurring in multiple family members. Several homeodomain transcriptions factors (HTF) have been shown to be involved in human anterior pituitary development and differentiation. Defects in HTF have been associated with various combinations of pituitary hormone deficiencies.

Objective and hypotheses: We present two sisters with severe SS, CH, GHD and PCD.

Methods: Older sister (NM) presented at age 9.8 yrs for evaluation of severe SS. She was otherwise in good general health. Physical exam revealed height $113.1 \mathrm{~cm}$ (SDS:-3.9); weight $21.1 \mathrm{~kg}$ (SDS:-2.6); BMI $16.5 \mathrm{~kg} / \mathrm{m} 2(50 \%)$; Tanner I breast and pubic hair; and otherwise unremarkable. Younger sister (TM) was 8.6 yrs and had severe SS as well. She was otherwise in good general health. Physical exam revealed height $107.3 \mathrm{~cm}$ (SDS:-4.3); weight 17.4 $\mathrm{kg}$ (SDS:-2.1); BMI $17.5 \mathrm{~kg} / \mathrm{m} 2(50 \%)$; Tanner I breast and pubic hair; and otherwise unremarkable. MPH target is $164 \mathrm{~cm}(50 \%)$. FHX is significant for SS in multiple paternal female family members $(147-150 \mathrm{~cm})$. Both parents had a delayed puberty. A $5 \mathrm{y} / \mathrm{o}$ healthy brother is growing on the $75 \%$ (along $\mathrm{MPH})$.

Results: Evaluation showed low free T4 and T4 with inappropriately low TSH. IGF-1 level was low. ACTH test was performed prior to starting thyroid 
replacement therapy. AITT test was performed. Bone age was delayed. MRIs of the brain and pituitary were abnormal (Table 1).

\begin{tabular}{|c|c|c|}
\hline Baseline & NM & TM \\
\hline Chronological Age (years) & 9.8 & 8.6 \\
\hline Height $(\mathrm{cm})$ & 113.1 & 107.3 \\
\hline Height SD & -3.9 & -4.4 \\
\hline Weight (kg) & 21.1 & 17.4 \\
\hline Weight SD & -2.6 & -2.1 \\
\hline $\mathrm{BMI}(\mathrm{kg} / \mathrm{m} 2)$ & 16.5 & 17.5 \\
\hline $\mathrm{BMI} \%$ & 50 & 50 \\
\hline Free T4 (0.8 - $1.80 \mathrm{ng} / \mathrm{dL})$ & 0.47 & 0.26 \\
\hline T4 (6.4 - $13.3 \mathrm{mcg} / \mathrm{dL})$ & 4.0 & 2.1 \\
\hline TSH (0.7-5.7 mlU/mL) & 1.4 & 1.3 \\
\hline IGF-1 (117 "C 771 ng/ml) & $<25$ & $<25$ \\
\hline IGF-BP3 (1.58 - $3.99 \mathrm{mcg} / \mathrm{ml})$ & 1.6 & 1.5 \\
\hline GH-AITT-peak ( $\geq 10$ ng/ml) & 0.20 & 0.26 \\
\hline Cortisol-ACTH-peak ( $\geq 18 \mathrm{mcg} / \mathrm{dl}$ ) & 15 & 15 \\
\hline Karyotype & $46, X X$ & ND \\
\hline Bone age (years) & 7.8 (> 2 SD delayed) & 4.6 ( $>5 \mathrm{SD}$ delayed) \\
\hline
\end{tabular}

L-thyroxine (LTh) and growth hormone (GH) therapy was initiated. Clinical course was significant for improved thyroid and IGF-1 levels, annual growth velocity (GV) and SDS (Table 2)

\begin{tabular}{lcc} 
Rx with GH and L-thyroxine & NM (12 $\mathbf{~ m o})$ & TM (6 mo) \\
\hline Height (cm) & 128.8 & 120.0 \\
Height SD & -2.2 & -2.4 \\
Growth velocity (cm/yr) & 14.3 & 18.8 \\
Weight $(\mathrm{kg})$ & 27.0 & 22.0 \\
Weight SD & -1.9 & -1.9 \\
BMI (kg/m2) & 16 & 15 \\
BMI \% & 30 & 25 \\
Free T4 (0.8 - $1.80 \mathrm{ng} / \mathrm{dL})$ & 0.9 & 1.2 \\
\hline T4 $(6.4-13.3 \mathrm{mcg} / \mathrm{dL})$ & 6.2 & 8.2 \\
IGF-1 (117-771 ng/ml) & 328 & 255
\end{tabular}

Sisters continue to do well on LTh and GH replacements respectively. Cortef was prescribed for stress coverage. Genetic testing for PROP 1 and POU1F1 genes was obtained. Results are pending.

Conclusions: Defects of HTF are rare etiology for multiple pituitary deficiencies. Familial cases of defects in HTF are even rarer. Sisters will require continuous surveillance for the risk of developing new pituitary deficiencies.

\section{EP0-213 Pituitary \\ Congenital sporadic panhypopituitarism and pituitary hypoplasia in a prepubertal boy, without a PROP1-mutation \\ Ani Aroyo ${ }^{1}$; Iva Stoeva' ${ }^{1}$; Shina Pashova'; Sylvia Andonova²; Alexei Savov² \\ ${ }^{1}$ University Pediatric Hospital, Medical University Sofia, Screening and Functional Endocrine Diagnostics, Sofia, Bulgaria; ${ }^{2}$ University Maternity Hospital, Medical University Sofia, National Molecular Genetic Laboratory, Sofia, Bulgaria}

Background: PROP1 mutations are the most common cause of congenital familial combined pituitary hormone deficiencies, especially in patients from Eastern/Central Europe.

Objective and hypotheses: Evolution of panhypopituitarism with early ACTH deficiency in a boy with sporadic GHD due to pituitary hypoplasia, considered to be a candidate for PROP1 gene analysis.

Methods: Born after 3rd uneventful pregnancy, normal delivery, BW 4150g (97centile), BL $52 \mathrm{~cm}$ ( 75 centile), with hypoglycemia (12-hours age, seizures), micropenis, cryptorchidism as neonate; TH-SDS -0.23 . Clinical phenotype at diagnosis: growth retardation (SDS -1.94) as cardinal symptom $(5 \mathrm{yrs} 6 \mathrm{~m})$, associated with truncal obesity, midface hypoplasia, acromicria, dry and yellow skin, BA (G\&P) delay of 3yrs.

Results: Laboratory and clinical signs of central hypothyroidism. Start of
L-T4 treatment $(50 \mu \mathrm{g} / \mathrm{d})$ and evaluation of the stimulated (Arg-HCl) $\mathrm{GH}$ secretion in euthyroid state: severe GHD (GH peak $<3 \mathrm{ng} / \mathrm{ml}$ ), confirmed by the CNS-MRI: anterior pituitary hypoplasia, eutopic neurohypophysis, intact stalk. Institution of rhGH $(0.075 \mathrm{U} / \mathrm{kg} / \mathrm{d})$. During severe infection followed by viral hepatitis, development of manifest secondary adrenocortical insufficiency and need for permanent hydrocortisone substitution (6 yrs of age). The subsequent frequent follow up revealed: a) significant catch up growth (SDSh from -1.94 to 0.13; rhGH0.07-0.09 U/kg/d); b) obesity despite euthyroid state, c) need for close IGF1/IGFBP3 monitoring. The sequencing of PROP1 before puberty start ( $9 \mathrm{yrs} 3 \mathrm{~m})$ could establish only polymorphisms: homozygous in exon $1(59 \mathrm{~A}>\mathrm{G})$ and intron $1(109+3 \mathrm{G}>\mathrm{A})$, heterozygous in exon3 $(424 \mathrm{G}>\mathrm{A})$.

Conclusions: The molecular heterogeneity of congenital hypopituitarism represents still a challenge; the clinical relevance of different polymorphisms remains to be elucidated.

\section{EPO-214 Pituitary \\ Excessive hyperprolactinemia with galactorrhea in 15 year old girl on haemodialysis treated long time with domperidone}

Dimitar Roussinov'; Polina Miteva²; Elissaveta Stefanova

'University Children's Hospital, Endocrinology, Sofia, Bulgaria;

2University Children's Hospital, Haemodialysis, Sofia, Bulgaria

Background: Cronik renal failure / $\mathrm{CRF} /$ and domperidone treatment are known to be associated with elevated values of prolactin. However extremely high levels of prolactine and galactorrhea are very rare.

Objective: 15year old girl with CRF and Haemolytic uremic syndrome on chronic haemodialysis treatment, received domperidone because of severe duodeno - gastric reflux and vomiting. At the end of the 4-th mounth of domperidone treatment galactorrhea was appeared. Prolactin levels was extremely high - $9095 \mathrm{mikroIU} / \mathrm{ml} /$ normal $73-850 \mathrm{mikroIU} / \mathrm{ml} / . \mathrm{MRI}$ of the brain was normal. Domperidone was stopped, galactorrhea disappeared and three mounths latter prolactin was in normal range $-421 \mathrm{mikroIU} / \mathrm{ml}$.

Conclusions: Combination of $\mathrm{CRF}$ and prolonged domperidone treatment may simulate prolactinoma with galactorrhea and excessive high prolactin levels. In our case probably domperidone treatment is cause for hyperprolactinemia and galactorrhea.

\section{EPO-215 Pituitary \\ Multiple pituitary hormone deficiency due to gunshot injury in a 6-year-old girl \\ Huseyin Demirbilek; Mehmet Nuri Ozbek \\ Diyarbakir Children's Diseases Hospital, Pediatric Endocrinology, \\ Diyarbakir, Turkey}

Background: Gunshot injuries of cranial area has an extremely high mortality rate. Herein we present a girl who living with a bullet on the posterior sellar region and causes multiple pituitary hormone deficiency.

Case: A 6-year-old girl was admitted to our clinic with the complaint of headache, polyuria and polydypsia. Her previous history revealed that she was healthy until 5 months ago. In that date, while she was playing in the garden she was injured from her head with a foreign object. Radiological examination revealed a bullet in her skull (Figure). Thus it was thought a gunshot injury. Since the bullet was adjacent to a vital region of brain, she considered inoperable. Thereafter, she was living with a bullet in her brain. Her complaints had started after that. At the time of admission her height was 113.5 cm (25th-50th p), weight was $21 \mathrm{~kg}$ (50th p). Other physical findings were normal. Her 24 hour urine output was $4.5 \mathrm{~L}$. Water deprivation test showed failure to concentrate urine. After a test dose nasal desmopressin $(5 \mathrm{mcg})$ urine output decreased and urine osmolarity increased. She was considered central diabetes insipitus and her complaints were resolved with a maintenance dose of desmopressin. Evaluation of other pituitary hormone revealed central hypothyroidism (free T4:0.5 ng/dl(0.7-1.26) and TSH:1.1 mIU/ml). Low dose ACTH test showed normal adrenal function. Very low growth rate $(0.2 \mathrm{~cm} / 6$ months) during follow up suggested growth hormone(GH) deficiency. Growth hormone stimulation tests for the diagnosis of GH are going on. Now except for mentioned hormonal deficiencies and a slight headache she has not suffering from her bullet in here brain. 
Conclusion: Present case was found interesting in here outcome of gunshot injury which causes pituitary hormone deficiency. However, although she had a life threatening injury to be alive still may be a chance for she.

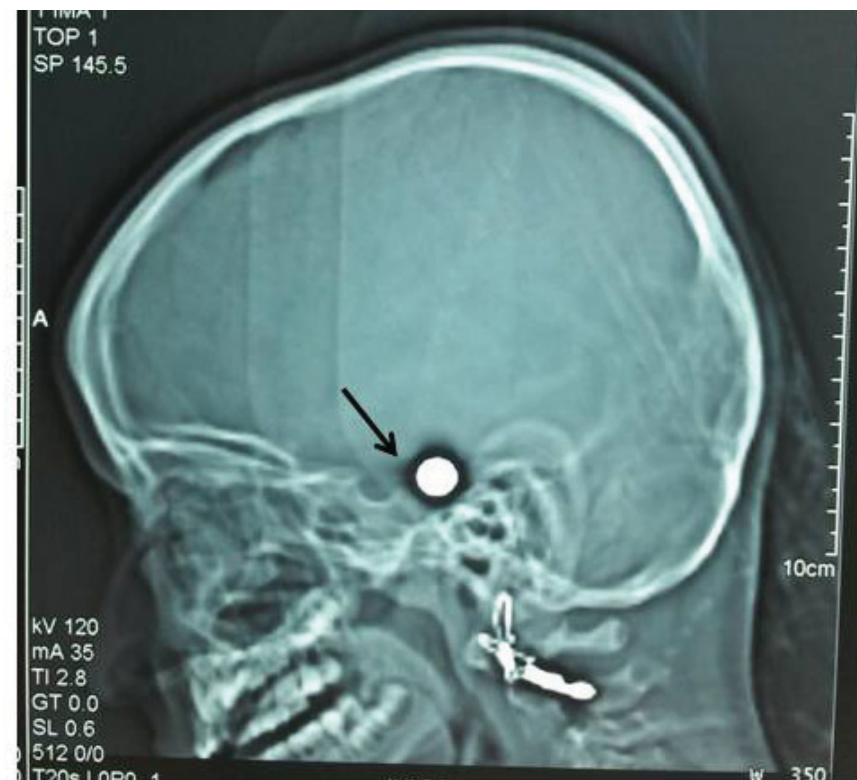

Bullet located in posterior sellar region.

\section{EP0-216 Pituitary}

\section{Childhood and adolescents brain tumors: a \\ cohort of $\mathbf{5 3}$ cases}

Nora Soumeya Fedala'; Farida Chentli'; Ale el Mahdi Haddam ${ }^{2}$ ${ }^{1}$ Beo Hospital, Endocrinology, Algiers, Algeria; ${ }^{2}$ Eph Bologhine, Endocrinology, Algiers, Algeria

Background: Brain tumors are the most common solid tumors of childhood and adolescents. The incidence is 2,5 per 100000 per year and they represent the second cause of neoplasm after leukemia. In african contries, their incidence is unknown and only a few studies have been reported.

Objective: The aim is to report the clinical and biological caracteristics of brain tomors in chilhood and adolescents seen and managed in our endocrine department

Method: Medical files of 53 patients ages between 33 months and 19 years who presented to our endocrine department for further endocrine investigation the ten years laters were reviewed to extract information about neurophtalmic ,endocrine impact, tumor size, aetiologic and therapeutic results Results: Brain tumors recount for $1,59 \%$ of pathologies and $10,15 \%$ of all intracranial tumors . A femal predominance $(54 \%)$ was observed .The main age was 15,4 years (33 months- 19 years) with a predominance of $15-19$ years age group $(47 \%)$.Meantime of diagnosis is 20 months. The most frequent motifs of consultation are endocrine signs: pituitary deficiency $34 \%$, insipidus diabetes $41,5 \%$ Clinical signs are dominated by endocrine (hypo hypersecretion $81 \%$ ) and neurophtalmic disorders $73 \% .98 \%$ of brain tumors were located in supratentorial region and represented by craniopharyngioma $(50,9 \%)$ and pituitary adenomia $(35,6 \%) .62,2 \%$ of them are huge (superior to $2 \mathrm{~cm}$ ). Surgical resection indicated in $73.6 \%$ of cases as first line was disappointing therefore a complementary treatment is given (medical and or radiotherapy)

Conclusion: Brain tumors in childhood and adolescents in endocrine practice are rare but life threating. The diagnosis is often made with delay and patients seen with complications. This can be explain by difficulties to recognize clinical symptoms in infants.. A medical education of practionners regarding presumptive signs of the disease is necessary for a precocious and an effective management of brain tumors in childhood and adolescents in our contry.

\section{EP0-217 Puberty and Neuroendocrinology}

\section{Head nodding syndrome in Northern Uganda.}

\section{Could it be a neuroendocrine problem?}

Beatrice Mpora Odongkara'; George Mulong'; David Lagoro Kitara ${ }^{3}$; Denis Arony Anywar'; Sylvia Awor ${ }^{\circ}$; James Henry Obol ${ }^{\circ}$

${ }^{1}$ Gulu University, Paediatrics and Child Health, Gulu, Uganda;

${ }^{2}$ Gulu University Faculty of Science, Chemistry, Gulu, Uganda;

${ }^{3}$ Gulu University, Faculty of Medicine, Surgery, Gulu, Uganda; ${ }^{4}$ Gulu University Faculy of Medicine, Biomedical Laboratory Technology/ Laboratory Science, Gulu, Uganda; ${ }^{5}$ Gulu University, Faculty of

Medicine, Obstetrics and Gynecology, Gulu, Uganda; ${ }^{6}$ Gulu University

Facculty of Medicine, Public Health, Gulu, Uganda

Background: The Head nodding syndrome is a mysterious condition affecting children aged $5-15$ years across northern Uganda. The disease, first reported in September 2009 among formerly displaced persons, is fast spreading across the region with close to 8000 cases and hundreds of deaths reported. The disease found in areas with O.volvulus infestation to date, has no known incidence, etiology and treatment despite investigative efforts by the CDC and Uganda's Ministry of Health. Affected children, mostly aged between 5-15 years, present with head nodding at the sight of food which stops when food is withdrawn, progressing to seizures, growth failure, mental retardation and eventually death. These symptoms worsen with coldness and improve on warming the victims. Of all investigations done, no mention has been made of possible neuro-endocrine causes of the disease. Symptomatic treatment with anticonvulsants has yielded no results. In Russia, similar conditions were treated in the 1970s with both anticonvulsants and ACTH with some improvement. There is need for in-depth investigation into the neuroendocrine functions among the affected children.

Objective and hypotheses: The main objectives of the investigation are to ascertain the neuro-endocrine causes/complications of nodding syndrome and put in place cost effective interventions to alleviate sufferings in the affected children and families.

Methods: This will be a case control study with prospective cohort study using both qualitative and quantitative methods of data collection.

Results: The results is expected to inform the ministries of health and health care workers on putting in place cost effective interventions to combat the disease and alleviate symptoms while promoting optimal growth among the affected children.

Conclusions: There is urgent need to combine efforts by all stakeholders including research scientists to find the cause and lasting solutions to the this strange and rare epidemic of head nodding syndrome that has afflicted children and adolescence in this formerly war affected region.

\section{EPO-218 Puberty and Neuroendocrinology \\ Age of puberty and its relationship with body mass index in girls living in Tabriz}

Robabeh Ghergherehchir'; Nazanin Hazhir ${ }^{2}$

${ }^{1}$ Tabriz University of Medical Sciences, Pediatric Endocrinology,

Tabriz, Islamic Republic of Iran; ${ }^{2}$ Tabriz University of Medical Sciences, Pediatrics, Tabriz, Islamic Republic of Iran

Background: Evaluation of puberty levels in a population requires exact reference values for comparison.

Objective and hypotheses: This study aimed to determine the age of puberty onset in girls living in Tabriz, Iran and its relation to body mass index (BMI) Methods: This cross-sectional study was conducted on 1000 female students, aged 7-17 years, in Tabriz between 2008 and 2009. Participants were selected by multistage random cluster sampling from school students. According to BMI, the participants were divided into 4 groups: obese, overweight, normal weight and underweight. Secondary sexual characteristics were evaluated by inspection and palpation according to Tanner staging. Menarche age, if any, was also recorded.

Results: The mean age of puberty onset (breast stage II) was $9.58 \pm 1.15$ years and the mean age of tanner stage II of pubic hair (P2) was $10.30 \pm 1.1$ years. The mean age of menarche was $12.58 \pm 1.30$ years. There was significant difference between the age puberty onset and BMI $(\mathrm{P}=0.002)$. The mean menarche age in the underweight and normal weight groups was significantly greater than overweight and obese groups ( 14.85 vs 13.81 years, $\mathrm{P}<0.001)$.

Conclusions: Age of puberty onset is decreasing in our community. This study confirms that obese and overweight girls reach the puberty at a lower age than normal weight girls and their menarche age is less as well. 


\section{EPO-219 Puberty and Neuroendocrinology}

Different clinical courses of central precocious girls according to the age at presentation and treatment

Jaehong Yu'; Heon-Seok Han²

1Joy Children Hospital, Pediatrics, Daejeon, Republic of Korea; ${ }^{2}$ Chungbuk Nation University Hospital, Peidatics, Cheongju, Republic of Korea

Background: Progressive puberty with a growth spurt ensues when activation of the pituitary-ovarian axis is sustained. However, puberty is not necessarily intense enough or sustained enough to cause the inexorable progression of normal puberty or to cause deterioration of height potential.

Objective and hypotheses: The progressivity of central precocious puberty seemed to depends on the age at presentation. We investigated the clinical courses between early onset and late onset groups.

Methods: One hundred thirty five girls with central precocious puberty (CPP) diagnosed between Jan. 2003 and Dec. 2009 were included. Among 135 patients, 123 were idiopathic CPP and twelve had neurogenic problems. CPP criteria were development of secondary sexual characteristics before $8 \mathrm{yr}$ of chronologic age (CA) with advanced bone age (BA), and stimulated $\mathrm{LH}>5$ IU/L. They were treated with GnRH analogues and followed for more than 1 yr. Subjects were divided into two groups if they were treated before (Group I) or after 7 yr of age (Group II).

Clinical courses and laboratory findings were evaluated every 6 months. We compared anthropometric parameters, calculated predicted adult height $(\mathrm{PAH})$, predicted treatment periods, and laboratory findings.

Results: 1) Among the baseline parameters, BA and CA were greater in group II, but $\mathrm{BA} / \mathrm{CA}$ ratio were significantly greater in group $\mathrm{I}$.

2) The time needed for disappearing the CA and BA difference was 4.64 $\mathrm{yr}(3.74 \sim 5.54 \mathrm{yr})$ for the total patients, but the time is significantly different between the two groups, $7.98 \mathrm{yr}(3.88 \sim 12.07 \mathrm{yr})$ for group I and $4.24 \mathrm{yr}$ (3.74 4.73 yr) for group II.

3) The time needed for disappearing the PAH and TH difference was $2.49 \mathrm{yr}$ (1.96 3.01 yr), but the time is significantly different between the groups, 4.37 yr (0 12.58 yr) for group I and $2.32 \mathrm{yr}(1.48 \sim 3.16 \mathrm{yr})$ for group II.

Conclusions: Among the girls with CPP, younger age group had more advanced bone age compared to chronologic age, and needed significantly longer treatment periods for the disappearance of BA-CA gap and PAH-TH gap.

\section{EPO-220 Puberty and Neuroendocrinology \\ A pilot study on children with bone-age advancement without early sexual maturation; auxological and laboratory (GnRH stimulation) characteristics \\ Cheol Woo Ko; Byung Ho Choi; Hyo Min Park \\ Kyungpook National University Hospital, Pediatrics, Daegu, Republic of Korea}

Background: Social concern over precocious puberty (PP) is rising nowadays, and there is actually increased visit to hospital due to PP. Among them children with no pubertal sign showing advanced bone-age(BA) have been found in many cases.

Objective and hypotheses: This study was done to see clinical and laboratory characteristics in these children with so called 'asymptomatic PP'

Methods: Among children whom visited for evaluation of early sexual maturation between July 2009 and June 2011, children with advanced BA more than $1 \mathrm{yr}$ without any pubertal sign were enrolled. Their clinical, laboratory data including GnRH stimulation test and auxological data were analyzed retrospectively.

Results: Thirty-eight children with asympomatic PP were enrolled. Male:female ratio was $1: 1$. Chronological ages (CA) were $9.3+/-1.1$ yrs in boys, $8.1+/-1.4$ yrs, in girls. BA were $11.3+/-0.9$ yrs in boys, $9.8+/-1.4$ yrs in girls. Positive result (peak $\mathrm{LH}>5 \mathrm{IU} / \mathrm{L}$ ) of $\mathrm{GnRH}$ stimulation test was found in 18 out of 38 children (47.4\%) - 18 boys (69\%) and 8 girls (31\%).

Patients were divided into 2 groups (positive and negative group) based on $\mathrm{GnRH}$ stimulation test. Basal LH value of positive group was significantly higher than negative group in boys $(0.4+/-0.5$ vs $0.1+/-0.1 \mathrm{IU} / \mathrm{L}$, respectively, $\mathrm{p}<0.05)$ and girls $(1.0+/-1.7$ vs $0.1+/-0.0 \mathrm{IU} / \mathrm{L}$, respectively, $\mathrm{p}<0.05)$.

However, there were no significant differences between groups in height, chronological age, progression of BA, BMI.
Conclusions: Children with BA advancement without secondary sexual characteristics was found. Their clinical course is unknown yet. Further study is necessary to confirm chracteristics of BA advancement without early sexual maturation, so-called 'asymptomatic PP'.

\section{EPO-221 Puberty and Neuroendocrinology \\ Clinical characteristics of the girls presented with early maturation}

Phil Soo Oh; Hui Kwon Kim

Hallym University Medical Center, Pediatrics, Chuncheon, Republic of Korea

Background: In Korea, there are many concerns about precocious puberty and short stature.

Objective and hypotheses: The purpose of this study is to analyze the clini$\mathrm{cal}$ and environmental characteristics of early mature girls by physical examinations and questionnaire.

Methods: This study was done prospectively for 158 early mature girls in the Department of Pediatrics, Hallym University Medical Center from July 2008 to June 2010.

Results: 1 . For the 158 girls, the mean ages are $8.27 \pm 1.3$ years and mean bone age advancement is $1.86 \pm 0.27$ years. 2 . In considerable cases, their weight-toheight percentiles is in $25 \sim 50(27 \%)$ or $50 \sim 75(26.3 \%)$. 3. They have normal/ good balanced or meat-prone appetite in most cases (91\%). 4. Family history of early maturation was about $40 \%$ positive. And in family relationship, the first girls have a tendency to be mature early twice more than in second. 5 . Herb medicine taking history with ginseng was up to $67.5 \%$.

Conclusions: 1 . Unexpectedly, their weight-to-height percentiles is considerably in $25 \sim 50(27 \%)$ or $50 \sim 75(26.3 \%)$. This seems to be a possibility of any other causes for precocious puberty in addition to body fatness. 2 . In family relationship, the first girls interestingly have a tendency to be mature early twice more than in second 3 . Herb medicine taking history with ginseng was up to $67.5 \%$. With ginseng's phytoestrogenic effect in some reports, herb medicine seems to be an important factor for early maturation in Korea.

\section{EPO-222 Puberty and Neuroendocrinology \\ Gonadotropin-releasing hormone (GnRH) stimulation test before and after GnRH analogue treatment in central precocious puberty; can GnRH test simplify adequately? Min Sun Kim; Pyoung Han Hwang; Dae-Yeol Lee \\ Chonbuk National University Medical School, Pediatrics, Jeonju-si,} Republic of Korea

Background: Gonadotropin-releasing hormone (GnRH) stimulation test is the gold standard to document premature activation of the hypothlamic-pituitary-gonadal axis in early puberty. However, this test is time-consuming, costly and uncomfortable for the patients.

Objective and hypotheses: The aim of this study was to investigate to simplify the GnRH stimulation test in the assessment of pubertal activation and suppression.

Methods: We identified 72 girls diagnosed with central precocious puberty, and they were treated with GnRH analogue $(\mathrm{GnRHa})$ to suppress pubertal progression from 1 January 2011 to 31 January 2012. GnRH stimulation tests were done before and 4 weeks after the third dose of the GnRH analogue. Results:

1) Before GnRHa treatment, the mean luteinizing hormone (LH) level was higher at the 30 th minutes $(18.17 \mathrm{IU} / \mathrm{L})$ of the test in comparison to the basal level $(0.35 \mathrm{IU} / \mathrm{L})$, the 15 th, 60 th, 90 th and 120 th minutes $(\mathrm{p}<0.001)$. However, there was no significant difference in the $\mathrm{LH}$ level between the 30th and 45 th minutes of the test. Among 72 patients, 49 girls $(68.1 \%)$ were showed the peak LH level at 30th minutes and the others were showed the peak LH level at 45 th minutes $(33.3 \%)$, and 60th minutes $(6.9 \%)$.

2) After GnRHa treatment, 62 patients $(86.1 \%$ ) were suppressed (peak LH level $<2$ IU/L), and $10(13.9 \%)$ were inadequately suppressed. The LH level was higher at the 30 th minutes $(0.91 \mathrm{IU} / \mathrm{L})$ of the test in comparison to the basal level $(0.26 \mathrm{IU} / \mathrm{L})$, the 45 th minutes $(0.78 \mathrm{IU} / \mathrm{L})$ and 60 th minutes $(0.71$ $\mathrm{IU} / \mathrm{L})(\mathrm{p}<0.001)$

But the LH level was no significant difference between 15th and 30th minutes. The AUC in ROC analysis was greatest at the 30 th minutes. The basal 
LH level of the test was showed low sensitivity (25.0\%).

Conclusions: It is adequate to check the LH level at 30th and/or 45th minutes of the GnRH test for diagnosis of CPP and 30th minutes for assessment of pubertal suppression. Therefore we suggest that the simplified GnRH test is sufficient to evaluation of pubertal activation and suppression.

\section{EPO-223 Puberty and Neuroendocrinology \\ Pubertal characteristic in girls of Qazvin province Iran}

Fatemeh Saffari; Maryam Rostamian; Neda Esmaeilzadehha;

Keyvan Shariatinejad; Toktam Karimzadeh

Qazvin University of Medical Sciences, Pediatrics, Qazvin, Islamic

Republic of Iran

Background: Puberty is a critical time between childhood and adulthood. Puberty onset is determined by the appearance of breast buds in girls. Many studies have been reported that the mean age of breast development is decreasing.

Objective: The aim of this study was to provide updated data on the pubertal development of girls and to evaluate precocious puberty in our population.

Methods: This cross sectional study was conducted in 6 to 16 years old school girls during 2009 to 2010 in Qazvin. 2240 healthy girls from all geographical regions with every socioeconomic status were selected by a stratified multistage cluster design to obtain representative sample of population. A questionnaire including demographic data, anthropometric measurements, secondary sexual characteristics, menarche status and its onset was filled out for every participant. Secondary sexual characteristics including breast development (B1-5) and pubic hair (PH 1-5) were evaluated according to Marshal and Tanner recommendation.

Results: The mean \pm SD of height, weight, and BMI of participants was $139.7 \pm 14.5,36.1 \pm 12.9$ and $17.9 \pm 3.7$ respectively.

The mean age (10th - 90th percentile) of B2 and PH2 were 9.71(7.67 - 11.4) and 9.82 years $(7.84-11.42)$ respectively. Mean age of menstruation was 12.52 years.

The mean BMI was significantly higher in pubertal females comparing to prepubertal girls $(p<0.001)$. Average duration of puberty (the time from initiation of puberty to menarche) was 2.81 .

Conclusion: The mean age of pubertal onset in girls living in Qazvin is 9.71 years. Menarche occurs at mean age of 12.52 and onset of puberty earlier than 6.24 years will be precocious. We found that girls in Qazvin had a slightly earlier age of initiation of puberty and of menarche in comparison with other studies in Iran.

\section{EPO-224 Puberty and Neuroendocrinology \\ Precocious puberty in the context of hepatoblastoma and established renal failure Lynn Diskin ${ }^{1}$; Christine Burren²; Jan Dudley ${ }^{1}$ \\ ${ }^{1}$ Bristol Royal Hospital for Children, Department of Paediatric Nephrology, Bristol, United Kingdom; 'Bristol Royal Hospital for Children, Department of Paediatric Endocrinology, Bristol, United Kingdom}

Background: Precocious puberty secondary to hepatoblastoma is a rare but well-recognised association, with approximately 20 reported cases.

Hypothesis: This case features unusual results suggesting novel mechanisms triggering the precocious puberty.

Methods: A 2 year 9 month old boy, with a complex medical history including established renal failure, presented with progressive virilisation over 3 months. Examination revealed genitalia stage 3, pubic hair stage 2, $4 \mathrm{ml}$ testes and height on 25th centile. Virilisation was excessive for testicular volumes, suggesting potential adrenal pathology.

Investigations: Revealed normal baseline and stimulated adrenal steroids and normal urine steroid profile, thus excluding non-classical congenital adrenal hyperplasia or abnormal adrenal steroid production. Testosterone was elevated $(6.4 \mathrm{nmol} / 1$; normal $0.2-1.7 \mathrm{nmol} / 1)$. GnRH test results are below.

\begin{tabular}{|lccccc|} 
& Baseline & $\begin{array}{c}20 \\
\text { minutes }\end{array}$ & $\begin{array}{c}60 \\
\text { minutes }\end{array}$ & $\begin{array}{c}\text { Normal } \\
\text { Baseline }\end{array}$ & $\begin{array}{c}\text { Normal } \\
\text { Stimulated }\end{array}$ \\
\hline Lutenising Hormone (IU/L) & 3.7 & 16.5 & 23.2 & $0.2-0.5$ & $<6$ \\
\hline Follicle Stimulating Hormone (IU/L) & 9.3 & 14.5 & 21.2 & $0.2-4.7$ & $1-11.6$ \\
\hline
\end{tabular}

Results: Results indicated central precocious puberty and treatment was commenced with GnRH analogue (Gonapeptyl), and anti-androgen (Cyproterone Acetate). MRI imaging revealed normal pituitary appearances and, unexpectedly, 3 hepatic masses. Biopsy confirmed hepatoblastoma. Serum AFP was moderately elevated, but $\beta$-hCG normal. Repeat GnRH test at 12 weeks showed no rise in stimulated LH levels. Despite an excellent tumour response to chemotherapy, one lesion was inoperable. Liver and renal transplant was therefore undertaken with good outcome.

Conclusions: Almost all previously reported cases are associated with $\beta$-hCG secretion by tumour cells. $\beta$-hCG mimics LH in stimulating testicular testosterone secretion, thus resulting in gonadotrophin-independent precocious puberty. Unusually in this case $\beta$-hCG levels are normal and the hypothalamic-pituitary-gonadal axis is also stimulated. Proposed mechanisms are that tumour cells are secreting either testosterone or a $\beta$-hCG-related product that is unrecognised by current assays. It is thought that the HPG axis is stimulated by high circulating testosterone levels.

\section{EPO-225 Puberty and Neuroendocrinology \\ Clinical characteristics of girls with early puberty and effect of GnRHa treatment on height depending on the bone age: a-two- year follow-up}

Senay Savas Erdeve; Merih Berberoglu; Bulent Hacihamdioglu; Zeynep Siklar; Emine Camtosun; Gonul Ocal

Ankara Univesity School of Medicine, Department of Pediatrics, Division of Pediatric Endocrinology, Ankara, Turkey

Background: There are few studies on the effect of GnRHa treatment on height in early puberty.

Objective and hypotheses: To define the characteristics at application in early puberty, the effect of GnRHa treatment on short-term predicted adult height (PAH) by comparing to control group, and whether timing of treatment has advantage or not in patients with early bone age (BA) when compared to patients with advanced BA.

Methods: Girls with early puberty (7-8.5 years) were divided into 2 groups; patients with $\mathrm{BA} \leq 10.5$ years composed Group $1(\mathrm{n}=26)$, whereas others with $\mathrm{BA}>10.5$ years composed Group $2(\mathrm{n}=28)$. Patient who did not accept treatment composed the control group $(\mathrm{n}=14)$.

Results: Patients had mean age of 8.53 years and pubertal stage 3 at application. Obesity and overweight rates were $10.3 \%$ and $50.3 \%$, respectively. Treatment and control groups did not show significant differences in target height (TH), PAH and SDS at diagnosis. Height SDS was not statistically different in treatment and control groups at diagnosis. In treatment groups, height SDS was greater in Group 2 when compared to Group 1. This difference disappeared at the end of 1st and 2nd years of the treatment. Body mass index SDS were similar in three groups both at diagnosis and at the end of the first year. $\Delta \mathrm{BA} / \Delta$ chronological age was statistically lower in treatment groups in comparison to control group. There were not statistically significant differences in first year PAH and SDS of treatment and control groups. Second year PAH and SDS of treatment groups were similar. In each group, PAH and SDS did not show difference in TH both at diagnosis and on follow-up.

Conclusions: Similarity of PAH to TH in girls with early puberty both in control and treatment groups on follow-up suggest that patients in that age group has potential to reach genetic $\mathrm{TH}$ without treatment.

\section{EPO-226 Puberty and Neuroendocrinology \\ Charge syndrome in a Nigerian infant: a rare genetic syndrome, case report \\ Olumide Jarrett; Kehinde Fashina}

University College Hospital, Paediatrics, Ibadan, Nigeria

Background: Charge syndrome is a rare genetic disorder that arises during early fetal development and affects multiple organ systems. Diagnosis is largely clinical. Mutation at the CHD7 gene located on Chromosome 8 has been identified in a great number of patients reviewed in different parts of the world. Survival depends on the intensity of the medical management as well as an early aggressive approach to the feeding adaptation in these children.

Case report: We report a case of a 42 day old baby with clinical features in keeping with Charge syndrome. He was a product of a full term uneventful pregnancy period delivered to non consanguineous apparently healthy 
parents. Two older siblings were normal. He developed respiratory distress shortly after birth. Multiple abnormalities were identified at birth which included genital (cryptochidism), ear (microtia), eye(micro-ophthalmia) and cardiovascular as well as skeletal (polydactyly) abnormalities. Genetic testing was not carried out due to cost. Child was managed by a multidisciplinary team. Main problems were those of sepsis and feeding adaptation. He later succumbed to death after a month on admission.

Conclusions: This is the first case of Charge syndrome reported in Nigeria. Charge syndrome though rare could be easily missed if not aware of the existence of such a condition. This report is to increase awareness of this rare condition and to promote better intervention of similar presentation in future.

\section{EPO-227 Puberty and Neuroendocrinology \\ A case of familial male-limited precocious puberty - a lesson on red herring, and variable expressivity}

Yvonne, Yijuan Lim; Cindy, Wei-li Ho; Yung Seng Lee

National University Hospital, University Children's Medical Institute,

Singapore, Singapore

Background: Familial male-limited precocious puberty (FMPP) is a rare cause of gonadotropin-independent isosexual precocious puberty in male. It is due to activating mutation of the $\mathrm{LH}$ receptor, leading to secretion of testosterone by the Leydig cells. Affected males usually present at about 1-4 years old with rapid growth and progressive virilisation. If untreated, FMPP can result in behavioural problems and compromised final height due to premature epiphyseal fusion.

Results: Patient A with underlying Neurofibromatosis Type 1 (NF1) presented with precocious puberty (PP) at 5 years of age, with tall stature, advanced bone age of 9 years, and testicular volume of 6-7 mls, but endocrine assessment indicated gonadotropin independent precocious puberty. We found a heterozygous missense mutation in the LHCGR gene A572V which was previously reported elsewhere, confirming the diagnosis of FMPP. His father was also heterozygous for this mutation but he was asymptomatic, with normal Asian adult height of $170 \mathrm{~cm}$ and no apparent PP during his childhood.

Conclusions: FMPP is uncommon and the diagnosis may be difficult to establish initially, especially so in this case as central or gonadotropin dependent precocious puberty was more commonly associated with NF1. His father was apparently an asymptomatic, and there was no family history of PP. This is likely due to variable expressivity, and also modified by the genetic height potential of the individual.

\section{EPO-228 Puberty and Neuroendocrinology \\ Precocious puberty in children}

Irum Tufail; Taj Muhammad Laghari; Yasir Naqi khan;

Saira Waqar lone; Mohsina Ibrahim; Jamal Raza

National Institute of Child Health, Medical Unit II, Karachi, Pakistan

Background: Normal pubertal development is one of prime concern of parents of a growing child. The precocious puberty has significant psychosocial impact on child and parents. It influences psychosocial development and growth of child.

Objective and hypotheses: Evaluate and determine the etiology of precocious puberty at tertiary care hospital and to compare the clinical and laboratory parameters of central and peripheral precocious puberty.

Methods: Children who fulfilled the criteria of precocious puberty were included. Precocious puberty defined as the development of secondary sexual characteristics before the age of 8 years in girls and 9 years in boys. All patients evaluated clinically and on laboratory investigations. Data was entered and analyzed using SPSS version 17.0. Independent sample t-test/ MannWhitney U-test were applied.

Results: Total numbers of patients registered during this period were 84 . The conditions causing precocious puberty were central precocious puberty $(36.5 \%)$, peripheral precocious puberty $(38.8 \%)$, premature pubarche $(10.6 \%)$ and premature thelarche (14.1\%). In central precocious puberty 26 were female and 5 were male. The causes identified in them were idiopathic (67.74\%), hypothalamic hamartoma $(12.90 \%)$, craniopharagioma $(9.67 \%$, arachnoid cyst $(3.22 \%)$, hypothalamic astrocytoma $(3.2 \%)$, hydrocephalus $(3.2 \%)$. In peripheral precocious puberty 21 were male and 12 were female. Congenital adrenal hyperplasia $(81.8 \%)$, adenocarcinoma $(9.1 \%)$, ovarian teratoma (6.1\%) and Mc Cune Albright syndrome (3\%) were diagnosed in them. There was difference in the age of onset of puberty of central precocious puberty 3(2-6) versus peripheral precocious puberty 5.25(3.62-7.0). Central precocious puberty children showed higher height SDS, weight SDS, BMI, FSH, LH and bone age than peripheral precocious puberty.

Conclusions: Peripheral precocious puberty is more common than central precocious puberty. Height SDS, weight SDS, BMI,FSH,LH were higher in central precocious puberty versus peripheral precocious puberty.

\section{EPO-229 Puberty and Neuroendocrinology \\ The median age of the onset of puberty in healthy Uzbek boys of Tashkent city}

Gulnara Rakhimova; Kamil Gilyazetdinov; Anvar Akbarov

Republican Specialized Scientific-practical Medical Centre of

Endocrinology, Children department, Tashkent, Uzbekistan

Background: The age of the onset of puberty is much lower today than in the past century, due to the stability of the socio-economic conditions, improved quality of life and overall health of the population.

Materials and methods: We examined healthy 818 boys aged from 7 to 18 years, the study was conducted between 2009 and 2011. Boys were examined in 3 urban districts of Tashkent city i.e. (Mirzo-Ulugbek, Shakhantaur, YunusAbad). Testicular volume was evaluated with a Prader orchidometer. The age of the onset of puberty defined as the age at attainment of testicular volume of $4 \mathrm{~mL}$ or greater.

Results: We revealed that the earliest age of the onset of puberty was at 9.0 yr.(3.3\%).All healthy boys had testicular volume greater than $4 \mathrm{ml}$ by 14 years. The data suggest for healthy Uzbek boys is defined upper reference value of the age of the onset of puberty. Thereby the diagnosis of delayed puberty may be established at 14 years, which generally coincides with the international guidelines for the diagnosis of delayed sexual development. The median age of the onset of puberty for Uzbek boys of Tashkent city was 11.9 (95\% CI 11.1-12.7), this data agree with Great Britain (11.9) and Byelorussia (11.9), but different from USA(11.5), Netherlands(11.5).

Conclusion: 1. The earliest age of the onset of puberty in healthy Uzbek boys of Tashkent city was $9.0 \mathrm{yr}$. and $14 \mathrm{yr}$. ultimate one.

2. The median age of the onset of puberty for Uzbek boys of Tashkent city was 11.9 yr.

3. We have defined criteria for the diagnosis of delayed puberty at 14 years.

\section{EPO-230 Puberty and Neuroendocrinology \\ Aetiological evaluation of patients presenting with micropenis to an academic health care centre \\ Fatih Gurbuz; Tugba Bilmez Aslan; Fatih Temiz; Eda Mengen; \\ Bilgin Yuksel; Ali Kemal Topaloglu \\ Cukurova University, Pediatric Endocrinology, Adana, Turkey}

Background/aims: The objective of this study was etiological evaluation of the patients presented to a tertiary health care center with micropenis.

Methods: In all patients who were referred with a diagnosis of micropenis from October 2009 through October 2010, a complete evaluation including measurement of stretched penile length was carried out.

Results: In 20 (31\%) among a total of 65 patients, measured stretched penile length was not consistent with the description of micropenis. True presence of micropenis was confirmed in the remaining 45 of total 65 cases $(69 \%)$. Etiological cause was determined in 29 patients (44\%) with the most common cause being hypogonadotropic hypogonadism. No etiological cause could be found in 16 patients $(25 \%)$.

Conclusions: In approximately one third of patients the presence of micropenis was not confirmed. The most common causes of misdiagnosis are buried penis due to obesity, erroneous measurement of stretched penile length, and/ or lack of knowledge on population standards for penile length. Etiological basis of micropenis is rather heterogeneous. Despite extensive investigations, no causal link was found in around one fourth of cases. Accurate measurement and utilization of internationally accepted standards is utmost importance for diagnosis and management of micropenis. 


\section{EPO-231 Puberty and Neuroendocrinology}

Two sporadic patients of Perrault syndrome with ovarian dysgenesis and sensorineural deafness

Young-Lim Shin

Soonchunhyang University Bucheon Hospital, Pediatrics, Bucheon, Republic of Korea

Background: Perrault syndrome is an autosomal recessive disorder characterized by sensorineural deafness and ovarian dysgenesis. Some patients also have neurologic abnormalities, including cerebellar ataxia, nystagmus, polyneuropathy and mild mental retardation. The syndrome is known to be caused by mutations in HSD17B4 or HARS2 until now but few patients were reported.

Objective and hypotheses: We report on two sporadic Korean patients of Perrault syndrome with ovarian dysgenesis and sensorineural deafness.

Method and results: Patient 1: A 14 year-6 month female patient presented with delayed puberty. She had bilateral sensorineural hearing impairment. Her neurological findings were normal and She had no mental retardation. She had normal karyotype $(46, \mathrm{XX})$ and hypergonadotropic hypogonadism. Pelvic ultrasound showed a small uterus and ovaries. Brain MRI was normal. Patient 2: A 16 year-11 month female presented with delayed puberty and neurologic manifestations. She had ataxia, dyspraxia and weakness. She had no mental retardation and brain MRI was normal. She had multiple Cafe au lait spots and long slender fingers but no Marfanoid features. Laboratory tests revealed hypergonadotropic hypogonadism and normal female karyotype (46, XX). Pelvic ultrasound examination showed a hypoplastic uterus and small ovaries.

Conclusions: Because Perrault syndrome has clinical variability and genetically heterogeneous, we have to inspect additional findings and neurological abnormalities. And further studies will be required to ascertain the common causative mutation of this syndrome.

\section{EPO-232 Puberty and Neuroendocrinology}

\section{Hormonal and anthropometric factors associated with pubertal gynaecomastia Ibrahim Al Alwan'; Motasim Badri'; Waleed Tamimi2;} Mohammed Al Dubayee ; Hani Tamim ${ }^{1}$; Hanan Al Azkawi ${ }^{1}$ King Saud bin Abdulziz University for Health Sciences, College of Medicine, Riyadh, Saudi Arabia; ${ }^{2}$ King Saud bin Abdulziz University for Health Sciences, College of Applied Medical Sciences, Riyadh, Saudi Arabia; ${ }^{3}$ King Abdulaziz Medical City, Pediatrics, Riyadh, Saudi Arabia

Background: Gynecomastia is the glandular proliferation of male breast tissue. It is a complex process and various hypotheses have been suggested to explain it.

Objective: To evaluate the association of hormonal levels and anthropometric values with pubertal gynecomastia.

Method: A cross sectional study among healthy male school children in Riyadh, Saudi Arabia. A total of 542 aged 6 to 16 years old were selected from diverse socio-economic backgrounds. Tanner stage, height, weight, Blood hormonal levels (Leutilizing Hormone (LH), Folicular Stimulating Hormone (FSH), total Testosterone and Estradiol) well as anthropometric data (BMI, Triglycerides, High Density Lipoprotein (HDL), Low Density Lipoprotein (LDL) were collected and compared in children with gynecomastia (diffused or firm) and those without.

Results: Gynecomastia was found in 185/542 (34\%) of the children studied. Median (IQR) age in the whole group was 11(8-13) years. The two groups compared had non-significant cholesterol $(p=0.331)$, LH $(p=0.215),(F S H$ $(\mathrm{p}=.571)$ levels. In multivariate analysis, factors significantly associated with gynecomastia were gonad (Stage II Odds Ratio [OR] 2.04;95\% CI 1.50$3.61 ; \mathrm{p}=0.015$,Stage III OR=5.39; 95\%CI 2.26-12.8; $\mathrm{p}<0.0001$, Stage IV $\mathrm{OR}=2.66 ; 95 \%$ CI 1.06-6.66; $\mathrm{p}=0.037$, Stage V OR $=1.10 ; 95 \%$ CI $0.41-2.96$; $\mathrm{p}=0.84$, compared with stage I), Body Mass Index $[\mathrm{BMI}](\mathrm{OR}=1.05 ; 95 \% \mathrm{CI}$ $1.01-1.08 ; \mathrm{p}=0.16)$ and $\mathrm{HDL}(\mathrm{OR}=0.42 ; 95 \%$ CI $0.19-0.96 ; \mathrm{p}=0.039)$.

Conclusion: Pubertal gynecomastia tends to increase in mid-puberty. A number of factors are associated with the development of Gynecomastia in pubertal boys. BMI, HDL and Gonad stage are the major contributing factors found in our study.

Key words: Puberty, gynecomastia, hormonal levels children

\section{EPO-233 Puberty and Neuroendocrinology \\ Impact of gonadotropin-releasing hormone analogue treatment in children with idiopathic central precocious puberty: a single-centre study \\ Kyung-Sue Shin \\ Jeju National University School of Medicine, Pediatrics, Jeju, Republic of Korea}

Background: The long-term outcomes of Gonadotropin-releasing hormone analogue (GnRHa) treatment on body mass index (BMI), body composition, and reproductive function are still under debate.

Objective and hypotheses: The outcomes of GnRHa treatment in idiopathic central precocious puberty (ICPP) were evaluated.

Methods: Thirty-six girls with ICPP treated with GnRHa from 2002 through 2008 were enrolled. Another 12 girls, who had not treated with GnRHa for several causes, were also included for controls. Bone age (BA), predicted adult height $(\mathrm{PAH})$, body mass index (BMI) and time of menarche were obtained retrospectively.

Results: Mean chronologic age and BA of GnRHa-treated group before treatment were was $8.2 \pm 0.8$ years and $10.4 \pm 1.5$ years, respectively. PAH after treatment was increased to $156.1 \pm 6.7 \mathrm{~cm}$ (before treatment, $154.6 \pm 6.4 \mathrm{~cm}$ ). PAH of the treated group was greater than untreated group $(155.2 \pm 5.9 \mathrm{~cm})$. BMI (18.1 \pm 2.5 vs. $19.4 \pm 3.1)$ and BMI percentile $(78.4 \pm 18.7 v s .83 .9 \pm 14.8)$ were increased at the end of treatment, but BMI was normalized near the time of adult height achievement. Menarche occured after GnRHa discontinuation for $1.2 \pm 0.4$ years.

Conclusions: GnRHa treatment in ICPP is relatively safe and reversible for the pubertal development. It may improve the final height close to PAH in children with ICPP. Further studies to improve final height in children with ICPP in various conditions were demanded.

\section{EPO-234 Puberty and Neuroendocrinology \\ Precocious puberty in Costello syndrome: case report}

Giovanni Corsello'; Silvano Bertelloni'; Maria Cristina Maggio

${ }^{1}$ University of Palermo, Materno-Infantile, of Andrology and Urology,

Palermo, Italy; ${ }^{2}$ University of Pisa, Pediatric Endocrinology, Division of

Paediatrics, Department of Reproductive Medicine and Paediatrics,

Pisa, Italy

Background: Costello syndrome(CS) is a rare autosomal dominant genetically transmitted disease, with: macrocephaly, coarse face with hypertelorism, epicanthal folds, prominent eyes, short nose, low-set ears, large mouth, short stature and failure to thrive, curly hairs, macro glossy, short neck, hyperkeratosis, hyperpigmentation, papillomata around the anus, mouth and nares, thick and loose skin of the hands and feet, cardiomyopathy, mental retardation, increased neoplastic risk. The majority of patients $(80-90 \%)$ with CS have de novo heterozygous mutations in the HRAS gene (11p15.5). Sporadic cases with GH deficiency are described, even if GH treatment is non clearly associated to a significant gain in growth. However GH treatment could deteriorate hypertrophic cardiomiopathy and increase neoplastic risk. We describe the case of a girl with CS genetically diagnosed, who showed mild mental retardation, hypertrophic cardiomiopathy, aortic stenosis, short stature, hypoplastic nails, coarse facies, short neck, papillomata around the anus, mouth and nares.

Results: She underwent to endocrine follow-up, to evaluate short stature, and a precocious puberty with insurance at 7 years. She presented a bone age of 11 years, pubertal stage: PH2B3, vaginal bleeding, FSH/LH T0 and after LHRH: 7,1/21,4 e 1,7/9,3; Magnetic Resonance Imaging negative, increased uterine and ovarian volume, with multifollicular ovaries. She started LHRH analog treatment to delay bone age maturation, menses and pubertal development, and to inhibit LH and FSH. Actually she is 10,1 years, stature: $123 \mathrm{~cm},-2,7$ DS; weight: 32,5 kg; -0,8 DS; bone age: 12 years, pubertal stage: PH1B2. Conclusions: We signal this case for the original relieve of precocious puberty in CS, otherwise frequently described in association with delayed puberty, on average at 15 years. LHRH analog treatment ameliorated growth prognosis in a patient not eligible for $\mathrm{GH}$ treatment. 


\section{EPO-235 Puberty and Neuroendocrinology \\ Diverse phenotypes of McCune-Albright syndrome}

Daniela Amaral'; Silvia Sequeira22; Rosa Pina'; Lurdes Lopes

${ }^{1}$ Hospital de Dona Estefânia, Pediatric Endocrinology Unit, Lisbon,

Portugal; ${ }^{2}$ Hospital de Dona Estefânia, Metabolics Diseases Unit,

Lisbon, Portugal

Background: McCune-Albright Syndrome (MAS) is a very rare disorder with an extended clinical spectrum. We report three cases suspected of MAS on clinical grounds.

Objective:

Case 1: 2.3 years old girl, started thelarche and vaginal bleeding. Ultrasound revealed a hemorrhagic ovary cyst that regressed spontaneously, as well as the clinical signs. Laboratory evaluation showed slightly increased estradiol$24 \mathrm{pg} / \mathrm{ml}(\mathrm{RV}:<20 \mathrm{pg} / \mathrm{ml})$ and pre pubertal LH and FSH levels on LHRH stimulation test. Thelarche and vaginal bleeding waxed and waned. Cranial MRI revealed polyostotic lesions;skeletal study was normal. Recently, at 8 years of age, two major bleeding periods occurred with high levels of estradiol $(953 \mathrm{pg} / \mathrm{ml})$. She has no other manifestations of MAS.

Case 2: 9 years old African girl sent for evaluation of recurring fractures, severe bone deformities (bilateral coxa vara) and large "café au lait" maculas. Menarche had occurred a fortnight before. Skeletal survey revealed several healed fractures, polyostotic lesions, osteosclerosis and ground glass images of the skull. Laboratory evaluation revealed: PTH:26.4pg/ml (RV:12-88);25OHvitamineD:29,73ng $/ \mathrm{mL}(\mathrm{RV}: 11-70)$; estradiol $45 \mathrm{pg} / \mathrm{ml} ; \mathrm{FSH}:<0.2 \mathrm{mU} / \mathrm{ml} ; \mathrm{LH}$ $<0.1 \mathrm{mu} / \mathrm{ml}$. A stimulating mutation of GNAS gene was identified.

Case 3: 3.7 years old tall, overweighted girl evaluated for unilateral breast budding and two episodes of vaginal spotting. Bone age was of 5 years. Laboratory evaluation showed high levels of estradiol with a pre pubertal response on LHRH stimulation test; cintigraphy showed a very large occipital dysplasic area. She has had several episodes of vaginal bleeding of short duration. At 9 years of age (BA 12 years), breast increased as well pubic and axillar hair; at 9.5 years vaginal bleeding became regular.

Conclusion: The possibility of MAS should be remembered in patients with precocious vaginal bleeding, "café au lait" maculas or bone lesions. Clinic presentation, severity and progression are variable.

\section{EPO-236 Puberty and Neuroendocrinology \\ Similarities and differences between central precocious puberty with first sign was pubarche or thelarche \\ Caroline Goncalves; Mariana Sarti de Paula; Soraya L S Milani; \\ Patricia V S Atique; Rodrigo Custodio; Ana Claudia Reis; \\ Marcelo Ruiz; Sonir Antonini; Raphael Liberatore Jr; \\ Carlos Martinelli Jr \\ School of Medicine of Ribeirão Preto, Paediatrics, Ribeirão Preto, Brazil}

Background: Little data are available regarding girls with central precocious puberty $(\mathrm{CPP})$ who start clinical manifestation with premature pubarche (PP). Objective: To compare clinical and laboratory data of girls who started CPP with PP and girls which first sign of CPP was premature thelarche (PT).

Methods: Fifty girls with CPP was enrolled and grouped according to the first pubertal sign: pubarche (group GPP, $\mathrm{n}=25$ ) or thelarche (group GPT, $\mathrm{n}=25$ ). Information regarding chronological age (CA) at the appearance of the first pubertal sign (iCA), time-interval (Ti) between pubarche and thelarche, bone age (BA), LH and FSH levels were obtained from medical records.

Results: iCA were lower in GPT $(4.8 \pm 2.4 \mathrm{yrs})$ than in GPP $(6.1 \pm 1.0 \mathrm{yrs}$, $\mathrm{P}=0.01)$. BA at the first visit $(9.2 \pm 1.5 \mathrm{yrs})$ were $7.0 \pm 1.0$ months higher than $\mathrm{CA}(\mathrm{P}=0.0001)$ in GPP and 6.6 \pm 2.2 months higher than CA in GPT $(8.0 \pm 2.9$ years; $\mathrm{P}=0.07$ ). Ti between pubarche and thelarche was $18.4 \pm 14.3$ months in GPP while between thelarche and pubarche was $20.1 \pm 15.7$ months in GPT $(\mathrm{P}=0.8)$. Ti between the first sign and the diagnosis of CPP was similar in GPP ( $0.8 \pm 0.6$ months) and GPT ( $1.1 \pm 1.4$ months; $\mathrm{P}=0.9)$.

No difference was observed regarding FSH or LH levels at first visit between GPP and GPT (FSH: GPP median $1.9 \mathrm{mU} / \mathrm{ml}$, range: $0.2-9.0 \mathrm{mU} / \mathrm{ml}$; GPT mean $2.4 \mathrm{mu} / \mathrm{ml}, 0.4-10.8 \mathrm{mU} / \mathrm{ml}$; P=0.6) (LH: GPP median $0.24 \mathrm{mU} / \mathrm{ml}, 0.1$ $6.5 \mathrm{mU} / \mathrm{ml}$; GPT mean $0.45 \mathrm{mU} / \mathrm{ml}, 0.1-6.9 \mathrm{mU} / \mathrm{ml} ; \mathrm{P}=0.6)$. LH peak $(\mathrm{GnRH}$ test) were also similar in GPP (median $6.5 \mathrm{mU} / \mathrm{ml}, 0.3-44.2 \mathrm{mU} / \mathrm{ml}$ ) and in GPT (mean $9.1 \mathrm{mU} / \mathrm{ml}, 0.24-58.1 \mathrm{mU} / \mathrm{ml} ; \mathrm{P}=0.4$ ).
Conclusion: The interval between the two main characteristics of puberty, pubarche and thelarche were similar no matter which of them came first. Although PT is an earlier manifestation of CPP than PP, clinical, laboratory and radiologic parameters are very similar in both situations of CPP opening.

\section{EPO-237 Puberty and Neuroendocrinology \\ Neuroprotective and antiaging roles of dehydroepiandrosterone in aging male rat brain Pardeep Kumar; $R$ K Kale; N Z Baquer}

Jawaharlal Nehru University, School of Life Sciences, New Delhi, India

Introduction: Dehydroepiandrosterone (DHEA), one of the major steroid hormones, and its ester have recently received attention with regard to aging and age-related diseases like Alzheimer 's disease. These changes increase during aging when the level of DHEA is decreased. DHEA is synthesized de novo in the brain and its substantial fall with age has been shown to be associated with neuronal vulnerability to neurotoxicity processes.

Objective: The objective of this study was to observe the changes in activitiy of monoamine oxidase (MAO), glucose transporter-4 levels (GLUT-4), membrane fluidity, lipid peroxidation levels and neurolipofuscin accumulation occurring in aging male rat brain, and to see whether these changes are restored to normal levels after exogenous administration of DHEA.

Methods: The aged male rats (4,14 and 24 months age group) were given subcutaneous injection of DHEA ( $30 \mathrm{mg} / \mathrm{kg} /$ day for 1 month). Results: The results obtained in the present work revealed that normal aging was associated with significant increases in the activity of MAO, lipid peroxidation levels and neurolipofuscin accumulation in the brains 4, 14 and 24 months age group male rats, and a decrease in GLUT-4 and membrane fluidity. Administration of DHEA brought these changes to near normalcy.

Conclusions: It can therefore be suggested that DHEA's beneficial effects seemed to arise from its antioxidant, antilipofuscin, antilipidperoxidative and thereby neuroprotective actions.

\section{EPO-238 Sex Differentiation \\ Meacham syndrome with cardiac defect is not due to WT1 mutation}

Karine Gillieron; Monika Meyer; Eugen Schoenle; Anna Biason-Lauber University Children's Hospital, Endocrinology and Diabetology, Zurich, Switzerland

Background: Meacham syndrome is a rare and sporadically occurring multiple malformation syndrome, characterized by congenital diaphragmatic abnormalities, cardiac and pulmonary malformations, $46 \mathrm{XY}$ disorder of sex development (DSD) and consequent early infant death. Since WT1 knock-out mice have a phenotype similar to Meacham syndrome (they lack kidneys, spleen, gonads and adrenals, have a thin ventricular myocardium and die rapidly of cardiac failure), WT1 mutations have been sought and found in some patients affected by this disease.

Case report: A $46 \mathrm{XY}$ baby with a complete female phenotype developed respiratory distress syndrome right after birth, leading to the diagnosis of congenital heart malformation, hypoplastic right lung and right diaphragmatic hernia. Furthermore, a true double vagina with uterus didelphis was found in the presence of external female genitalia with clitoris hypertrophy and small vaginal ostium. MRI showed no evidence of gonads. Respiratory deterioration led to death on the $130^{\text {th }}$ day of life. Given the identity of this phenotype to classical Meacham syndrome, we screened the entire coding sequence (exons 1-10) and intron-exon boundaries of $W T 1$ for mutations. No mutation could be found.

Conclusions: In spite of the clinical picture apparently identical to the originally described Meacham syndrome, the presence of congenital heart defects associated with the typical 46 XY DSD with pulmonary, diaphragmatic and genital anomalies, might indicate a clinical entity distinct from Meacham syndrome and mutations in genes other than WTI should be considered as the molecular basis of the disease. 


\section{EPO-239 Sex Differentiation}

46, XY disorders of sexual development (DSD) presenting with normal female genital phenotype: Leydig cell hypoplasia or aplasia Enver Simsek ${ }^{1}$; Cigdem Binay'; Sare Kabukcuoglu; Baran Tokar ${ }^{3}$ ${ }^{1}$ Eskisehir Osmangazi University School of Medicine, Pediatric Endocrinology, Eskisehir, Turkey; ${ }^{2}$ Eskisehir Osmangazi University School of Medicine, Pathology, Eskisehir, Turkey; ${ }^{3}$ Eskisehir

Osmangazi University School of Medicine, Pediatric Surgery, Eskisehir, Turkey

Introduction: Leydig cell hypoplasia or aplasia is rare one of the etiologies of 46, XY DSD. This is an autosomal recessive disease and caused by LHreceptor inactivation mutation. This disease is characterized by rudimenter Wolffian duct, female genitalia phenotype, and low level of basal and hCG stimulated testosterone.

Case report: 22- month-old girl presented with swelling in the inguinal region. Abdomino-pelvic ultrasound examination revealed no mullerian structure and bilateral inguinal mass which were similar to testis (Fig.1). She was referred to endocrine department for investigation of the etiology of DSD. She was the second child of first degree consanguineous parents. Physical examination revealed normal female genital phenotype (Fig.2), no genital pigmentation, phallus size $0.3 \mathrm{~cm}$, no labiscrotal fusion, and bilateral palpable mass in inguinal channels. FISH analysis revealed 46, XY karyotype and positive SRY. Endocrinological investigation revealed that basal and hCG stimulated testosterone levels were undetectable, antimullerian hormone level was $175 \mathrm{ng} / \mathrm{ml}$ (normal reference for male infant, 32-180 ng/ml). Bilateral gonadal biopsy revealed Leydig cell agenesis (Fig.3, Fig.4). DNA sample was sent for the mutation analysis of Leydig hormone receptor (waiting the result). The family was informed about the disease. Bilateral gonadectomy was performed based on parental consent.

Conclusion: If a 46, XY DSD presented with female genital phenotype, without fluid and electrolyte imbalances, low basal and hCG stimulated testosterone levels, and positive SRY anlysis, the possible diagnosis is Leydig cell hypoplasia or agenesis. Histological investigation of palpable gonads confirms the diagnosis. If possible, genetic study for mutation analysis of $\mathrm{LH}$ receptor gives additional information. Nearly all of the families prefer female identity after detailed information.
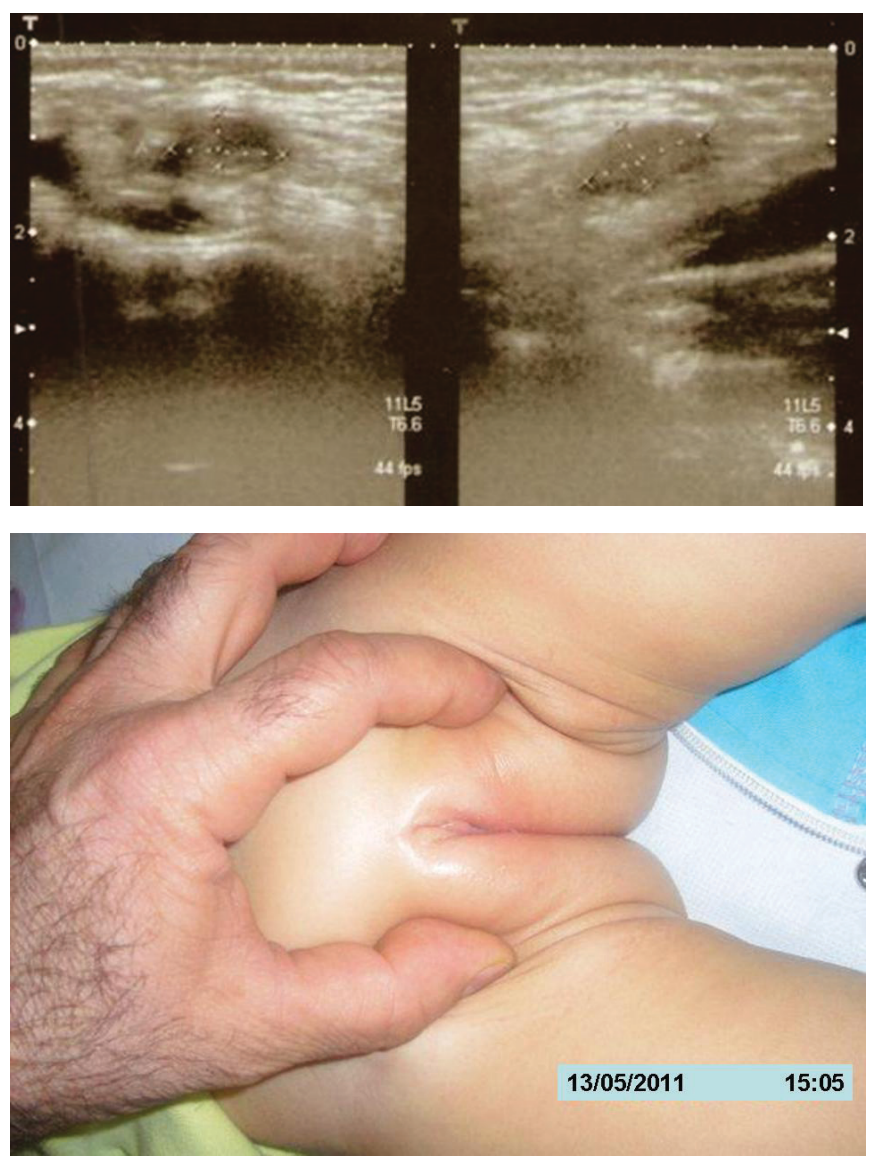
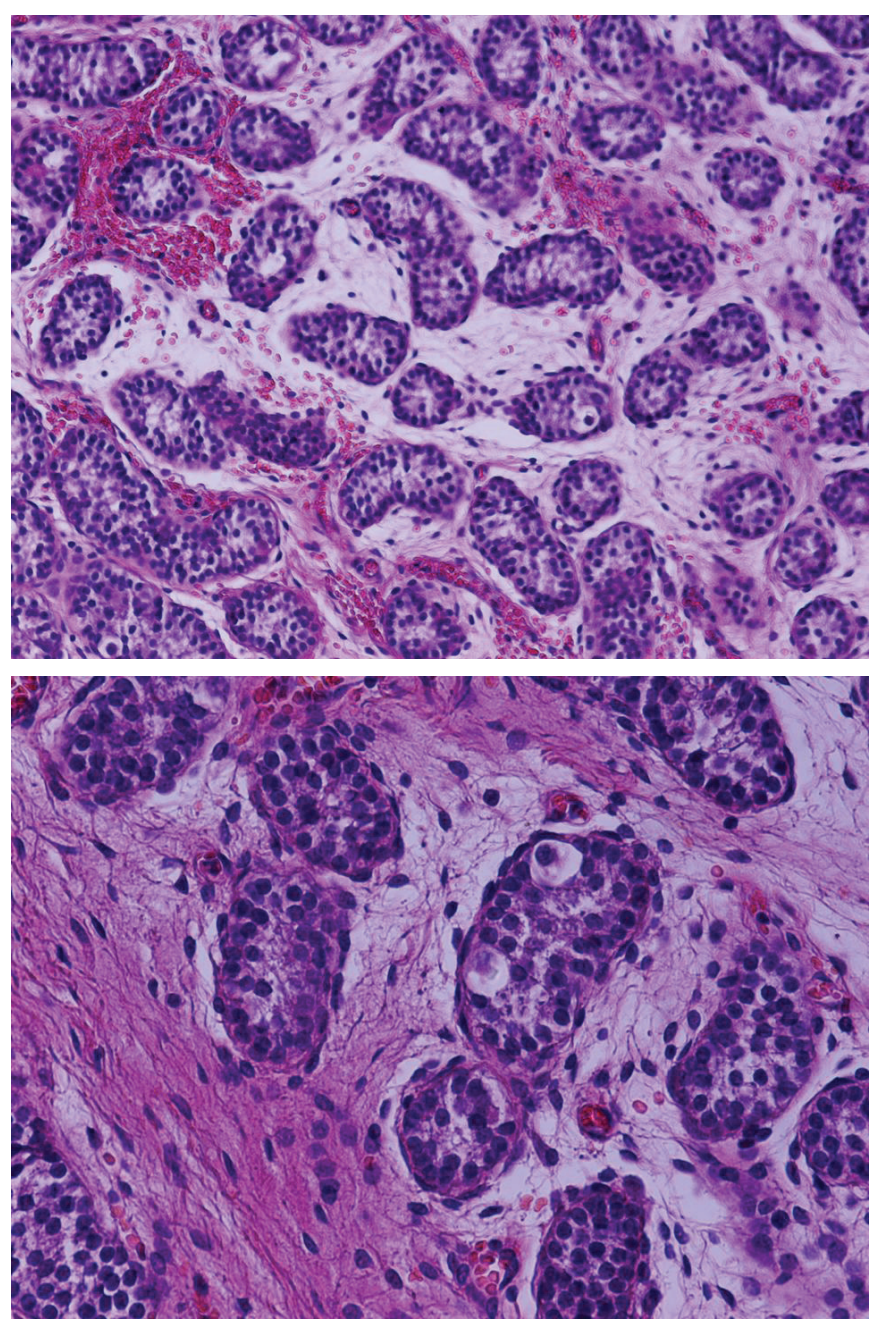

EPO-240 Sex Differentiation

\section{Healthcare for children with disorders of sex development in a developing country}

Adela Chirita Emandi' ; Monica Marazan'; Corina Pienar'; Maria Puiü; loana Micle ${ }^{1}$

'Emergency Hospital for Children "Louis Turcanu", Endocrinology, Timisoara, Romania; ${ }^{2}$ University of Medicine and Pharmacy "Victor Babes", Endocrinology, Timisoara, Romania; ${ }^{3}$ University of Medicine and Pharmacy "Victor Babes", Genetics, Timisoara, Romania

Background: Disorders of sex development (DSD) is a relatively new concept and guidelines are currently still in development. Assessing gender in a child with DSD is a sensitive issue that requires a multidisciplinary team expertise, both in the initial approach and ongoing management.

Objective and hypotheses: To evaluate current management of children with DSD in a tertiary pediatric hospital in western Romania.

Methods: We performed a retrospective analysis of medical charts of 56 children with DSD, admitted between 2008-2011.

Results: Median age 11+/-6.1 years; 36 children were diagnosed with congenital adrenal hyperplasia (karyotype: 26 patients-46XX; 10 patients-46XY; 2 patients had wrong sex assignment at birth and were reassigned later); 16 children with Turner syndrome (13 patients-45X; 3 patients-45X/46XX); 1 patient with mixed gonadal dysgenesys $(45 \mathrm{X} / 46 \mathrm{XY}), 1$ child with Klinefelter syndrome $(45 \mathrm{X} / 46 \mathrm{XY}) ; 1$ child with POR gene deficiency (46XX- declared boy at birth and reassigned at 5 months), 1 child with partial androgen insensivity (46XY). We encountered several difficulties in management of some children with DSD: decreased awareness of DSD in medical practitioners, limited access to some diagnostic tests; lack of clear guidelines for indication and timing of genital surgery, paucity of surgeons with specific expertise in the care of children with DSD, fear of legal implication for surgeons. Also, we 
found absence of local guidelines of management. Although intense efforts are made to create European guidelines (EuroDSD), they might be difficult to adapt for Romania, due to cultural differences and psycho-social support structures dissimilarities, between developed and developing countries.

Conclusions: Psychosocial issues related to gender identity in some children with DSD are very sensitive and require an individual approach; moreover there is an urgent need for creation of national reference centers and guidelines for management of DSD.

\section{EPO-241 Sex Differentiation \\ Normal value of anti-mullerian hormone (AMH) in Thai children and its application to diagnose patients with 46,XY DSD \\ Vichit Supornsilchai; Rottanat Rugtolmuang; \\ Taninee Sahakitrungruang; Suphab Aroonparkmongkol; \\ Suttipong Wacharasindhu \\ Chulalongkorn University, Pediatrics, Bangkok, Thailand}

Background: Anti-Mullerian hormone (AMH), $140 \mathrm{kDa}$ glycoprotein hormone belonging to the TGF- $\beta$ superfamily, is a testis specific marker. AMH is mainly produced by sertoli cell but, it is minimally produced by the ovaries. There is no extragonadal source of AMH. The levels of AMH are varied by ages and influenced by many hormones and transcription factors. The stimulation factors are SOX-9, SF1, GATA4 and WT1 and the inhibition factors are androgens and meiotic germ cells. There are previous studies showing normal value of AMH in boys:

\begin{tabular}{lcccc}
$\begin{array}{l}\text { Age/ genital } \\
\text { Tanner }\end{array}$ & $\begin{array}{c}\text { AMH (pmol/L), } \\
\text { median (5-95\%) } \\
\text { (Aksglade, 2011) }\end{array}$ & $\begin{array}{c}\text { AMH (pmol/L), } \\
\text { mean SD (Rey, } \\
\text { 2005) }\end{array}$ & $\begin{array}{c}\text { AMH (pmol/L), } \\
\text { mean SD } \\
\text { (R.P.Grinspon } \\
\text { 2011) }\end{array}$ & $\begin{array}{c}\text { AMH (pmol/L), } \\
\text { mean SD (our } \\
\text { study) }\end{array}$ \\
\hline 0-1 mo & $148(53-340)$ & $523 \pm 156$ & $621 \pm 271$ & $987.8 \pm 193.9$ \\
\hline 1-4 mo & 1047 (749-1930) & $699 \pm 245$ & $761 \pm 357$ & $995.3 \pm 180.7$ \\
\hline 4-12 mo & $1082(395-1397)$ & & & $1020 \pm 142.8$ \\
1-2 yr & $755(395-1335)$ & $793 \pm 264$ & $1253 \pm 502$ & $907.46 \pm 251.7$ \\
\hline 2-4 yr & & & $782 \pm 461$ & $890.5 \pm 230.1$ \\
4-6 yr & & & & $781.9 \pm 247.3$ \\
6-8 yr & & $516 \pm 275$ & & \\
8-10 yr & $697(321-1218)$ & & $741 \pm 327$ & $742.3 \pm 250.2$ \\
G II & $253(46-1120)$ & $249 \pm 126$ & $419 \pm 301$ & $239.1 \pm 354.6$ \\
G III & $66(22-734)$ & $98 \pm 65$ & $121 \pm 114$ & $65 \pm 31.5$ \\
\hline G IV $N$ & $49(23-128)$ & $42 \pm 38$ & $84 \pm 45$ & $117.6 \pm 60.9$
\end{tabular}

Many applications of AMH are purposed to diagnose many conditions i.e. DSD, nonpalpable gonad, hypogonadotropic hypogonadiam and precocious puberty.

Objective: To define the levels of AMH in normal control as the following ages, 0-1 mo, 1-3 mo, 3-12 mo, 1-2 yr, 2-4 yr 4-8 yr, 8-12 yr, genial Tanner II, III, IV and V and in $46 \mathrm{XY}$ DSD for determining the cause.

Methods: Control are divided in different age groups as mention and the clinical data including age at sample collection, genital Tanner. The numbers of each group is calculated by the formula as in the previous study. The AMH level is analysed by ELISA technique and LH, FSH and testosterone are analysed by chemiluminesceent assay.

Results: The AMH levels and its trends at different ages are correlated with the previous reports (table1, figure 1a). The normal value with ages of FSH, $\mathrm{LH}$ and testosterone are shown in figure 1b. The AMH levels of 46,XY DSD in our study are shown in

$\begin{array}{lcccc}\text { Diagnosis } & \mathbf{n} & <\mathbf{1 S D} & \text { mean } & >1 \text { SD } \\ \text { PAIS (confirmed AR mutation) } & 1 & 1 & - & - \\ 46, \text { XY DSD (no AR mutation) } & 3 & - & 2 & 1 \\ 46, \text { XY DSD (unknown cause) } & 10 & 2 & 5 & 3 \\ 46 \text { XX male } & 1 & 1 & - & - \\ \text { MGD } & 1 & - & 1 & -\end{array}$

which are mainly normal for ages.

Conclusions: The AMH level in the present study can be represent as the normal value for Thai children and can initially define the causes of 46,XY DSD.
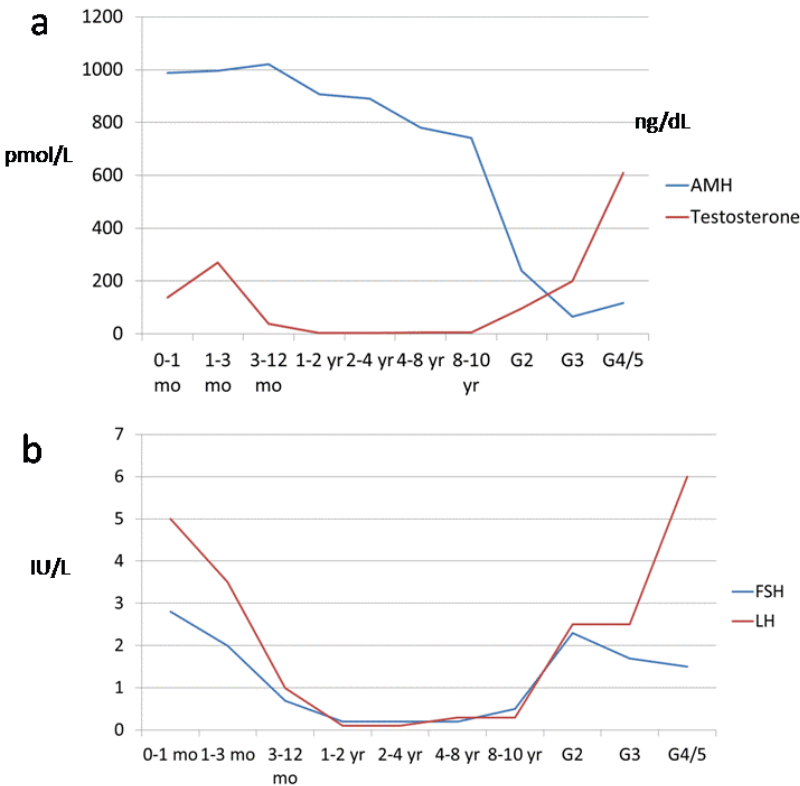

\section{EPO-242 Sex Differentiation \\ Two cases with Complete Androgen Insensitivity Syndrome and Discordant Müllerian remnants}

Ayla Güven'; Fatma Dursun'; Çigdem Ulukaya Durakbapa²; Peyma Özkanli ; Berrin Güçlüer ${ }^{3}$; Ihsan Kuru ${ }^{4}$

${ }^{1}$ Göztepe Training and Research Hospital, Pediatric Endocrinology, Istanbul, Turkey; ${ }^{2}$ Göztepe Training and Research Hospital, Pediatric Surgery, Istanbul, Turkey; ${ }^{3}$ Göztepe Training and Research Hospital, Pathology, Istanbul, Turkey; ${ }^{4}$ Göztepe Training and Research Hospital, Radiology, Istanbul, Turkey

Introduction: Complete Androgen Insensitivity Syndrome (CAIS) is an Xlinked disorder characterized by end-organ resistance to testosterone and dihydrotestosterone (DHT). It typically results from mutations in the androgen receptor (AR) gene in patients with typical male 46, XY karyotypes. The Müllerian system usually regresses because of the presence of the anti-Müllerian hormone (AMH) originating from the sertoli cells of the gonads. Occasionally residual Müllerian structures exist. We present a case of CAIS in two members of the same family, reared as females but found to have a 46, XY genotype, with residual Müllerian tissue.

\begin{tabular}{lcc} 
& Patient 1 & Patient 2 \\
\hline Body weight $(\mathrm{kg})$ & $58(90$ th $\mathrm{p})$ & $68(90-95$ th $\mathrm{p})$ \\
\hline Height $(\mathrm{cm})$ & $163(90$ thp $)$ & $165.5(50-75$ th $\mathrm{p})$ \\
\hline Pubic hair $($ Tanner $)$ & Stage 2 & Stage 1 \\
\hline Breast $($ Tanner) & Stage 5 & Stage 5 \\
\hline $\mathrm{LH}(\mathrm{mlU} / \mathrm{ml})$ & 43.7 & 37 \\
$\mathrm{FSH}(\mathrm{mlU} / \mathrm{ml})$ & 18.6 & 11 \\
\hline Estradiol $(\mathrm{ng} / \mathrm{ml})$ & 22 & 22 \\
\hline Testosterone $(\mathrm{ng} / \mathrm{ml})$ & 8.66 & 2.42 \\
\hline AMH $(\mathrm{ng} / \mathrm{ml})$ & 29.25 & 24.25 \\
Pelvic US & Uterus-like appearing tissue, & Uterus-like appearing tissue, \\
ovaries were not visible & ovaries were not visible \\
Karyotype & $46 \mathrm{XY}$ & $46 \mathrm{XY}$
\end{tabular}

Case 1: A twelve year-old-girl, presented with polidipsi and hyperglicemia (blood glucose: $587 \mathrm{mg} / \mathrm{dl}$ ) was diagnosed as type 1 diabetes mellitus. She was born from nonconsanguineous parents after a full-term pregnancy. She had been operated because of inguinal hernia when she was 7 year old. Her three aunts and one cousin had never menstruated which led us suspect from CAIS and investigate the patient (Table).

Case 2: 18 year old girl, cousin of the first patient was investigated because 
of primary amenorrhoea and history of inguinal hernia operation (Table). Patients underwent laporoscopic bilateral gonadectomy. Histopathology revealed leydig cell hyperplasia and only sertoli cells in seminifer tubules. In one side of the testes, hypoplastic tuba and hypertrophic muscle which were positively stained with estrogen and progesteron were present. The histopathologic findings of the other patient was similar. Mutation analyse of the cases are pending.

Conclusion: An unusual variety of CAIS in two cousins is presented.

\section{EPO-243 Sex Differentiation}

\section{Unfeasibility of surgically separating male and} female components of an ovotestis gonad: a case report

\section{Philippe Lysy'; François-Xavier Wese' ${ }^{2}$; Pascale Jadouß ${ }^{\text {; }}$ Marc Maes ${ }^{1}$}

${ }^{1}$ Cliniques Universitaires Saint Luc, Université Catholique de Louvain,

Pediatric Endocrinology, Brussels, Belgium; ${ }^{2}$ Cliniques Universitaires

Saint Luc, Université Catholique de Louvain, Pediatric Urology,

Brussels, Belgium; ${ }^{3}$ Cliniques Universitaires Saint Luc, Université

Catholique de Louvain, Gynecology, Brussels, Belgium

Background: Ovotestis is associated with a malignancy risk of 3\% according to currently available data. The risk of tumor formation is correlated with the presence of immature female or male gonadal tissue in the ovotestis. The macroscopic aspect of the ovotestis is either undifferentiated or differentiated into ovarian and testicular tissues. In the latter situation, it is currently recommended to remove the testicular tissue if the patient is raised as a female. However, the feasibility of separating ovarian and testicular tissues of an ovotestis gonad is not well known.

Objective and hypotheses: Based on a clinical case, to retrospectively evaluate the feasibility of a surgical dissociation of the male and female components of an ovotestis gonad.

Methods: Our patient presented at birth with a disorder of sexual development including a chordee, a second degree hypospadias, a left undescended and a right palpable gonad. The karyotype was $46, \mathrm{XX}$ and the parents decided to raise the child as a female. The patient underwent feminizing genitoplasty at 1 month and during the procedure, the component of both gonads corresponding macroscopically to testicular tissue was excised while the ovarian component was left in place. Diagnosis of ovotestis was confirmed by histology of the excised tissues. Female pubertal development occurred normally. Results: The patient presented at 22 years with abdominal pain. Ultrasound showed a heterogeneous pelvic mass of $9 \mathrm{~cm}$ originating from the left ovary. The biopsy performed during laparoscopy showed a mixed germinal tumor containing a yolk sac tumor and a gonadoblastoma. The patient underwent excision of the tumor and of the right ovary (containing primary follicles and testicular tissue), followed by chemotherapy.

Conclusions: Our case illustrates the unfeasibility of surgically removing the testicular component of an ovotestis in a female patient and emphasizes the tumor risk when dysgenetic gonads are left in place.

\section{EP0-244 Sex Differentiation \\ 46XY disorders of sexual development: an overview in a specialized endocrine centre in Egypt \\ Isis Ghaly'; Mona Hafez²; Abeer Atef Alashmawy²; Mona Mamdouh²;} Sheriene Abdel-Ghaffar²; Nora Badawi2; Amany Ibrahim²; Hend Mehaoed ${ }^{2}$

${ }^{1}$ Kasr Alaini, Faculty of medicine, Cairo University, Diabetes, Endocrine and Metabolism Pediatric Unit (DEMPU), Giza, Egypt; ${ }^{2}$ Kasr Alaini, Faculty of medicine, Cairo University, Diabetes, Endocrine and Metabolism Pediatric Unit (DEMPU), Cairo, Egypt

Background: Disorders of sex development (DSD) represents a group of heterogeneous etiopathogenesis and clinical manifestations, it is important that a definitive diagnosis be determined as quickly as possible so that the appropriate treatment plan can be established to minimize medical, psychological and social complications.

Objective and hypotheses: To provide a detailed review of the clinical characteristics as well as hormonal aspects of Egyptian children with 46 XY DSD presenting to the Diabetes, Endocrine and Metabolic Pediatric Unit (DEMPU) and to show the great magnitude of DSD in Egypt
Patients: Two hundreds and fifty patients aged from newborn to 19 years with 46 XYDSD.

Methods: All cases were subjected to history taking. Full clinical and genital examination, hormonal, and radiological investigations were performed.

Results: (59.38\%) had androgen action disorder, (31.25\%) testosterone biosynthesis defect and (9.37\%) disorders of testicular development. There was preference of male sex of rearing, despite a severe degree of ambiguity. The sex of rearing was incorrectly female in $37.5 \%$ of cases and male in $62.5 \%$ of cases.

Conclusions: There are many difficult aspects in the diagnosis and management of46 XY DSD. Gender assessment teams of endocrine centers need a multidisciplinary approach for the diagnosis, medical and surgical treatment, genetic counseling, and psychosocial support. Molecular analysis for cases with 46, XY DSD is essential for accurate diagnosis and proper gender assignment.

\section{EPO-245 Sex Differentiation}

\section{Adolescent with disorder of sex development due to 17- $\beta$-hydroxysteroid-dehydrogenase-3 enzyme deficiency by mutation p.Glu215Asp on the HSD17B3 gene}

\section{Carla Costa'; Cíntia Castro-Correia'; Sofia Martins ${ }^{2}$.}

Alda Mira-Coelho; ${ }^{3}$ Bessa Monteiro ; Joaquim Monteiro ${ }^{4}$; leuan Hughes ${ }^{5}$; Manuel Fontoura ${ }^{1}$

${ }^{1}$ Hospital São João, Paediatric/ Paediatric Endocrinology Unit / Faculty of Medicine of Porto, Porto, Portugal; ' ${ }^{2}$ ospital São João, Paediatric/

Paediatric Endocrinology Unit, Porto, Portugal; ${ }^{3}$ Hospital São João, Psychiatry, Porto, Portugal; ${ }^{4}$ Hospital São João, Paediatric Surgery, Porto, Portugal; ${ }^{5}$ Hospital Addenbrooks, Paediactric/ Paediatric Endocrinology Unit/ University of Cambridge, Cambridge, United Kingdom

Background: The development of male internal and external genitalia in an $\mathrm{XY}$ fetus requires a complex interplay of many critical genes, enzymes and cofactors. The enzyme 17 $\beta$-hydroxysteroid-dehydrogenase type-3 (17HSD-3) is present almost exclusively in the testicles and converts $\Delta 4$-androstenodione (A) to testosterone (T). A deficiency in this enzyme is rare and frequently misdiagnosed autosomal recessive cause of 46XY, DSD. If not diagnosed early, patients present a severe virilization and primary amenorrhea at puberty and may undergo a change from female to male gender.

Clinical case: Fifteen year old adolescent, was raised accordingly to female gender. At puberty, refers a severe virilization and primary amenorrhea. Physical examination: male phenotype with micropenis and blind vagina. Tanner stage: A3B1P4, non palpable gonads. The karyotype revealed 46XY. Endocrinology study: $\mathrm{T}=2.38 \mathrm{ng} / \mathrm{ml} ; \mathrm{A}>10.00 \mathrm{ng} / \mathrm{ml}$; low $\mathrm{T} / \mathrm{A}$ ratio=0.23; $\mathrm{DHT}=$ $0.127 \mathrm{ng} / \mathrm{ml}$; T/DHTratio=18.7 and urinary steroids: normal. Abdominal-pelvic MRI: presence of testicles in inguinal canal, seminal vesicle, prostate, micropenis and absence of uterus and vagina. Started treatment with GnRH analogue and flutamide. The genetic study confirmed the mutation p.Glu215Asp on HSD17B3 gene in homozygosity. During all procedures was accompanied by a child psychiatrist/psychologist. The teenager wants to be female, so gonadectomy was performed and started estrogens therapy and the first steps to surgical procedure to change external genitalia.

Conclusions: In literature, 27 mutations in HSD17B3 gene have been reported. The mutation p.Glu215Asp was already found in three Childs with 46XY, DSD. Correct diagnosis should be made early so that treatment, management and genetic counseling can be specifically directed towards 17HSD-3 enzyme deficiency. In this case, there was a severe virilization at puberty. It's speculated to be due to a partial activity of 17HSD-3 in the testicles and/or extratesticular ability to convert androstenodione to testosterone by 17HSD-5. 
EPO-246 Sex Differentiation

The decision-making process in a case of late diagnosed 5alpha-reductase deficiency

Wiebke Birnbaum; Louise Marshall; Olaf Hiort

University of Lübeck, Pediatric Endocrinology, Lübeck, Germany

Background: There is a lack of standardization of medical consult within the field of DSD. New recommendations from ethical stakeholders have to be considered.

Objective and hypotheses: We describe a case of 5alpha-reductase deficiency (5-ARD) as an example for a complex decision-making process. Our multidisciplinary team (MDT) was consulted to lead the decision-making process. What are the individually tailored steps to ensure a satisfying longterm outcome?

Methods: We report on a Kurdish 46, XY girl not diagnosed with 5-ARD until the age of $131 / 2$ years and sketch the time-line and the steps which contribute to the informed medical consent. Diagnostic evidence was achieved by molecular genetic analysis. We explained 5-ARD and the specific implications to the patient and the family separately attended by a translator. The psychologist assessed gender behaviour and identity. Information from the local child protective service completed the picture on the area of conflict.

Results: All involved parties shared facts, treatment options and possible outcomes. The "no treatment" option would lead to an irreversible decision.(Progressive virilisation/ gender reassignment). The female line would result in gonadectomy and hormone replacement therapy. The profound consequences for the patient, her immaturity and the fathers ambiguity forbid a finalizing decision at this stage. Hormone suppression allowed a limited moratorium to further evaluate gender identity and to empower the patient to consent. A re-evaluation after six months (June 2012) shall lay the basis for the ongoing treatment.

Conclusions: The management of patients with DSD requires care through specialised multidisciplinary centres which analyses the different options and perspectives. This decision takes time and is straining. Disclosure process and informed consent management need to be standardised in consideration of patient centred care. Following these guidelines a greater compliance with ethical standards will be achieved.

\section{EP0-247 Sex Differentiation \\ Klinefelter's syndrome phenotype in a boy with 46, dupX (p22.2; 22.3)Y/46, XY mosaicism \\ Vilhelm Mladenov ${ }^{1}$; Violeta lotova ${ }^{1}$; Lyudmila Angelova ${ }^{2}$ \\ 1 UMHAT "Sveta Marina", Pediatrics, Varna, Bulgaria; 'UMHAT "Sveta \\ Marina”, Genetics, Varna, Bulgaria}

Background: Different aberrations or rearrangements of the X chromosome are often found in male patients with various degrees of mental retardation or behavioral problems.

Objective and hypotheses: We hypothesize that even duplications of parts of the X chromosome could lead to typical Klinefelter's syndrome.

Methods: We present an 11 years 6 months old boy born after uneventful pregnancy and delivery of a 35 years old mother and 41 years old father. Weight at birth was $3.700 \mathrm{~kg}$, length $-55 \mathrm{~cm}$. After infancy a developmental delay and language difficulties were noticed, and after 2 years of age he began to gain weight excessively. At the age of 9 years because of mild mental retardation, hyperactivity and a phenotype suspicious of fragile $\mathrm{X}$ syndrome a cytogenetic testing was performed for exclusion of the latter.

Results: The result was mosaicism 46, dupX (p22.2-22.3)Y/46, XY in $81 \%$ of the cells. At present the patient has moved towards a typical Klinefelter's syndrome appearance with tall stature out of the target range, obesity, normal testicular volume - $4 \mathrm{ml}$, micropenis $-35 \mathrm{~mm}(>-2.5 \mathrm{SD})$ and gynecomastia. The hormonal levels are adequate for the beginning of puberty - LH 0.365 $\mathrm{mIU} / \mathrm{ml}$, FSH $2.42 \mathrm{mIU} / \mathrm{ml}$, Testosteron < 0,69 nmol/1, Estradiol $83.3 \mathrm{pmol}$ and the bone age is slightly advanced -13 years.

Conclusions: We conclude that even duplications of parts of the X chromosome could lead to typical Klinefelter's syndrome. Our patient's genetic abnormality is consistent predominantly with mental delay and is rarely reported in connection with Klinefelter's syndrome. This warrants karyotyping even in patients with mild Klinefelter's syndrome phenotype whenever considerable mental delay is present.

51st Annual Meeting of the ESPE
EPO-248 Sex Differentiation

A patient with a rare $46, X X / 47, X X Y$ karyotype: a new unique case and review of the literature

Natallia Akulevich; Julia Boiko; Yuliya Makarava

State Center of Medical Rehabilitation, Pediatrics, Pediatric

Endocrinology Group, Minsk, Belarus

Background: True hermaphroditism is a rare condition characterized by the presence of both ovarian and testicular tissue, with the most common 46,XX karyotype. Klinefelter syndrome is a common type of human sex chromosome abnormalities, typically with 47,XXY karyotype.

Objective and hypotheses: To describe a DSD in a child with male phenotype and a rare 46,XX/47,XXY mosaic karyotype.

Methods: Patient's early history, clinical and laboratory evaluation, histological and cytogenetic studies, comparative literature review.

Results: The child had ambigous genitalia since birth: unilateral criptorchidism on the right and perineal hypospadia. At the age of 1.5 months he underwent surgery for the strangulated right inguinal hernia: fallopian tube and a necroticised right gonad were removed. Histopathological examination revealed ovarian tissue. Cytogenetic analysis of peripheric blood at the age of 1.5 years detected 46,XX $(80 \%) / 47, \mathrm{XXY}(20 \%)$ karyotype. Hypospadia was repaired. The Mullerian remnants were removed laparoscopically also on the left. At the age of $12 \mathrm{yrs}$ the patient was referred to our clinic. His stature was - 0,2 SDS, weight - 1 SDS, bone age: 13 yrs, pubertal stage: Px3Ax1; left testis size was $1 \mathrm{ml}$, bilateral ginecomastia was present. Both serum LH and FSH levels were evevated, basal testosterone and estradiol were low. At ultrasound, a structure typical for testicular tissue on the left and the rudimentary uterus were present. Surgical removal of any Mullerian structures left was recommended. Testosterone replacement therapy for primary hypogonadism has been prescribed. The left gonade, presumably testis, requires biopsy and further monitoring for malignancy.

Conclusions: This case of true hermaphroditism with male phenotype and 46,XX/47,XXY Klinefelter syndrome's mosaic karyotype is intriguing for finding of Mullerian remnants on both sites. A comparative review of reported cases will be done to stress different aspects of follow-up to every such a patient.

\section{EP0-249 Sex Differentiation \\ Severe virilisation of a $46, X X$ newborn and her mother during pregnancy: a novel case of aromatase deficiency}

Stefan Riedl'; Alexander Springer ${ }^{2}$; Johannes Huber ${ }^{3}$

'St Anna Children's Hospital, Medical University of Vienna, Pediatric Department, Vienna, Austria; ${ }^{2}$ Medical University of Vienna,

Department of Pediatric Surgery, Vienna, Austria; ${ }^{3}$ Medical University of Vienna, Department of Obstetrics and Gynecology, Vienna, Austria

Background: Aromatase (CYP19A1) deficiency is a very rare cause of 46,XX DSD. Excess of fetal androgens due to lack of placental aromatisation leads to virilisation of external genitalia and maternal virilisation during pregnancy.

Patients and results: We present a female newborn with Prader stage 4-virilized genitalia, 3rd child from Turkish consanguineous parents. Starting from the second trimester, the mother had developed progressive hirsutism, acne, and voice deepening. At presentation on day 12 post-partum, her LH and FSH were suppressed $(<0.1 \mathrm{mU} / \mathrm{ml})$ in the presence of extremely high testosterone $(\mathrm{T} ; 10.4 \mathrm{ng} / \mathrm{ml})$, androstendione $(\mathrm{A} ; 69.4 \mathrm{ng} / \mathrm{ml})$, 17-hydroxy progesterone (17-OHP; $11.4 \mathrm{ng} / \mathrm{ml})$, and estrogen (E2; $2.787 \mathrm{pg} / \mathrm{ml})$. Sonography revealed markedly enlarged ovaries with multiple cysts. An adrenal/ovarian virilising tumour was ruled out by negative tumour markers and MRI. By day 20, T, A, 17-OHP, and E2 had declined to levels slightly above normal. The baby had a 46,XX karyotype and was SRY negative (FiSH). Sonography revealed a normal uterus. Biochemically, the girl showed constantly elevated LH (59-67 $\mathrm{mU} / \mathrm{ml})$ and FSH $(112-142 \mathrm{mU} / \mathrm{ml})$ without measureable E2 $(<10 \mathrm{pg} / \mathrm{ml})$ on 3 occasions during the first 2 months of life. Urinary steroid profile showed normal excretion of CYP21 and CYP17 metabolites. Genetic testing for a CYP19A1 defect revealed a homozygous mutation in exon 5 (Glu210Lys). Conclusions: This is the 12th case of aromatase deficiency in a 46,XX individual reported so far. Interestingly, the mother's ovaries were enlarged with multiple cysts, which points at prolonged stimulation of LH receptors, leading to marked ovarian hyperandrogenism that persisted during the first weeks after birth. As a mechanism, we propose that lack of estrogen may have in- 
creased placental hCG and perpetuated ovarian steroid excess. Alternatively, increased fetal LHRH, driven by estrogen deficiency, may have crossed the placenta and stimulated maternal gonadotrophins. To our knowledge, the changes in the mother during the first weeks after birth have not been documented yet.

\section{EPO-250 Thyroid}

\section{Screening for hypothyroidism in special schools for children with learning difficulty in South Eastern Nigeria}

Maryann Ibekwe'; Roland Ibekwe ${ }^{2}$; Angela Okolos; Tim Cheetham ${ }^{4}$ ${ }^{1}$ Federal Teaching Hospital Abakaliki, Paediatrics, Abakaliki, Nigeria; 'University of Nigeria Teaching Hospital Enugu, Paediatrics, Enugu, Nigeria; ${ }^{3}$ University of Benin Teaching Hospital BninCity, Paediatrics, Benin City, Nigeria; ${ }^{4}$ Institute of Human Genetics Newcastle University, Paediatric Endocrinology, Newcastle-upon-Tyne, United Kingdom

Background: Congenial hypothyroidism $(\mathrm{CH})$ remains the leading cause of preventable mental impairment worldwide. Since there are as yet no screening programmes for congenital hypothyroidism in Nigeria, This study aimed to screen children attending special schools for the mentally impaired for undiagnosed hypothyroidism that may have been missed earlier.

Objective and hypotheses: To evaluate the prevalence of hypothyroidism in children with learning difficulty attending special schools.

Methods: Two schools for the mentally handicapped children located in Enugu and Abakaliki were used for the study. Using the dry blood test, blood samples were estimated for TSH and T4. Analysis was by time resolved fluoroimmunoassay using AutoDELFIA. Intellectual assessment- Intelligence quotient (IQ) was assessed using the Draw-A-Person test (DAPT). Height and weight Measurements were done using standard methods.

Results: A total of 690 pupils were seen out of which 117 (one hundred and seventeen) aged 3-18 years who met the criteria were selected in the final analysis. Sixty three $(53.8 \%)$ students were males while $54(46.2 \%)$ were females. The different health problems encountered among the subjects were categorised into autism $=8$, cerebral palsy $(C P)=13$, down syndrome $(D S)=16$, learning disability $(\mathrm{LD})=15$, mental retardation $(\mathrm{MR})=32$ and normal $=33$. The mean Height, weight, BMI SDs of the study group were $-0.6,0.2$ and 0.5 respectively. The mean TSH was $0.95 \mathrm{mIU} / \mathrm{L}$ with a range of $0.1-4.1 \mathrm{mIU} / \mathrm{L}$ and the mean T4 was $3.17 \mu \mathrm{g} / \mathrm{dl}$ with a range of $1.62-5.10 \mu \mathrm{g} / \mathrm{dl}$. There was no case with an elevated TSH above the cut off. Sixty seven point five percent $(67.5 \%)$ of the students had IQ less than 75 with a range of 0 to 71 while those with IQ above 75 were $32.5 \%$ with a range of $75-154$.

Conclusions: None of the subjects had elevated TSH. Congenital hypothyroidism may therefore not be a common cause of mental impairment in $\mathrm{Ni}$ geria.

\section{EP0-251 Thyroid}

\section{Thyrotoxicosis complicated with neutropenia} at first presentation in a paediatric patient Arundoss Gangadharan; Harsha Hanumanthaiah; Sze May Ng Southport and Ormskirk Hopsital NHS trust, Paediatrics, Ormskirk, United Kingdom

Introduction: Graves disease is a common cause of hyperthyroidism in paediatric age group. Lugol's iodine (LI) has been extensively used preoperatively in the management of thyrotoxicosis. We report a rare case of thyrotoxic Grave's disease complicated by neutropenia at first presentation in a 13 year old boy. We describe the successful use of Lugol's iodine as a primary treatment for treating the thyroid crisis, with no previous such case report in the paediatric literature.

Case report: A 13year old boy presented with a 3 months history of weight loss, insomnia, palpitations and anxiety. On examination, he had tachycardia, sweating, tremors, brisk deep tendon reflexes and a palpable smooth goitre. He had a prior 2year history of orthostatic proteinuria of unknown aetiology and was under the care of nephrologists. TSH was undetectable, free T4 was $32.9 \mathrm{pmol} / \mathrm{L}$, TRAB antibodies were positive, $\mathrm{Hb}$ was $11.5 \mathrm{~g} / \mathrm{dl}$, total white cell count $3.3 \times 109 / \mathrm{L}$ with a neutrophil count of $<1.5 \times 109 / \mathrm{L}$, and a platelet count of 137. ANA and ANCA antibodies were negative. There was no previous documented neutropenia. He was commenced on propranolol, and Lugol's iodine at $0.2 \mathrm{mls}$ TDS and he showed rapid clinical improvement with a normal neutrophil count after 16 days. He was then put on carbimazole and propranolol for 14 days and went on "Block and replace" (combined) therapy where antithyroid drugs prevent thyroid hormone production and thyroxine is then added in a replacement dose. He underwent total thyroidectomy after 6 months with no complications. He is now on long-term thyroxine replacement therapy.

Conclusion: In cases where thionamides are contraindicated due to the risk of side effects such as agranulocytosis, Lugol's iodine may be an alternative temporary form of first line therapy as it will block thyroid hormone synthesis and release, and can be administered in addition to a blockade.

\section{EPO-252 Thyroid \\ Comparative evaluation of therapy with L-thyroxine versus no treatment in children with idiopathic and mild subclinical hypothyroidism \\ Malgorzata Wasniewska'; Andrea Corrias ${ }^{2}$; Tommaso Aversa ${ }^{1}$ Mariella Valenzise'; Alessandro Mussa'; Lucia De Martino ${ }^{3}$; Fortunato Lombardo'; Filippo De Luca'; Mariacarolina Salerno ${ }^{3}$ 'University of Messina, Department of Pediatrics, Messina, Italy; ${ }^{2}$ University of Turin, Department of Pediatrics, Turin, Italy; ${ }^{3}$ University "Federico II" of Naples, Department of Pediatrics, Naples, Italy}

Objective: a) To evaluate auxological changes under L-T4 therapy and posttherapy evolution of thyroid function in 69 children with mild and "idiopathic" subclinical hypothyroidism SH (Group A); b) to compare our results with those recorded in $92 \mathrm{SH}$ untreated children (Group B).

Design: Group A children were treated for 24 months and their TSH and FT4 levels 3 months after therapy withdrawal (27 months) were compared with those measured in untreated Group B at the end of a 24-month follow-up.

Results: The prevalence of children who normalized TSH values at the end of follow-up was significantly higher in Group A, but the overall prevalence of the ones who either normalized or maintained unchanged TSH levels was very similar in the 2 groups, as well as that of children who exhibited a further TSH increase above $10 \mathrm{mU} / 1$. In Group A TSH values at 27 months were positively associated with the baseline ones. In both groups no significant clinical and auxological changes were observed during follow-up.

Conclusions: a) a two-year treatment with L-T4 in children with "idiopathic" and mild $\mathrm{SH}$ is not able to distinctly modify the natural course of $\mathrm{SH}$, at least in terms of growth and post-therapy outcome of hyperthyrotropinemia; b) therapy is not able to prevent the risk of a further increase of TSH after treatment withdrawal; c) post-therapy outcome of TSH serum levels is significantly conditioned by baseline TSH.

\section{EP0-253 Thyroid \\ Incidence of congenital hypothyroidism in pre- term neonates during the first month of life Mohammad Torkaman ${ }^{1}$; Fariba Ghasemi' ${ }^{1}$ :Mohammad Abyazi ${ }^{1}$. Amin Saburi \\ 1Baqiyatallah University of Medical Sciences, Pediatric department, Tehran, Islamic Republic of Iran; ${ }^{2}$ Baqiyatallah University of Medical Sciences, Chemical injury research center, Tehran, Islamic Republic of Iran}

Background: Congenital hypothyroidism (CHT) is one of the most important congenital endocrine disorders. The occurrence of CHT is estimated about 1 per 3000 newborns. The prevalence and etiology of CHT and abnormal thyroid screening tests is reported in different ranges.

Objective and hypothesis: It is possible that the prematurity of lung has relation with CHT. In this study we assessed the pre-term newborns for CHT and factors that influence thyroid function.

Methods: One hundred newborns under 35 weeks of gestational age were investigated. Baseline serum thyrotropin (TSH) and free thyroxine (FT4) levels were measured during the first 5 days of life, and repeated tests were performed during the first 5 weeks. We analyzed the effects of gestational age, gender, weight and the presence of respiratory distress syndrome on the alteration of thyroid function tests.

Results: The mean gestational age at delivery was $32.35 \pm 1.97$ (range, 28-35) weeks and CHT was observed in 13 preterm infants (13\%).(Table-1) Gestational age was the sole factor which affect the FT4 changes over the two 
weeks follow up ( $\mathrm{P}<0.001, \beta$ : -2.783 , Power: $70.2 \%)$ although the differences between baseline and follow up amount of TSH were not significantly associated with gestational age (P: 0.062, power: 46\%). Moreover, the adjusted TSH and FT4 serum level changes during follow up were significantly different between two groups (P: 0.006, 0.000, respectively).

Conclusion: It seems that thyroid function tests should be repeated in preterm infants, especially for patients with lower gestational age. Also, CHT should be considered among the newborns that are affected by RDS. Key word: Congenital Hypo-thyroidism; thyroid screening tests, pre-term neonate, respiratory distress syndrome. Group Male ratio Weight (less than $3 \mathrm{Kg}$ ) Gestational age (Weeks) RDS Surfactant CHT(N=13) 46.2\% 84.6\% 32.07 $33.1430 .8 \%$ $30.8 \%$ Normal $(\mathrm{N}=87) 44.8 \% 63.2 \% 33.12 \pm 2.0131 \% 33.3 \%$ P.value $>0.05$ 0.1310 .0020 .629 (adjusted 0.000$)>0.05$

\section{EPO-254 Thyroid \\ Gitelman syndrome and hyperthyroidism: is it related? \\ Nursel Muratoglu Sahin; Sibel Tulgar Kinik \\ Baskent University, pediatric endocrinology, Ankara, Turkey}

Background: Gitelman syndrome(GS) is an salt-losing renal tubulopathy that is characterized by hypomagnesemia, hypokalemia, hypocalciuria, and metabolic alkalosis.

Objective and hypotheses: Herein we reported a girl who was diagnosed with GS together with Graves disease(GD).

Case: A 16-year-old girl was admitted to our center with the complaints of tachycardia, palpitation and chest pain during the last 3 months. Her medical history revealed that she had complained excessive salt intake and hand spasms when she was 12 years old. During this period, hypokalemia, hypomagnesaemia and metabolic alkalosis were detected. As she had been diagnosed with GS, replacement treatments of potassium and magnesium were used. 2 months after this treatment, she started to complain about nervousness and hyperhydrosis. She was diagnosed with hyperthyroidism and propylthiouracil was started. Because of poor compliance, euthyroid status could not be achieved during those last 4 years. On physical examination, we found that her skin was sweaty and warm, she was tachycardic, and had goiter. Laboratory tests revealed severe hypokalemia, hypomagnesaemia suppressed TSH levels with high free T4 and free T3 levels and the presence of anti-TSH receptor antibodies, which led to the diagnosis of GD. She was treated with propranolol, replacement of potassium and magnesium, dexamethasone and propylthiouracil $(300 \mathrm{mg} /$ day). As hyperthyroidic state sustained, carnitine, cholestyramine was added to her treatment and then lugol's solution was given before thyroidectomy. Near total thyroidectomy was performed when she was euthyroid. L-thyroxine treatment was started in postoperative second week. Severe cardiomyopathy and systolic dysfunction persisted although she was euthyroid and she died 3 months after surgery.

Conclusions: In literature, only one patient has been reported with a cooccurrence of GS and GD. Which mechanisms act in this occurrence is not known yet. We thought that, GD accompanying to GS is the main cause of the mortal outcome of the patient.

\section{EPO-255 Thyroid \\ Congenital anomalies in infants with congenital hypothyroidism in Qazvin, Iran \\ Fatemeh Saffari; Yeva Melikian \\ Qazvin University of medical sciences, Pediatrics, Qazvin, Islamic \\ Republic of Iran}

Background: In the last decade a high frequency of extrathyroidal congenital anomalies has been reported in infants with congenital hypothyroidism detected by neonatal screening. Aproximately $10 \%$ of infants with congenital hypothyroidism have associated congenital anomalies. Cardiac anomalies are most common, but anomalies of the nervous system and eye have also been reported.

Objective: The main objective of this study was identification of associated disorders in patients with congenital hypothyroidism.

Methods: This descriptive study was performed on newborns with congenital hypothyroidism that had been diagnosed by screening in Qazvin province of Iran from 1385 to 1390 . The SPSS software was used for analysis of data and results was reported in the form of tables and graphs. Variables were analyzed by $\mathrm{Chi}-$ square test.
Results: In the screening 287 newborns with congenital hypothyroidism were diagnosed -122 female (42.2\%). $36(12.5 \%)$ of 287 patients had congenital anomalies. 10 (3.5\%) Down syndrome, 10 (3.5\%) cardiaovascular anomalies , $9(3.1 \%)$ hearing disorder, $2(0.7 \%)$ eye disorder, $2(0.7 \%)$ metabolic disease, $2(0.7 \%)$ disorder of face and ear, $2(0.7 \%)$ CNS disorder, one $(0.3 \%) \mathrm{CAH}$, one $(0.3 \%)$ panhypopituitrism and one $(0.3 \%)$ pseudohypoparathyroidism were diagnosed.

Conclusion: With respect to the association of congenital anomalies with congenital hypothyroidism, every newborn with congenital hypothyroidism should be carefully examined for cardiaovascular disorders and other related anomalies.

Key words: Congenital hypothyroidism, additional congenital anomalies, infants

\section{EPO-256 Thyroid \\ Reversible growth failure and complete GH deficiency in a 4-year-old girl with misdiagnosis of hypothyroidism}

Laura Gaspari; Francoise Paris; Charles Sultan

Service de Pédiatrie 1, Hôpital Arnaud-de-Villeneuve, CHU Montpellier et Université Montpellier 1, Unité d'Endocrinologie-Gynécologie

Pédiatrique, Montpellier, France

Background: Chronic lymphocytic thyroiditis (CLT) is a common cause of hypothyroidism in adolescence, but very rare in infancy. Neonates born to mothers with thyroid dysfunction need additional thyroid function monitoring, since thyroid autoimmune disease, often uncontrolled during pregnancy, is a risk factor of primary thyroid dysfunction in early infants. We report a case of reversible growth failure and complete GH deficiency (GHD) in a 4-year-old girl with misdiagnosis of hypothyroidism.

Case report: A 4-year-old girl presented with severe short stature (height $<$ $3 \mathrm{SD}$ ), as she had stopped growing at 2 years old. The parents reported fatigue and constipation. Physical examination revealed dry and gray-sallow skin, bloated abdomen, but no goiter. Hormonal evaluation revealed dramatically low IGF-1 $(<25.0 \mathrm{ng} / \mathrm{ml})$ and IGF-BP3, along with complete GHD $(<3 \mathrm{mU} / \mathrm{L}$ for 2 stimulation tests). TSH was $629.5 \mathrm{mIU} / \mathrm{ml}$, free T4 was $0.08 \mathrm{ng} / \mathrm{dl}$, and prolactin level was $17.2 \mathrm{ng} / \mathrm{ml}$. Bone age was 2 years. Antibodies to thyroglobulin and thyroid peroxidase were strongly positive, suggesting CLT. CNS MRI showed anterior pituitary hyperplasia. After 6 months of therapy with thyroid hormones, she presented no signs or symptoms suggestive of hypothyroidism and accelerated growth velocity $(1 \mathrm{~cm} / \mathrm{month})$; hormonal evaluation revealed TSH of $2.4 \mathrm{mIU} / \mathrm{ml}$, free T4 of $1.71 \mathrm{ng} / \mathrm{dl}$, and prolactin level of $3.2 \mathrm{ng} / \mathrm{ml}$. IGF-1 and IGF-BP3 were respectively 150.0 and $3890 \mathrm{ng} / \mathrm{ml}$. MRI imaging showed a regression of pituitary hyperplasia. Medical records established that the patient's mother had received uncontrolled levothyroxine therapy for Hashimoto's thyroiditis throughout pregnancy and that the Guthrie screening test in the 1 st week of life was normal.

Conclusions: Despite clinical and hormonal data suggestive of primary GHD, we should consider hypothyroidism as a possible and treatable cause of growth failure also in early infancy, in relation to the reversible pituitary somatotropic cell compression by thyreotropic cell hyperplasia.

\section{EPO-257 Thyroid \\ The natural history of hyperthyrotropinemia in the first year of life in healthy newborns Joseph Meyerovitch'; Nofar Amitai'; Irit Schushan-Eisen ${ }^{3}$ \\ 'Schneider Children's Medical Center of Israel, Institute for Endocrinology and Diabetes, National Center for Childhood, Petah Tikva, Israel; ${ }^{2}$ Tel-Aviv University, Sackler School of Medicine, Tel Aviv, Israel; ${ }^{3}$ The Edmond and Lili Safra Children's Hospital, Sheba Medical Center, Department of Neonatology, Ramt Gan, Israel}

Background: Thyroid deficiency in infancy can cause irreversible mental retardation and developmental disorders. Neonatal screening tests are available for early detection of congenital hypothyroidism. However, few studies have investigated the natural history of hyperthyrotropinemia during the first year of life in healthy newborns.

Objective and hypotheses: To investigate the natural history of initial abnormal thyrotropin stimulating hormone (TSH) levels in healthy newborns, and to assess the risk of requiring Eltroxin treatment.

Patients and methods: The study group included all healthy newborns 
$(\mathrm{n}=18,507)$ born at Sheba Medical Center, a tertiary university-affiliated hospital, in 2002-2007 and insured by Clalit Health Services who underwent measurements of TSH and free T4 (FT4) in the first year of their life and thereafter until 2010. The patients were identified by database review and the clinical and laboratory data were collected from the medical files.

Results: A total of $652(3.5 \%)$ infants met the study criteria. The initial workup included thyroid ultrasound $(\mathrm{n}=32,47.7 \%)$, thyroid isotope scanning $(\mathrm{n}=2,3.0 \%)$, and thyroid antibodies $(\mathrm{n}=9,13 \%)$. TSH was within normal limits in $89.7 \%(\mathrm{n}=585)$ of infants, above normal range but less than $10 \mathrm{mIU} / \mathrm{L}$ (minimally raised) in $9.5 \%(\mathrm{n}=63)$, more than $10 \mathrm{mIU} / \mathrm{L}$ in $0.6 \%(\mathrm{n}=4)$, and suppressed in $0 \%$. Six infants $(0.9 \%$ of the cohort, $8.9 \%$ of the subgroup with high TSH levels) were treated with Eltroxin, which was initiated at TSH levels of 5.8-10.9 mIU/L.

Conclusions: TSH level is measured infrequently during the first year of life in normal healthy infants. Most measurements are within normal limits. Only a minority of normal newborns escape the thyroid neonatal screening program. Nevertheless, $8.9 \% \%$ of the infants investigated in the present study received thyroid hormone supplement for a period of time. Clinicians should take these findings into consideration when deciding to initiate Eltroxin treatment in an otherwise healthy newborn with hyperthyrotropinemia.

\section{EPO-258 Thyroid}

Goiterous congenital hypothyroidism and a new heterozygous mutation in the THOX2 gene; epiphenomenon or clinical relevance?

Joachim Pohlenz'; Annette Richter-Unruh'; ${ }^{2}$ Andreas Schuster ${ }^{2}$

JJohannes Gutenberg University, Rheinland Pfalz, Mainz, Germany;

${ }^{2}$ Endokrinologikum Ruhr, NRW, Bochum, Germany

Background: In congenital primary hypothyroidism (CH) $20 \%$ of cases are due to defects in thyroid hormone genesis. In some cases with iodide organification defects the hypothyroidism was only mild and transient especially if there is a heterozygous mutation in the thyroid oxydase 2 gene (THOX2). Objective and hypotheses: To differentiate a transient to permanent hypothyroidism we followed up a patient with a novel heterozygous mutation in the THOX2 gene.

Methods: One patient with mild elevation of TSH in the neonatal screening programme was suspected of dyshormonogensis, because of mild goitre and a suspicious ultrasound scan. We studied the coded exons of TPO-, PDS-, NIS-, TG-, THOX2- and THOX2A genes.

Results: We found a novel heterozygous mutation in the THOX2 gene in the exon 17: c.2182 G>A; p.A728T. In the mother we identified the same heterozygous mutation. Father and sister showed wild type sequences. Therefore we discontinued the replacement therapy at the age of 2 years and 6 month for 4 weeks. The TSH was rising up to $80,0 \mu \mathrm{IU} / \mathrm{ml}$ and the fT4 went down to $7,6 \mathrm{ng} / \mathrm{ml}$. The thyreoglobin level was in a normal range. In the ultrasound scan the thyroid volume was enlarged and showed a diffuse hyper vascularisation. The replacement therapy was started again.

Conclusions: In the literature heterozygous THOX2 gene mutations are associated with a mild transient hypothyroidism.

In our patient we found a goiterous neonatal hypothyroidism with a heterozygous mutation in the THOX 2 gene, which is not described yet. In discontinuing the therapy we could show, that a permanent hypothyroidism is probably. Because of the mother is carrying the same mutation, a association to the goitre in the patient is doubtfully.

\section{EPO-259 Thyroid}

A two day-old hyperthyroid neonate with resistance to thyroid hormone born to a mother with well-controlled Graves' disease

Shuichi Yatsuga'; Y Yuji Hiromatsu; Shigekazu Sasaki;

Hirotoshi Nakamura ${ }^{3}$ K Koju Katayama'; Junko Nishioka';

Yasutoshi Koga ${ }^{1}$

${ }^{1}$ Kurume University School of Medicine, Department of Pediatrics and Child Health, Kurume, Japan; ${ }^{2}$ Kurume University School of

Medicine, Division of Endocrinology and Metabolism, Department of Medicine, Kurume, Japan; ${ }^{3}$ Hamamatsu University School of Medicine, Department of Internal Medicine, Hamamatsu, Japan

Background: Resistance to thyroid hormone (RTH) is a syndrome caused by thyroid hormone receptor $\beta$ (TR $\beta$ ) mutations, which are usually inherited in an autosomal dominant pattern. The patient had an R243W mutation in TR $\beta$. The mother did not carry this mutation; however, the R $243 \mathrm{~W}$ TR $\beta$ mutation was present in the non-symptomatic father.

Case presentation: The patient happened hyperthyroid symptoms at 2 dayold Japanese origin female neonate. Although the patient was diagnosed with $\mathrm{RTH}$, the hyperthyroid symptoms continued for 2 weeks. Therefore, the patient was treated methimazole (MMI) and iodine for 2 weeks without side effects, and has been no symptoms since treatment.

Conclusions: This is the first case report of a RTH neonate presenting with hyperthyroid symptoms born to a mother with Graves' disease, and treated MMI and iodine. MMI and iodine for short term may be optional treatment in symptomatic RTH neonates.

\section{EPO-260 Thyroid \\ lodine deficiency and of its control in central Siberia \\ Irina Osokina}

State Research Institute for Medical Studies of the North,

Endocrinology, Krasnoyarsk, Russian Federation

Background: The problem of iodine deficiency in Russia is very actual. In spite of the well-known methods of iodine prophylaxis the mechanisms of their effective application haven't been found in Russia so far. Siberia traditionally belonged to iodine-deficient regions. Our investigations (1997-2004) show that in the Central Siberia there is a serious natural iodine deficiency influencing the health of the population and demanding continuous adequate iodine prevention.

Objective and hypotheses: The aim of the research was to estimate the effectiveness of IDD prevention in Central Siberia according to the data of the neonatal TSH-screening.

Methods: Screening for congenital hypothyroidism used as an indicator of the degree of iodine deficiency and of its control (F. Delange, 1998). We analyzed the TSH values of 35000 infants born in 2009 (21000 - in Krasnoyarsk region, 6000 - in the Republic of Khakasia and 8000 -in the Republic of Tyva). Results: According to the congenital hypothyroidism screening the rate of TSH more than $5 \mathrm{mU} / 1$ was $11.8 \%$ in the Krasnoyarsk region (23.9\% in 2000) and corresponded to mild iodine deficiency. Transient hypothyroidism was revealed in $0.36 \%$ of the neonates $(0.5 \%$ in 2000$)$. In different regions of the Krasnoyarsk territory the TSH rate more than $5 \mathrm{mU} / 1$ in the newborn varied from $3.5 \%$ to $23.7 \%$. The values more than $20 \%$ were marked in the North (in the Evenkia and in the Taimyr). In the Republic of Khakasia the rate of TSH levels more than $5 \mathrm{mUn} / 1$ was $12.2 \%$ (9.5\% in 2000) it corresponding to mild iodine deficiency. Transient hypothyroidism was revealed in $0.11 \%$ of the neonates $(0.3 \%$ in 2000). In the Republic of Tyva located in the South of Siberia - TSH levels more than $5 \mathrm{mUn} / 1$ was $6.0 \%$ (38.6\% in $1997 ; 11.5 \%$ in 2000) it corresponding to mild iodine deficiency. Transient hypothyroidism was revealed in $0.1 \%(8.5 \%$ in $1997 ; 0.8 \%$ in 2000$)$.

Conclusions: Our investigations show that a serious iodine deficiency eliminated in Siberia, but there is a mild iodine deficiency demanding continuous adequate iodine prevention. 


\section{EPO-261 Thyroid}

A rare cause of failure to wean from mechanical ventilator support: hypothyroidism Ibrahim Cansaran Tanidir'; Tolga Unuvar ${ }^{2}$; Hasan OnaR; Aliye Sevil Sarikaya ${ }^{2}$; Erdal AdaR

${ }^{1}$ Mehmet Akif Ersoy Thoracic and Cardiovascular Surgery Training and Research Hospital, Thoracic and Cardiovascular Surgery, Istanbul, Turkey; ${ }^{2}$ Republic of Turkey Ministry of Health Kanuni Sultan Süleyman Training and Research Hospital, Department of Pediatric Endocrinology, Istanbul, Turkey

Background: Duration of intensive care after complex pediatric cardiac surgery may be longer than expected. However, longer intensive care stay may lead to secondary diseases, some of which can affect the recovery time.

Case report: A systemic-pulmonary artery shunt (Mee Shunt) operation was performed on a 5 month-old-girl with pulmonary atresia and ventricular septal defect. After surgery, she could not wean from mechanical ventilator support despite several attempts. The longest duration of extubation was 72 hours. She failed to wean from mechanical ventilator support after second surgery as well. Elevated TSH [79.2 mIU/mL (normal, 0.4-5.0 mIU/mL)] and free T4 levels [0.6 ng/dL (normal, 0.9-2.6 ng/dL)] were found although they were normal preoperatively. Thyroid ultrasound was normal. Thyroid dysfunction was considered to be related to iodine exposure or dyshormonogenesis. The patient was treated with levothyroxine $50 \mathrm{mcg}$ /day and could be extubated on the fourth day and discharged on the tenth day. The patient is currently ninemonth-old and under levothyroxine treatment ( $3 \mathrm{mcg} / \mathrm{kg} / \mathrm{day})$.

Conclusion: Although development of clinically significant hypothyroidism is rare during intensive care stay, thyroid dysfunction should be considered in all patients who fail to wean from mechanical ventilation after surgery for congenital heart diseases.

\section{EPO-262 Thyroid \\ Thyroid dysfunction in a child treated with amiodarone \\ Karine Braun; Aurélie Armougon; Amel Mathiron; Hélène Bony}

$\mathrm{CHU}$ Amiens, Pediatrics, Amiens, France

Background: Amiodarone, molecule rich in iodine is frequently used in arrhythmia of the child. Amiodarone may be responsible for thyroid dysfunction. Toxicity is expressed at different points of impact of thyroid regulation: pituitary, thyroid, target tissues.

Objective and hypotheses: The aim of this study is to determine the proportion of patients receiving amiodarone who develop thyroid dysfunction and to type this thyroid dysfunction.

Methods: This retrospective study, which took place between January 2006 and December 2010, included any patient less than 18 years receiving amiodarone for rhythm disorder. Patients excluded: prenatal rhythm disorder, thyroid dysfunction known before treatment with amiodarone. The cases were clinically investigated prior to, during and after treatment by thyroid sonography, thyroid function test (TSH, FT3, FT4) and antithyroid antibodies.

Results: 43 patients were followed, out of 49 patients included. Median age of initiation of treatment is 2.07 months [ 1 day - 181 months]. Mean treatment duration is 28.04 months [ $1-200$ months] Overall rate of thyroid dysfunction is $23 \%$. We describe 3 types of thyroid dysfunction.

$\begin{array}{lccc}\text { Number of } & \text { Hyperthyroidism } & \text { Hypothyroidism } & \text { Compensed hypothyroidism } \\ \text { patients } & 2 & 3 & 5 \\ \begin{array}{l}\text { Median time } \\ \text { to onset }\end{array} & \begin{array}{c}7 \text { years and } 8 \text { months } \\ ( \pm 5 \text { years and } 9 \text { months })\end{array} & \begin{array}{c}26 \text { days } \\ ( \pm 38 \text { days })\end{array} & \begin{array}{c}2 \text { years and } 8 \text { months } \\ ( \pm 2 \text { years and } 6 \text { months })\end{array} \\ \begin{array}{c}\text { Theatment } \\ \text { stop amiodarone, } \\ \text { Carbimazole } 1 / 2, \\ \text { Corticoidsteroids } 2 / 2\end{array} & \begin{array}{c}\text { stop amiodarone, } \\ \text { L-thyroxine } 3 / 3\end{array} & \begin{array}{c}\text { stop amiodarone, } \\ \text { L-thyroxine } 3 / 5\end{array}\end{array}$

Two periods at risk were identified: an early phase, in the first 3 months of treatment, with a hypothyroidism risk and a later phase with a hypothyroidism or thyrotoxicosis risk.

Conclusions: The occurrence of thyroid dysfunction is not unimportant in children. A careful monitoring of thyroid complications is necessary with a complete blood test before treatment, monitoring at 3 months and every six months, until one year after stopping treatment. Limit the duration of treatment is mandatory in order to prevent the iodine-induced thyrotoxicosis.

\section{EPO-263 Thyroid}

Aspergillus thyroiditis as a clinical diagnosis in a patients with disseminated aspergillus infection

Jimmy Nguyen ${ }^{\text {; }}$ Carla Minutti; ; Ricarchito Manera ${ }^{3}$

${ }^{1}$ Loyola University Medical Center, Pediatrics, Chicago, United States; ${ }^{2}$ Loyola University Medical Center, Pediatric Endocrinology, Chicago, United States; ${ }^{3}$ Loyola University Medical Center, Pediatric Hematology Oncology, Chicago, United States

Background: Aspergillus thyroiditis is an uncommon condition seen in the setting of disseminated aspergillus disease affecting immunocompromised individuals. Diagnosis is usually made by fine needle aspiration or biopsy. Objective and hypotheses: In patients already diagnosed with aspergillus infection, aspergillus thyroiditis may be a clinical diagnosis and invasive confirmative studies may not be needed if management would not be affected.

Method: We present a case report and review available literature.

Results: We present a case of a male patient with clinically diagnosed aspergillus thyroiditis who did well with voriconazole therapy and frequent monitoring of thyroid status. Review of the literature suggest that definitive diagnosis of aspergillus thyroiditis may not be needed as management decisions are not affected.

Conclusions: Definitive diagnosis of aspergillus thyroiditis by FNA or biopsy in the setting of immunocompromised patients already diagnosed with disseminated aspergillus may not contribute to management.

\section{EPO-264 Thyroid \\ A case of childhood cancer survivor (CCS) with Graves' disease \\ Eiichiro Satake; Yuichi Nakagawa; Toshiki Nananishi; \\ Rie Matsushita; Yasuko Fujisawa; Shinichiro Sano; \\ Shinichi Nakashima; Eiko Nagata; Kimiyoshi Sakaguchi; \\ Shuichi Okada; Tsutomu Ogata \\ Hamamatsu University School of Medicine, Pediatrics, Hamamatsu, \\ Japan}

Background: Thyroid disease is common in patients with childhood cancer survivors (CCS) who have been treated with radiation or bone marrow transplantation (BMT). Most of them are hypothyroidism and the case reports of CCS with Graves' disease are limited.

Case report: A 12-year old female came to our hospital for following up. She was diagnosed acute lymphoblastic leukemia (ALL) at 4 years old, and treated chemotherapy. At 11 years old, she relapsed ALL and was treated chemotherapy again and was performed allogeneic BMT with total body irradiation (TBI) at a dose of 12 Gy. One year after BMT, she exhibited low TSH $(<0.01 \mu \mathrm{U} / \mathrm{ml})$ without any manifestations related to hyperthyroidism (body weight change or tachycardia). She has no family history of thyroid disease. Although we decided simple observation, she felt fatigue and tachycardia (HR 109/ $\mathrm{min}$ ) after 3 months. Laboratory data were, TSH $<0.01 \mu \mathrm{U} / \mathrm{ml}$, fT4 $2.2 \mathrm{ng} / \mathrm{dl}, \mathrm{fT} 37.2 \mathrm{pg} / \mathrm{ml}$ and TRAb $9.9 \mathrm{IU} / \mathrm{l}$. In ultrasonography, her thyroid was slightly enlarged and thyroid blood flow was generally increased. Technetium 99m (99mTc) pertechnetate scanning exhibited high uptake $(5.6 \%)$. We diagnosed Graves' disease, and started MMI (15 mg/ day) for treatment. Her manifestation rapidly disappeared and thyroid hormones were normalized after 3 weeks. Her clinical manifestations were mild and she showed good response to MMI therapy.

Conclusions: This is a rare case of CCS with Graves' disease after BMT. We suppose that manifestation of the hyperthyroidism of CCS is mild because of the effect of irradiation to the thyroid. 
EPO-265 Thyroid

Postnatal regression of thyroid gland in the cases with congenital hypothyroidism

Alev Oguz Kutlu

Dr. Sami Ulus Children's Hospital, Pediatric Endocrinology, Ankara, Turkey

Background: Patients with congenital hypothyroidism $(\mathrm{CH})$ were re-evaluated with thyroid scintigraphy (TS) and thyroid ultrasonography (TU) in recent years. In some studies, thyroid gland couldn't be detected in cases with thyroid hypoplasia and authors suggested that the initial diagnosis was wrongly set up as thyroid hypoplasia.

Objective and hypotheses: We are presenting cases with congenital hypothyroidism in which thyroid gland couldn't be visualised in ultrasonographic examination of neck with different indications. After this incidental finding, we measured serum levels of thyroglobulin and calcitonin, and TS (only for one case) was applied to show the possible apoptosis of the gland in years. Methods: Four cases with congenital hypothyroidism due to thyroid hypoplasia (3) and dyshormonogenesis (1) are evaluated. Ultrasonographic examination applied for different reasons while they were $2.5,12,8$ and 10 years old, respectively.

Results:

\begin{tabular}{|c|c|c|c|c|c|c|c|}
\hline & Initial TFT & $\begin{array}{l}\text { Thyroid } \\
\text { volume in } \\
\text { first TU (ml) }\end{array}$ & First TS & Etiology & $\begin{array}{c}\text { Control } \\
\text { TS }\end{array}$ & $\begin{array}{c}\mathrm{Tgb} \\
\text { (ng/ml) }\end{array}$ & $\begin{array}{c}\text { Calcitonin } \\
(\mathrm{pg} / \mathrm{ml})\end{array}$ \\
\hline & $\begin{array}{l}\text { FT4: } 0.38 \mathrm{ng} / \mathrm{dL} \\
\text { TSH: } 100 \mathrm{IU} / \mathrm{mL}\end{array}$ & 0.84 & Gland + & Hypoplasia & $\begin{array}{c}\text { No } \\
\text { gland }\end{array}$ & 1.43 & 2.35 \\
\hline & $\begin{array}{l}\text { FT4: } 0.98 \mathrm{ng} / \mathrm{dL} \\
\text { TSH: } 2.1 \mathrm{IU} / \mathrm{mL}\end{array}$ & 1.02 & Gland + & Hypoplasia & - & 2.50 & 0.50 \\
\hline & $\begin{array}{l}\text { FT4: } 0.98 \mathrm{ng} / \mathrm{dL} \\
\mathrm{TSH}>100 \mathrm{IU} / \mathrm{mL}\end{array}$ & 3.10 & Gland + & $\begin{array}{l}\text { Dyshormo- } \\
\text { nogenesis }\end{array}$ & - & - & - \\
\hline 4 & $\begin{array}{l}\text { FT4: } 0.68 \mathrm{ng} / \mathrm{dL} \\
\text { TSH>100 } \mathrm{IU} / \mathrm{mL}\end{array}$ & 1.80 & Gland + & Hypoplasia & 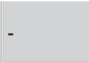 & 3.18 & 1.89 \\
\hline
\end{tabular}

Conclusions: We are suggesting that the thyroid gland could be regressed in years as the gland visualised in TS during initial evaluation. Regression can be related with inhibition of thyroid growth factors or shortened survival of thyrocytes. Pathological atrophy of endocrine-dependent organs after pathological withdrawal of trophic hormonal stimulation is accompanied by a massive wave of apoptosis.

It was shown that TSH, a thyroid growth factor, inhibited apoptosis and inhibition of TSH induced apoptosis in animal studies. Insufficient stimulation or apoptosis of gland can be explained with suppression of TSH to lower limits as a consequence of treatment. This is the first report suggesting apoptosis of thyrocytes in patients with $\mathrm{CH}$

\section{EPO-266 Thyroid}

\section{A cohort of thyroid cancer in children and adolescents}

Catherine Piquard; Danielle Rodrigue; Pierre Bougneres

Hospital Bicetre, Pediatric Endocrinology, Le Kremlin Bicetre, France

Background: Thyroid nodules in childhood and adolescence are less prevalent but more often malignant than in adulthood. Malignant nodules are predominantly papillary thyroid cancers.

Objective: To determine if a fine needle aspiration of thyroid nodules (FNA) is contributive in management in children and adolescents with thyroid nodules.

Population and methods: 7 children (1 girl, 6 boys, mean age 11,5 yr, range 5-15,4 yr) between 1997 and 2012 followed in the service, presented thyroid nodules (mean diameter $30,7 \mathrm{~mm}$, range 13-53 mm). Positive personal history of thyroid disease was found in $71 \%$ of the cases: $3 / 7$ Hashimoto thyroiditis and 2/7 congenital dyshormonogenetic hypothyroidism. At diagnosis, 6/7 had a goiter and 6/7 a palpable nodule. Ultrasonography, FNA, scintigraphy and thyrocalcitonin dosage were performed before surgery on all patients.

Results: FNA was contributive in $57 \%(4 / 7)$ of these cases in which ultrasound showed microcalcifications in 3 out of $4.3 / 7$ patients with normal FNA had papillary thyroid cancer. All were papillary thyroid cancer with positive lymph nodes in 5 cases, associated with adrenal metastasis for one patient.

Conclusions: $71 \%$ of papillary thyroid cancers were metastasized at diagnosis in this cohort. Clinical follow up of any child having a thyroid disease must include an ultrasound monitoring once a year to diagnose nodules. Given the high incidence of cancer, often with delayed diagnosis, thus metastases, and the limited reliability of exploratory investigations in children, our management of thyroid nodules in children is now almost systematically surgical. Any nodule should be removed and analyzed and the time management must be fast.

\section{EP0-267 Thyroid}

\section{Thyroid function tests and ultrasound comparative results in obese and DMT1 children}

Petrovic Rada SP'; Silvija Sajic ; Lesovic Snezana ${ }^{3}$

${ }^{1}$ Medical Center Cacak, Pedijatric Endocrinology, Cacak, Serbia;

${ }^{2}$ University Child Clinic Belgrade, Pedijatric Endocrinology, Beograd,

Serbia; ${ }^{3}$ Medical Institution Cigota, Pedijatric Endocrinology, Zlatibor, Serbia

Background: Thyroid function testa and Ultra sound (US) examination is necessary method in evaluation thyroid gland

Objective: To investigate relation of thyroid disfunction, (detected hormonal disturbancy) and results screened by thyroid ultrasound (US), in children with Obesity (Met Sy) and Diabetes Mellitus T1.

Methodology: US was performed in 452 pedijatric patients, dyring three years (from 2009-2011), obtained thyroid dimenzion( istmus, UPD,UPS) and morfolology (cystic area, izoehogenic and heteroehogenic nodus). Functional tests evaluated hormons (TSH,fT3,fT4), autoimmunity (At TPO, Tg, on TSH $\mathrm{r}$, Kalcitonon), find dysfunctional thyroid- subclinical and clinical hypo and hyper function. Blood samples were drawn from an antecubital wein and centrifuged after coagulation electro-hemiluminescence-immunoassay method Obesity evaluated according BMI and Met Sy criteria. Children with DMT1 are on insulin therapy regimen more than one year.

Results: Sample of $(298 \mathrm{f}, 154 \mathrm{~m})$ pediatric patients, evaluated according sex and age (from 9 to 17, -med 15,3), actually desease (obese 186 /Met Sy 46, DMT1 44) . Data find US changes on $232(51 \%)$ children, $67 \%$ patients $(43 \%$ ower standard dimension and $14 \%$ morphology), more in obese children Registered 3,3\% with clinical hypofunction,more in obese, with out sex difference. Subclinical hyper function 3\%(detected among f from 12-14 ages); Hypo or hyper function detected equal among $\mathrm{m}$ and $\mathrm{f}$ from 14-16 ages. Subclinical hypofunction with poz autoimunity with morphology changes persist $11 \%$ in DMT1, more than obese, predominant in $\mathrm{f}$. Hashimotto $16 \%$ in DMT1. US screen at 3 children indicated surgical therapy. From 20 patients, 4\%medicament threeted, (14 L-tiroxin, 3 PTU), 0,7\% had surgical operation ( 3 children, one girl of 12 years with Ca papillare)

Conclusions: Thyroid dimension correlate with age and BMI. Morfology changes registred more in obese than in DMT1 Autoimunity and associated disfunction in DMT1 predominant in girls in younger ages Obese children are at the same sex risk for thyroid disfunction.

\section{EPO-268 Thyroid \\ Congenital hypothyreosis in low income countries}

Vjosa Mulliqi Kotori'; Afrim Kotori'; Melehate Zhuri

${ }^{1}$ Pediatric Clinic University Clinical Center, Endocrinology, Prishtine, Kosovo; 'Laboratory Diagnostics Li-ori, Clinical Biochemistry, Prishtina, Kosovo

Introduction: One of the most common preventable cause of mental retardation is Congenital hypothyroidism. Since the clinical manifestations are often subtle or not present at birth, screening the primary thyroid stimulating hormone has became a standard in many parts of the world but still it remains missing in third world countries as is Kosovo. Despite the critical importance of TH on multiple organ systems, especially the brain, most infants with $\mathrm{CH}$ appear normal at birth. This protection is part of placental transfer of material $\mathrm{TH}$ and increased intracerebral conversion of $\mathrm{T} 4$ to $\mathrm{T} 3$ resulting in increased availability of $\mathrm{T} 3$ despite the low concentrations.

Case report: 6 months old boy, weight $4.5 \mathrm{~kg}$. with clinical signs of poor feeding, large fontanels, macroglossia, hypotonic and distended abdomen with umbilical hernia was tested for the first time at that age for hypothyreosis. Laboratory testing of TSH was $650 \mathrm{mIU} / 1, \mathrm{~T} 4<4 \mathrm{mcg} / \mathrm{dl}$, scintigraphy with iodine (123) identifies agenesis of thyroid gland. 12 months old giirl, weight $11 \mathrm{~kg}$, with horse cry, mild hypotonia, was tested for the first time for hypothyreosis at that age. TSH was $350 \mathrm{mIU} / 1, \mathrm{~T} 4<4 \mathrm{mcg} / \mathrm{dl}$, scintigraphy 
with iodine identify ectopic and hypoplastic thyroid gland.

Conclusion: Overlooked and delayed diagnosis leads to the most severe outcome of $\mathrm{CH}$, mental retardation, emphasizing the importance of neonatal screening. The cost of screening for $\mathrm{CH}$ is much lower that the cost of diagnosing $\mathrm{CH}$ at an older age. We should put an effort on screening programs for $\mathrm{CH}$ in Kosovo and other parts of the world when is still missing.

\section{EPO-269 Turner Syndrome \\ A very rare karyotype in TS syndrome: 45XO, 46X, I (Xq10), X, i(Xq10), i(Xq10)}

Maria Francesca Messina; Rosi Civa; Giuseppina Salzano;

Antonino Randazzo; Filippo De Luca

University of Messina, Department of Pediatrics, Messina, Italy

Background: Turner syndrome affects about one in 2500 liveborn females. It results from the haplo-insufficiency of some or all genes on the X-chromosome due to the complete absence of one X-chromosome or from structural anomalies of one X-chromosome. The phenotype is variable and depends on the chromosomal pattern. The condition is characterized, in its classical form, by short stature and gonadal failure, the latter resulting in delayed or absent pubertal development. Other common elements include lymphedema, renal and cardiac anomalies, webbed neck, cubitus valgus and are mainly present in patients with X-monosomy. Most TS patients have a mild phenotype; so the diagnosis is often delayed because of lack of suspicion. This is frequent for those patients who have rare karyotypic constitutions.

Case report: We present the case of a girl who was referred to our Unit of Pediatric Endocrinology for obesity. He was 16 years old and had not presented menarche. On physical examination she had: height $152.0 \mathrm{~cm}$ (- 1.7 SDS), mid-parental height $158.2 \mathrm{~cm}(-0.9$ SDS $)$, weight $83.400 \mathrm{~kg}(+2.5 \mathrm{SDS}), \mathrm{BMI}$ 36 , sitting height $/$ height $=0.545$, Tanner stage B2, P5, thyroid palpable, ungueal dysplasia, numerous cutaneous nevi, mild facial dysmorphic features. Laboratory investigation showed elevated levels of FSH $(79 \mathrm{mIU} / \mathrm{ml})$ and $\mathrm{LH}$ $(13 \mathrm{mIU} / \mathrm{ml})$ and pelvic ultrasound evidenced small ovaries $(1 \mathrm{cc})$ without follicles, so karyotype analysis was performed and confirmed the clinical suspicion of TS but evidenced a very rare chromosomal variant : $85 \%$ of the total cells with $45 \mathrm{XO}, 11 \%$ with $46 \mathrm{X}$, I (Xq10), 4\% with 47, X, i(Xq10), i(Xq10). Conclusions: Our case report confirms the theory that many, if not most, mosaic individuals with TS remain undiagnosed during infancy because they have a relatively unremarkable phenotype and that the severity of the phenotype classically associated with TS is exaggerated.

\section{EPO-270 Turner Syndrome \\ The respose of the GH treatment in patients with Turner syndrome \\ Se Young Kim ${ }^{1}$; Eun Young Kim²}

'Bundang Jesaeng General Hospital, DMC, Pediatric Endocrinology,

Pediatrics, Seongnam, Republic of Korea; ${ }^{2}$ School of Medicine,

Chosun University, Pediatrics, Gwangju, Republic of Korea

Background: Review of medical records from 27 patients with Turner syndrome treated with recombinant human growth hormone for more than 3 years was done. Differences in the changes in standard deviation scores for height were measured and grouped according to the karyotype and age at the start of treatment.

Methods: Review of medical records from 27 patients with Turner syndrome treated with recombinant human growth hormone for more than 3 years was done. Differences in the changes in standard deviation scores for height were measured and grouped according to the karyotype and age at the start of treatment.

Results: The response to recombinant growth hormone was an increase in the height of the subjects to a mean value of $0.58 \mathrm{SD}$ above the mean for normal population at the end of the 3-year treatment. The increment in both the height and its standard deviation score was greatest in subjects who started the treatment before 8 years of age. The height increment was highest in the first year when the treatment was started before 12 years of age. The age at commencement of treatment was inversely associated with the standard deviation score of the height at the third year. The patient's karyotype did not account for the difference in height gain.

Conclusions: Early growth hormone administration in subjects with Turner syndrome improves height response to the treatment.

\section{EPO-271 Turner Syndrome \\ Thyroid diseases in 55 Chinese girls with Turner syndrome}

Ruimin Chen; Ying Zhang; Xiaohong Yang; Xiangquan Lin Fuzhou Children's Hospital of Fujian, Endocrinology, Fuzhou, China

Background: Turner Syndrome (TS) is characteristic with reduced adult height and gonadal dysgenesis, associated with a number of complications including thyroid diseases.

Objective and hypotheses: The purpose of this study was to investigate the frequency of thyroid diseases and the association between thyroid autoantibodies (TAA) and thyroid dysfunction, age, and karyotypes.

Methods: Fifty-five girls with TS were diagnosed by chromosome analyses and were divided into 2 groups according to whether there was TAA-positive or not: TAA-positive group and TAA-negative group. Thyroid autoantibodies (antithyroglobulin antibody, thyroperoxidase antibody), thyroid function (free T3, free T4 and TSH) were determined with immunochemiluminescent. Ultrasound was detected in TAA-positive group.

Results: Thyroid functions in 34 girls with TAA-negative were normal. Of the 21 girls with TAA-positive $(21 / 55,38.2 \%), 7$ girls were hypothyroidism and 3 girls were hyperthyroidism. All of 21 girls with TAA-positive were diagnosed as Hashimoto's thyroiditis. As compared with the girls with TAAnegative $(9.95 \pm 4.50)$, the age of girls with TAA-positive group (12.16 \pm 2.55$)$ were significantly higher $(\mathrm{P}<0.05)$. There was no TAA-positive case under 5 years old, $31.3 \%(5 / 16)$ TAA-positive in 5 10years old, $48.1 \%(13 / 27)$ in $10 \sim 15$ years old and $50.0 \%(3 / 6)$ above the age of 15 . There were no significant differences in the numbers of TAA-positive among different karyotypes $(\mathrm{P}>0.05)$. Thyroid ultrasound in girls with positive-TAA showed the abnormal echogenicity in bilateral lobus lateralis of thyroid.

Conclusions: TS past the age of 5 years are trend to cause Hashimoto's thyroiditis which lead to thyroid dysfunction. We suggest that thyroid function should be evaluated yearly in girls with TS after 5 years old. There is not a specific association between the incidence of autoimmune thyroid disease and certain karyotypes.

\section{EPO-272 Turner Syndrome \\ Turner syndrome in last years}

Isabel Rios Orbañanos ${ }^{1}$; Rebeca Sanchez Sanchez'; Gema Grau Bolado'; Amaya Rodriguez Estevez';

Itxaso Rica Etxebarria ${ }^{1}$; Amaya Vela Desojo ${ }^{1}$

${ }^{1}$ Hospital Universitario de Cruces, Paediatric Endocrinology, Barakaldo, Spain; ${ }^{2 H}$ ospital Universitario de Cruces, Endocrinology, Barakaldo, Spain

Background: Turner syndrome is characterized by a great variability of clinical manifestations caused by total or partial loss of X-chromosome.

Objective and hypotheses: To know the situation in adulthood of a group of patients with Turner syndrome that were diagnosed in our Hospital and to describe the features at diagnosis.

Methods: Retrospective review of Turner syndrome patients diagnosed in our section using medical records supplemented by telephone survey.

Results: 46 female were studied, with mean actual age of 22.95 years (range $2-38$ ) and 4.71 at diagnosis. $71.7 \%$ showed mosaicism. In the 32 adult patients, follow-up is carried out by Endocrine (37.5\%), Gynecologist (34.4\%) and in Primary Care (12.5\%). 68.75\% have pathological short stature, regardless of treatment. Densitometry was performed only in 6 cases, presenting one case osteopenia and another osteoporosis. $25 \%$ have dyslipidemia, $21.9 \%$ hypertension, a case shows glucidic profile alteration and two diabetes mellitus. $65.6 \%$ had no spontaneous puberal development, receiving all but one, replacement therapy. Three patients have sons with ovule donation. Two have died. $21.7 \%$ need assistance during school and the level of studies reached in adulthood is low $15.6 \%$, medium $18.8 \%$ and high $40.6 \%$. Two cases present psychiatric pathology. Globally, short stature is the most common cause of diagnosis $(54.3 \%)$ with increased importance of prenatal diagnosis in most recent cases $(36 \%$ versus $3 \%$ in $\geq 16$ years). In terms of clinical manifestations: repeated middle ear infection appears in 39.1\%; autoimmune disease in $15.2 \%$, especially thyroiditis $(8.7 \%) ; 13 \%$ of cases present horseshoe kidney, $13 \%$ have developed eye problems and $6.5 \%$ aortic pathology. $71.7 \%$ have been treated with growth hormone, associated with oxandrolone in $26.1 \%$. Conclusions: Short stature is the most common cause of diagnostic in Turner syndrome, detecting in most of them genetic mosaicism. Most usual clinical manifestations are repeated otitis media, short stature and gonadal failure. $60 \%$ achieve medium-high level studies. 
EPO-273 Turner Syndrome

Efficacy and safety of use of the domestic somatropin "Rastan" in girls with Turner's syndrome

Nataly Volevodz'; Oleg Malievsky²; Valentina Peterkova ${ }^{1}$

${ }^{1}$ Endocrinology Research Centre, Institute of Paediatric Endocrinology,

Moscow, Russian Federation; ${ }^{2}$ Bashkir State Medical University,

Department of Paediatrics, Ufa, Russian Federation

Background: The low height is observed in $95 \%$ of girls with Turner's syndrome. Their mean final height does not exceed $140-147 \mathrm{~cm}$. Use of somatropin allows increasing the rate of growth in children with Turner's syndrome and improving the growth prognosis.

Objective and hypotheses: The objective of the present study was to evaluate efficacy and safety of treatment of girls with Turner's syndrome by recombinant growth hormone Rastan («Farmstandart-UfaVITA», Ufa, Russia). Population and/or methods: The total of 29 girls under this condition were available for observation. Their chronological age at the time of diagnosis was, on average, 7.971.2 years at the time of diagnosis and 9.871.6 at the onset of therapy; the bone age was 7.571.1 years. Duration of the treatment was 24 months. Recombinant somatropin was used in the form of lyophilizate $(1.33 \mathrm{mg})$ to prepare solution for subcutaneous injections at a dose of 0.05 $\mathrm{mg} / \mathrm{kg}$ of body weight. The solution was administered daily at evening hours. Results: The mean growth rate of the patients with Turner's syndrome was estimated to be $4.2+0.6 \mathrm{~cm} /$ year prior to Rastan therapy, $8.7+0.6 \mathrm{~cm} /$ year during the first 12 months of the treatment, and $6.1+1.2 \mathrm{~cm} / \mathrm{yr}$ in the second year. Overall, the height of the girls increased by $0.84 \mathrm{SD}$ within the two years. No adverse effects of the therapy were documented.

Precise conclusions: The use of recombinant somatropin Rastan is an efficacious and safe therapeutic modality for acceleration of growth in girls with Turner's syndrome.

\section{EPO-274 Turner Syndrome}

\section{Normal male phenotype in a 45 X SRY + boy with short stature and mild Turner stigmata} Asteroula Papathanasiou'; Georgia Ntali'; Christalena Sofocleous ${ }^{2}$; Kyriaki Karavanaki ; Efthymia Tsiapali'; Georgia Tsangaraki'; Helen Fryssira²

1"P\&A. Kyriakou" Children's Hospital, Pediatric Endocrinology, Athens, Greece; 'University of Athens, Medical Genetics, Athens, Greece;

${ }^{3}$ University of Athens, Second Pediatric Department, Athens, Greece

Background: Most patients with 45 X0 karyotype are phenotypically females with Turner syndrome(TS). Male patients with Turner stigmata and non-mosaic deletions of $\mathrm{Y}$ are rarely reported and interesting regarding genetic mechanisms, fertility issues and future risk of malignancy.

Objective and hypotheses: To report the case of a boy with mild dysmorphic features with a karyotype $45 \mathrm{XO}, \mathrm{SRY}+$. We hypothesize that male phenotype is due to SRY translocation to another chromosome during paternal meiosis and subsequent non-disjunction and loss of maternal $\mathrm{X}$.

Case report: A 4-year old boy was referred for evaluation of growth delay. Clinical examination revealed mild dysmorphic features reminiscent of TS. External genitalia were normal with scrotal located testes. No Müllerian structures were identified during laparoscopy. The patient was treated successfully with rhGH and entered puberty spontaneously. At the age of 13 gonadal biopsy was performed and histology revealed immature tubular structures without signs of carcinoma in situ (negative markers c-kit, oct 3). The presence of SRY explains normal male gonadal development but lack of Yq including azoospermia factor will probably cause infertility.

Results: The karyotype of several tissues (peripheral blood, fibroblasts from scrotal skin, testis) was 45 ,XO.FISH was compatible with complete absence of chromosome Y.PCR using Y specific primers disclosed the presence of an intact SRY on the Yp despite absence of other Y chromosome sequences. Haplotype analysis indicated that the $\mathrm{X}$ chromosome was of paternal origin. SHOX haploisufficiency was documented.

Conclusions: Physicians should have a high index of suspicion for dysmorphic features suggestive of TS even in short boys. Karyotyping and molecular analysis should be performed to diagnose possible chromosomal defects associated with gonadal dysgenesis. The correct diagnosis enables early initiation of growth promoting therapy, treatment of possible associated diseases and follow up of the dysgenetic testes for signs of malignancy.

\section{EPO-275 Turner Syndrome}

Metabolic and anthropometric parameters are related to carotid intima thickness in Turner syndrome

Nesibe Akyürek; Mehmet Emre Atabek; Sevil Ari Yuca;

Beray Selver Eklioglu; Hayrullah Alp

Konya University School of Medicine, Div of Pediatric Endocrinology, Konya, Turkey

Background: There is an increased risk of cardiovascular morbidity in women with Turner syndrome (TS), presumably due to their risk of cardiovascular malformations, renal abnormalities, and hypertension. We evaluated common carotid artery intima-media thickness (CCA-IMT), a marker of early carotid atherosclerosis, and investigated their relation to metabolic and anthropometric parameters in children and adolescents with TS.

Methods: 25 patients with TS and 25 healthy controls were included in the study. CCA-IMT was measured by high resolution B-mode ultrasonography. Metabolic and anthropometric parameters such as serum lipids levels, insulin resistance ,body mass index, waist-hip ratio and blood pressure were also evaluated.

Results: Mean age, serum lipid levels, insulin resistance indexes, body mass index, waist-hip ratio, blood pressure and CCA-IMT values were similar in the two groups. In the TS group, CCA-IMT correlated positively with systolic blood pressure $(r=0.54, \mathrm{p}=0.031)$ and negatively with $\mathrm{FGIR}(\mathrm{r}=-0,525$ $\mathrm{p}=0.045$ ).

Conclusions: The relationship between CCA-IMT, systolic blood pressure and FGIR may indicate the impact of early events in the genesis of atheroma in TS These findings may have an important role in increasing cardiovascular risk in these patients.

\section{EPO-276 Turner Syndrome}

Who, when, what to tell when gonadectomy is the case? An integrated diagnostic process for gonadectomy in Turner syndrome

\section{Maura Foresti'; Benedetta Vestrucci'; Laura Nardi';}

Maria Teresa Assante'; Emanuela Scarano²; Annamaria Perri2;

Federica Tamburrino ${ }^{2}$; Laura Mazzanti

${ }^{1}$ Clinical Psycology Service, Department of Pediatrics, S.Orsola-

Malpighi Hospital, University of Bologna, Bologna, Italy; ${ }^{2}$ Rare Disease

and Auxology Unit, Department of Pediatrics, S.Orsola-Malpighi

Hospital, University of Bologna, Bologna, Italy

Background: We will describe the cases of two girls who were diagnosed with Turner syndrome and were prescribed gonadectomy at a prepuberal age. Objective and hypotheses: At first the diagnosis was communicate to the patients' parents, psychological aid was offered to them. The purpose of these sessions was to help them in the decision of what to tell their daughters and how to tell them. To begin with, in both cases the parents were confused and worried and they thought it was better not to tell the truth.

Results: The psychological support helped them to contain their grief: they started thinking their daughters could be happy even without ovaries and that by lying they could also lose their daughters' trust. In both cases the parents decided to tell the truth. They also accepted some extra help from the doctors in communicating the medical aspects of the diagnosis to the girls. In one case the parents had never talked about sex issues with their daughter, so they needed to be helped. The parents agreed to encourage the girls to see a psychologist and the girls accepted. Both the girls had imagined that they could be at death's door and were relieved to learn the truth. However, the diagnosis, especially the one about infertility, made them suffer a great deal The grief seemed to become bearable with the comfort offered by the parents and through talking to the psychologist and the doctors. A year later the parents reported that their daughters were talking freely about their condition; that the girls thought it was good for them to be properly informed and that they trusted their parents and the doctors.

Conclusions: This experience seems to be a good example of the importance of giving accurate support to the patient and her family in cases of gonadectomy. 


\section{EP0-277 Turner Syndrome \\ Down-Turner syndrome: a patient with a particular phenotype \\ Silvia Bergamaschi'; Claudia Giavoli'; Silvana Guerneri; Emanuele Ferrante ${ }^{1}$; Renato Fortuna ${ }^{2}$; Donatella Milani; \\ Eriselda Profka ${ }^{1}$; Francesca Menni ${ }^{4}$; Marta Cerutti ${ }^{5}$; \\ Gianantonio Manzoni6; Alfredo Berrettini6; Santiago Vallasciani ${ }^{6}$; Anna Spada'; Paolo Beck-Peccoz ${ }^{1}$ \\ 'Fondazione IRCCS Ca' Granda, Ospedale Maggiore Policlinico, University of Milan, Department of Medical Sciences, Endocrinology and Diabetology Unit, Milan, Italy; '²Fondazione IRCCS Ca' Granda, Ospedale Maggiore Policlinico, Department of Maternal and Pediatric Sciences, Medical Genetics Lab, Milan, Italy; ${ }^{3}$ Fondazione IRCCS Ca' Granda, Ospedale Maggiore Policlinico, Medical Genetics Unit, Milan, Italy; 'Fondazione IRCCS Ca' Granda, Ospedale Maggiore Policlinico, Pediatric Unit, Milan, Italy; ${ }^{5}$ Fondazione IRCCS Ca' Granda, Ospedale Maggiore Policlinico, University of Milan, Pediatric Unit, Milan, Italy; ${ }^{6}$ Fondazione IRCCS Ca' Granda, Ospedale Maggiore Policlinico, \\ Pediatric Urology Unit, Milan, Italy}

Introduction: Double aneuploidy involving both sex and autosomal chromosomes (chr) is very rare, Down-Turner syndrome being the most frequent. Fourty-seven cases of Down-Turner mosaicism have been reported, only 9 with a kariotype containing $\mathrm{Y}$ chr (phenotype: 7 male, 2 ambiguous genitals). Case report: We describe a girl affected with Down-Turner syndrome. The genetical analysis on peripheral lymphocytes, performed 2 months after birth because of mild facial dysmorphisms, showed a kariotype $45, \mathrm{X} / 47, \mathrm{XY},+21$ $(84 / 16 \%)$.

Analysis of cultured skin fibroblasts confirmed the kariotype, with similar proportion of mosaicism $(79 / 21 \%)$.

The girl came to our attention at the age of 15.4 yrs. She presented few, if any characteristics of Down syndrome apart from primary autoimmune hypothyroidism. The phenotype of Turner syndrome was mild too: wide thorax with a large internipple distance, short neck, no signs of puberty with female external genitals, no mental retardation. Hormonal assessment showed hypergonadotropic hypogonadism (FSH 93.2 U/L, LH $37.7 \mathrm{U} / \mathrm{L}$, estradiol $5 \mathrm{pg} / \mathrm{mL}$, AMH $<0.1 \mathrm{ng} / \mathrm{mL}$, testosterone $0.24 \mathrm{ng} / \mathrm{mL}$ ).

MRI abdominal imaging showed streak dysgenetic gonads with an infantile uterus. Because of the presence of $Y \mathrm{chr}$, along with streak non-functioning gonads, a prophylactic gonadectomy was performed and estrogenic replacement was started. Histological examination revealed a mixed gonadal dysgenesis, with the presence of both ovarian and testis tissue.

Cytogenetic analysis performed with conventional techniques confirmed the kariotype 45,X/47,XY,+21 (77/23\%) in gonadal fibroblasts. FISH analysis with SRY probe showed the presence of a hybridization signal on the $Y \mathrm{chr}$. Conclusions: We report the first case of Down-Turner syndrome presenting a $\mathrm{Y}$ chr with a female phenotype. To confirm the mosaicism as causative event, ongoing genetic studies will be carried out to exclude the presence of chimerism.

\section{EPO-278 Turner Syndrome \\ Bone age (BA) influence at the beginning of the treatment with growth hormone (GH) in Turner's syndrome (TS): comparison with a control group without treatment \\ Lilian H Solitari; Letícia Barberi; Márica N Melo; Sílvia C Zagui; \\ Bruno H Ranciaro; Rosamaria G Raza; Magali Zampieri; \\ Maria Helena Senger; João C Ramos-Dias; Alcinda Aranha Nigri \\ Faculdade de Ciências Médicas e da Saúde de Sorocaba - FCMS - \\ PUC/SP, Medicine, Sorocaba, Brazil}

Background: Short stature is a common characteristic in girls with Turner's Syndrome (TS).

Objective and hypotheses: The aim of our study was to evaluate the result of treatment with GH in the final height of TS patients.

Methods: This was a retrospective study of $34 \mathrm{TS}$ patients, categorized into two groups, control group (CG) $n=10$ and treated group (TG) $n=24$. This group was sub-categorized in $T G$ with $B A \leq 10$ years $(n=8)$ and $T G$ with $\mathrm{BA}>10$ years $(\mathrm{n}=16)$.

Results: The karyotype $45, \mathrm{X}$ was observed in $41,2 \%$ and median of years at the diagnostic was $10,1 \pm 3,1$ years. In the TG $(n=24)$ the mean height in the end of treatment was $147,5 \pm 7,3 \mathrm{~cm}$ and in the CG $(\mathrm{n}=10)$ was $143,2 \pm 6,6 \mathrm{~cm}$
$(\mathrm{p}=\mathrm{NS})$. In the TG BA $\leq 10 \mathrm{years}(\mathrm{n}=8)$ the final height was $151,3 \pm 5,4 \mathrm{~cm}$ and in GT, BA>10years $(\mathrm{n}=16) 145,6 \pm 7,6 \mathrm{~cm}$.

The TG $\mathrm{BA} \leq 10$ years presented advantage when compared to the $\mathrm{CG}(\mathrm{p}<0,01)$ as well as the TG BA $>10$ years $(p<0,05)$ in terms of final stature

Conclusions: The TG presented the best average height in the end of treatment when the GH was started with $\mathrm{BA} \leq 10$ years, showing the importance of a precocious diagnosis and beginning of treatment in order to minimize the adult short stature in girls with TS. 